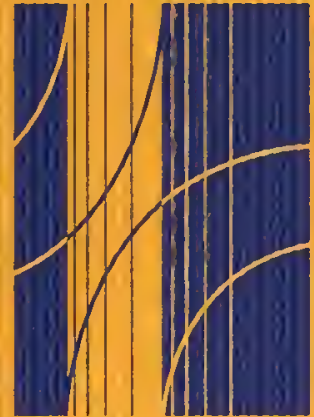

Joint

Transportation

Research

Program

J T R P

FHWA/IN/JTRP-98/17

Final Report

PERFORMANCE OF BRIDGE DECKS AND GIRDERS WITH LIGHTWEIGHT AGGREGATE CONCRETE

Volume 1 of 2

Julio Ramirez

Jan Olek

Eric Rolle

Brian Malone

October 2000

Indiana

Department

of Transportation

Purdue

University 

Final Report

FHWA/IN/JTRP-98/17

PERFORMANCE OF BRIDGE DECKS AND GIRDERS WITH

LIGHTWEIGHT AGGREATE CONCRETE

(Volume 1 of 2)

\author{
Julio A. Ramirez \\ and \\ Jan Olek \\ Principal Investigators \\ and \\ Eric Rolle \\ and \\ Brian Malone \\ Research Assistants \\ School of Civil Engineering \\ Purdue University
}

Joint Highway Research Program

Project Number: C-36-56MM

File Number: 7-4-39

Conducted in Cooperation with the Indiana Department of Transportation and the

Federal Highway Administration

The contents of this report reflect the views of the authors, who are responsible for the facts and the accuracy of the data presented herein. The contents do not necessarily reflect the views or policies of the Federal Highway Administration and the Indiana Department of Transportation. This report does not constitute a standard, specification, or regulation.

Purdue University

West Lafayette, IN 47907

October 2000 


\begin{tabular}{l|l}
\hline 1. Report No. & 2. Government Accession No. \\
FHWA/IN/JTRP-98/17 & \\
\hline
\end{tabular}

4. Title and Subtille

Performance of Bridge Decks and Girders with Lightweight Aggregate Concrete (2 Volumes)
3. Recipienl's Catalog No.

5. Report Date

October 2000

6. Performing Organization Code

8. Performing Organization Report No.

FHWA/N/JTRP-98/17

10. Work Unit No.

9. Performing Organization Name and Address

Joint Transportation Research Program

1284 Civil Engineering Building

Purdue University

West Lafayette, Indiana 47907-1284

11. Contract or Grant No.

SPR-2131

12. Sponsoring Agency Name and Address

Indiana Department of Transportation

State Office Building

100 North Senate Avenue

lndianapolis, IN 46204
13. Type of Report and Period Covered

Final Report

14. Sponsoring Agency Code

\section{Supptementary Notes}

Prepared in cooperation with the Indiana Department of Transportation and Federal Highway Administration.

\section{Abstract}

Structural lightweight concrete is a very versatile material and Haydite and Minergy lightweight aggregates can be utilized in the mixture development of concrete for use in girders and decks in bridges. More widespread use of lightweight aggregates (LWA) would result in savings in construction cost due to considerable dead load reduction.

The materials phase of this research study evaluated the fresh concrete properties (slump, unit weight, and air coritent), the mechanical properties of hardened concrete (compressive strength, tensile strength, flexural strength, static and dynamic modulus of elasticity, Poisson's Ratio, and temperature development), and durability related parameters (air void distribution, freeze-thaw resistance, chloride permeability, resistance to scaling, and drying shrinkage). In addition, selected properties (24-hour. absorption, bulk specific gravity, size distribution, and intemal pore structure) of the lightweight aggregates were also determined.

Lightweight aggregate mixes used in this study were proportioned using water-cement ratio/strength method in order to better address the issue of producing mixes that would satisfy both durability and strength requirements. Target compressive strengths for bridge girder concrete were 42 and $69 \mathrm{MPa}$ (6000 and 10,000 psi), and $31 \mathrm{MPa}(4500 \mathrm{psi})$ in the case of bridge deck concrete.

In the structural phase of the study the shear strength of reinforced and prestressed concrete lightweight concrete (LWC) beams was evaluated. In the Kettelhut Structural Engineering Laboratory twelve reinforced and four prestressed concrete beams were tested to failure. The experimental shear capacities were compared to calculated shear strength values from the 1995 American Concrete Institute Building Code and the 1994 AASHTO LRFD Standard Specifications.

Based on the test results from this study it is recommended that water-cementitious ratio be used for proportioning of lightweight concrete mixes and that trial batches be prepared and tested before actual field application. To ensure adequate durability, lightweight aggregate concrete should be allowed to dry before exposing it to cycles of freezing and thawing. On the structural side, both the ACI and the AASHTO LRFD Specifications were found to yield conservative estimates of the shear strength of LWC reinforced and prestressed beams provided adequate detailing of the reinforcement is provided. More work is needed regarding the minimum amount of shear reinforcement for high strength lightweight concrete beams.

\section{Key Words}

concrete, lightweight aggregate, shear, strength, durability, freeze-thaw, mix proportioning, properties bridges, beams, reinforced, prestressed.

\section{Distribution Statement}

No restrictions. This document is available to the public through the National Technical Information Service, Springfield, VA 22161
19. Security Classif. (of this report) Unclassified
20. Security Classif. (of this page) Unclassified

\begin{tabular}{c|c}
$\begin{array}{c}\text { 21. No. of Pages } \\
616(2 \text { volumes })\end{array}$ & 22. Price \\
\hline
\end{tabular}




\section{Implementation Report}

Compared to the ordinary (normal weight) concrete lightweight aggregate structural concrete offers several technical advantages including high strength to unit weight ratio and excellent durability. Recent advances in the formulation and use of mineral and chemical admixtures in production of concrete made it possible to attain lightweight aggregate concrete with a very high strength while keeping the density low. These advancements open interesting and promising possibilities for the use of this material in reinforced and prestressed concrete structures.

Pursuant to findings presented in this report the following suggestions are being provided as a means of implementing lightweight aggregate concrete in the state of Indiana

\section{- Development of full-scale field project}

During the last few years INDOT successfully used semi-lightweight concrete in production of girders for some of the new bridges. The findings of this report fully warrant initiation of a coordinated effort to extent the use of lightweight aggregate concrete to bridge decks. The lightweight concrete mixes designed and produced as a part of this study can be used as a starting point for development of field mixes that can be used for construction of all-lightweight concrete bridge.

\section{- Development of provisional specifications}

In order to accommodate the use of lightweight concrete in actual bridge construction a set of provisional specifications should be developed utilizing the key findings of this report which are briefly summarized below:

With respect to estimating the shear capacity of lightweight aggregate concrete beams it is recommended that the ACI 318-95/AASHTO $16^{\text {th }}$ Edition procedure be used rather than the AASHTO LRFD procedure. Also, the amount of minimum shear reinforcement needs to be increased, as lightweight concrete will have lower postcracking reserve strength.

In proportioning the lightweight aggregate concrete the use of saturated aggregate and control of water-cement ratio offered the most consistent results. Before the production of the actual field mixes trial mixes will always be necessary to fix the total amount of mixing water to ensure desired workability and strength. In the mixing plant the moisture content of each production batch of lightweight aggregate needs to be determined and any variations from the target water content need to be properly compensated for. For lightweight concrete intended for the bridge deck the water-cemetitious material ratio higher than 0.42 is not recommended. 
Digitized by the Internet Archive in 2011 with funding from

LYRASIS members and Sloan Foundation; Indiana Department of Transportation 


\section{TABLE OF CONTENTS}

LIST OF TABLES viii

LIST OF FIGURES xvi

NOTATION $x l v$

CHAPTER 1: INTRODUCTION 1

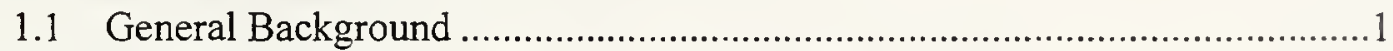

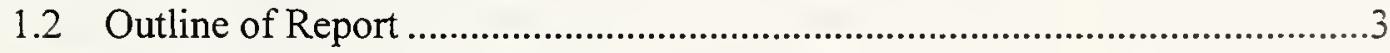

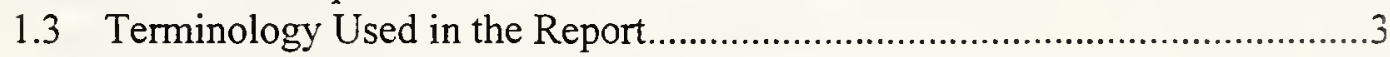

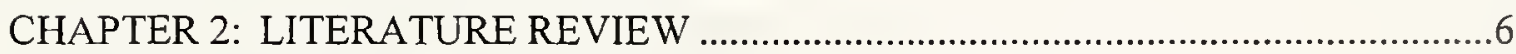

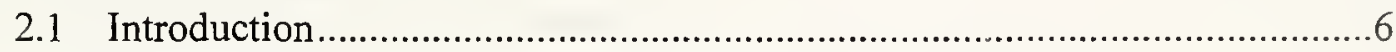

2.2 Characteristics of Lightweight Aggregates..................................................

2.3 Structural Lightweight Concrete in Bridge Construction ............................... 9

2.4 Physical and Mechanical Properties of Lightweight Concrete .....................15

2.5 Durability of Lightweight Concrete...........................................................19

2.6 Mix Design of Structural Lightweight Concrete ..........................................20

2.7 Shear Strength of Concrete Beams .........................................................24

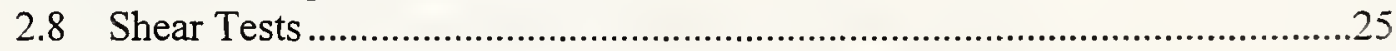

2.9 Methods of Calculating Shear Strength ....................................................28

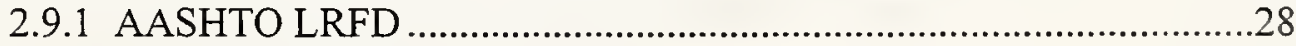

2.9.1.1 Simplified Procedure ..............................................................29

2.9.1.2 General Procedure.................................................................30

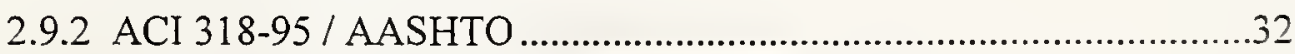

2.9.2.1 Reinforced Concrete ................................................................33

2.9.2.2 Prestressed Concrete ..................................................................34

CHAPTER 3: PROBLEM STATEMENT AND RESEARCH OBJECTIVES ..................41

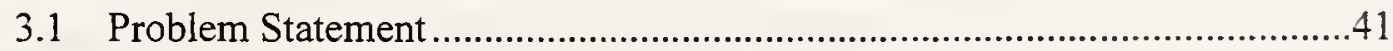

3.2 Research Objectives...................................................................................43

3.3 Scope of the Research.............................................................................. 


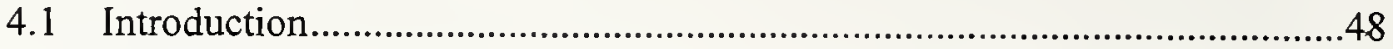

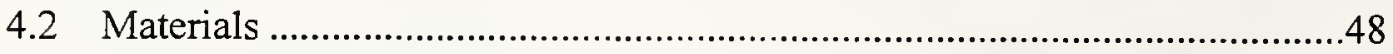

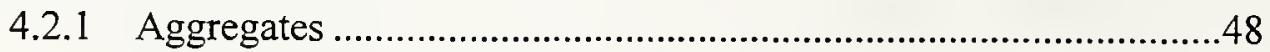

4.2.1.1 Haydite Lightweight Aggregate.......................................49

4.2.1.2 Minergy Lightweight Aggregate.....................................51

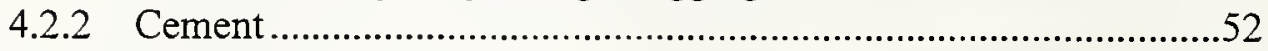

4.2.3 Silica Fume and Fly Ash..........................................................52

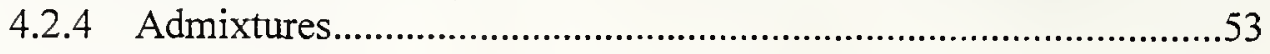

4.3 Mixture Proportioning .........................................................................53

4.3.1 Pretreatment Procedures for Lightweight Aggregate .....................53

4.3.2 Development of Mixture Proportions ..............................................55

4.3.2.1 Mixture Proportioning of Concrete for Girders .........56

4.3.2.2 Mixture Proportioning of Concrete for Decks ...........57

4.3.3 Practical Issues Related to Field Mixture Production....................58

4.3.3.1 Procedure Used for Batching Lightweight Concrete in the Field ....................................................................59

4.3.3.2 Method of Material Proportioning in the Field.........59

4.3.3.3 Difficulty in Plant Production of Lightweight

Concrete

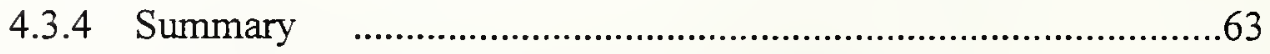

4.4 Concrete for Beam Specimens..............................................................63

4.4.1 Concrete for Reinforced Concrete Specimens ...............................64

4.4.2 Concrete for Prestressed Concrete Specimens.................................65

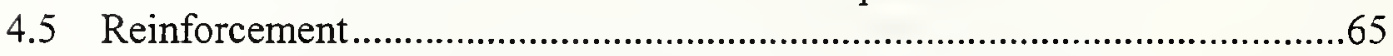

4.5.1 Reinforcement for Reinforced Concrete Specimens.......................66

4.5.2 Reinforcement for Prestressed Concrete Specimens ......................66

4.6 Description of Specimens ..........................................................................67

4.6.1 Reinforced Concrete Specimens .................................................67

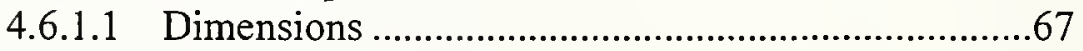

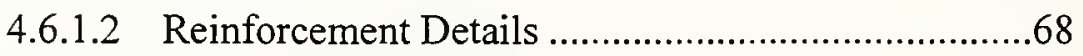

4.6.1.3 Casting and Curing ...................................................68

4.6.2 Prestressed Concrete Specimens ....................................................69

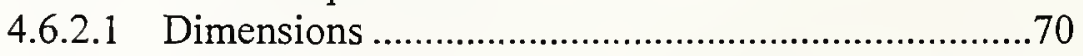

4.6.2.2 Reinforcement Details ............................................70

4.6.2.3 Casting and Curing ....................................................70

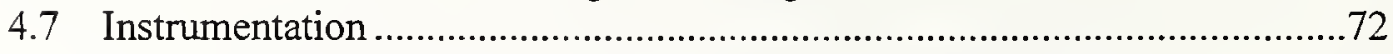

4.7.1 Instrumentation of Reinforced Concrete Specimens ......................72

4.7.2 Instrumentation for Prestressed Concrete Specimens......................74

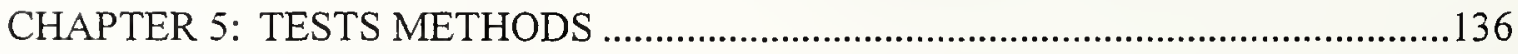

5.1 Testing of Fresh Concrete Properties ...................................................136

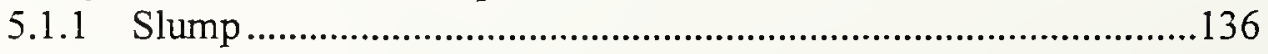




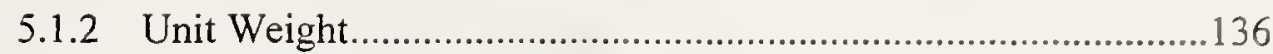

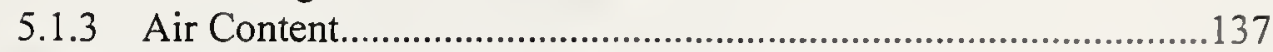

5.2 Testing of Hardened Concrete ...................................................................137

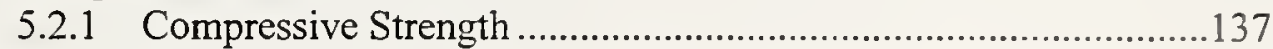

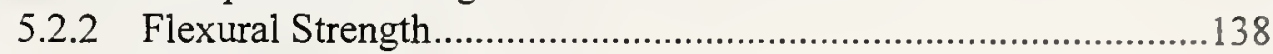

5.2.3 Split Tensile Strength..................................................................138

5.2.4 Static and Dynamic Modulus of Elasticity, Poisson' Ratio .........139

5.2.5 Density of Hardened Concrete.....................................................140

5.2.6 Temperature Development.........................................................140

5.3 Testing of Durability Properties of Concrete..............................................141

5.3.1 Air Void Distribution..............................................................141

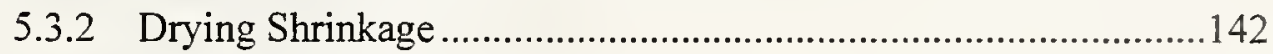

5.3.3 Freeze-Thaw Resistance .......................................................142

5.3.4 Rapid Chloride Ion Permeability ..............................................144

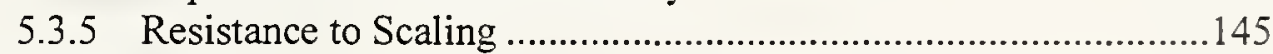

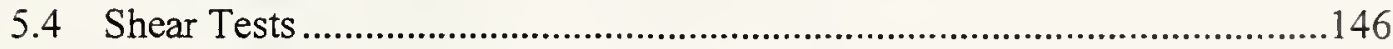

5.4.1 Reinforced Concrete Specimens ................................................146

5.4.1.1 Loading Frame ............................................................146

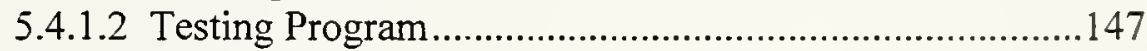

5.4.2 Prestressed Concrete Specimens..................................................147

5.4.2.1 Loading Frame ..............................................................147

5.4.2.2 Testing Program............................................................148

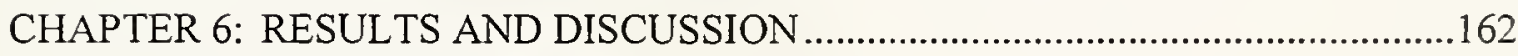

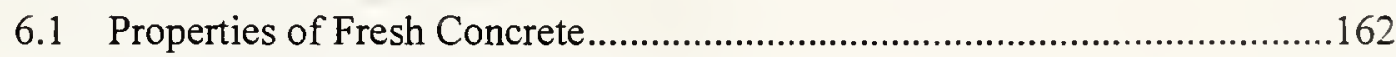

6.2 Properties of Hardened Concrete .............................................................163

6.2.1 Compressive Strength ............................................................163

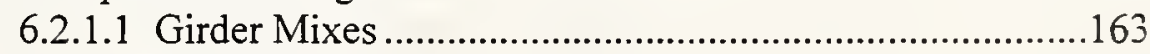

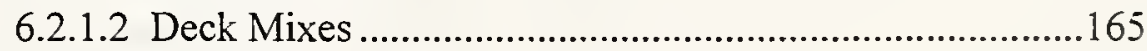

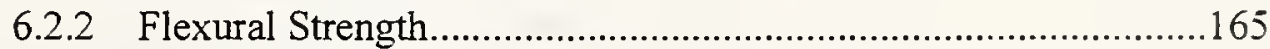

6.2.3 Split Tensile Strength.................................................................166

6.2.4 Static and Dynamic Modulus of Elasticity, and Poisson's Ratio.167

6.2.5 Density of Hardened Concrete......................................................168

6.2.6 Temperature Development........................................................168

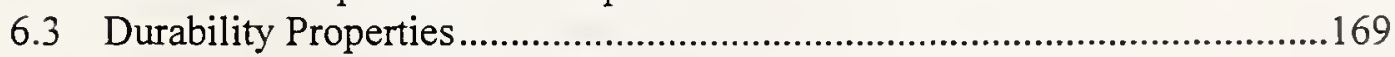

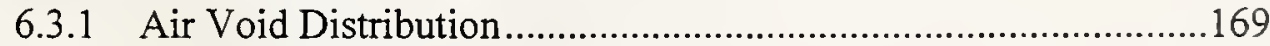

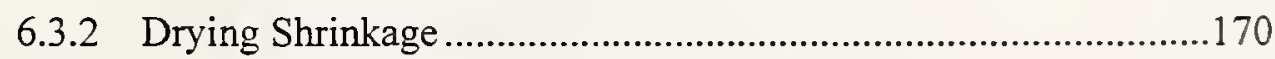

6.3.3 Freeze-Thaw Resistance .......................................................171

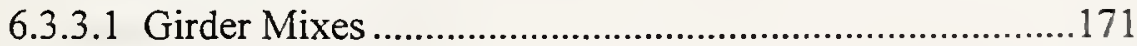

6.3.3.2 Deck Mixes .................................................................172

6.3.4 Rapid Chloride Ion Permeability ............................................174

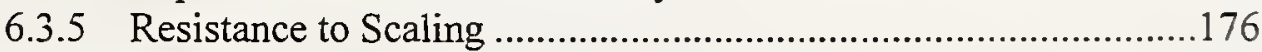

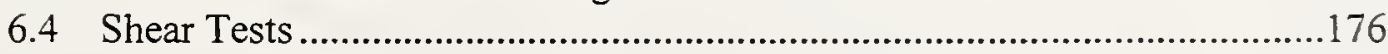

6.4.1 Reinforced Concrete Specimens ...............................................178 
6.4.1.1 Specimen 1-NWLA 180

6.4.1.1.1 Introduction ........................................18

6.4.1.1.2 Deflections.............................................181

6.4.1.1.3 Longitudinal Mild Steel Strains ..............181

6.4.1.1.4 Stirrup Strains........................................181

6.4.1.1.5 Concrete Surface Strains ........................182

6.4.1.1.6 Failure Observations..............................182

6.4.1.2 Specimen 2-NWLB.................................................183

6.4.1.2.1 Introduction .........................................183

6.4.1.2.2 Deflections..............................................183

6.4.1.2.3 Longitudinal Mild Steel Strains ..............183

6.4.1.2.4 Stirrup Strains..........................................184

6.4.1.2.5 Concrete Surface Strains ..........................184

6.4.1.2.6 Failure Observations...............................184

6.4.1.3 Specimen 3-NWLC.....................................................185

6.4.1.3.1 Introduction ........................................185

6.4.1.3.2 Deflections.............................................185

6.4.1.3.3 Longitudinal Mild Steel Strains ..............185

6.4.1.3.4 Stirrup Strains ..........................................186

6.4.1.3.5 Concrete Surface Strains .........................186

6.4.1.3.6 Failure Observations...............................186

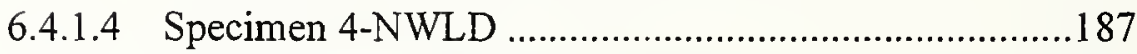

6.4.1.4.1 Introduction ............................................187

6.4.1.4.2 Deflections.............................................187

6.4.1.4.3 Longitudinal Mild Steel Strains ..............188

6.4.1.4.4 Stirrup Strains .........................................188

6.4.1.4.5 Concrete Surface Strains ..........................188

6.4.1.4.6 Failure Observations................................188

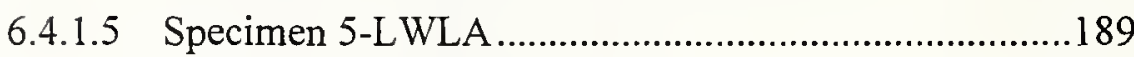

6.4.1.5.1 Introduction ...........................................189

6.4.1.5.2 Deflections.............................................189

6.4.1.5.3 Longitudinal Mild Steel Strains ..............190

6.4.1.5.4 Stirrup Strains ..........................................190

6.4.1.5.5 Concrete Surface Strains ..........................190

6.4.1.5.6 Failure Observations.................................191

6.4.1.6 Specimen 6-LWLB ....................................................191

6.4.1.6.1 Introduction .............................................191

6.4.1.6.2 Deflections................................................191

6.4.1.6.3 Longitudinal Mild Steel Strains ..............192

6.4.1.6.4 Stirrup Strains ...........................................192

6.4.1.6.5 Concrete Surface Strains .........................192

6.4.1.6.6 Failure Observations................................193

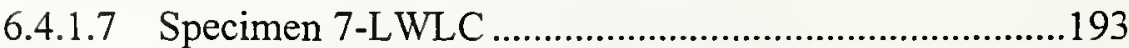

6.4.1.7.1 Introduction ..........................................193

6.4.1.7.2 Deflections.............................................193 
6.4.1.7.3 Longitudinal Mild Steel Strains .............194

6.4.1.7.4 Stirrup Strains........................................194

6.4.1.7.5 Concrete Surface Strains .........................194

6.4.1.7.6 Failure Observations..............................195

6.4.1.8 Specimen 8-LWLD ......................................................195

6.4.1.8.1 Introduction ..........................................195

6.4.1.8.2 Deflections..............................................195

6.4.1.8.3 Longitudinal Mild Steel Strains .............196

6.4.1.8.4 Stirrup Strains..........................................196

6.4.1.8.5 Concrete Surface Strains ........................196

6.4.1.8.6 Failure Observations..............................197

6.4.1.9 Specimen 9-NWLD ...............................................197

6.4.1.9.1 Introduction ..........................................197

6.4.1.9.2 Deflections...............................................197

6.4.1.9.3 Longitudinal Mild Steel Strains ..............198

6.4.1.9.4 Stirrup Strains.......................................198

6.4.1.9.5 Concrete Surface Strains ........................198

6.4.1.9.6 Failure Observations................................198

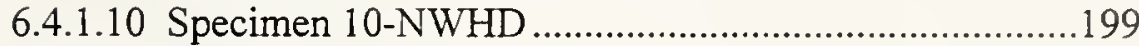

6.4.1.10.1 Introduction ............................................199

6.4.1.10.2 Deflections.............................................199

6.4.1.10.3 Longitudinal Mild Steel Strains .............200

6.4.1.10.4 Stirrup Strains........................................200

6.4.1.10.5 Concrete Surface Strains ........................200

6.4.1.10.6 Failure Observations.............................201

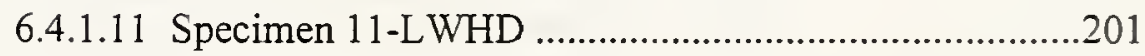

6.4.1.11.1 Introduction ..........................................201

6.4.1.11.2 Deflections..............................................201

6.4.1.11.3 Longitudinal Mild Steel Strains .............202

6.4.1.11.4 Stirrup Strains........................................202

6.4.1.11.5 Concrete Surface Strains ........................202

6.4.1.11.6 Failure Observations..............................203

6.4.1.12 Specimen 12-NWHD ...................................................203

6.4.1.12.1 Introduction ..............................................203

6.4.1.12.2 Deflections..............................................203

6.4.1.12.3 Longitudinal Mild Steel Strains ..............204

6.4.1.12.4 Stirrup Strains.........................................204

6.4.1.12.5 Concrete Surface Strains ........................204

6.4.1.12.6 Failure Observations...............................205

6.4.2 Prestressed Concrete Specimens ................................................205

6.4.2.1 Specimen PC6N ..........................................................208

6.4.2.1.1 Introduction ...........................................208

6.4.2.1.2 Deflections............................................208

6.4.2.1.3 Longitudinal Mild Steel Strains ..............208

6.4.2.1.4 Strand Strains ........................................209 
6.4.2.1.5 Concrete Surface Strains .......................209

6.4.2.1.6 Failure Observations.............................209

6.4.2.2 Specimen PC6S............................................................ 210

6.4.2.2.1 Introduction .........................................210

6.4.2.2.2 Deflections.............................................210

6.4.2.2.3 Longitudinal Mild Steel Strains ..............211

6.4.2.2.4 Strand Strains ...........................................211

6.4.2.2.5 Stirrup Strains........................................211

6.4.2.2.6 Concrete Surface Strains .........................211

6.4.2.2.7 Failure Observations...............................212

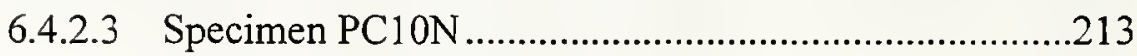

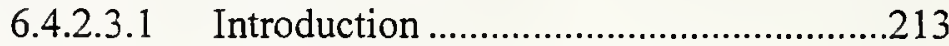

6.4.2.3.2 Deflections..............................................213

6.4.2.3.3 Longitudinal Mild Steel Strains .............213

6.4.2.3.4 Concrete Surface Strains ..........................214

6.4.2.3.5 Failure Observations..............................214

6.4.2.4 Specimen PC10S...................................................215

6.4.2.4.1 Introduction .........................................215

6.4.2.4.2 Deflections............................................215

6.4.2.4.3 Longitudinal Mild Steel Strains ...............216

6.4.2.4.4 Stirrup Strains.........................................216

6.4.2.4.5 Concrete Surface Strains ........................216

6.4.2.4.6 Failure Observations...............................217

6.5 Code-Estimates of Shear Capacity ………….......................................217

6.5.1 Reinforced Concrete Specimens ..................................................218

6.5.2 Prestressed Concrete Specimens ..............................................221

CHAPTER 7: SUMMARY, CONCLUSIONS, AND RECOMMENDATIONS...........587

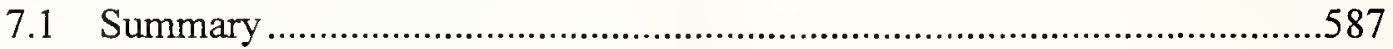

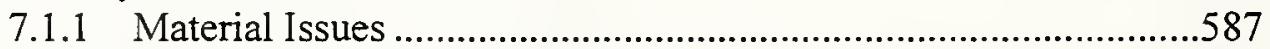

7.1.2 Beam Shear Strength............................................................58

7.1.2.1 Reinforced Concrete Specimens ....................................588

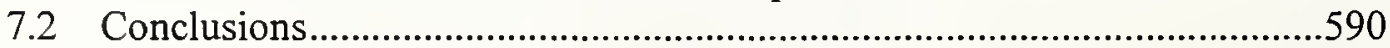

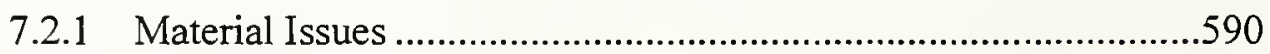

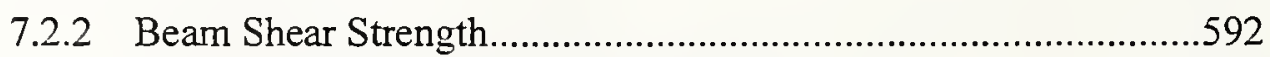

7.2.2.1 Reinforced Concrete Specimens ...................................592

7.2.2.2 Prestressed Concrete Specimens.....................................594

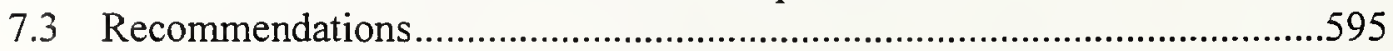

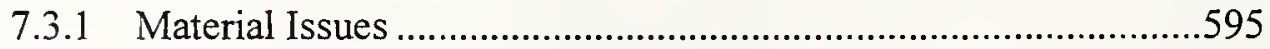

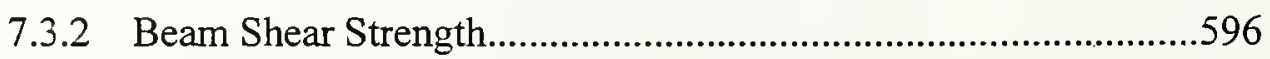

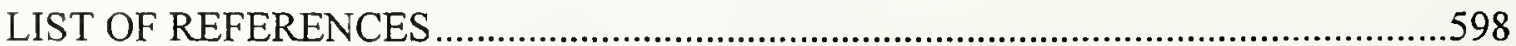


Appendix A: AASHTO-LRFD Tables .

603

Appendix B: Derivation of AASHTO LRFD Equations...................................605 


\section{LIST OF TABLES}

Table

2.1 Requirements of ASTM C 330, C 331, and C 332 for Dry Loose Unit Weight of Lightweight Aggregates . .36

2.2 Specimen Details and Results of Beams Tested by Walraven and Al-Zubi..........37

2.2 Re-examination of the Results of the Beams Tested by Walraven and Al-Zubi ...38

4.1 Requirements of ASTM C 330, C 331, and C 332 for Dry Loose Unit Weight of Lightweight Aggregates ...............................................................................

4.2 Chemical Analysis for Haydite Lightweight Aggregate........................................76

4.3 Gradation and Unit Weight of Haydite Lightweight Aggregate...........................76

4.4 Size Distribution of Haydite Aggregate Determined in the Laboratory .................77

4.5 Size Distribution of Minergy Aggregate Determined in the Laboratory ................77

4.6 Chemical and Physical Properties of Minergy Lightweight Aggregate .................77

4.7 Physical Properties of Lightweight Aggregates Determined in the Laboratory ....78

4.8 Chemical Analysis for Lone Star Type III Cement ………................................78

4.9 Physical Analysis for Lone Star Type III Cement ..............................................78

4.10 Chemical Analysis for Lone Star Type I Cement .................................................79

4.11 Physical Analysis for Lone Star Type I Cement................................................79

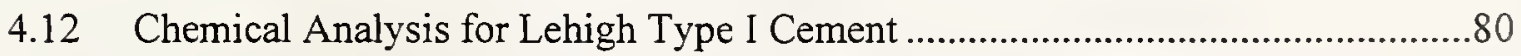

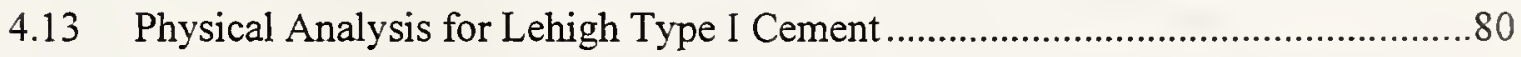

4.14 Chemical Analysis for Montrose Class C Fly Ash, Identification \# 95-031 ........81

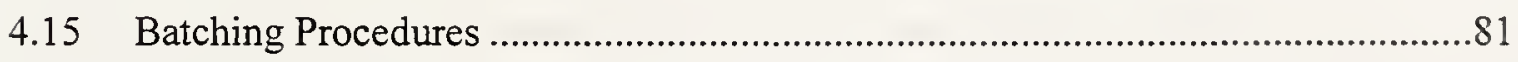

4.16 Proposed Mixes for Use in Bridge Girders and Decks ....................................81 
4.17 Mixture Proportions for $42 \mathrm{MPa}$ (6000 psi) Concrete Using Type III Cement for Use in Bridge Girders

4.18 Mixture Proportions for NWC and SM-LWC, $69 \mathrm{MPa}$ (10,000 psi) Using Type III Cement, Girder Mixes

4.19 Mixture Proportions for High Strength LWC, $69 \mathrm{Mpa}(10,000 \mathrm{psi})$ Using Type III

Cement, Girder Mixes. .83

4.20 Mixture Proportions for LWC with Silica Fume and Fly Ash, $69 \mathrm{MPa}(10,000$ psi), Type III Cement, Girder Mixes

4.21 Mixture Proportions for LWC and NWC, $69 \mathrm{MPa}$ (10,000 psi), Type I Cement, Girder Mixes 84

4.22 Mixture Proportions for LWC and NWC, Bridge Deck, Type I Cement. 84

4.23 Mixture Proportions for Reference LWC and NWC Bridge Deck, Type I

Cement .85

4.24 Concrete Mix Proportions of Reinforced Concrete Series ...................................86

4.25 Hardened Concrete Properties of Reinforced Concrete Series .............................87

4.26 Concrete Mix Proportions of Prestressed Concrete Series .....................................88

4.27 Hardened Concrete Properties of Prestressed Concrete Series...............................8

4.28 Specimen Details of Reinforced Concrete Beam Series.......................................89

4.29 Specimen Details of Prestressed Concrete Beam Series........................................90

5.1 Specifications Followed During Testing of Fresh Concrete Properties................ 149

5.2 Specifications Followed During Testing of Hardened Concrete Properties........149

6.1 Slump, Unit Weight and Air Content for Girder Mixes, $42 \mathrm{MPa}$ (6000 psi) ......224

6.2 Slump, Unit Weight and Air Content for Girder Mixes, $69 \mathrm{MPa}$ (6000 psi) ......224

6.3 Slump, Unit Weight and Air Content for Deck Mixes .....................................224

6.4 Comparison of Slump, Unit Weight and Air Content for $69 \mathrm{MPa}(10,000 \mathrm{psi})$ Girder Mixes with Type III and Type I Cements 
6.5 Compressive Strength for $42 \mathrm{MPa}$ (6000 psi) Girder Mixes

6.6 Compressive Strength for $69 \mathrm{MPa}(10,000 \mathrm{psi})$ Girder Mixes

6.7 Compressive Strength of Concrete With Type III and Type I Cements. $69 \mathrm{MPa}$ $(10,000 \mathrm{psi})$ Girder Mixes

6.8 Compressive Strength for Deck Mixes .226

6.9 Flexural Strength of $42 \mathrm{MPa}(6000 \mathrm{psi})$ Girder Mixes .226

6.10 Flexural Strength of $69 \mathrm{MPa}(10,000 \mathrm{psi})$ Girder Mixes .227

6.11 Flexural Strength for Deck Mixes. .227

6.12 Split Tensile Strength of $42 \mathrm{MPa}(6000 \mathrm{psi})$ Girder Mixes .227

6.13 Split Tensile Strength of $69 \mathrm{MPa}(10,000 \mathrm{psi})$ Girder Mixes .227

6.14 Split Tensile Strength for Deck Mixes. .228

6.15 Static and Dynamic Moduli of Elasticity, and Poisson's Ratio. 42 $\mathrm{MPa}$ (6000 psi) Girder Mixes .228

6.16 Static and Dynamic Moduli of Elasticity, and Poisson's Ratio. 69 $\mathrm{MPa}(10,000$ psi) Girder Mixes 228

6.17 Static and Dynamic Moduli of Elasticity, Poisson's Ratio for Deck Mixes 229

6.18 Density of Hardened Concrete, $42 \mathrm{MPa}$ (6000 psi) Girder Mixes 229

6.19 Density of Hardened Concrete, $69 \mathrm{MPa}$ (10,000 psi) Girder Mixes 229

6.20 Density of Hardened Concrete, Deck Mixes .230

6.21 Data from Modified Point Count Experiment (Deck Mixes) 231

6.22 Air Void Parameters of Hardened Concrete and Air Content of Fresh Concrete (Deck Mixes) .232

6.23 Freeze-Thaw Resistance of Bridge Girder Mixes, $42 \mathrm{MPa}$ (6000 psi).... 233

6.24 Freeze-Thaw Resistance of Bridge Girder Mixes, $69 \mathrm{MPa}(10,000 \mathrm{psi})$ 233 
6.25 Freeze-Thaw Resistance for Newly Developed Bridge Deck Mixes with No Air Drying Prior to Testing.

6.26 Freeze-Thaw Resistance for Newly Developed LWC for Bridge Deck Application with Air Drying Prior to Testing 234

6.27 Freeze-Thaw Resistance for Reference Mixes for Bridge Deck Application with Air Drying Prior to Testing.

6.28 Rapid Chloride Ion Permeability Results for Bridge Girder Mixes, 42 MPa (6000 psi)

6.29 Rapid Chloride Ion Permeability Results for Bridge Girder Mixes, $69 \mathrm{MPa}$ $(10,000 \mathrm{psi})$

6.30 Rapid Chloride Ion Permeability for Bridge Deck Mixes .................................236

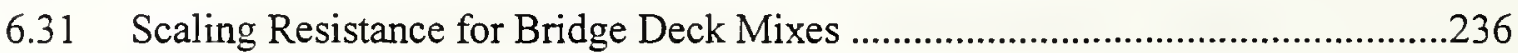

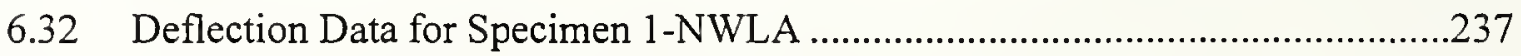

6.33 Longitudinal Steel Strain Data for Specimen 1-NWLA ....................................238

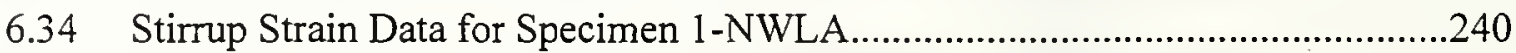

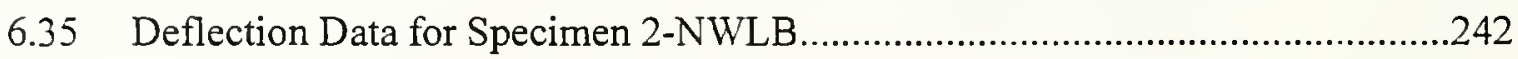

6.36 Longitudinal Strain Data for Specimen 2-NWLB ............................................243

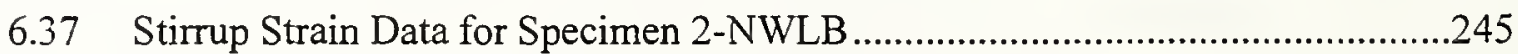

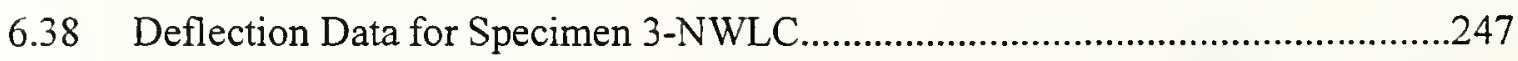

6.39 Longitudinal Steel Strain Data for Specimen 3-NWLC ....................................248

6.40 Stirrup Strain Data for Specimen 3-NWLC ....................................................250

6.41 Deflection Data for Specimen 4-NWLD ......................................................252

6.42 Longitudinal Steel Strain Data for Specimen 4-NWLD ....................................253

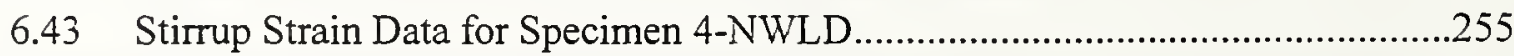

6.44 Deflection Data for Specimen 5-LWLA ………….........................................256 
6.45 Longitudinal Steel Strain Data for Specimen 5-LWLA ....

6.46 Stirrup Strain Data for Specimen 5-LWLA _................................................259

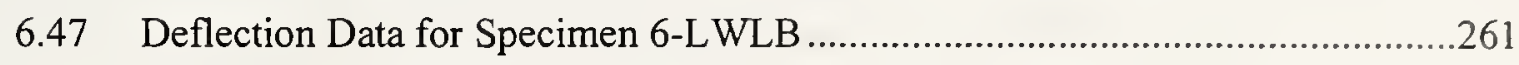

6.48 Longitudinal Steel Strain Data for Specimen 6-LWLB.....................................262

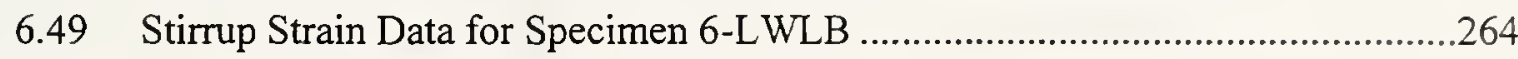

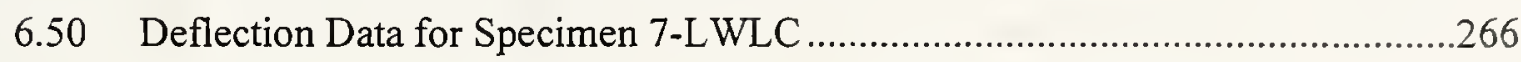

6.51 Longitudinal Steel Strain Data for Specimen 7-LWLC.....................................267

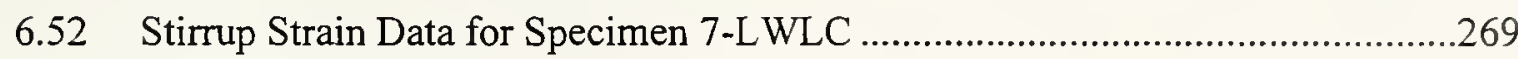

6.53 Deflection Data for Specimen 8-LWLD ........................................................271

6.54 Longitudinal Steel Strain Data for Specimen 8-LWLD ...................................272

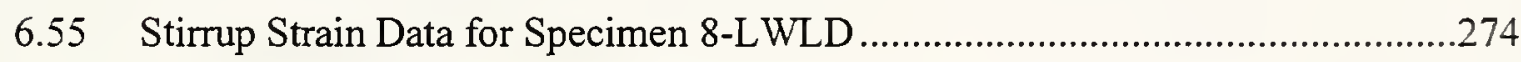

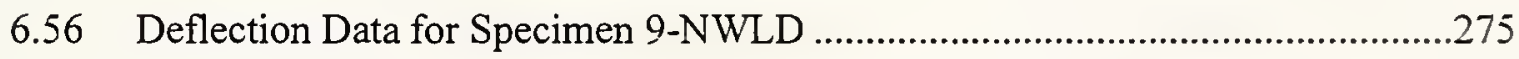

6.57 Longitudinal Steel Strain Data for Specimen 9-NWLD ...................................22

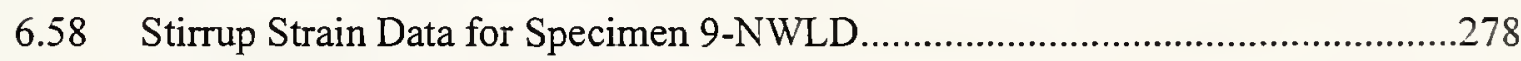

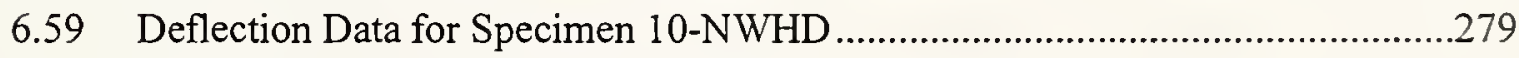

6.60 Longitudinal Steel Strain Data for Specimen 10-NWHD.................................280

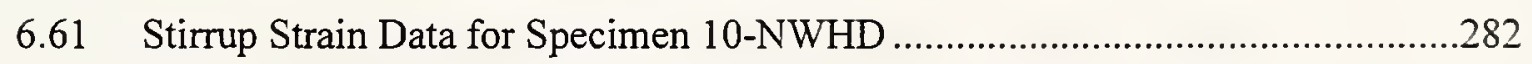

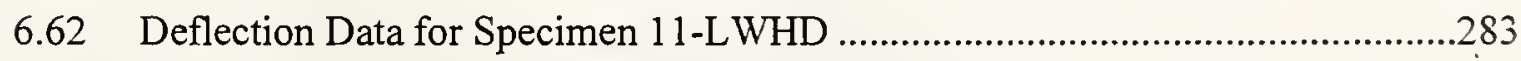

6.63 Longitudinal Steel Strain Data for Specimen 11-LWHD ...................................284

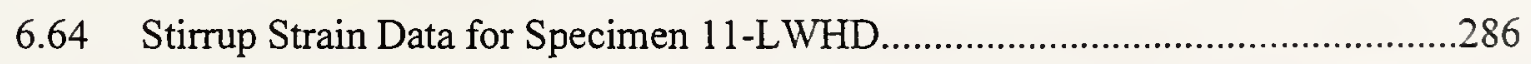

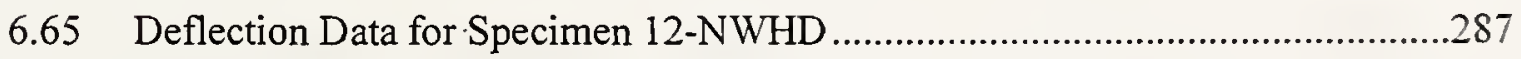

6.66 Longitudinal Steel Strain Data for Specimen 12-NWHD.................................288

6.67 Stirrup Strain Data for Specimen 12-NWHD ................................................290 
6.68 Horizontal Whittemore Strain Data for Specimen 1-NWLA .291

6.69 Vertical Whittemore Strain Data for Specimen 1-NWLA .................................293

6.70 Diagonal Whittemore Strain Data for Specimen 1-NWLA .................................294

6.71 Horizontal Whittemore Strain Data for Specimen 2-NWLB..............................296

6.72 Vertical Whittemore Strain Data for Specimen 2-NWLB .................................298

6.73 Diagonal Whittemore Strain Data for Specimen 2-NWLB ...............................299

6.74 Horizontal Whittemore Strain Data for Specimen 3-NWLC................................301

6.75 Vertical Whittemore Strain Data for Specimen 3-NWLC ...................................303

6.76 Diagonal Whittemore Strain Data for Specimen 3-NWLC ...............................304

6.77 Horizontal Whittemore Strain Data for Specimen 4-NWLD ...............................306

6.78 Vertical Whittemore Strain Data for Specimen 4-NWLD...................................308

6.79 Diagonal Whittemore Strain Data for Specimen 4-NWLD ................................309

6.80 Horizontal Whittemore Strain Data for Specimen 5-LWLA .................................311

6.81 Vertical Whittemore Strain Data for Specimen 5-LWLA ..................................313

6.82 Diagonal Whittemore Strain Data for Specimen 5-L WLA ……………….........314

6.83 Horizontal Whittemore Strain Data for Specimen 6-LWLB ...............................316

6.84 Vertical Whittemore Strain Data for Specimen 6-LWLB ………………........318

6.85 Diagonal Whittemore Strain Data for Specimen 6-LWLB....................................321

6.86 Horizontal Whittemore Strain Data for Specimen 7-LWLC ...............................323

6.87 Vertical Whittemore Strain Data for Specimen 7-L WLC ...................................325

6.88 Diagonal Whittemore Strain Data for Specimen 7-LWLC.................................326

6.89 Horizontal Whittemore Strain Data for Specimen 8-LWLD ...............................328

6.90 Vertical Whittemore Strain Data for Specimen 8-LWLD ...................................330 
6.91 Diagonal Whittemore Strain Data for Specimen 8-LWLD

6.92 Horizontal Whittemore Strain Data for Specimen 9-NWLD ............................333

6.93 Vertical Whittemore Strain Data for Specimen 9-NWLD .................................335

6.94 Diagonal Whittemore Strain Data for Specimen 9-NWLD ................................336

6.95 Horizontal Whittemore Strain Data for Specimen 10-NWHD ...........................338

6.96 Vertical Whittemore Strain Data for Specimen 10-NWHD ...............................340

6.97 Diagonal Whittemore Strain Data for Specimen 10-NWHD ..............................341

6.98 Whittemore Strain Data for Specimen 1 1-LWHD.............................................343

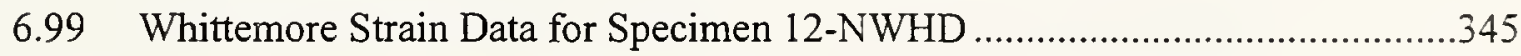

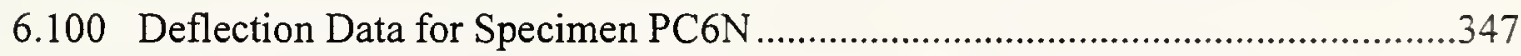

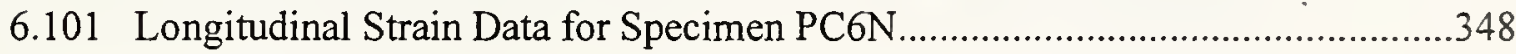

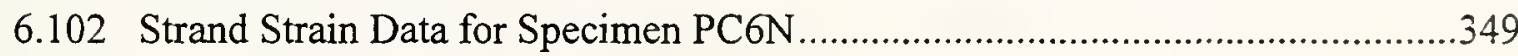

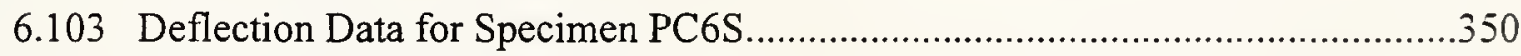

6.104 Longitudinal Steel Strain Data for Specimen PC6S .........................................351

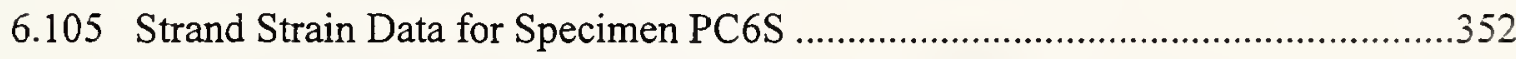

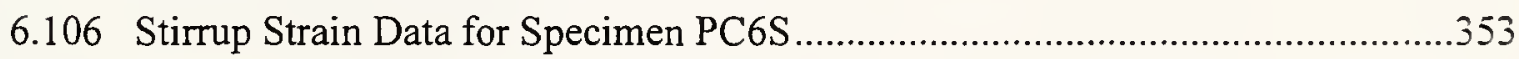

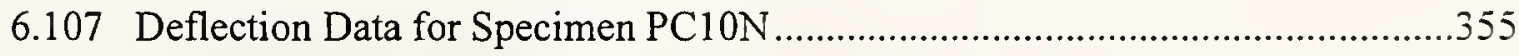

6.108 Longitudinal Steel Strain Data for Specimen PC10N.......................................356

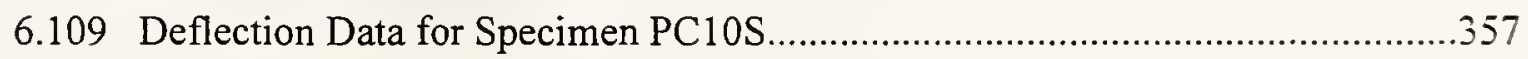

6.110 Longitudinal Steel Strain Data for Specimen PC10S ..........................................358

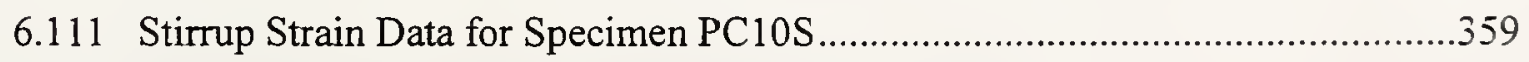

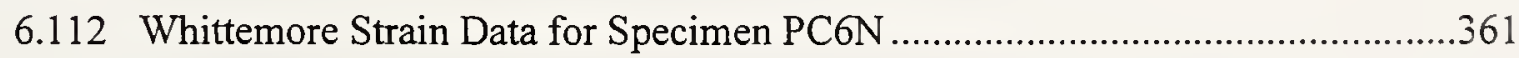

6.113 Whittemore Strain Data for Specimen PC6S ...................................................... 
6.114 Whittemore Strain Data for Specimen PC10N

6.115 Whittemore Strain Data for Specimen PC10S..................................................363

6.116 Comparison of Measured Shear Capacities and Code-Estimated Nominal Shear Capacities, Reinforced Concrete Specimens

6.117 Comparison of Measured Shear Capacities and Code-Estimated Nominal Shear Capacities, Prestressed Concrete Specimens 364

A.1 Reproduction of Table 5.8.3.4.2-1 of 1994 AASHTO LRFD with 1997 Interim Revisions..... 603

A.2 Reproduction of Table 5.8.3.4.2-2 of 1994 AASHTO LRFD with 1997 Interim Revisions. 604

B.1 Reproduction of Table 7-3 of Collins and Mitchell Values of $\theta$ and $\beta$ for Members with Web Reinforcement 614

B.2 Reproduction of Table 7-4 of Collins and Mitchell Values of $\theta$ and $\beta$ for Members without Web Reinforcement .615 


\section{LIST OF FIGURES}

Figure

2.1 Specimen and Test Setup used by Walraven and Al-Zubi 39

2.2 Comparison of Lightweight and Normal Weight Shear Strengths 40

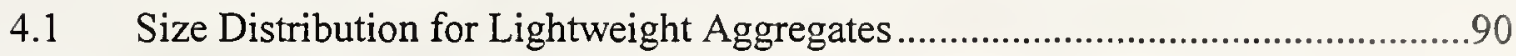

4.2 Mixture Development Program of Concrete for Use in Bridge Girders................91

4.3 Mixture Development Program of Concrete for Use in Bridge Decks..................91

4.4 Stress-Strain Relationship for No. 8 Bars in Reinforced Concrete Series.............92

4.5 Stress-Strain Relationship for No. 9 Bars in Reinforced Concrete Series .............92

4.6 Stress-Strain Relationship for No. 3 Stirrups in Reinforced Concrete Series........93

4.7 Stress-Strain Relationship for No. 7 Bars in Prestressed Concrete Series..............93

4.8 Stress-Strain Relationship for No. 8 Bars in Prestressed Concrete Series..............94

4.9 Stress-Strain Relationship for No. 3 Stirrups in Prestressed Concrete Series .......94

4.10 Idealized Stress-Strain Relationship for $1 / 2$ Inch Special Low-Relaxation

7-Wire Prestressing Strand .95

4.11 Nominal Dimensions and Reinforcement Details for Specimens

1-NWLA and 5-LWLA. 96

4.12 Nominal Dimensions and Reinforcement Details for Specimens

2-NWLB and 6-LWLB.

4.13 Nominal Dimensions and Reinforcement Details for Specimens

3-NWLC and 7-LWLC .98

4.14 Nominal Dimensions and Reinforcement Details for Specimens 4-NWLD, 8-LWLD, 9-NWLD, 10-NWHD, 11-LWHD, and 12-NWHD .99

4.15 Cross Sections of Reinforcing Details in Test Regions ......................................100

4.16 Consolidating Concrete with Handheld Vibrators .............................................101 
4.17 Sampling of Concrete at Precast Plant. 101

4.18 Samples and Formwork for Specimen 10-NWHD in Laboratory .......................102

4.19 Making Test Samples for Specimen 10-NWHD in Laboratory..........................102

4.20 Cross Sectional Dimensions of Prestressed Concrete Specimens ........................103

4.21 Cross Sectional Reinforcement Details of Specimen PC6S at Midspan .............104

4.22 Cross Sectional Reinforcement Details of Specimen PC@N at Midspan..............104

4.23 Cross Sectional Reinforcement Details of Specimen PC10S at Midspan ...........105

4.24 Cross Sectional Reinforcement Details of Specimen PC10N at Midspan...........105

4.25 Reinforcement Details for Specimens PC6N and PC10N …….........................106

4.26 Reinforcement Details for Specimens PC6S and PC10S ..............................107

4.27 Specimen PC6N Prior to Casting at the Precast Plant .......................................108

4.28 Specimen PC6S Prior to Casting at the Precast Plant..........................................108

4.29 Flame-Cutting Strands to Release Prestress Force .............................................109

4.30 Falsework for Cast-In-Place Slab ….............................................................110

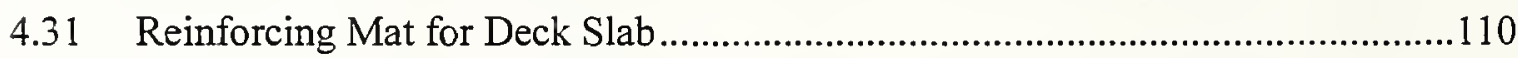

4.32 Location of LVDTs and Dial Gages for Reinforced Concrete Series .................111

4.33 LVDTs on West Side of Typical Reinforced Concrete Specimen .......................111

4.34 Strain Gage Locations for Specimens 1-NWLA and 5-LWLA...........................112

4.35 Strain Gage Locations for Specimens 2-NWLB and 6-LWLB .........................113

4.36 Strain Gage Locations for Specimens 3-NWLC and 7-LWLC ...........................114

4.37 Strain Gage Locations for Specimens 4-NWLD, 8-LWLD, 9-NWLD, 10-NWHD, 11-LWHD, and 12-NWHD

4.38 Data Acquisition Unit 
4.39 Dial Gages Underneath East Side of Reinforced Concrete Specimen

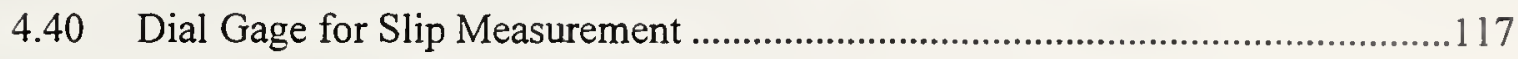

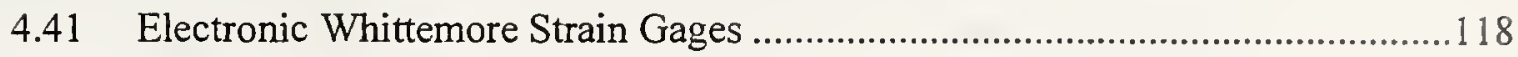

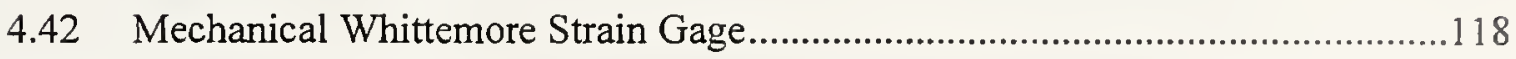

4.43 Location of Whittemore Targets for Specimens 1-NWLA, 2-NWLB, 3-NWLC, 5-LWLA, 9-NWLD, and 10-NWHD.

4.44 Location of Horizontal and Vertical Electronic Whittemore Gage Measurements for Specimens 1-NWLA, 2-NWLB, 3-NWLC, 5-LWLA, 9-NWLD, and 10-NWHD.

4.45 Location of Diagonal Electronic Whittemore Gage Measurements for Specimens 1-NWLA, 2-NWLB, 3-NWLC, 5-LWLA, 9-NWLD, and 10-NWHD .

4.46 Location of Whittemore Targets for Specimens 4-NWLD, 7-LWLC, and 8-LWLD.

4.46 Location of Horizontal and Vertical Electronic Whittemore Gage Measurements for Specimens 4-NWLD, 7-LWLC, and 8-LWLD

4.47 Location of Diagonal Electronic Whittemore Gage Measurements for Specimens 4-NWLD, 7-LWLC, and 8-LWLD.

4.49 Location of Whittemore Targets for Specimen 6-LWLB 125

4.50 Location of Horizontal and Vertical Electronic Whittemore Gage Measurements for Specimen 6-LWLB 126

4.51 Location of Diagonal Electronic Whittemore Gage Measurements for Specimen 6-LWLB

4.52 Location of Whittemore Rosettes and Measurements for Specimens 11-LWHD

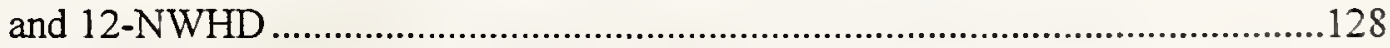

4.53 Location of LVDTs for Prestressed Concrete Series .........................................129

4.54 Location of Longitudinal Strain Gages in Specimen PC6S at Midspan ..............130

4.55 Location of Longitudinal Strain Gages in Specimen PC6N at Midspan .............130

4.56 Location of Longitudinal Strain Gages in Specimen PC10S at Midspan............131 
4.57 Location of Longitudinal Strain Gages in Specimen PC10N at Midspan

4.58 Location of Stirrup Strain Gages in Specimens PC6S and PC10S.....................132

4.59 East Side of Prestressed Concrete Setup (PC6S) Showing Location of Dial Gages

4.60 Dial Gage for Strand Slip Measurements

4.61 Location and Identification of Whittemore Rosettes and Measurements, Specimens PC6N and PC6S

4.62 Location and Identification of Whittemore Rosettes and Measurements, Specimens PC10N and PCIOS

5.1 Compression Testing of Concrete Cylinders .................................................150

5.2 Concrete Beam being Loaded by The Simple Third Point Method......................150

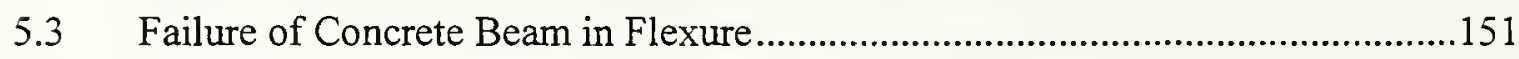

5.4 Tensile Splitting Test of Concrete Sample ....................................................151

5.5 Test Setup for Static Modulus of Elasticity and Poisson's Ratio .........................152

5.6 Testing for Static Modulus of Elasticity and Poisson's Ratio ............................152

5.7 Testing of $6.6 \mathrm{~cm} \times 6.6 \mathrm{~cm} \times 33 \mathrm{~cm}$ (3in. x 3in. x 1 in.) Beams for Dynamic Modulus of Elasticity Using Grindo-Sonic Non-destructive Materials Testing System

5.8 Air Void Distribution Determination Using Modified Point Count Method........153

5.9 Testing of $6.6 \mathrm{~cm} \times 6.6 \mathrm{~cm} \times 33 \mathrm{~cm}$ (3in. $\times 3$ in. $\times 15$ in.) Beams in Freezing and

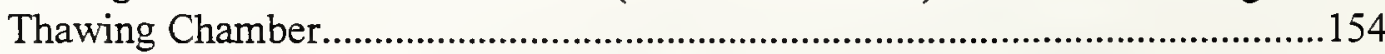

5.10 Rapid Chloride Ion Permeability Testing of Concrete Prizms ............................154

5.11 Resistance to Scaling Testing of Concrete Samples in Freezing Chamber .........155

5.12 Test Setup for Reinforced Concrete Series ........................................................156

5.13 North End of Test Setup ................................................................................157 
5.14 Support Beam and Load Cells at North End of Typical Reinforced

Concrete Specimen (East Face)

5.15 Loading Point at Maximum Positive Moment Section of Typical

Reinforced Concrete Specimen (East Face)

5.16 Shear Force and Bending Moment Diagrams for Reinforced Concrete

Specimens

5.17 Test Setup for Prestressed Concrete Beam Series

5.18 West Side of Prestressed Concrete Series Setup.

5.19 Support Beam and LVDT at South End of Prestressed Concrete Specimen .......161

6.1 Compressive Strength of $42 \mathrm{MPa}(6000 \mathrm{psi})$ Girder Mixes ...............................365

6.2 Compressive Strength of 69 MPA (10,000 psi) Girder Mixes ............................365

6.3 Effect of Mineral Admixtures on Compressive Strength of $69 \mathrm{MPa}(10,000$

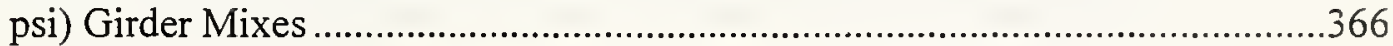

6.4 Comparison of Compressive Strength of Concrete Made with Type III and Type I Cements, $69 \mathrm{MPa}$ (6000 psi) Girder Mixes

6.5 Compressive Strength Comparison for Newly Developed Mixes and Reference

Mixes for Use in Bridge Decks

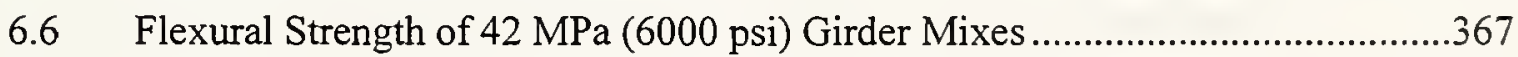

6.7 Flexural Strength of $69 \mathrm{MPa}(10,000 \mathrm{psi})$ Girder Mixes ...................................368

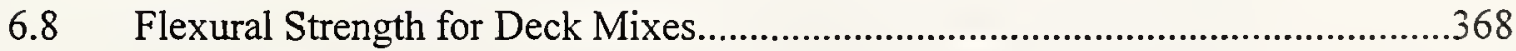

6.9 Split Tensile Strength of $42 \mathrm{MPa}$ (6000 psi) Girder Mixes ................................369

6.10 Split Tensile Strength of $69 \mathrm{MPa}(10,000 \mathrm{psi})$ Girder Mixes ..............................369

6.11 Split Tensile Strength for Deck Mixes...............................................................370

6.12 Static and Dynamic Moduli of Elasticity for $42 \mathrm{MPa}$ (6000 psi) Girder Mixes...370

6.13 Static and Dynamic Moduli of Elasticity $69 \mathrm{MPa}(10,000 \mathrm{psi})$ Girder Mixes ....371

6.14 Comparison of Moduli of Elasticity for Deck Mixes 
6.15 Density of Hardened Concrete, Girder Mixes

6.16 Density of Hardened Concrete, Deck Mixes

6.17 Temperature Variation in $69 \mathrm{MPa}(10,000 \mathrm{psi}) \mathrm{LWC}$ Girder Mixes .373

6.18 Comparison of Spacing Factors for Deck Concrete Mixes 374

6.19 Comparison of Air Content for Hardened and Fresh Concrete, Newly Developed Deck Mixes

6.20 Comparison of Air Content for Hardened and Fresh Concrete, Reference Deck Mixes

6.21 Drying Shrinkage for Bridge Girder Mixes ....................................................375

6.22 Drying Shrinkage for Newly Developed Bridge Deck Mixes .............................376

6.23 Drying Shrinkage for Reference Deck Mixes....................................................376

6.24 Comparison of Durability Factors for $42 \mathrm{MPa}$ (6000 psi) Mixes .........................377

6.25 Comparison of Durability Factors for $69 \mathrm{MPa}(10,000$ psi) Mixes......................377

6.26 Comparison of Durability Factors for Newly Developed Bridge Deck Mixes with No Air Drying Prior to Testing.

6.27 Comparison of Durability Factors for Newly Developed LWC for Bridge Deck Application with Air Drying Prior to Testing .378

6.28 Comparison of Durability Factors for Reference Mixes for Bridge Deck Application with Air Drying Prior to Testing.

6.29 Comparison of Durability Factors for Newly Developed and Reference Mixes for Bridge Deck Application

6.30 Effect of Air Drying period of LWC Samples on Durability Factor..... 380

6.31 Comparison of 28 Day Coulomb Reading for Girder Mixes, $42 \mathrm{MPa}(6000$ psi)

6.32 Comparison of 28 and 56 Day Coulomb Reading for Girder Mixes, $69 \mathrm{MPa}$ $(10,000 \mathrm{psi})$

6.33 Comparison of 28 and 56 Day Coulomb Reading for Deck Mixes 381 
6.34 Scaling Resistance Ratings Observed for Deck Concrete

6.35 Shear-Deflection Relationship, Specimen 1-NWLA …....................................383

6.36 Shear-Strain Relationship, Specimen 1-NWLA, Section 1, Bottom Steel ..........383

6.37 Shear-Strain Relationship, Specimen 1-NWLA, Section 2, Bottom Steel ..........384

6.38 Shear-Strain Relationship, Specimen 1-NWLA, Section 3, Bottom Steel ...........384

6.39 Shear-Strain Relationship, Specimen 1-NWLA, Section 4, Top Steel.................385

6.40 Shear-Stirrup Strain Relationship, Specimen 1-NWLA ....................................385

6.41 Shear-Deflection Relationship, Specimen 2-NWLB .......................................386

6.42 Shear-Strain Relationship, Specimen 2-NWLB, Section 1, Bottom Steel ...........386

6.43 Shear-Strain Relationship, Specimen 2-NWLB, Section 2, Bottom Steel ...........387

6.44 Shear-Strain Relationship, Specimen 2-NWLB, Section 3, Bottom Steel ..........387

6.45 Shear-Strain Relationship, Specimen 2-NWLB, Section 4, Top Steel ..................388

6.46 Shear-Stirrup Strain Relationship, Specimen 2-NWLB ......................................388

6.47 Shear-Deflection Relationship, Specimen 3-NWLC ..........................................389

6.48 Shear-Strain Relationship, Specimen 3-NWLC, Section 1, Bottom Steel ...........389

6.49 Shear-Strain Relationship, Specimen 3-NWLC, Section 2, Bottom Steel ...........390

6.50 Shear-Strain Relationship, Specimen 3-NWLC, Section 3, Bottom Steel ..........390

6.51 Shear-Strain Relationship, Specimen 3-NWLC, Section 4, Top Steel .................391

6.52 Shear-Stirrup Strain Relationship, Specimen 3-NWLC .....................................391

6.53 Shear-Deflection Relationship, Specimen 4-NWLD .........................................392

6.54 Shear-Strain Relationship, Specimen 4-NWLD, Section 1, Bottom Steel ...........392

6.55 Shear-Strain Relationship, Specimen 4-NWLD, Section 2, Bottom Steel ...........393

6.56 Shear-Strain Relationship, Specimen 4-NWLD, Section 3, Bottom Steel ...........393 
6.57 Shear-Strain Relationship, Specimen 4-NWLD, Section 4, Top Steel. 394

6.58 Shear-Stirrup Strain Relationship, Specimen 4-NWLD ....................................394

6.59 Shear-Deflection Relationship, Specimen 5-LWLA ……………………..........395

6.60 Shear-Strain Relationship, Specimen 5-LWLA, Section 1, Bottom Steel............395

6.61 Shear-Strain Relationship, Specimen 5-LWLA, Section 2, Bottom Steel.............396

6.62 Shear-Strain Relationship, Specimen 5-LWLA, Section 3, Bottom Steel............396

6.63 Shear-Strain Relationship, Specimen 5-LWLA, Section 4, Top Steel .................397

6.64 Shear-Stirrup Strain Relationship, Specimen 5-LWLA A.....................................397

6.65 Shear-Deflection Relationship, Specimen 6-LWLB .............................................398

6.66 Shear-Strain Relationship, Specimen 6-LWLB, Section 1, Bottom Steel............398

6.67 Shear-Strain Relationship, Specimen 6-LWLB, Section 2, Bottom Steel............399

6.68 Shear-Strain Relationship, Specimen 6-LWLB, Section 3, Bottom Steel............399

6.69 Shear-Strain Relationship, Specimen 6-LWLB, Section 4, Top Steel .................400

6.70 Shear-Stirrup Strain Relationship, Specimen 6-LWLB ……………………........400

6.71 Shear-Deflection Relationship, Specimen 7-LWLC.............................................401

6.72 Shear-Strain Relationship, Specimen 7-LWLC, Section 1, Bottom Steel ...........401

6.73 Shear-Strain Relationship, Specimen 7-LWLC, Section 2, Bottom Steel...........402

6.74 Shear-Strain Relationship, Specimen 7-L WLC, Section 3, Bottom Steel...........402

6.75 Shear-Strain Relationship, Specimen 7-LWLC, Section 4, Top Steel ................403

6.76 Shear-Stirrup Strain Relationship, Specimen 7-LWLC......................................403

6.77 Shear-Deflection Relationship, Specimen 8-LWLD ...........................................404

6.78 Shear-Strain Relationship, Specimen 8-LWLD, Section 1, Bottom Steel...........404

6.79 Shear-Strain Relationship, Specimen 8-LWLD, Section 2, Bottom Steel............405 
6.80 Shear-Strain Relationship, Specimen 8-LWLD, Section 3, Bottom Steel......... 405

6.81 Shear-Strain Relationship, Specimen 8-LWLD, Section 4, Top Steel ................406

6.82 Shear-Stirrup Strain Relationship, Specimen 8-LWLD.......................................406

6.83 Shear-Deflection Relationship, Specimen 9-NWLD ........................................407

6.84 Shear-Strain Relationship, Specimen 9-NWLD, Section 1, Bottom Steel ..........407

6.85 Shear-Strain Relationship, Specimen 9-NWLD, Section 2, Bottom Steel ..........408

6.86 Shear-Strain Relationship, Specimen 9-NWLD, Section 3, Bottom Steel ..........408

6.87 Shear-Strain Relationship, Specimen 9-NWLD, Section 4, Top Steel.................409

6.88 Shear-Stirrup Strain Relationship, Specimen 9-NWLD ......................................409

6.89 Shear-Deflection Relationship, Specimen 10-NWHD ....................................410

6.90 Shear-Strain Relationship, Specimen 10-NWHD, Section 1, Bottom Steel........410

6.91 Shear-Strain Relationship, Specimen 10-NWHD, Section 2, Bottom Steel........411

6.92 Shear-Strain Relationship, Specimen 10-NWHD, Section 3, Bottom Steel........411

6.93 Shear-Strain Relationship, Specimen 10-NWHD, Section 4, Top Steel .............412

6.94 Shear-Stirrup Strain Relationship, Specimen 10-NWHD...................................412

6.95 Shear-Deflection Relationship, Specimen 11-LWHD …………………...........413

6.96 Shear-Strain Relationship, Specimen 11-LWHD, Section 1, Bottom Steel ........413

6.97 Shear-Strain Relationship, Specimen 11-LWHD, Section 2, Bottom Steel ........414

6.98 Shear-Strain Relationship, Specimen 11-LWHD, Section 3, Bottom Steel ........414

6.99 Shear-Strain Relationship, Specimen 11-LWHD, Section 4, Top Steel..............415

6.100 Shear-Stirrup Strain Relationship, Specimen 11-LWHD ....................................415

6.101 Shear-Deflection Relationship, Specimen 12-NWHD …..................................416

6.102 Shear-Strain Relationship, Specimen 12-NWHD, Section 1, Bottom Steel........416 
6.103 Shear-Strain Relationship, Specimen 12-NWHD, Section 2, Bottom Steel........417

6.104 Shear-Strain Relationship, Specimen 12-NWHD, Section 3, Bottom Steel........417

6.105 Shear-Strain Relationship, Specimen 12-NWHD, Section 4, Top Steel .............418

6.106 Shear-Stirrup Strain Relationship, Specimen 12-NWHD...................................418

6.107 Shear-Strain Relationship, Horizontal Whittemore Measurements 1-5, Specimen 1-NWLA

6.108 Shear-Strain Relationship, Horizontal Whittemore Measurements 6-10, Specimen 1-NWLA

6.109 Shear-Strain Relationship, Horizontal Whittemore Measurements 11-15, Specimen 1-NWLA

6.110 Shear-Strain Relationship, Horizontal Whittemore Measurements 16-20, Specimen 1-NWLA

6.111 Shear-Strain Relationship, Horizontal Whittemore Measurements 21-25, Specimen 1-NWLA

6.112 Shear-Strain Relationship, Horizontal Whittemore Measurements 26-30, Specimen 1-NWLA

6.113 Shear-Strain Relationship, Vertical Whittemore Measurements 1-3, Specimen 1-NWLA

6.114 Shear-Strain Relationship, Vertical Whittemore Measurements 4-6, Specimen 1-NWLA

6.115 Shear-Strain Relationship, Vertical Whittemore Measurements 7-9, Specimen 1-NWLA

6.116 Shear-Strain Relationship, Vertical Whittemore Measurements 10-12, Specimen 1-NWLA

6.117 Shear-Strain Relationship, Vertical Whittemore Measurements 13-15, Specimen 1-NWLA

6.118 Shear-Strain Relationship, Vertical Whittemore Measurements 16-18, Specimen 1-NWLA 
6.119 Shear-Strain Relationship, Diagonal Whittemore Measurements 1-6, Specimen 1-NWLA

6.120 Shear-Strain Relationship, Diagonal Whittemore Measurements 7-12, Specimen 1-NWLA

6.121 Shear-Strain Relationship, Diagonal Whittemore Measurements 13-18, Specimen 1-NWLA

6.122 Shear-Strain Relationship, Diagonal Whittemore Measurements 19-24, Specimen 1-NWLA

6.123 Shear-Strain Relationship, Diagonal Whittemore Measurements 25-30, Specimen 1-NWLA

6.124 Shear-Strain Relationship, Diagonal Whittemore Measurements 31-36, Specimen 1-NWLA

6.125 Shear-Strain Relationship, Horizontal Whittemore Measurements 1-5, Specimen 2-NWLB

6.126 Shear-Strain Relationship, Horizontal Whittemore Measurements 6-10, Specimen 2-NWLB

6.127 Shear-Strain Relationship, Horizontal Whittemore Measurements 11-15, Specimen 2-NWLB

6.128 Shear-Strain Relationship, Horizontal Whittemore Measurements 16-20, Specimen 2-NWLB

6.129 Shear-Strain Relationship, Horizontal Whittemore Measurements 21-25, Specimen 2-NWLB 430

6.130 Shear-Strain Relationship, Horizontal Whittemore Measurements 26-30, Specimen 2-NWLB

6.131 Shear-Strain Relationship, Vertical Whittemore Measurements 1-3, Specimen 2-NWLB

6.132 Shear-Strain Relationship, Vertical Whittemore Measurements 4-6, Specimen 2-NWLB:

6.133 Shear-Strain Relationship, Vertical Whittemore Measurements 7-9, Specimen 2-NWLB. 
6.134 Shear-Strain Relationship, Vertical Whittemore Measurements 10-12, Specimen 2-NWLB.

6.135 Shear-Strain Relationship, Vertical Whittemore Measurements 13-15, Specimen 2-NWLB.

6.136 Shear-Strain Relationship, Vertical Whittemore Measurements 16-18, Specimen 2-NWLB.

6.137 Shear-Strain Relationship, Diagonal Whittemore Measurements 1-6, Specimen 2-NWLB. 434

6.138 Shear-Strain Relationship, Diagonal Whittemore Measurements 7-12, Specimen 2-NWLB. 434

6.139 Shear-Strain Relationship, Diagonal Whittemore Measurements 13-18, Specimen 2-NWLB. .435

6.140 Shear-Strain Relationship, Diagonal Whittemore Measurements 19-24, Specimen 2-NWLB. .435

6.141 Shear-Strain Relationship, Diagonal Whittemore Measurements 25-30, Specimen 2-NWLB. 436

6.142 Shear-Strain Relationship, Diagonal Whittemore Measurements 31-36, Specimen 2-NWLB. 436

6.143 Shear-Strain Relationship, Horizontal Whittemore Measurements 1-5, Specimen 3-NWLC....

6.144 Shear-Strain Relationship, Horizontal Whittemore Measurements 6-10, Specimen 3-NWLC

6.145 Shear-Strain Relationship, Horizontal Whittemore Measurements 11-15, Specimen 3-NWLC 438

6.146 Shear-Strain Relationship, Horizontal Whittemore Measurements 16-20, Specimen 3-NWLC. .438

6.147 Shear-Strain Relationship, Horizontal Whittemore Measurements 21-25, Specimen 3-NWLC

6.148 Shear-Strain Relationship, Horizontal Whittemore Measurements 26-30, Specimen 3-NWLC.... 
6.149 Shear-Strain Relationship, Vertical Whittemore Measurements 1-3, Specimen 3-NWLC

6.150 Shear-Strain Relationship, Vertical Whittemore Measurements 4-6, Specimen 3-NWLC.

6.151 Shear-Strain Relationship, Vertical Whittemore Measurements 7-9, Specimen 3-NWLC

6.152 Shear-Strain Relationship, Vertical Whittemore Measurements 10-12, Specimen 3-NWLC

6.153 Shear-Strain Relationship, Vertical Whittemore Measurements 13-15, Specimen 3-NWLC.

6.154 Shear-Strain Relationship, Vertical Whittemore Measurements 16-18, Specimen 3-NWLC.

6.155 Shear-Strain Relationship, Diagonal Whittemore Measurements 1-6, Specimen 3-NWLC.

6.156 Shear-Strain Relationship, Diagonal Whittemore Measurements 7-12, Specimen 3-NWLC.

6.157 Shear-Strain Relationship, Diagonal Whittemore Measurements 13-18, Specimen 3-NWLC

6.158 Shear-Strain Relationship, Diagonal Whittemore Measurements 19-24, Specimen 3-NWLC. 444

6.159 Shear-Strain Relationship, Diagonal Whittemore Measurements 25-30, Specimen 3-NWLC.

6.160 Shear-Strain Relationship, Diagonal Whittemore Measurements 31-36, Specimen 3-NWLC

6.161 Shear-Strain Relationship, Horizontal Whittemore Measurements 1-5, Specimen 4-NWLD .446

6.162 Shear-Strain Relationship, Horizontal Whittemore Measurements 6-10, Specimen 4-NWLD 446

6.163 Shear-Strain Relationship, Horizontal Whittemore Measurements 11-15, Specimen 4-NWLD 
6.164 Shear-Strain Relationship, Horizontal Whittemore Measurements 16-20, Specimen 4-NWLD

6.165 Shear-Strain Relationship, Horizontal Whittemore Measurements 21-25, Specimen 4-NWLD

6.166 Shear-Strain Relationship, Horizontal Whittemore Measurements 26-30, Specimen 4-NWLD

6.167 Shear-Strain Relationship, Horizontal Whittemore Measurements 31-35, Specimen 4-NWLD

6.168 Shear-Strain Relationship, Horizontal Whittemore Measurements 36-40, Specimen 4-NWLD

6.169 Shear-Strain Relationship, Vertical Whittemore Measurements 1-2, Specimen 4-NWLD 450

6.170 Shear-Strain Relationship, Vertical Whittemore Measurements 3-4, Specimen 4-NWLD 450

6.171 Shear-Strain Relationship, Vertical Whittemore Measurements 5-6, Specimen 4-NWLD

6.172 Shear-Strain Relationship, Vertical Whittemore Measurements 7-8, Specimen 4-NWLD

6.173 Shear-Strain Relationship, Vertical Whittemore Measurements 9-10, Specimen 4-NWLD

6.174 Shear-Strain Relationship, Vertical Whittemore Measurements 11-12, Specimen 4-NWLD

6.175 Shear-Strain Relationship, Vertical Whittemore Measurements 13-14, Specimen 4-NWLD

6.176 Shear-Strain Relationship, Vertical Whittemore Measurements 15-16, Specimen 4-NWLD

6.177 Shear-Strain Relationship, Vertical Whittemore Measurements 17-18, Specimen 4-NWLD

6.178 Shear-Strain Relationship, Diagonal Whittemore Measurements 1-4, Specimen 4-NWLD 
6.179 Shear-Strain Relationship, Diagonal Whittemore Measurements 5-8, Specimen 4-NWLD

6.180 Shear-Strain Relationship, Diagonal Whittemore Measurements 9-12, Specimen 4-NWLD

6.181 Shear-Strain Relationship, Diagonal Whittemore Measurements 13-16, Specimen 4-NWLD

6.182 Shear-Strain Relationship, Diagonal Whittemore Measurements 17-20, Specimen 4-NWLD

6.183 Shear-Strain Relationship, Diagonal Whittemore Measurements 21-24, Specimen 4-NWLD

6.184 Shear-Strain Relationship, Diagonal Whittemore Measurements 25-28, Specimen 4-NWLD

6.185 Shear-Strain Relationship, Diagonal Whittemore Measurements 29-32, Specimen 4-NWLD 458

6.186 Shear-Strain Relationship, Horizontal Whittemore Measurements 1-5, Specimen 5-LWLA

6.187 Shear-Strain Relationship, Horizontal Whittemore Measurements 6-10, Specimen 5-LWLA

6.188 Shear-Strain Relationship, Horizontal Whittemore Measurements 11-15, Specimen 5-LWLA

6.189 Shear-Strain Relationship, Horizontal Whittemore Measurements 16-20, Specimen 5-LWLA. 460

6.190 Shear-Strain Relationship, Horizontal Whittemore Measurements 21-25, Specimen 5-LWLA 461

6.191 Shear-Strain Relationship, Horizontal Whittemore Measurements 26-30, Specimen 5-LWLA

6.192 Shear-Strain Relationship, Vertical Whittemore Measurements 1-3, Specimen 5-LWLA: 462

6.193 Shear-Strain Relationship, Vertical Whittemore Measurements 4-6, Specimen 5-LWLA 
6.194 Shear-Strain Relationship, Vertical Whittemore Measurements 7-9, Specimen 5-LWLA.

6.195 Shear-Strain Relationship, Vertical Whittemore Measurements 10-12, Specimen 5-LWLA.

6.196 Shear-Strain Relationship, Vertical Whittemore Measurements 13-15, Specimen 5-LWLA.... 464

6.197 Shear-Strain Relationship, Vertical Whittemore Measurements 16-18, Specimen 5-LWLA. .464

6.198 Shear-Strain Relationship, Diagonal Whittemore Measurements 1-6, Specimen 5-LWLA.

6.199 Shear-Strain Relationship, Diagonal Whittemore Measurements 7-12, Specimen 5-LWLA

6.200 Shear-Strain Relationship, Diagonal Whittemore Measurements 13-18, Specimen 5-LWLA.

6.201 Shear-Strain Relationship, Diagonal Whittemore Measurements 19-24, Specimen 5-LWLA.

6.202 Shear-Strain Relationship, Diagonal Whittemore Measurements 25-30, Specimen 5-LWLA.

6.203 Shear-Strain Relationship, Diagonal Whittemore Measurements 31-36, Specimen 5-LWLA.

6.204 Shear-Strain Relationship, Horizontal Whittemore Measurements 1-5, Specimen 6-LWLB .468

6.205 Shear-Strain Relationship, Horizontal Whittemore Measurements 6-10, Specimen 6-LWLB .468

6.206 Shear-Strain Relationship, Horizontal Whittemore Measurements 11-15, Specimen 6-LWLB .469

6.207 Shear-Strain Relationship, Horizontal Whittemore Measurements 16-20, Specimen 6-LWLB 469

6.208 Shear-Strain Relationship, Horizontal Whittemore Measurements 21-25, Specimen 6-LWLB 
6.209 Shear-Strain Relationship, Horizontal Whittemore Measurements 26-30, Specimen 6-LWLB

6.210 Shear-Strain Relationship, Vertical Whittemore Measurements 1-3, Specimen 6-LWLB

6.211 Shear-Strain Relationship, Vertical Whittemore Measurements 4-6, Specimen 6-LWLB

6.212 Shear-Strain Relationship, Vertical Whittemore Measurements 7-9, Specimen 6-LWLB

6.213 Shear-Strain Relationship, Vertical Whittemore Measurements 10-12, Specimen 6-LWLB

6.214 Shear-Strain Relationship, Vertical Whittemore Measurements 13-15, Specimen 6-LWLB

6.215 Shear-Strain Relationship, Vertical Whittemore Measurements 16-18, Specimen 6-LWLB

6.216 Shear-Strain Relationship, Vertical Whittemore Measurements 19-21, Specimen 6-LWLB

6.217 Shear-Strain Relationship, Vertical Whittemore Measurements 22-24, Specimen 6-LWLB

6.218 Shear-Strain Relationship, Vertical Whittemore Measurements 25-27, Specimen 6-LWLB .475

6.219 Shear-Strain Relationship, Vertical Whittemore Measurements 28-30, Specimen 6-LWLB .475

6.220 Shear-Strain Relationship, Vertical Whittemore Measurements 31-33, Specimen 6-LWLB .476

6.221 Shear-Strain Relationship, Vertical Whittemore Measurements 34-36, Specimen 6-LWLB 476

6.222 Shear-Strain Relationship, Vertical Whittemore Measurements 37-39, Specimen 6-LWLB

6.223 Shear-Strain Relationship, Diagonal Whittemore Measurements 1-6, Specimen 6-LWLB 
6.224 Shear-Strain Relationship, Diagonal Whittemore Measurements 7-12, Specimen 6-LWLB

6.225 Shear-Strain Relationship, Diagonal Whittemore Measurements 13-18, Specimen 6-LWLB

6.226 Shear-Strain Relationship, Diagonal Whittemore Measurements 19-24, Specimen 6-LWLB

6.227 Shear-Strain Relationship, Diagonal Whittemore Measurements 25-30, Specimen 6-LWLB 480

6.228 Shear-Strain Relationship, Diagonal Whittemore Measurements 31-36, Specimen 6-LWLB .480

6.229 Shear-Strain Relationship, Horizontal Whittemore Measurements 1-5, Specimen 7-LWLC

6.230 Shear-Strain Relationship, Horizontal Whittemore Measurements 6-10, Specimen 7-LWLC 481

6.231 Shear-Strain Relationship, Horizontal Whittemore Measurements 11-15, Specimen 7-LWLC .482

6.232 Shear-Strain Relationship, Horizontal Whittemore Measurements 16-20, Specimen 7-LWLC .482

6.233 Shear-Strain Relationship, Horizontal Whittemore Measurements 21-25, Specimen 7-LWLC .483

6.234 Shear-Strain Relationship, Horizontal Whittemore Measurements 26-30, Specimen 7-LWLC .483

6.235 Shear-Strain Relationship, Horizontal Whittemore Measurements 31-35, Specimen 7-LWLC 484

6.236 Shear-Strain Relationship, Horizontal Whittemore Measurements 36-40, Specimen 7-LWLC.... 484

6.237 Shear-Strain Relationship, Vertical Whittemore Measurements 1-2, Specimen 7-LWLC 485

6.238 Shear-Strain Relationship, Vertical Whittemore Measurements 3-4, Specimen 7-LWLC 
6.239 Shear-Strain Relationship, Vertical Whittemore Measurements 5-6, Specimen 7-LWLC

6.240 Shear-Strain Relationship, Vertical Whittemore Measurements 7-8, Specimen 7-LWLC

6.241 Shear-Strain Relationship, Vertical Whittemore Measurements 9-10, Specimen 7-LWLC

6.242 Shear-Strain Relationship, Vertical Whittemore Measurements 11-12, Specimen 7-LWLC

6.243 Shear-Strain Relationship, Vertical Whittemore Measurements 13-14, Specimen 7-LWLC

6.244 Shear-Strain Relationship, Vertical Whittemore Measurements 15-16, Specimen 7-LWLC

6.245 Shear-Strain Relationship, Vertical Whittemore Measurements 17-18, Specimen 7-LWLC

6.246 Shear-Strain Relationship, Diagonal Whittemore Measurements 1-4, Specimen 7-LWLC

6.247 Shear-Strain Relationship, Diagonal Whittemore Measurements 5-8, Specimen 7-LWLC

6.248 Shear-Strain Relationship, Diagonal Whittemore Measurements 9-12, Specimen 7-LWLC

6.249 Shear-Strain Relationship, Diagonal Whittemore Measurements 13-16, Specimen 7-LWLC

6.250 Shear-Strain Relationship, Diagonal Whittemore Measurements 17-20, Specimen 7-LWLC

6.251 Shear-Strain Relationship, Diagonal Whittemore Measurements 21-24, Specimen 7-LWLC 492

6.252 Shear-Strain Relationship, Diagonal Whittemore Measurements 25-28, Specimen 7-LWLC .493

6.253 Shear-Strain Relationship, Diagonal Whittemore Measurements 29-32, Specimen 7-LWLC 
6.254 Shear-Strain Relationship, Horizontal Whittemore Measurements 1-5, Specimen 8-LWLD

6.255 Shear-Strain Relationship, Horizontal Whittemore Measurements 6-10, Specimen 8-LWLD

6.256 Shear-Strain Relationship, Horizontal Whittemore Measurements 11-15, Specimen 8-LWLD

6.257 Shear-Strain Relationship, Horizontal Whittemore Measurements 16-20, Specimen 8-LWLD.

6.258 Shear-Strain Relationship, Horizontal Whittemore Measurements 21-25, Specimen 8-LWLD

6.259 Shear-Strain Relationship, Horizontal Whittemore Measurements 26-30, Specimen 8-LWLD

6.260 Shear-Strain Relationship, Horizontal Whittemore Measurements 31-35, Specimen 8-LWLD

6.261 Shear-Strain Relationship, Horizontal Whittemore Measurements 36-40, Specimen 8-LWLD

6.262 Shear-Strain Relationship, Vertical Whittemore Measurements 1-2, Specimen 8-LWLD

6.263 Shear-Strain Relationship, Vertical Whittemore Measurements 3-4, Specimen 8-LWLD

6.264 Shear-Strain Relationship, Vertical Whittemore Measurements 5-6, Specimen 8-LWLD

6.265 Shear-Strain Relationship, Vertical Whittemore Measurements 7-8, Specimen 8-LWLD

6.266 Shear-Strain Relationship, Vertical Whittemore Measurements 9-10, Specimen 8-LWLD .500

6.267 Shear-Strain Relationship, Vertical Whittemore Measurements 11-12, Specimen 8-LWLD .500

6.268 Shear-Strain Relationship, Vertical Whittemore Measurements 13-14, Specimen 8-LWLD 501 
6.269 Shear-Strain Relationship, Vertical Whittemore Measurements 15-16, Specimen 8-LWLD.

6.270 Shear-Strain Relationship, Vertical Whittemore Measurements 17-18, Specimen 8-LWLD .502

6.271 Shear-Strain Relationship, Diagonal Whittemore Measurements 1-4, Specimen 8-LWLD .503

6.272 Shear-Strain Relationship, Diagonal Whittemore Measurements 5-8, Specimen 8-LWLD .503

6.273 Shear-Strain Relationship, Diagonal Whittemore Measurements 9-12, Specimen 8-LWLD .504

6.274 Shear-Strain Relationship, Diagonal Whittemore Measurements 13-16, Specimen 8-LWLD .504

6.275 Shear-Strain Relationship, Diagonal Whittemore Measurements 17-20, Specimen 8-LWLD 505

6.276 Shear-Strain Relationship, Diagonal Whittemore Measurements 21-24, Specimen 8-LWLD .505

6.277 Shear-Strain Relationship, Diagonal Whittemore Measurements 25-28, Specimen 8-LWLD .506

6.278 Shear-Strain Relationship, Diagonal Whittemore Measurements 29-32, Specimen 8-LWLD .506

6.279 Shear-Strain Relationship, Horizontal Whittemore Measurements 1-5, Specimen 9-NWLD 507

6.280 Shear-Strain Relationship, Horizontal Whittemore Measurements 6-10, Specimen 9-NWLD .507

6.281 Shear-Strain Relationship, Horizontal Whittemore Measurements 11-15, Specimen 9-NWLD .508

6.282 Shear-Strain Relationship, Horizontal Whittemore Measurements 16-20, Specimen 9-NWLD .508

6.283 Shear-Strain Relationship, Horizontal Whittemore Measurements 21-25, Specimen 9-NWLD .509 
6.284 Shear-Strain Relationship, Horizontal Whittemore Measurements 26-30, Specimen 9-NWLD

6.285 Shear-Strain Relationship, Vertical Whittemore Measurements 1-3,

Specimen 9-NWLD

6.286 Shear-Strain Relationship, Vertical Whittemore Measurements 4-6, Specimen 9-NWLD

6.287 Shear-Strain Relationship, Vertical Whittemore Measurements 7-9, Specimen 9-NWLD

6.288 Shear-Strain Relationship, Vertical Whittemore Measurements 10-12, Specimen 9-NWLD

6.289 Shear-Strain Relationship, Vertical Whittemore Measurements 13-15, Specimen 9-NWLD

6.290 Shear-Strain Relationship, Vertical Whittemore Measurements 16-18, Specimen 9-NWLD

6.291 Shear-Strain Relationship, Diagonal Whittemore Measurements 1-6, Specimen 9-NWLD

6.292 Shear-Strain Relationship, Diagonal Whittemore Measurements 7-12, Specimen 9-NWLD

6.293 Shear-Strain Relationship, Diagonal Whittemore Measurements 13-18, Specimen 9-NWLD

6.294 Shear-Strain Relationship, Diagonal Whittemore Measurements 19-24, Specimen 9-NWLD

6.295 Shear-Strain Relationship, Diagonal Whittemore Measurements 25-30, Specimen 9-NWLD

6.296 Shear-Strain Relationship, Diagonal Whittemore Measurements 31-36, Specimen 9-NWLD

6.297 Shear-Strain Relationship, Horizontal Whittemore Measurements 1-5, Specimen 10-NWHD

6.298 Shear-Strain Relationship, Horizontal Whittemore Measurements 6-10, Specimen 10-NWHD 
6.299 Shear-Strain Relationship, Horizontal Whittemore Measurements 11-15, Specimen 10-NWHD.

6.300 Shear-Strain Relationship, Horizontal Whittemore Measurements 16-20, Specimen 10-NWHD.

6.301 Shear-Strain Relationship, Horizontal Whittemore Measurements 2I-25, Specimen 10-NWHD.

6.302 Shear-Strain Relationship, Horizontal Whittemore Measurements 26-30, Specimen 10-NWHD.

6.303 Shear-Strain Relationship, Vertical Whittemore Measurements 1-3, Specimen 10-NWHD.

6.304 Shear-Strain Relationship, Vertical Whittemore Measurements 4-6, Specimen 10-NWHD.

6.305 Shear-Strain Relationship, Vertical Whittemore Measurements 7-9, Specimen 10-NWHD.

6.306 Shear-Strain Relationship, Vertical Whittemore Measurements 10-12, Specimen 10-NWHD.

6.307 Shear-Strain Relationship, Vertical Whittemore Measurements 13-15, Specimen 10-NWHD

6.308 Shear-Strain Relationship, Vertical Whittemore Measurements 16-18, Specimen 10-NWHD.

6.309 Shear-Strain Relationship, Diagonal Whittemore Measurements 1-6, Specimen 10-NWHD

6.310 Shear-Strain Relationship, Diagonal Whittemore Measurements 7-12, Specimen 10-NWHD

6.311 Shear-Strain Relationship, Diagonal Whittemore Measurements 13-18, Specimen 10-NWHD

6.312 Shear-Strain Relationship, Diagonal Whittemore Measurements 19-24, Specimen 10-NWHD

6.313 Shear-Strain Relationship, Diagonal Whittemore Measurements 25-30, Specimen 10-NWHD 
6.314 Shear-Strain Relationship, Diagonal Whittemore Measurements 31-36, Specimen 10-NWHD

6.315 Shear-Strain Relationship, Whittemore Strains, Rosette 1, Specimen 11-LWHD

6.316 Shear-Strain Relationship, Whittemore Strains, Rosette 2, Specimen 11-LWHD

6.317 Shear-Strain Relationship, Whittemore Strains, Rosette 3, Specimen 11-LWHD

6.318 Shear-Strain Relationship, Whittemore Strains, Rosette 4, Specimen 11-LWHD

6.319 Shear-Strain Relationship, Whittemore Strains, Rosette 5, Specimen 11-LWHD

6.320 Shear-Strain Relationship, Whittemore Strains, Rosette 1, Specimen 12-NWHD

6.321 Shear-Strain Relationship, Whittemore Strains, Rosette 2, Specimen 12-NWHD

6.322 Shear-Strain Relationship, Whittemore Strains, Rosette 3, Specimen 12-NWHD

6.323 Shear-Strain Relationship, Whittemore Strains, Rosette 4, Specimen 12-NWHD

6.324 Shear-Strain Relationship, Whittemore Strains, Rosette 5, Specimen 12-NWHD

6.325 Specimen 1-NWLA at Failure .531

6.326 Specimen 1-NWLA at Failure (Part 1 of 3). .531

6.327 Specimen 1-NWLA at Failure (Part 2 of 3).....

6.328 Specimen 1-NWLA at Failure (Part 3 of 3).

6.329 Specimen 2-NWLB at Failure, South End of Test Region

6.330 Specimen 2-NWLB at Failure, East Face, South Central Test Region 
6.331 Specimen 2-NWLB at Failure, East Face, Center of Test Region

6.332 Specimen 2-NWLB at Failure, East Face, North Central Test Region

6.333 Specimen 2-NWLB at Failure, North End of Test Region.

6.334 Specimen 3-NWLC.

6.335 Specimen 3-NWLC.

6.336 Specimen 3-NWLC

6.337 Specimen 3-NWLC

6.338 Specimen 4-NWLD at Failure

6.339 Crushing of North Compression Zone, Specimen 4-NWLD

6.340 Failure Crack Extending into South Support, Specimen 4-NWLD

6.341 Specimen 5-LWLA at Failure (Part 1 of 4)

6.342 Specimen 5-LWLA at Failure (Part 2 of 4).

6.343 Specimen 5-LWLA at Failure (Part 3 of 4).

6.344 Specimen 5-LWLA at Failure (Part 3 of 4)

6.345 Specimen 6-LWLB at Failure (Part 1 of 4)

6.346 Specimen 6-LWLB at Failure (Part 2 of 4)

6.347 Specimen 6-LWLB at Failure (Part 3 of 4)

6.348 Specimen 6-LWLB at Failure (Part 4 of 4)

6.349 Specimen 7-LWLC at Failure (Part 1 of 3)

6.350 Specimen 7-LWLC at Failure (Part 2 of 3)

6.351 Specimen 7-LWLC at Failure (Part 3 of 3)

6.352 Specimen 8-LWLD at Failure

6.353 Specimen 9-NWLD at Failure (Part 1 of 4) 
6.354 Specimen 9-NWLD at Failure (Part 2 of 4)

6.355 Specimen 9-NWLD at Failure (Part 3 of 4)

6.356 Specimen 9-NWLD at Failure (Part 4 of 4)

6.357 Specimen 10-NWHD at Failure

6.358 Specimen 10-NWHD at Failure (Part 1 of 2)

6.359 Specimen 10-NWHD at Failure (Part 2 of 2)

6.360 Specimen 11-LWHD at Failure

6.361 Specimen 11-LWHD at Failure (South Test Region)

6.362 Specimen 11-LWHD at Failure (North Test Region)

6.363 Specimen 12-NWHD at Failure

6.364 Specimen 12-NWHD at Failure (South Test Region)

6.365 Specimen 12-NWHD at Failure (North Test Region)

6.366 Shear-Deflection Relationship for Specimen PC@N .550

6.367 Shear-Longitudinal Steel Strain Relationship for Specimen PC@N .550

6.368 Shear-Strand Strain Relationship for Specimen PC๑N........................................551

6.369 Shear-Deflection Relationship for Specimen PC6S …………...........................552

6.370 Shear-Longitudinal Steel Strain Relationship for Specimen PC6S .....................552

6.371 Shear-Strand Strain Relationship for Specimen PC6S ......................................553

6.372 Shear-Stirrup Strain Relationship for Specimen PC6S, Stirrups T1-T5 ...............553

6.373 Shear-Stirrup Strain Relationship for Specimen PC6S, Stirrups T6-T10 .............554

6.374 Shear-Deflection Relationship for Specimen PC10N .......................................555

6.375 Shear-Longitudinal Steel Strain Relationship for Specimen PC10N ..................555

6.376 Shear-Deflection Relationship for Specimen PC10S .....................................556 
6.377 Shear-Longitudinal Steel Strain Relationship for Specimen PC10S

6.378 Shear-Stirrup Strain Relationship for Specimen PC10S, Stirrups T1-T5 …........557

6.379 Shear-Stirrup Strain Relationship for Specimen PC10S, Stirrups T6-T10 ..........557

6.380 Shear-Strain Relationship, Whittemore Strains, Rosette 1, Specimen PC6N......558

6.381 Shear-Strain Relationship, Whittemore Strains, Rosette 2, Specimen PC6N......558

6.382 Shear-Strain Relationship, Whittemore Strains, Rosette 3, Specimen PC6N......559

6.383 Shear-Strain Relationship, Whittemore Strains, Rosette 1, Specimen PC6S ......560

6.384 Shear-Strain Relationship, Whittemore Strains, Rosette 2, Specimen PC6S ......560

6.385 Shear-Strain Relationship, Whittemore Strains, Rosette 3, Specimen PC6S ......561

6.386 Shear-Strain Relationship, Whittemore Strains, Rosette 1, Specimen PC10N....562

6.387 Shear-Strain Relationship, Whittemore Strains, Rosette 2, Specimen PC10N....562

6.388 Shear-Strain Relationship, Whittemore Strains, Rosette 3, Specimen PC10N....563

6.389 Shear-Strain Relationship, Whittemore Strains, Rosette 4, Specimen PC10N....563

6.390 Shear-Strain Relationship, Whittemore Strains, Rosette 5, Specimen PC10N....564

6.391 Shear-Strain Relationship, Whittemore Strains, Rosette 1, Specimen PC10S ....565

6.392 Shear-Strain Relationship, Whittemore Strains, Rosette 2, Specimen PC10S ....565

6.393 Shear-Strain Relationship, Whittemore Strains, Rosette 3, Specimen PC10S ....566

6.394 Shear-Strain Relationship, Whittemore Strains, Rosette 4, Specimen PC10S ....566

6.395 Shear-Strain Relationship, Whittemore Strains, Rosette 5, Specimen PC10S ....567

6.396 Failure Crack in North Shear Span, Specimen PC6 N.........................................568

6.397 Spalling of Web in North Shear Span, Specimen PC@ ....................................568

6.398 Failure Crack Extending to Face of Support, Specimen PC $₫$............................569

6.399 Web-Shear Crack in South Shear Span at Failure, Specimen PC6N....................569 
6.400 Failure Crack in South Shear Span, Specimen PC6S

6.401 Crushing of Top Flange, Specimen PC6S .570

6.402 Splitting Crack Along Prestressing Reinforcement, Specimen PC6S

6.403 Web-Shear Crack in North Shear Span, Specimen PC6S.

6.404 Major Web-Shear Crack in North Shear Span, Specimen PC10N

6.405 Crushing of Upper Flange near Midspan, Specimen PC10N

6.406 Web-Shear Crack in North Shear Span, Specimen PC10N.

6.407 Undamaged Transfer Length Zone Behind Support, Specimen PC10N

6.408 Web-Shear Crack in South Shear Span, Specimen PC10S, East Side.

6.409 Web-Shear Crack in South Shear Span, Specimen PC10S, West Side

6.410 Web-Shear Cracks in North Shear Span, Specimen PC10S

6.411 Crushing of Upper Web at Flange near Midspan, Specimen PC10S

6.412 Ruptured Stirrups after Removal of Web Concrete, Specimen PC10S

6.413 Locations of Critical Sections for Shear, Reinforced Concrete Specimens .577

6.414 Comparison of Measured Shear Capacity and Code-Estimated Shear Capacities, Specimen 1-NWLA

6.415 Comparison of Measured Shear Capacity and Code-Estimated Shear Capacities, Specimen 2-NWLB.

6.416 Comparison of Measured Shear Capacity and Code-Estimated Shear Capacities, Specimen 3-NWLC.

6.417 Comparison of Measured Shear Capacity and Code-Estimated Shear Capacities, Specimen 4-NWLD

6.418 Comparison of Measured Shear Capacity and Code-Estimated Shear Capacities, Specimen 5-LWLA. .580

6.419 Comparison of Measured Shear Capacity and Code-Estimated Shear Capacities, Specimen 6-LWLB .580 
6.420 Comparison of Measured Shear Capacity and Code-Estimated Shear Capacities, Specimen 7-LWLC

6.421 Comparison of Measured Shear Capacity and Code-Estimated Shear Capacities, Specimen 8-LWLD

6.422 Comparison of Measured Shear Capacity and Code-Estimated Shear Capacities, Specimen 9-NWLD

6.423 Comparison of Measured Shear Capacity and Code-Estimated Shear Capacities, Specimen 10-NWHD.

6.424 Comparison of Measured Shear Capacity and Code-Estimated Shear Capacities, Specimen 11-LWHD 583

6.425 Comparison of Measured Shear Capacity and Code-Estimated Shear Capacities, Specimen 12-NWHD. .583

6.426 Locations of Critical Sections for Shear, Prestressed Concrete Specimens 584

6.427 Comparison of Measured Shear Capacity and Code-Estimated Shear Capacities, Specimen PC6N

6.428 Comparison of Measured Shear Capacity and Code-Estimated Shear Capacities, Specimen PC6S.

6.429 Comparison of Measured Shear Capacity and Code-Estimated Shear Capacities, Specimen PC10N 586

6.430 Comparison of Measured Shear Capacity and Code-Estimated Shear Capacities, Specimen PC10S. .586

B.1 Free Body Diagram of Beam (AASHTO LRFD Method). .616 


\section{NOTATION}

The notation used throughout this report is summarized in this section. The notation is organized according to the section in which appears. Some notation is repeated if it appears in multiple sections in the report.

\subsection{Properties of Lightweight Concrete}

$E_{c}=$ modulus of elasticity of concrete

$\mathrm{f}_{\mathrm{c}}=\quad$ concrete compressive strength of $100 \times 200 \mathrm{~mm}$ cylinders

$\mathrm{f}_{\mathrm{c}}=$ standard concrete cylinder strength

$\rho \quad=\quad$ density of concrete

\subsection{Shear Strength of Concrete Beams}

a $=$ distance from the concentrated load to the support

$\mathrm{d}=$ depth of the tensile reinforcement

$\mathrm{M}=$ moment at the section

$\mathrm{V}=$ shear force at the section

$\mathrm{V}_{\mathrm{cz}}=$ shear force carried by the shear stress in the uncracked compression zone

$\mathrm{V}_{\mathrm{ay}}=$ shear force carried by aggregate interlock

$\mathrm{V}_{\mathrm{d}}=$ shear force carried by dowel action

$\mathrm{V}_{\mathrm{s}}=$ shear force carried by shear reinforcement 


\subsection{Shear Tests}

$\mathrm{f}_{\mathrm{c}}=$ compressive strength of equivalent standard concrete cylinder

$\mathrm{f}_{\mathrm{c}}{ }_{\mathrm{c}}=$ standard concrete cylinder strength

$\mathrm{f}_{\mathrm{ct}}=\quad$ split cylinder strength of concrete

$\mathrm{f}_{\mathrm{sy}} \quad=\quad$ yield stress of shear reinforcement

\subsubsection{AASHTO LRFD}

$\mathrm{A}_{\mathrm{c}}=$ area of concrete on the flexural tension side of the member

$\mathrm{A}_{\mathrm{s}}=$ area of non-prestressed reinforcing steel on the flexural tension side of the member, reduced for any lack of full development at the section under investigation

$\mathrm{A}_{s}=$ area of prestressing steel on the flexural tension side of the member, reduced for any lack of full development at the section under investigation

$\mathrm{A}_{\mathrm{v}}=$ area of shear reinforcement within a distance $\mathrm{s}$

$b_{v}=\quad$ effective web width taken as the minimum web width within the depth $d_{v}$

$\mathrm{d}_{\mathrm{e}}=$ effective depth from extreme compression fiber to the centroid of the tensile force in the tensile reinforcement

$\mathrm{d}_{\mathrm{v}}=\quad$ effective shear depth taken as the distance, measured perpendicular to the neutral axis, between the resultants of the tensile and compressive forces due to flexure, but it need not be taken less than the greater of $0.9 \mathrm{~d}_{\mathrm{e}}$ or $0.72 \mathrm{~h}$

$\mathrm{E}_{\mathrm{c}}=$ modulus of elasticity of concrete

$\mathrm{E}_{\mathrm{p}} \quad=\quad$ modulus of elasticity of prestressing tendons

$\mathrm{E}_{\mathrm{s}} \quad=\quad$ modulus of elasticity of reinforcing bars

$\mathrm{f}_{\mathrm{c}}{ }_{\mathrm{c}}=$ specified compressive strength of concrete at 28 days, unless another age is specified

$\mathrm{F}_{\varepsilon}=$ reduction factor that accounts for the stiffness of the concrete in 
compression

$\mathrm{f}_{\mathrm{y}} \quad=\quad$ specified minimum yield strength of reinforcing bars

$\mathrm{h}=\quad$ overall thickness or depth of member

$\mathrm{M}_{\mathrm{u}} \quad=\quad$ factored moment at the section

$\mathrm{S} \quad=\quad$ spacing of the shear reinforcement

$\mathrm{s}_{\mathrm{X}} \quad=\quad$ crack spacing parameter

$\mathrm{V}_{\mathrm{c}}=$ nominal shear resistance provided by tensile stresses in the concrete

$\mathrm{V}_{\mathrm{n}}=$ nominal shear resistance of the section considered

$\mathrm{V}_{\mathrm{p}}=$ component in the direction of the applied shear of the effective prestressing force, positive if resisting the applied shear

$\mathrm{V}_{\mathrm{S}} \quad=\quad$ shear resistance provided by shear reinforcement

$\mathrm{V}_{\mathrm{u}}=$ factored shear force at section

$\beta=$ factor relating effect of longitudinal strain on the shear capacity of concrete, as indicated by the ability of diagonally cracked concrete to transmit tension

$\varepsilon_{\mathrm{x}}=$ longitudinal strain in the reinforcement on the flexural tension side of the member

$\theta=$ angle of inclination of diagonal compressive stresses

$\mathrm{v}=$ factored shear stress in the concrete

\subsubsection{ACI 318-95/AASHTO}

$\mathrm{A}_{\mathrm{S}} \quad=\quad$ area of non-prestressed tension reinforcement

$\mathrm{A}_{\mathrm{v}} \quad=\quad$ area of shear reinforcement within a distance $\mathrm{s}$

$b_{w} \quad=\quad$ web width

$\mathrm{d}=$ distance from extreme compression fiber to centroid of longitudinal 
tension reinforcement, but need not be less than $0.80 \mathrm{~h}$ for prestressed members

$\mathrm{f}_{\mathrm{ct}} \quad=\quad$ average splitting tensile strength of lightweight aggregate concrete
$\mathrm{f}_{\mathrm{pc}} \quad=\quad$ compressive stress in concrete (after allowance for all prestress losses) at centroid of cross section resisting externally applied loads at junction of web and flange when the centroid lies within the flange. In a composite member, $f_{p c}$ is resultant compressive stress at centroid of composite section, or at junction of web and flange, due to both prestress and moments resisted by precast member acting alone

$\mathrm{f}_{\mathrm{y}}=$ specified yield strength of nonprestressed reinforcement

$\mathrm{h}=$ overall thickness of member

$\mathrm{M}_{\mathrm{cr}} \quad=\quad$ moment causing flexural cracking at section due to externally applied loads

$\mathrm{M}_{\max }=\quad$ maximum factored moment at section due to externally applied loads

$\mathrm{M}_{\mathrm{u}}=$ factored moment at section

$\mathrm{s}=\quad$ spacing of shear reinforcement in direction parallel to longitudinal reinforcement

$\mathrm{V}_{\mathrm{c}}=$ nominal shear strength provided by concrete

$\mathrm{V}_{\mathrm{ci}}=$ nominal shear strength provided by concrete when diagonal cracking results from combined shear and moment

$\mathrm{V}_{\mathrm{cw}}=$ nominal shear strength provided by concrete when diagonal cracking results from excessive principal tensile stress in web

$\mathrm{V}_{\mathrm{d}}=$ shear force at section due to unfactored dead load

$\mathrm{V}_{\mathrm{i}}=$ factored shear force at section due to externally applied loads occurring simultaneously with $\mathrm{M}_{\max }$

$\mathrm{V}_{\mathrm{n}}=$ nominal shear strength

$\mathrm{V}_{\mathrm{p}}=$ vertical component of effective prestress force at section

$\mathrm{V}_{\mathrm{s}}=$ nominal shear strength provided by the shear reinforcement 
$\mathrm{V}_{\mathrm{u}} \quad=\quad$ factored shear force at the section

$\rho_{\mathrm{w}} \quad=\quad \mathrm{A}_{\mathrm{s}} / \mathrm{b}_{\mathrm{w}} \mathrm{d}$

xlix 


\section{CHAPTER 1. INTRODUCTION}

\section{$\underline{1.1 \text { General Background }}$}

Structural engineers have continually tried to improve the quality and effectiveness of their building materials. Normal-strength lightweight aggregate concrete (NSLWC) has been used in place of normal-strength normal-weight concrete (NSNWC) in building applications to decrease dead load and reduce member dimensions. A notable example includes the following: The Australian Square, Sydney, Australia, completed in 1967 , is a circular tower (50 stories) $184 \mathrm{~m}$ high by $42.5 \mathrm{~m}$ in diameter. A $13 \%$ saving in construction costs was achieved using $31,000 \mathrm{~m}^{3}$ of lightweight aggregate concrete in the beams, columns, and floors above the seventh-floor level. The concrete had an average compressive strength of $34.3 \mathrm{MPa}$ and an average density of $1792 \mathrm{~kg} / \mathrm{m}^{3}$ at 28 days.

Ligthweight aggregate concrete has been used in American highway bridges for over 50 years and there are more than 200 concrete and composite bridges containing lightweight aggregates in the United States and Canada [Raithby and Lydon, 1981]. In the former USSR about 100 bridges have been constructed using lightweight aggregates in the past 20 years and in Europe the numbers are increasing steadily.

It is in bridge construction that lightweight aggregate has made some of its most significant contributions to construction efficiency. LWC has been successfully used in applications ranging from simple reinforced concrete footbridges to long span post tension segmental box girder bridges. Weight savings of $30 \%$ on the superstructure can be achieved in some cases, with consequent savings of reinforcing and prestressing steel. The size of the piers and foundations can also be reduced. Overall savings on cost of 
more than $10 \%$ can be expected after allowances have been made for the higher initial cost of lightweight aggregates.

The versatility of lightweight aggregate is such that it lends itself well to be used in both precast and cast-in-place applications. This facilitates the efficient coordination of the different elements of a job and makes for improved construction schedules and lower costs. In the 1980 s more than 50 prestressed concrete cantilever bridges have been built with main span length in the range of $123-183 \mathrm{~m}$ (400-660 ft) using normal weight aggregates. The utilization of high strength concrete using a combination of normal weight aggregates (NWA) and LWA offers new possibilities in extending the span length of these cantilever bridges in an economic way.

LWC bridges have generally performed quite satisfactorily in service and there is evidence that their durability is comparable or even better than normal weight concrete [ESCSI, ACI 1991]. The few reported cases of unsatisfactory performance may have been due to inadequate detail design or poor quality control during construction [Raithby and Lydon, 1981]. There is evidence that in practice the creep and shrinkage of LWC are much smaller than obtained from small-scale laboratory tests [Raithby and Lydon, 1981]. Creep and shrinkage measurements in some of the bridges have indicated results close to those of normal weight concrete. It has been reported that shrinkage cracking has been eliminated and frost resistance and seismic stability improved [Raithby and Lydon, 1981]. Similar results to normal weight concrete were obtained for dynamic response to moving traffic [Raithby and Lydon, 1981].

LWC is particularly advantageous in prestressed and precast components to reduce handling costs where access is limited or where ground conditions are severe. For 
this reason LWC can be particularly well suited for such applications as upgrading of existing bridge structures to improvements to local roads where disruption to traffic must be kept at a minimum.

\subsection{Outline of Report}

In this report, Chapter 2 contains an extensive literature review of the material characteristics and mix design of LWC, the shear transfer mechanisms, information on lightweight aggregate concrete, a survey of relevant research on the shear strength of reinforced and prestressed concrete beams, and an explanation of US code procedures to estimate the shear strength of reinforced and prestressed concrete beams. Chapter 3 gives the problem statement, research objectives and the scope of research. Chapter 4 contains a description of the work plan including material properties, mixture proportioning, specimen details, instrumentation, and fabrication procedures. The description of the experimental program is contained in Chapter 5. Chapter 6 contains the results obtained during the testing program and the analysis of the experimental behavior. Chapter 7 contains the summary of significant findings, conclusions from the study, and lists recommendations based on the results of the research. The appendices contain design tables from the AASHTO LRFD and derivations of equations used in the development of the AASHTO LRFD.

\subsection{Terminology Used in the Report}

The following is a list of the terms used in this thesis:

LWA Lightweight aggregate

NWA Normal weight aggregate

S

Silica Fume 


\section{F}

$\mathrm{H}$

$\mathrm{MI}$

L

G

LWC

NWC

NWC-Plant

SM-LWC

LWCH

LWCHS

LWCHS-Plant

LWCHF

LWCH-REF
Fly Ash

Haydite lightweight aggregate

Minergy lightweight aggregate

Crushed limestone aggregate

River gravel

Lightweight aggregate concrete. Concrete made with lightweight coarse aggregate and natural sand.

Normal weight concrete. Concrete made with normal weight aggregates.

Normal weight concrete. Concrete made with normal weight aggregates. Concrete produced at the plant.

Semi-lightweight concrete. Concrete made a blend of normal weight coarse aggregates and lightweight coarse aggregates and natural sand.

Lightweight aggregate concrete with Haydite lightweight aggregates.

Lightweight aggregate concrete with Haydite lightweight aggregate and silica fume.

Lightweight aggregate concrete with Haydite lightweight aggregate and silica fume. Concrete produced at the plant. Lightweight aggregate concrete with Haydite lightweight aggregate and fly ash.

Reference lightweight aggregate concrete with Haydite lightweight aggregate. 
LWCHS-REF

LWCHF-REF

NWC-REF

LWCMI

LWCMIS

SM-LWCHL

SM-LWCHLS

HRWR

WR

RET

AEA

ESCI

W/C

W/CEM

NSLWC

NSNWC
Reference lightweight aggregate concrete with Haydite lightweight aggregate and silica fume.

Reference lightweight aggregate concrete with Haydite lightweight aggregate and fly ash.

Reference normal weight concrete.

Lightweight aggregate concrete with Minergy lightweight aggregate

Lightweight aggregate concrete with Minergy lightweight aggregate and silica fume

Semi-LWC with the Haydite lightweight aggregate and limestone normal weight aggregate.

Semi-LWC with the limestone normal weight aggregate, Haydite lightweight aggregate and silica fume.

High range water reducer admixture.

Water reducer admixture.

Retarder admixture.

Air entraining agent admixture.

Expanded Shale and Clay Institute

Water-to-cement

Water-to-cementitious

Normal Strength Lightweight Conference

Normal Strength Normal Weight Concrete 


\section{CHAPTER 2. LITERATURE REVIEW}

\subsection{Introduction}

This chapter contains an overview of the following items:

- Characteristics of lightweight aggregates

- Structural Lightweight Concrete in Bridge Construction

- Physical and Mechanical Properties of lightweight concrete

- Durability of Lightweight Concrete

- Mix design of structural lightweight concrete

- Shear strength of concrete beams

- Beam shear tests

- U.S. Code and Specifications methods of calculating the shear strength

The section dedicated to the shear strength of concrete beams contains an explanation of the mechanisms of shear resistance in reinforced and prestressed concrete beams. The sections on lightweight aggregate concrete describe characteristic sources, properties of lightweight aggregates and lightweight aggregate concrete and mix design. The section on the beam shear tests contains experimental studies relevant to this experimental study. Lastly, code and specification approaches for determining the shear strength of reinforced and prestressed beams with normal weight and lightweight aggregate concrete are described. 


\subsection{Characteristics of Lightweight Aggregates}

The term lightweight aggregate describes a range of special aggregates that have specific gravities considerably below normal sand and gravel [ACI 1991, Holm 19??]. These lightweight aggregates range from the extremely light materials used for insulative and non-structural concrete all the way to expanded clays and shales used for structural concrete. The lightness of these aggregates is derived from the air trapped in each particle and the more air that is trapped per particle unit, the lighter the weight and the better the insulation, but conversely, the strength is lower.

The cellular pore structure within the particles of most lightweight aggregates is developed as a result of formation of gases during the process of decomposition at high temperatures. Durable and strong lightweight aggregates are formed when small, well distributed, non-interconnected pores are enveloped in a continuous, crack free, vitreous phase.

The unit weight of lightweight aggregates varies depending on the source and type of aggregate. Lightweight aggregates exhibit considerable differences in particle shape and texture depending on the source and method of production. Shapes may be cubical, rounded, angular, or irregular. Textures may range from highly irregular surfaces with large exposed pores to smooth surfaces with fine pores. The specific gravity of the dry LWA is significantly lower than that of conventional aggregates, ranging from 1.1 to 2.2 . Structural concrete made with these aggregates is usually 20 to 30 percent lighter than conventional concrete.

Gradation requirements of LWA are generally similar to those provided for NWA with the exception that lightweight aggregate particle size distribution permits higher 
weight through smaller sieves [Holm 19??]. This modification recognizes the increase in specific gravity that is typical for smaller particles of most lightweight aggregates, and that while standards are established by weights passing each sieve, the ideal formulations are developed through volumetric considerations [Holm 19??].

Producers of structural lightweight aggregate normally stock material in several standard size formulations of coarse, intermediate, and fine aggregate. A wide range of unit weights can be achieved by combining size fractions or by replacing some or all of the fine fraction with normal weight sand. Information can be readily obtained from the aggregate producers with regards to the proper aggregate combinations to meet fresh unit weight specifications and equilibrium unit weights for dead load design consideration.

The cellular microstructure of lightweight aggregates renders them more absorbent to water than normal weight aggregates [Holm 19??, ACI 1987]. The 24-hour absorption of lightweight aggregates can vary from $5 \%$ to $25 \%$ by weight of dry aggregate. NWA usually absorb less than $2 \%$ of moisture. In LWA the moisture is largely absorbed into the interior of the particles whereas, in NWA it is primarily absorbed on the surface. This characteristic is important for lightweight aggregate concrete mixture proportioning, batching, and quality control. Rate of absorption for LWA is dependent on the characteristics of size, connectivity and distribution of pores, particularly those close to the surface. Internally absorbed water within the particles is not immediately available for chemical interaction with the cement as mixing water, but is extremely beneficial over time by providing additional curing essential to improvements to the aggregate-matrix interfacial zone. Internal curing will also bring 
about reduction of permeability by extending the period in which additional products of hydration are formed in the pores and capillaries of the binder.

\subsection{Structural Lightweight Concrete in Bridge Construction}

The use of lightweight aggregate concrete in bridge construction is rapidly increasing. With increasing use of high strength concrete, it became possible to increase the span length of bridges up to $201 \mathrm{~m}$ (660 ft) [Fergestad et al., 1988, Roberts 1997]. As span length increases, the amount of materials required increases rapidly, and the selfweight of the structure becomes the dominant load for these bridges. The utilization of high strength concrete with the combination of NWA and LWA offers new possibilities in extending the span length of bridges in an economical way $[17,18,19,20,21]$. Compared to NWC the initial cost of LWC may be higher due to higher cost of high quality LWA and larger amount of cement required to achieve high strength. High cement contents may also present the problem of thermal cracking. Stricter field quality control of moisture content of the lightweight aggregate is required.

The Expanded Shale, Clay and Slate Institute (ESCI) reported in 1953 that there were 40 bridges in North America in which lightweight concrete had been used in the main structure [Raithby and Lydon, 1981]. That number had risen to more than 150 by 1960 and now may well be past the 200 figure. Most of these bridges are in the USA, while some are in Canada. The use of LWC in highway bridges in Europe has been more recent within the last 15 years [Raithby and Lydon, 1981]. Very few LWC bridges have been built in England, but there appears to be potential advantages for more extensive use 
of LWC in bridge construction. In Russia, Germany, and Poland there is increasing use of LWC in the construction of bridges.

Expanded shale (Haydite) was used in the 1920s to build the first lightweight concrete bridges. The experienced gained on these early bridges, led to more adventurous uses, and taking advantage of the low density led to more significant savings in construction costs. There were a number of successful applications of LWC in bridge construction reported during this period and this led to usage of lightweight concrete in the upper deck of the San Francisco-Oakland Suspension Bridge in the 1930s [Raithby and Lydon, 1981]. The use of LWC on this bridge resulted in savings of $\$ 3$ million in construction costs. LWC was used again when this bridge was modernized, and more recently, has been used for redecking the Golden Gate Bridge.

In the construction of the bridge across Sebastian Inlet on the east coast of Florida, three conditions were imposed on the designers.

1. The structure had to be unaffected by the corrosive action of the salt air.

2. The channel had to be kept open during the construction, and could not be constricted by falsework.

3. The main span had to be $55 \mathrm{~m}$ (180 ft) long to comply with Corps of Engineers guidelines.

The $55 \mathrm{~m}$ (180 ft) long main span was achieved by using $37 \mathrm{~m}$ (120 ft) drop-in girders of structural lightweight concrete supported by anchor-cantilever girders of

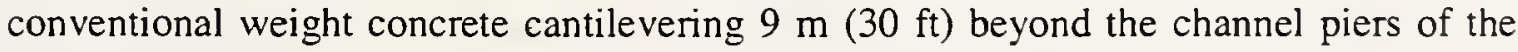
bridge. Structural LWC was used for the top slabs and superstructure of pontoons at the floating bridge across Lake Washington in Seattle. This reduced their displacement 
which was central to a design that resulted in a cost of 24 million, as contrasted with the

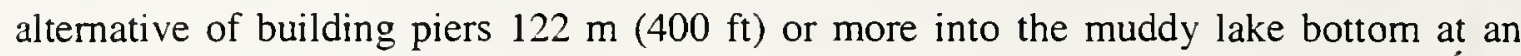
estimated cost of $\$ 50$ million.

A single-span precast lightweight concrete bridge stretching over the southern Washington's Klickitat River was built with its $40 \mathrm{~m}$ (131 ft) span consisting of four beams set side by side, each $1 \mathrm{~m}$ wide and $2 \mathrm{~m}$ deep ( $4 \mathrm{ft}$ wide and $4 \mathrm{ft} 11 \mathrm{in}$. deep) and weighing $47,627 \mathrm{~kg}$ (105,000 lbs.), yielding a weight savings of $16,783 \mathrm{~kg}(37,000 \mathrm{lbs}$. per beam over a sand and gravel concrete beam. The bridge, completed in 1965, represented a great stride forward in slightly more than a decade from a footbridge erected in Prairie Village, Kansas in 1954 with beams measuring $16 \mathrm{~m} \mathrm{(52} \mathrm{ft)} \mathrm{long.}$

Confidence in the durability of LWC in maritime environment was reflected in the construction in 1975 of a second lightweight concrete bridge alongside the original Chesapeake Bay Bridge, which was built of LWC in 1952 [Raithby and Lydon, 1981]. Upon removal of the asphalt surface of the original bridge in 1975 , it was found that the lightweight concrete deck of the main span was in a much better condition than the ordinary concrete in the approach spans.

Lightweight concrete was used in post-tensioned segmental box girder, $691 \mathrm{~m}$ (2270 ft) long Napa River bridge which was completed in 1975. The cost was \$11 million compared to $\$ 16.7$ million for an alternative steel box girder design. The Parrots Ferry Road Bridge has spans of $201 \mathrm{~m}$ (660 ft) and was constructed at a cost of $\$ 10.5$ million. This bridge has a $12 \mathrm{~m}(40 \mathrm{ft})$ wide deck carried by a box about $7 \mathrm{~m}(23 \mathrm{ft})$

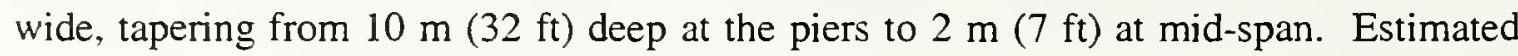


cost savings of $10 \%$ and $20 \%$ weight reduction were achieved using air-entrained lightweight concrete.

In Russia, between 1961 and 1978, about 100 bridges were constructed using lightweight concrete. The majority of these bridges were prestressed girders with spans of up to $34 \mathrm{~m}$ (110 ft). The type of aggregate usually used in Russia and Eastern Europe is expanded clay. The most recent application makes use of partially prestressed lightweight concrete in parts of the structure where it can be most effective, in combination with high grade normal weight concrete in other parts of the structure [Raithby and Lydon, 1981]. Claims of 25 to $30 \%$ weight savings have been made with a reduction of 15 to $18 \%$ in the amount of reinforcing and prestressing steel.

Using LWC in suspended structures using lightweight concrete with spans exceeding $201 \mathrm{~m}(660 \mathrm{ft})$, reduced design loads by more than $30 \%$, with a saving of more than $15 \%$ on prestressing steel. The greatest cost saving has been achieved using lightweight concrete in bridge spans exceeding $40 \mathrm{~m}(133 \mathrm{ft})$.

In Poland recent development has been in the construction and testing of a composite bridge having precast prestressed lightweight I-beams with an in-situ lightweight deck slab [Raithby and Lydon, 1981]. The aggregate used was expanded shale. Full-scale laboratory tests have been carried out to check ultimate load capacity, shrinkage, creep and deflection. Loading tests were carried out on the completed bridge to check deflections under heavy vehicles. The lightweight spans had approximately twice the deflections of the normal weight concrete.

In West Germany, one of the earliest bridges made using lightweight concrete was a footbridge at Wiesbaden that was constructed in 1967 [Raithby and Lydon, 1981]. 
It is an arch bridge of $155 \mathrm{~m}(510 \mathrm{ft})$ total length with cantilevered approach ramps forming structural counter-balance to the $94 \mathrm{~m}(310 \mathrm{ft})$ main arch. The aggregate used was expanded shale.

Between 1968 and 1971, many other bridges with lightweight concrete in the main structure were constructed in Germany. The $86 \mathrm{~m}$ (283 ft) span bridge crossing a motorway near Osnabruck was built as two cantilevers connected by a shear-transmitting joint at midspan. The abutments were made of normal weight concrete and acted as counter-weights to the cantilever section. The cantilever section was made using a lightweight concrete post-tensioned box of variable depth.

Holland, Belgium, and France have all had experience with lightweight concrete bridge construction. However, two particularly interesting bridges have recently been completed in France [Raithby and Lydon, 1981]. One is the Alsace Canal Bridge at Ottmarshein that carries an A36 autoroute and has two spans. The central portion (101 $\mathrm{m} / 333 \mathrm{ft})$ of the longer span $(175 \mathrm{~m} / 573 \mathrm{ft})$ is made out of LWC. This unique bridge is a twin concrete box of post-tensioned segmental construction. The use of lightweight concrete resulted in a saving of weight and enabled the length of the relevant segments to be increased from $2 \mathrm{~m}$ to $3 \mathrm{~m} \mathrm{(} 7 \mathrm{ft}$ to $10 \mathrm{ft}$ ). This reduced the number of joints required. This hybrid design was chosen as the most economical among the alternative designs.

In the construction of the Tricastin Bridge the use of lightweight concrete showed distinct advantages. Lightweight concrete was used for the central portion $(109 \mathrm{~m} / 356 \mathrm{ft})$ of the $144 \mathrm{~m}(473 \mathrm{ft})$ span. The structure is a prestressed box and the use of lightweight concrete reduced the maximum bending moment by about $25 \%$ when compared to NWC. The negative bending moments were significantly reduced as a result of the smaller self 
weight, smaller counter weight required on the short spans and reduced loads on the bridge bearings, resulting in cost savings on the foundations.

Finding increasing use in prefabricated bridges is a new type of LWC called semilightweight concrete. This type of LWC is similar to NWC but has about $20 \%$ of the medium sized aggregate replaced by a lightweight aggregate resulting in an average density of $2000 \mathrm{~kg} / \mathrm{m}^{3}$ ( $135 \mathrm{pcf}$ ). This reduces the design weight by 10 to $15 \%$ and the modulus of elasticity by roughly $25 \%$.

Because structural LWC is typically $25-30 \%$ lighter, it requires less reinforcing, prestressing and structural steel. It also increases live load capacity, permits longer spans, and permits deeper sections while maintaining the same dead load. The use of lightweight aggregate concrete allows for bridge upgrades and expansions without replacing or adding support foundations.

Lightweight aggregate concrete exhibits high durability characteristics. Some of these properties are: low permeability, high freeze/thaw resistance, and high resistance to deicing salts and chemicals. Elastic compatibility between aggregate and matrix reduces the internal stresses and minimizes microcracking and ensures superior bond between the aggregate and the matrix [Caltrans and Pacific Custom Materials, 1996]. Resistance of LWA to polishing under traffic ensures a high skid resistant surface and thus improves roadway safety.

Lightweight aggregate concrete allows greater design flexibility to meet today's challenges of design and construction. High compressive strengths can be achieved to meet modern engineering requirements. Structural LWC has a proven performance record of successful use in severely exposed marine bridge construction for more than 70 
years. It has been subjected to extreme weather and loading conditions, and has proven sound and very durable.

\subsection{Physical and Mechanical Properties of Lightweight Concrete}

This section contains general description of properties of lightweight concrete including density, strength, and modulus of elasticity.

The practical range of densities of lightweight aggregate concrete is between 300 and $1900 \mathrm{~kg} / \mathrm{m}^{3}$. Structural lightweight concrete typically has a density between 1350 and $1900 \mathrm{~kg} / \mathrm{m}^{3}$ and a minimum compressive strength of $17 \mathrm{MPa}$. Lightweight aggregates (LWA) are produced from a variety of raw materials and production methods. Consequently, the properties of LWA may vary within wide limits (Zhang and Gjorv, 1991).

For a given lightweight aggregate, there exists a broad relation between the cement content of concrete and its compressive strength. The amount of cement needed for a $40 \mathrm{MPa}$ mix may be between $420 \mathrm{~kg} / \mathrm{m}^{3}$ and $500 \mathrm{~kg} / \mathrm{m}^{3}$. The amount of cement needed for a $70 \mathrm{MPa}$ mix may be $630 \mathrm{~kg} / \mathrm{m}^{3}$. In this study, $422 \mathrm{~kg} / \mathrm{m}^{3}$ of cement were used to achieve a compressive strength of $43 \mathrm{MPa}$ in the lightweight aggregate mix. For the $72 \mathrm{MPa}$ mix, $621 \mathrm{~kg} / \mathrm{m}^{3}$ of cementitious material was used $\left(565 \mathrm{~kg} / \mathrm{m}^{3}\right.$ of cement and $56 \mathrm{~kg} / \mathrm{m}^{3}$ of silica fume). As in normal weight concrete, silica fume improves the strength development of lightweight aggregate concrete. Other cementitious materials, including fly ash, can also be incorporated in lightweight aggregate concrete. It has been observed that with the addition of condense silica fume to a $69 \mathrm{MPa}$ lightweight 
aggregate concrete, the steel-concrete bond strength doubled compared to NWC and LWC of the same compressive strength without silica fume (Mor, 1992).

In general terms, for the same compressive strength of concrete, the cement content in a lightweight aggregate mix is higher than in normal weight concrete; at high strengths, the additional cement content can exceed 50 percent. In this study, $303 \mathrm{~kg} / \mathrm{m}^{3}$ of cement used for a $46 \mathrm{MPa}$ normal weight mix compared with $422 \mathrm{~kg} / \mathrm{m}^{3}$ of cement used in the $43 \mathrm{MPa}$ lightweight aggregate mix. For the $72 \mathrm{MPa}$ lightweight aggregate $\operatorname{mix}, 621 \mathrm{~kg} / \mathrm{m}^{3}$ of cementitious material was used compared with $463 \mathrm{~kg} / \mathrm{m}^{3}$ of cementitious material used in the $75 \mathrm{MPa}$ normal weight mix.

Because of the high cement content of lightweight aggregate concrete, the strength of the matrix is high. Combined with the fact that lightweight particles of coarse aggregate are relatively weak, their strength may be the limiting factor in the strength of the concrete. The limitation on the strength of lightweight aggregate concrete imposed by the strength of the coarse aggregate particles can be increased by the use of a smaller maximum aggregate size. The explanation of this behavior lies in the fact that in the division of large aggregate particles into smaller particles, fracture takes place through the largest pores, which are thus eliminated. This division of large particles increases the strength of the concrete, but also increases its density.

Splitting tension tests usually show that the failure takes place through the coarse particles, this confirming good bond of the aggregate. Failure through the coarse aggregate particles was observed in all splitting tests conducted as a part of this study. High-strength lightweight aggregate concretes with some normal weight fine aggregate, with compressive strengths between 50 and $90 \mathrm{MPa}$, were found to have a flexural 
strength lower by up to $2 \mathrm{MPa}$ than normal weight concretes. The $72 \mathrm{MPa}$ lightweight aggregate concrete (LWC) in this study had a flexural strength $3.6 \mathrm{MPa}$ lower than the 75 MPa normal weight concrete (NWC). In the case of the splitting strength, the difference between the two concretes was about $1 \mathrm{MPa}$. The $72 \mathrm{MPa}$ LWC in this study had a splitting strength of $0.7 \mathrm{MPa}$ lower than the $75 \mathrm{MPa}$ NWC.

The strong bond between the aggregate and the surrounding hydrated cement paste is the result of several factors. First, the rough surface texture of many lightweight aggregates is conducive to a good mechanical interlocking between the two materials. In some cases, there is often penetration of cement paste into the open surface pores in the coarse aggregate particles. The porous surface is a result of the crushing process of the porous aggregate. Second, the moduli of elasticity of the lightweight aggregate particles and of the hardened cement paste are similar. Consequently, no differential stresses between the two materials are induced. Third, the water absorbed by the aggregate at the time of mixing becomes, with time, available for the hydration of any unhydrated cement particles. As most of this additional hydration occurs in the aggregate-cement paste interface zone, the bond between the aggregate and the matrix becomes stronger.

One effect of the strong bond between lightweight aggregate and the matrix is the absence of early development of bond microcracking. A consequence of this absence is that the stress-strain relation is linear up to a larger percent of the ultimate strength than normal weight concrete. This is especially so in lightweight aggregate concretes which also contain silica fume and have a 28-day compressive strength of about $90 \mathrm{MPa}$. The modulus of elasticity of lightweight aggregate concrete can be expressed as a function of the density of the concrete, as well as of its compressive strength. For strengths up to 41 
$\mathrm{MPa}, \mathrm{ACI}$ 318-95 expresses the modulus of elasticity of concrete, $\mathrm{E}_{\mathrm{c}}$, in $\mathrm{GPa}$ as $E_{c}=43 \times 10^{-6} \rho^{1.5} \sqrt{f^{\prime}{ }_{c}}$ where $f^{\prime}{ }_{c}$ is the standard cylinder strength in $\mathrm{MPa}$, and $\rho$ is the density of the concrete in $\mathrm{kg} / \mathrm{m}^{3}$. This expression applies to concretes having densities between 1440 and $2480 \mathrm{~kg} / \mathrm{m}^{3}$. Using the ACI 318-95 expression results in an elastic modulus of $19.4 \mathrm{GPa}$ for the $43.4 \mathrm{MPa}$ lightweight aggregate concrete $\left(f^{\prime}{ }_{c}=0.95 f_{\mathrm{c}}\right.$ where $f_{\mathrm{c}}$ is the compressive strength of a $100 \mathrm{~mm} \times 200 \mathrm{~mm}$ cylinder). The density of the concrete was $1700 \mathrm{~kg} / \mathrm{m}^{3}$. The measured elastic modulus was $21.4 \mathrm{GPa}$ which is $10 \%$ larger than the calculated value.

For lightweight aggregate concrete with compressive strengths in the range of 60 to $100 \mathrm{MPa}$, the modulus of elasticity can be estimated by a Norwegian standard expression reported by Zhang and Gjörv as $E_{c}=9.5 f_{c}^{0.3} \times\left(\frac{\rho}{2400}\right)^{1.5}$, where $E_{c}$ is the modulus of elasticity in $\mathrm{GPa}, f_{\mathrm{c}}$ is the compressive strength of 100 by $200 \mathrm{~mm}$ cylinders in $\mathrm{MPa}$, and $\rho$ is the density of the concrete in $\mathrm{kg} / \mathrm{m}^{3}$. Using the Norwegian expression results in a modulus of elasticity of $26.6 \mathrm{GPa}$ for the $69.6 \mathrm{MPa}$ lightweight aggregate concrete used in the high-strength prestressed beams in this study. The measured modulus was $26.2 \mathrm{GPa}(1.5 \%$ lower than the estimated value). It can be noted that the lower modulus of elasticity of lightweight aggregate concrete allows the development of a higher ultimate strain, compared with normal weight concrete of the same strength.

From the standpoint of workability and strength, it is a common practice to use normal weight sand as fine aggregate, and to limit the nominal size of the lightweight coarse aggregate to a maximum of $19 \mathrm{~mm}$. The lightweight aggregate concrete used in 
this study had normal weight sand as fine aggregate and a $9.5-\mathrm{mm}$ maximum lightweight coarse aggregate size.

\subsection{Durability of Lightweight Concrete}

Freeze/thaw durability of concrete, whether lightweight or normal weight, is achieved by protecting the paste (using air entrainment) and using durable aggregate. Expanded shale, clay, and slate aggregates are absorptive, however, they are very durable because they are composed of vitrified silicates. Laboratory results for structural LWC have indicated that high durability factors can be achieved after 300 cycles of freezing and thawing [ESCS1, U.S. Department of Transportation, 1988, Holm and Bremner, 1984, Holm, Bremner, and DeSouza, 1984]. Properly proportioned air entrained LWC is very durable. Lightweight aggregates have other unique properties that result in increased durability. Properties such as better elastic compatibility, ability to facilitate internal curing, improved bond between the LWA and the cement paste, and lower rigidity of the LWA, result in better durability characteristics. The stiffness of the LWA closely matches that of the air entrained cement paste. The elastic compatibility results in lower stress concentrations at the aggregate-paste interface, which greatly reduces the tendency for cracking at the microscopic level. The quality of the interface is a factor in the long-term durability of the concrete. Studies have shown that the contact zone in LWA concrete is far superior to that in normal weight concrete [U.S. Department of Transportation, 1988, Holm, Bremner, and Newman, 1984, Holm, Bremner, and DeSouza, 1984, Holm and Vaysburd, 1984, Zhang and Gjörv, 1990, Zhang and Gjörv, 1991, Swamy and Lambert, 1981, Zhang and Gjörv, 1990, 1992, Wasserman and Bentur, 
1996, Sarkar et al., 1992]. Expanded shale LWA is extremely well bonded to the mortar matrix and this bond far surpasses that of the normal weight aggregate bond. Cracking and local areas of high calcium content are often observed in the contact zone in concrete with NWA.

\subsection{Mix Design of Structural Lightweight Concrete}

Greater absorption and higher rates of absorption control the mix design procedures for LWC and are the principal factors necessitating modification of proportioning methods. Damp aggregates are preferable to dry aggregates because they absorb less water during mixing and reduce the possibility of slump loss [ACI 1987, 1991, Holm, 199?].

Damp aggregates have a lower tendency to segregate in storage. Absorbed water should be accounted for in mixture proportioning. When using LWA that have low initial moisture contents $(8-10 \%)$ and relatively high rates of absorption, it is desirable to mix the aggregates with $1 / 2$ to $2 / 3$ of the mixing water for a small period prior to the addition of the cement and air-entraining agent in order to minimize slump loss.

Concrete in which saturated LWA are used may be more vulnerable to freezing/thawing than concrete made with damp or dry LWA. This problem may be deterred if the concrete is allowed to lose its excess water after curing, prior to exposure, and is allowed to develop sufficient strength to withstand freezing and thawing conditions. In laboratory trial mixes using the specific gravity method, the specific gravity of the LWA should be determined at the moisture content expected during production. 
A well-graded aggregate will have a continuous distribution of particle sizes producing a minimum void content and will require a minimum amount of cement paste. This will result in an economical use of cement and provide the maximum available strength with minimum volume change due to drying and shrinkage.

The largest total volume of aggregate in a unit volume of concrete is achieved when [ACI 1991]:

a) The coarse aggregate is well graded from the largest to the smallest sizes.

b) When particles are rounded to cubical in shape.

c) When the surface texture is least porous.

Mixes with coarse aggregates that are angular in shape, more porous in texture, and may have some particle sizes missing, require a smaller volume of aggregates. The same factors also affect the percentage of fine aggregate required with a minimum percentage of fine aggregates being associated with a rounded or cubical shape and smooth texture.

In most cases, when well-graded, normal weight sand is used to replace lightweight fine aggregate, the proportions of coarse LWA may be increased. Strength may be increased by reducing the nominal maximum size of the aggregate without increasing the cement content.

The bulk specific gravity of the various size fractions of LWA usually increases as the particle size decreases. Some coarse aggregate may float on water, whereas material passing the No. 100 sieve $(0.15 \mathrm{~mm})$ may have a specific gravity approaching that of normal weight sand. It is the volume occupied by each fraction, and not the 
weight of the material retained on each sieve, that determines the void content and the cement paste content, and influences the workability of the concrete.

Two methods of mixture proportioning are described in ACI 211.2-91 [ACI 1991]. In Method I, the weight method, concrete is proportioned on the basis of an approximate water-cementitious materials ratio relationship when the absorption of the lightweight aggregate is known or determined. This method uses the fact that the sum of the weights per unit volume of all the materials in a mixture is equal to the total weight of the same mixture. If the weight of the particular concrete per unit volume, which contains the aggregate in question, is known or can be estimated from the specific gravity factor of the aggregate, the weight of the LWA in that volume of the concrete can be determined.

Method II (Volumetric Method) is used when the net water-cement ratio of LWC mixtures cannot be established with sufficient accuracy to be used as a basis for proportioning. This is due to the problems in finding the amount of total water that is absorbed in the aggregate and is thus not available for immediate reaction with the cement. In this case, LWC mixes are proportioned to achieve a desired consistency rather than on a water-cement ratio basis when the weight method is used. Mixture proportions are determined on a trial and error basis with a desired cement amount and slump. The merits of both methods of mixture proportioning are further discussed in Chapter 4.

Air-entrainment is recommended in most LWC as is the case with normal weight concrete. It enhances workability, improves resistance to freeze-thaw cycles and deicer chemicals, decreases bleeding, and tends to help overcome minor grading deficiencies. 
These effects can be achieved with a dosage of not lest than $4 \%$ of air. The recommended amount of air for LWC is $4-6 \%$ when a maximum size aggregate is $19 \mathrm{~mm}$ $(3 / 4 ")$ and $4.5-7.5 \%$ when the maximum size is $10 \mathrm{~mm}(3 / 8 ")$.

The strength of LWC may be reduced by higher air contents. At normal air contents (4-6\%) the reduction is small if slumps are $127 \mathrm{~mm}(5 \mathrm{in}$.$) , or less and cement$ contents are used as recommended [ACI 1991]. The volumetric method of measuring air (ASTM C173) is the most reliable method to determine the air content of fresh concrete containing LWA.

For a given aggregate the best method to select the amount of material for a trial mix is to use the proportions previously established for a similar mix. Such proportions can be obtained from the aggregate supplier. The initial mix can be adjusted to meet the specific job conditions. The job specification may dictate some or all of the following.

1. Minimum cement or cementitious materials content

$2 \quad$ Air content

3. Slump

4. Nominal maximum size aggregate

5. Unit Weight

6. Type of placement (pump, bucket, belt conveyor)

7. Strength

8. Other requirement such as strength over design, admixtures, and special types of cement and aggregates 


\subsection{Shear Strength of Concrete Beams}

A shear failure of a beam is rarely a failure due to shearing force alone. Rather, a shear failure is most commonly caused by a combination of shearing force and bending moment. Occasionally, axial load and/or torsion can be contributing factors in a shear failure. However, only shear failures due to combined bending and shear are within the scope of this study.

Each structural member exhibits different modes of cracking and failure, although the mechanisms by which shear is transferred within the member may be the same. One of the major variables affecting the mode of failure is $\frac{\mathrm{a}}{\mathrm{d}}$ or $\frac{\mathrm{M}}{\mathrm{V} \mathrm{d}}$, where a is the distance from concentrated load to the support; $d$ is the depth of the tensile reinforcement; $M$ is the moment at the section; and $\mathrm{V}$ is the shear force at the section. As $\frac{\mathrm{M}}{\mathrm{Vd}}$ changes, the relative magnitude of flexural stresses, shear stresses, and vertical stresses vary, and subsequently the behavior changes.

Reinforced concrete and prestressed concrete beams of moderate slenderness $\left(\frac{a}{d}\right.$ or $\frac{\mathrm{M}}{\mathrm{Vd}}=2$ to 6 ) develop inclined cracks due to a combination of shear and flexural stresses. The shear failures in deep beams differ considerably from those in normal beams. Much steeper inclined cracks develop in deep beams than in normal beams. This changes the relative importance of the various shear transfer mechanisms as compared to the normal beams.

The types of shear transfer mechanisms in reinforced concrete members include 

a) shear stress in the uncracked concrete $\left(\mathrm{V}_{\mathrm{cz}}\right)$
b) interface shear transfer (aggregate interlock) $\left(\mathrm{V}_{\mathrm{ay}}\right)$
c) dowel action $\left(V_{d}\right)$
d) arch action
e) shear reinforcement $\left(V_{s}\right)$

The total shear resistance of a reinforced concrete beam may be expressed as

$$
V_{t}=V_{s}+V_{c z}+V_{d}+V_{a y} \text {. }
$$

A prestressed concrete member with a change of vertical alignment of the tendon profile would have an additional component of shear resistance that would account for the upward force due to prestressing.

\subsection{Shear Tests}

Presently there is a limited number of tests on high strength lightweight aggregate concrete beams. Walraven and Al-Zubi conducted a series of shear tests on reinforced concrete I-shaped beams made of various types of lightweight aggregate (Walraven and Al-Zubi, 1995). The objective of their study was to determine the applicability of the variable strut inclination method (VSIM) to calculate the shear strength of lightweight aggregate concrete beams. The key assumption of the VSIM is the presence of a sufficient amount of redistribution capacity in the web of the beam. The researchers postulated that since the crack surfaces in lightweight aggregate concrete are smoother than those in gravel aggregate concrete and that since lightweight aggregate concrete is more brittle in compression, lightweight aggregate concrete beams may not have sufficient redistribution capacity. 
The type of cross section and test setup used by Walraven and Al-Zubi is shown in Figure 2.1. The a/d ratio was kept nearly constant at approximately 2 for all specimens. Although this a/d ratio seems small, the presence of significant arch action was not supported by the crack patterns. Primary variables in the test program included concrete compressive strength, type of coarse aggregate, and amount of shear reinforcement. Concrete compressive cylinder strength $\left(\mathrm{f}_{\mathrm{c}}\right.$ ) varied between $17.6 \mathrm{MPa}$ and $46.3 \mathrm{MPa}$. The types of coarse aggregate included gravel, Lytag, Liapor, and Aardelite. Lytag, Liapor, and Aardelite are types of lightweight aggregate. The aggregate in the lightweight concrete mixes consisted of $40 \%$ coarse aggregate particles and $60 \%$ natural river sand. In the current study, the aggregate in the lightweight aggregate mixes consisted of between $45\left(f_{c}=72 \mathrm{MPa}\right)$ and $52 \%\left(f_{c}=44 \mathrm{MPa}\right)$ of coarse lightweight aggregate and between 55 and $48 \%$ of natural sand. The level of transverse reinforcement $\left(\rho_{\mathrm{sw}} \mathrm{f}_{\mathrm{sy}}\right)$ varied between 2.5 MPa and 16.5 MPa. The highest amount of transverse reinforcement corresponds to approximately three times the amount allowed in ACI 318-95. Specimen details, concrete properties, and results of the beam tests are shown in Table 2.1. Walraven and Al-Zubi concluded that the redistribution capacity of the LWC beams tested is equivalent to that of gravel-aggregate concrete beams and that the VSIM can be applied to calculate the shear strength of LWC beams. In other words, the lightweight aggregate concrete beams tested behaved similarly to the normal-weight aggregate concrete beams.

Re-examination of the test results of Walraven and Al-Zubi, shown in Table 2.2, indicate that as the amount of shear reinforcement decreases, the ratio of the shear capacity of LWC beams to the shear capacity of NWC beams with equal amounts of 
shear reinforcement decreases. The shear capacities shown in Table 2.2 are normalized with respect to the square root of the concrete cylinder strength. Figure 2.2 shows a trend that indicates that the shear strength of LWC beams with low amounts of shear reinforcement is critical. The shear strength of LWC beams with $\Delta_{\text {sw }} f_{\text {sy }}$ less than $2.5 \mathrm{MPa}$ needs to be investigated.

Table 2.1 also indicates that the 3 types of lightweight aggregate concrete evaluated by Walraven and Al-Zubi had nearly the same ratio of compressive strength to splitting tensile strength as that of the gravel-aggregate concrete specimens. The average value of $\frac{\sqrt{f_{c}^{\prime}}}{f_{c t}}$ for the LWC (Lytag, Liapor, and Aardelite) was only $4 \%$ higher than the average value of $\frac{\sqrt{f_{c}^{\prime}}}{f_{c t}}$ for the NWC (gravel). This characteristic may have caused the LWC beams and the NWC beams to behave similarly but it is not representative of all LWA.

It should be noted that most beams tested by Walraven and Al-Zubi had a concrete compressive strength less of than 27.58 MPa. The behavior of HSLC beams needs to be investigated to evaluate the combined influence of lightweight aggregate and a high strength concrete matrix on the shear strength of members with low to medium amounts of transverse reinforcement. Both of these factors affect the roughness of the inclined crack surfaces and hence the aggregate interlock and mode of failure. 


\subsection{Methods of Calculating Shear Strength}

Methods of calculating the shear strength of reinforced and prestressed concrete beams will be discussed in this section. Special considerations for lightweight aggregate concrete beams will be noted. Code methods of estimating the shear strength include the First Edition of the American Association of State Highway and Transportation Officials LRFD Bridge Design Specifications (AASHTO LRFD), the $16^{\text {th }}$ Edition of the American Association of State Highway and Transportation Officials Bridge Design Specifications (AASHTO, 1995), and the American Concrete Institute Building Code Requirements for Structural Concrete and Commentary (ACI 318-95, 1995).

\subsubsection{AASHTO LRFD}

The design procedures based on the sectional model specified in AASHTO LRFD are limited to the design of flexural regions. Flexural regions are defined as regions of components where it is reasonable to assume that plane sections remain plane after loading. Where lightweight aggregate concrete is used, the term $0.85 \sqrt{f_{c}^{\prime}}$ shall be substituted for $\sqrt{\mathrm{f}_{c}^{\prime}}$.

The nominal shear resistance, $V_{n}$, shall be determined as the lessor of:

$$
\begin{aligned}
& V_{n}=V_{c}+V_{s}+V_{p} \text {, and } \\
& V_{n}=0.25 f^{\prime}{ }_{c} b_{v} d_{v}+V_{p},
\end{aligned}
$$

where, $\mathrm{fN}_{\mathrm{c}}$ is the concrete compressive strength in $\mathrm{MPa}, \mathrm{b}_{\mathrm{v}}$ is the effective web width in mm taken as the minimum web width within the depth $d_{v}$, and $d_{v}$ is the effective shear depth in $\mathrm{mm}$. 
The contributions to the shear resistance from the concrete $\left(V_{c}\right)$ and vertical stimups $\left(\mathrm{V}_{\mathrm{s}}\right)$ are shown below:

$$
\begin{gathered}
\mathrm{V}_{c}=0.083 \beta \sqrt{f_{c}^{\prime}} b_{v} d_{v} \text { and } \\
\mathrm{V}_{\mathrm{s}}=\frac{A_{v} f_{y} d_{v} \cot \theta}{s},
\end{gathered}
$$

where $A_{v}$ is the area of shear reinforcement within a distance $s, f_{y}$ is the yield strength of the shear reinforcement, and $\mathrm{s}$ is the spacing of the shear reinforcement. Beta is related to the ability of the diagonally cracked concrete to transmit tension. Theta is the assumed angle of inclination of the inclined crack. Values for $\beta$ and $\theta$ are determined according to either the simplified procedure (non-prestressed concrete sections) or the general procedure (non-prestressed and prestressed concrete sections). These procedures are discussed in the next two sections.

\subsubsection{Simplified Procedure}

For non-prestressed sections which contain a minimum amount of transverse reinforcement or which have an overall depth less than $400 \mathrm{~mm}$, the following values of beta and theta are to be used:

$$
\beta=2.0 \text { and } \theta=45 \text { degrees. }
$$

Using the above values reduces the shear equations to a form similar to that of $\mathrm{ACI} 318$ 95. Where transverse reinforcement is required, the minimum area of steel is defined as 


$$
A_{v}=0.083 \sqrt{f_{c}^{\prime}} \frac{b_{v} s}{f_{y}},
$$

where $A_{v}$ is the area of shear reinforcement in $\mathrm{mm}^{2}$ within a distance $s$; $\mathrm{fN}_{c}$ is the concrete compressive strength in MPa; $\mathrm{f}_{\mathrm{y}}$ is the yield strength of the shear reinforcement in $\mathrm{MPa}$; and $\mathrm{s}$ is the spacing of the shear reinforcement in $\mathrm{mm}$.

\subsubsection{General Procedure}

The general procedure for calculating the shear strength of prestressed and nonprestressed beams focuses on the determination of two parameters, $\beta$ and $\theta$. As mentioned earlier, beta is related to the ability of diagonally cracked concrete to transmit tension. Theta is the angle of inclination of diagonal compressive stresses (or the angle of inclination of the assumed inclined crack). In general, beta and theta are functions of the strain in the longitudinal tensile reinforcement $\left(\varepsilon_{\mathrm{x}}\right)$ and the shear stress in the concrete (v). In general, as $\varepsilon_{\mathrm{x}}$ and $v$ increase, the shear strength decreases.

For sections containing transverse reinforcement, the term $\beta$ depends on the longitudinal strain in the flexural tension reinforcement $\varepsilon_{\mathrm{x}}$, the average shear stress at the section $\nu$, and the concrete compressive strength $\mathrm{fN}_{\mathrm{c}}$. Values of $\beta$ are determined from AASHTO LRFD Table 5.8.3.4.2-1 reproduced in Appendix A as Table A.l.

The average shear stress is calculated as

$$
v=\frac{V_{u}-V_{p}}{b_{v} d_{v}}
$$


where, $V_{u}$ is the factored shear force at the section; $V_{p}$ is the component in the direction of the applied shear of the effective prestressing force; $b_{v}$ is the effective web width; and $d_{v}$ is the effective shear depth.

The longitudinal strain in the reinforcement on the flexural tension side of the member shall be determined as

$$
\varepsilon_{x}=\frac{\frac{M_{u}}{d_{v}}+0.5 V_{u} \cot \theta-A_{p s} f_{p o}}{E_{s} A_{s}+E_{p} A_{p s}},
$$

where $M_{u}$ is the factored moment at the section; $A_{p s}$ is the area of prestressing steel; $E_{p}$ is the modulus of elasticity of the prestressing steel; $A_{s}$ is the area of non-prestressed tensile reinforcement; and $E_{s}$ is the modulus of elasticity of non-prestressed tensile reinforcement.

If the value of $\varepsilon_{x}$ calculated from Equation (2.9) is positive (tension), then the value is unchanged. However, if the calculated value of $\varepsilon_{\mathrm{x}}$ is negative (compression), then the magnitude of $\varepsilon_{x}$ must be reduced by a factor $F$. . The factor $F$. takes into account the stiffness of the concrete in compression and is calculated as

$$
F_{\varepsilon}=\frac{E_{s} A_{s}+E_{p} A_{p s}}{E_{c} A_{c}+E_{s} A_{s}+E_{p} A_{p s}},
$$

where $E_{c}$ is modulus of elasticity of the concrete and $A_{g c}$ is the gross area of the composite section.

In order to calculate the steel contribution $\mathrm{V}_{\mathrm{s}}$, the value of $\theta$ must be determined from AASHTO LRFD Table 5.8.3.4.2-1 reproduced in Appendix A as Table A.1. The 
value of $\theta$ is dependent on the longitudinal strain in the flexural tension reinforcement, the average shear stress at the section, and the concrete compressive strength.

For section not containing transverse reinforcement, the terms $\beta$ and $\theta$ are dependent on the longitudinal strain in the flexural tension reinforcement and the crack spacing parameter $s_{x}$. The value of $s_{x}$ is determined from AASHTO LRFD Figure C5.8.3.4.2-1. For example, for a member without transverse reinforcement and with concentrated longitudinal reinforcement at the flexural tension side, the value of $s_{\mathrm{x}}$ would be approximately equal to the effective shear depth $d_{v}$.

The AASHTO LRFD general method is based on the more complex modified compression field theory which attempts to include most of the parameters that affect shear strength. Traditional parameters include concrete compressive strength, geometry of cross-section, amount and strength of shear reinforcement, and amount and level of prestress. The modified compression field theory includes variables such as maximum aggregate size, crack width, crack spacing, strain in the longitudinal tensile reinforcement, and shear stress in the concrete in addition to the traditional variables.

\subsubsection{ACI 318-95/ AASHTO}

This section contains the shear strength models from the ACI 318-95. The ACI 318-95 and AASHTO are assumed the same unless otherwise noted. The AASHTO specifications for the shear resistance of reinforced and prestressed concrete are in general agreement with the provisions of ACI 318-95. 


\subsubsection{Reinforced Concrete}

The nominal shear strength $\left(\mathrm{V}_{\mathrm{n}}\right)$ of reinforced concrete beams according to $\mathrm{ACI}$ 318-95 is composed of a steel contribution $\left(V_{s}\right)$ and concrete contribution $\left(V_{c}\right)$ :

$$
V_{n}=V_{c}+V_{s}
$$

The method is based on a modified truss analogy with an empirical concrete contribution and a steel contribution that is calculated assuming a 45 degree angle of inclination of diagonal compressive stresses $(\theta)$. For members subject to shear and flexure only, the following simplified expression for the concrete capacity (ACI Equation 11-3) is given:

$$
V_{c}=\left(\frac{\sqrt{f_{c}^{\prime}}}{6}\right) b_{w^{\prime}} d,
$$

where $\mathrm{fN}_{\mathrm{c}}$ is the concrete compressive strength in $\mathrm{MPa}$; $\mathrm{b}_{\mathrm{w}}$ is the web width in $\mathrm{mm}$; and $\mathrm{d}$ is the distance from the extreme compression fiber to the centroid of the tensile steel in $\mathrm{mm}$.

The shear strength $V_{c}$ may also be calculated by the following more detailed expression (ACI Equation 11-5):

$$
V_{c}=\left(\sqrt{f_{c}^{\prime}}+120 \rho_{w^{\prime}} \frac{V_{u} d}{M_{u}}\right) \frac{b_{w} d}{7} \leq 0.3 \sqrt{f_{c}^{\prime}} b_{w} d,
$$

where $\Delta_{\mathrm{w}}=\mathrm{A}_{\mathrm{s}} / \mathrm{b}_{\mathrm{w}} \mathrm{d} ; \mathrm{A}_{\mathrm{s}}$ is the area of non-prestressed tension reinforcement; $\mathrm{V}_{\mathrm{u}}$ is the factored shear force at the section; and $\mathrm{M}_{\mathrm{u}}$ is the factored moment at the section; and where $\frac{V_{u} d}{M_{u}} \leq 1.0$ when computing $\mathrm{V}_{\mathrm{c}}$ according to the previous equation. 
If lightweight aggregate concrete is used, certain modifications to the concrete contribution apply. When the average splitting tensile strength of lightweight aggregate concrete $\left(f_{c t}\right)$ is not specified, all values of $\sqrt{f_{c}^{\prime}}$ affecting $V_{c}$ and $M_{c r}$ shall be multiplied by 0.85 for "sand-lightweight" concrete. "Sand-lightweight" concrete refers to concrete made with lightweight coarse aggregate and natural sand as its fine aggregate.

The contribution to the shear strength by the vertical shear reinforcement is calculated as

$$
V_{s}=\frac{A_{v} f_{y} d}{s}
$$

where $A_{v}$ is the area of shear reinforcement within a distance $s ; f_{y}$ is the yield strength of the shear reinforcement; and $s$ is the spacing of the shear reinforcement. Spacing of shear reinforcement placed perpendicular to the axis of the member shall not exceed $d / 2$ nor $600 \mathrm{~mm}$. The minimum area of shear reinforcement shall be computed by

$$
A_{v}=\frac{1}{3} \frac{b_{w} s}{f_{y}} .
$$

\subsubsection{Prestressed Concrete}

The shear strength of prestressed concrete members includes, in addition to a concrete term and steel term, a term associated with the vertical forces produced from prestressing. The critical location for shear is located a distance of $h / 2$ from the face of the support. The term $\mathrm{h}$ is defined as the overall depth of the member. The nominal shear strength is defined as

$$
V_{n}=V_{c}+V_{s} \text {. }
$$


The concrete contribution to the shear strength is calculated as the lower of

$$
\begin{gathered}
V_{c i}=\frac{\sqrt{f_{c}^{\prime}}}{20} b_{w} d+V_{d}+\frac{V_{i} M_{c r}}{M_{\max }} \geq\left(\frac{1}{7}\right) \sqrt{f_{c}^{\prime}} b_{w} d \text { and } \\
V_{c w}=0.3\left(\sqrt{f_{c}^{\prime}}+f_{p c}\right) b_{w} d+V_{p},
\end{gathered}
$$

where $f_{c}$ is the concrete compressive strength in MPa; $b_{w}$ is the web width in mm; and $d$ is the distance from the extreme compression fiber to the centroid of the tensile steel in $\mathrm{mm} ; \mathrm{V}_{\mathrm{d}}$ is the shear force at the section due to unfactored dead load; $\mathrm{V}_{\mathrm{i}}$ is the factored shear force at the section due to externally applied loads occurring simultaneously with $\mathrm{M}_{\max } ; \mathrm{M}_{\max }$ is the maximum factored moment at the section due to externally applied loads; $M_{c r}$ is the moment causing flexural cracking at the section due to externally applied loads; $f_{p c}$ is the compressive stress in the concrete; and $V_{p}$ is the vertical component of effective prestress force at the section.

The term $\mathrm{V}_{\mathrm{c}}$ is multiplied by a factor 0.85 for sand-lightweight concrete.

The steel contribution to the shear strength is calculated as

$$
V_{s}=\frac{A_{v} f_{s} d}{s},
$$

if vertical stirrups are used. Spacing of shear reinforcement placed perpendicular to the axis of the member shall not exceed $0.75 \mathrm{~h}$ or $600 \mathrm{~mm}$. The minimum area of shear reinforcement shall be

$$
A_{v}=\frac{50 b_{n^{\prime}} s}{f_{y}} .
$$


Table 2.1 Requirements of ASTM C 330, C 331, and C 332 for Dry Loose Unit Weight of Lightweight Aggregates

\begin{tabular}{|l|c|}
\hline \multicolumn{1}{|c|}{ Aggregate Size and Group } & Maximum Unit Weight, $\mathrm{kgm}^{3}\left(\mathrm{lb} / \mathrm{ft}^{3}\right)$ \\
\hline ASTM C 330 and C 331 & \\
Fine aggregate & $1120(70)$ \\
Coarse aggregate & $880(55)$ \\
Combined fine and coarse aggregate & $1040(65)$ \\
ASTM C 332 & \\
Group 1 & \\
Perlite & $196(12)$ \\
Vermiculite & $160(10)$ \\
Group 2 & \\
Fine aggregate & $1120(70)$ \\
Coarse aggregate & $880(55)$ \\
Combined fine and coarse aggregate & $1040(965)$ \\
\hline
\end{tabular}


Table 2.2 Specimen Details and Results of Beams Tested by Walraven and Al-Zubi

\begin{tabular}{|c|c|c|c|c|c|c|}
\hline Specimen & $\begin{array}{c}\text { Shear } \\
\text { Reinf. } \\
\text { Ratio } \\
\%\end{array}$ & $\begin{array}{c}\text { Concrete } \\
\text { Cylinder } \\
\text { Strength } \\
\mathrm{f}_{\mathrm{c}} \\
\mathrm{MPa}\end{array}$ & $\begin{array}{c}\text { Splitting } \\
\text { Tensile } \\
\text { Strength } \\
\mathrm{f}_{\mathrm{ct}} \\
\mathrm{MPa}\end{array}$ & $V_{u} \exp$ & $\begin{array}{c}\mathrm{V}_{\mathrm{u} \text { calc }} \\
\mathrm{VSIM} \\
\mathrm{kN}\end{array}$ & $\frac{V_{\text {uexp }}}{V_{u \text { calc }}}$ \\
\hline Gd301 & 0.430 & 22.7 & 2.27 & 359.5 & 354 & 1.02 \\
\hline $\mathrm{Gd} 30 \mathrm{~m}$ & 0.887 & 17.6 & 1.82 & 420.0 & 368 & 1.14 \\
\hline Gd30h & 1.450 & 24.7 & 2.46 & 470.0 & 466 & 1.01 \\
\hline $\operatorname{Lg} 301$ & 0.430 & 19.1 & 1.68 & 324.0 & 325 & 1.00 \\
\hline $\mathrm{Lg} 30 \mathrm{~m}$ & 0.887 & 28.4 & 2.64 & 520.0 & 512 & 1.02 \\
\hline Lg30h & 1.450 & 25.2 & 2.43 & 481.5 & 475 & 1.01 \\
\hline Lr30I & 0.430 & 27.4 & 2.44 & 330.0 & 388 & 0.85 \\
\hline $\mathrm{Lr} 30 \mathrm{~m}$ & 0.887 & 25.0 & 2.34 & 461.0 & 475 & 0.97 \\
\hline $\mathrm{Lr} 30 \mathrm{~h}$ & 1.450 & 23.2 & 2.32 & 541.0 & 475 & 1.14 \\
\hline Ae30l & 0.430 & 22.8 & 2.06 & 321.5 & 355 & 0.90 \\
\hline $\mathrm{Ae} 30 \mathrm{~m}$ & 0.887 & 21.7 & 2.08 & 457.5 & 431 & 1.06 \\
\hline $\mathrm{Ae} 30 \mathrm{~h}$ & 1.450 & 20.2 & 1.95 & 482.0 & 397 & 1.21 \\
\hline Lg60] & 0.660 & 43.4 & 2.96 & 517.0 & 551 & 0.94 \\
\hline $\operatorname{Lg} 60 \mathrm{~m}$ & 1.250 & 46.3 & 3.17 & 751.0 & 680 & 1.10 \\
\hline Lg60h & 2.700 & 45.7 & 2.84 & 881.0 & 706 & 1.25 \\
\hline
\end{tabular}

For the specimens an identifying code was used, denoting the type of concrete ( $\mathrm{Gd}=$ gravel, $\mathrm{Lg}=\mathrm{Lytag}, \mathrm{Lr}=\mathrm{Liapor}, \mathrm{Ae}=$ Aardelite), the target concrete cube strength (30 or $60 \mathrm{MPa})$, and the shear reinforcement ratio $(\mathrm{l}=$ low, $\mathrm{m}=$ medium, $\mathrm{h}=\mathrm{high})$. 
Table 2.3 Re-examination of the Results of the Beams Tested by Walraven and Al-Zubi

\begin{tabular}{|c|c|c|c|}
\hline Specimen & $\frac{\left(\frac{V_{u \exp }}{\sqrt{f_{c}}}\right)}{\left(\frac{V_{u \exp }}{\sqrt{f_{c}}}\right)_{N W C}}$ & $\frac{\sqrt{f_{c}}}{f_{c t}}$ & Average $\frac{\sqrt{f_{c}}}{f_{c t}}$ \\
\hline Gd30l & 1.00 & 2.10 & \\
\hline $\mathrm{Gd} 30 \mathrm{~m}$ & 1.00 & 2.31 & 2.14 \\
\hline Gd30h & 1.00 & 2.02 & \\
\hline Lg30l & 0.98 & 2.60 & \\
\hline $\mathrm{Lg} 30 \mathrm{~m}$ & 0.97 & 2.02 & 2.23 \\
\hline Lg30h & 1.01 & 2.07 & \\
\hline Lr30l & 0.84 & 2.15 & \\
\hline $\mathrm{Lr} 30 \mathrm{~m}$ & 0.92 & 2.14 & 2.12 \\
\hline $\mathrm{Lr} 30 \mathrm{~h}$ & 1.19 & 2.08 & \\
\hline Ae30l & 0.89 & 2.32 & \\
\hline $\mathrm{Ae} 30 \mathrm{~m}$ & 0.98 & 2.24 & 2.29 \\
\hline $\mathrm{Ae} 30 \mathrm{~h}$ & 1.13 & 2.30 & \\
\hline Lg601 & 1.04 & 2.23 & \\
\hline $\operatorname{Lg} 60 \mathrm{~m}$ & 1.10 & 2.15 & 2.25 \\
\hline Lg60h & 1.38 & 2.38 & \\
\hline
\end{tabular}




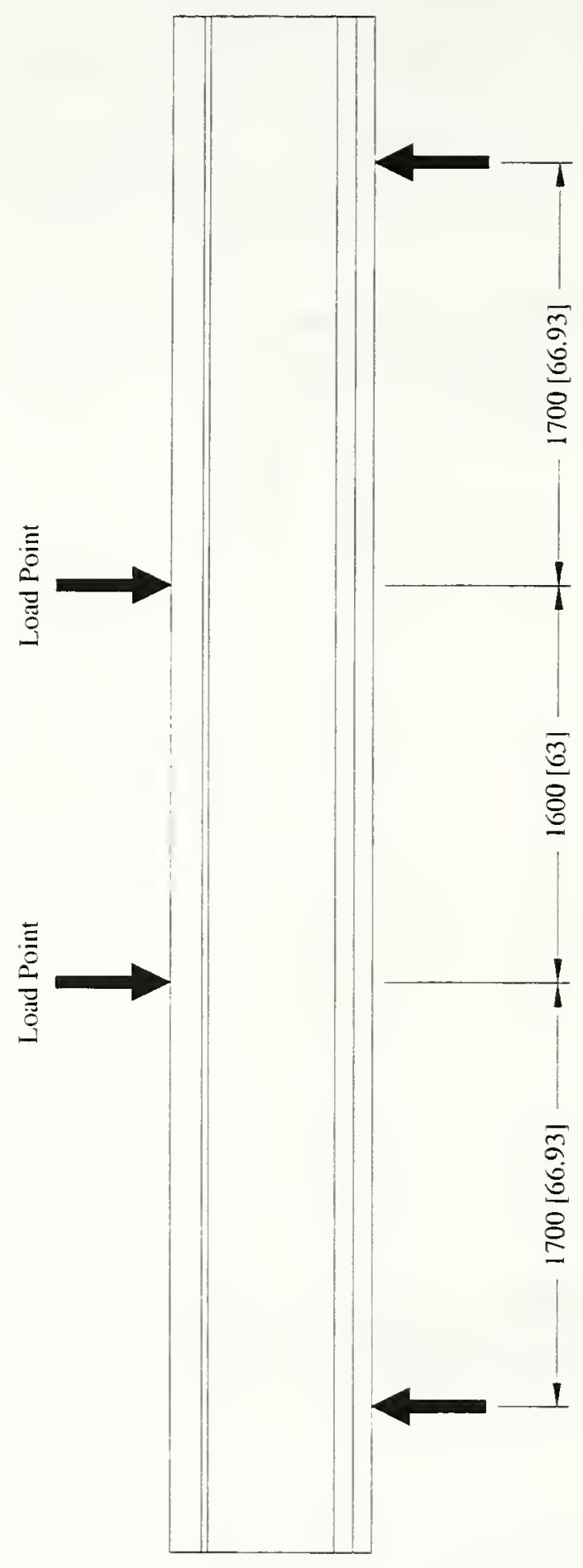

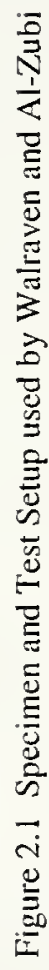

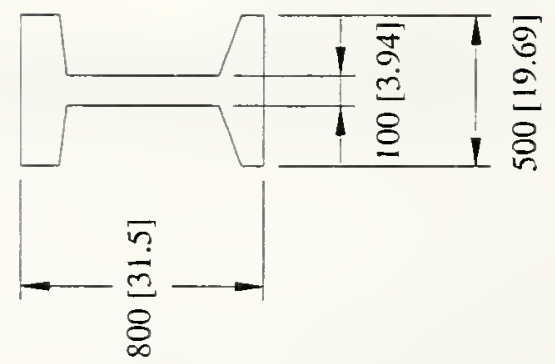




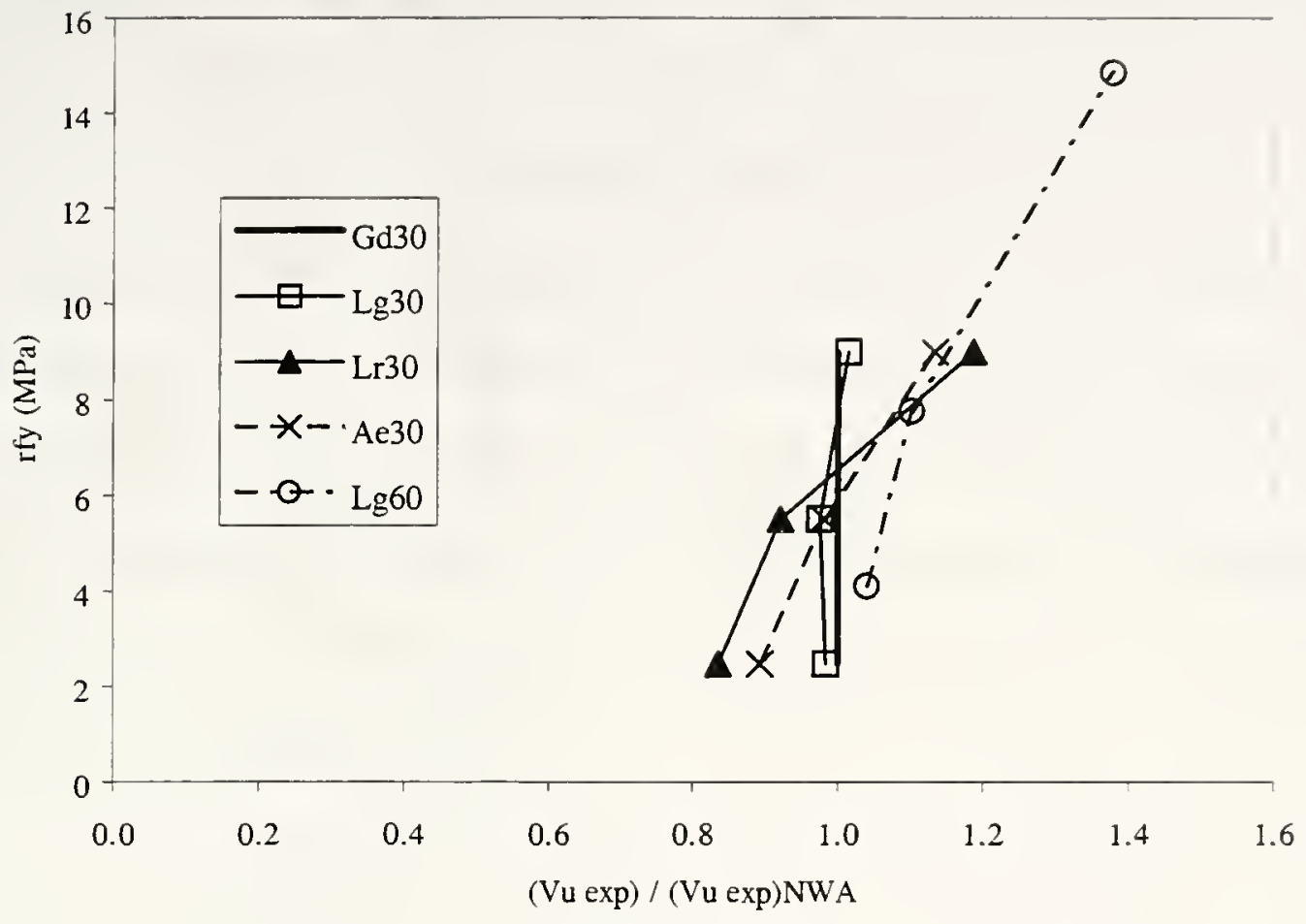

Figure 2.2 Comparison of Lightweight and Normal Weight Shear Strengths 


\section{CHAPTER 3. PROBLEM STATEMENT AND RESEARCH OBJECTIVES}

\subsection{Problem Statement}

Indiana Department of Transportation has successfully used a semi-lightweight concrete on several bridges to take advantage of the reduced dead load and decreased size of the members. However, to date, no systematic study has been performed to evaluate if the lightweight aggregate available from local sources meets criteria for bridge girders and deck applications, nor to develop a set of technical data that will allow for specifying these materials for bridges in Indiana .

As with any material, the use of lightweight concrete in bridge construction presents the challenge of balancing performance and cost. When compared to normal weight concrete the initial cost of lightweight concrete may be higher due to the higher cost of high-quality manufactured aggregate and a larger amount of cement required to achieve given strength. Higher cement content not only increases the initial cost but may also lead to other problems such as excessive shrinkage or thermal cracking. Frequently, stricter field quality control of moisture content, mixing, and placing is also required when lightweight aggregate is used. All of the above issues have to be addressed and satisfactorily resolved through proper material selection and mix optimization procedures before lightweight aggregate concrete becomes a viable and economical alternative for bridge applications.

Much research has been conducted on the shear strength of normal weight concrete girders. As a result of this research, specifications have been developed in which the shear strength is traditionally calculated as the sum of a concrete contribution 
and a steel contribution. These specifications have historically provided realistic and safe estimates of shear strength with few exceptions. Few tests have been conducted on lightweight aggregate high strength concrete prestressed beams, however, and the variations in materials used in these tests make it difficult to extrapolate those findings of to beams with different lightweight aggregates.

It is generally accepted that the shear strength of lightweight aggregate concrete beams is lower than the shear strength of otherwise similar beams made of normal-weight concrete. This belief is reflected in the ACI and AASHTO code equations by a reduction factor that is applied to the concrete term of the shear strength equations. The new AASHTO LRFD presents a different approach to the calculation of shear strength based on the modified compression field theory. The modified compression field theory was developed from a relatively small sample of normal weight concrete beam studies. Although the AASHTO LRFD includes provisions for calculating the shear strength of lightweight aggregate concrete beams, there is not sufficient experimental data to fully determine if the code effectively accounts for differences in the shear strength of lightweight and normal weight aggregate concretes.

The reduction of shear strength in lightweight concrete beams results from three behavioral differences that merit further investigation. First, for a given compressive strength, lightweight aggregate concrete has a lower tensile strength than normal weight concrete. Because shear is essentially the result of diagonal tension, reduced tensile capacity translates into reduced shear capacity.

The second phenomenon concerns the characteristic failure surface of lightweight concrete along a crack. As a result of the relatively weak course aggregate and strong 
transition zone, lightweight aggregate concrete tends to fail through the aggregate rather than through the paste, which is typically the case in moderate strength concrete with normal weight aggregate. The smoother crack surfaces result in a reduction in aggregate interlock and subsequently a reduction in shear capacity.

Thirdly, the mechanical properties of the lightweight aggregate vary greatly depending on its origins. Naturally occurring aggregate and manufactured aggregates consist of a number of different materials, each having different properties (i.e., shale, slate or clay).

A detailed investigation of the applicability of the AASHTO LRFD to lightweight aggregate concrete is needed to ensure that these specifications provide safe and reasonable estimates of shear strength. This investigation requires the fabrication, testing and analysis of a number of specimens that will help determine the behavior of lightweight aggregate concrete bridge girders in shear.

\section{$\underline{3.2 \text { Research Objectives }}$}

The main objective of this study was to evaluate the key aspects, both material and structural of the utilization of lightweight aggregate concrete in construction of decks and girders for the State of Indiana. The main issues addressed in the materials phase of the study included the following:

- Evaluation of suitability of Indiana sources of LWA for bridge construction

- Development of desirable fresh and hardened target concrete properties. 
- Development of optimized lightweight aggregate concrete mixes to meet the target compressive strength of $42 \mathrm{MPa}$ and $69 \mathrm{MPa}$ for use in bridge girders, and $35 \mathrm{MPa}$ concrete for use in bridge decks.

The purpose of the structural phase of this study was to evaluate the shear strength of reinforced and prestressed concrete beams made of sand-lightweight aggregate concrete. The beams were made of normal strength concrete and high-strength concrete. The normal-strength concrete beams had a target concrete compressive strength of 41.4 $\mathrm{MPa}$ and the high-strength concrete beams had a target concrete compressive strength of 69.0 MPa. The specific objectives included:

- Evaluation of the influence of lightweight aggregate concrete on the shear strength of reinforced and prestressed concrete beams.

- Development of experimental data on the shear strength of lightweight aggregate normal and high concrete strength beams.

- Evaluation of the code and specification shear design methods for lightweight aggregate concrete beams.

\subsection{Scope of the Research}

The selected lightweight aggregates from those described in Section 4.2.1 were used in developing concrete mixes for bridge decks and girders as deemed appropriate. The fresh and hardened concrete properties were determined and the durability performance of the proposed mixes was also evaluated. The properties of fresh concrete evaluated included slump, plastic unit weight, and air content. The mechanical properties 
of hardened concrete tested included compressive strength, tensile strength, flexural strength, static and dynamic modulus of elasticity, and Poisson's ratio. The durability related parameters tested included air void distribution, freeze-thaw resistance, chloride permeability, resistance to scaling, and drying shrinkage.

Silica fume, fly ash, and Type III Portland cement were used in the development of the $69 \mathrm{MPa}$ lightweight aggregate concrete mixes for use in bridge girders. The silica fume was used as an addition (10\% by wt. of cement). The fly ash was also used as an addition ( $15 \%$ by wt. of cement). A combination of $10 \%$ silica fume addition and $15 \%$ fly ash replacement was evaluated for the purpose of obtaining $69 \mathrm{MPa}$ compressive strength at 28 days. The criteria for development of the high strength lightweight concrete mix was to produce a mix that utilized all lightweight aggregate as coarse aggregate, natural sand as fine aggregate, and to obtain a 28 day compressive strength as close as possible to the target strength. However, an alternative mix design was developed using partial replacement of lightweight coarse aggregate with limestone coarse aggregate. The slump, dry unit weight, and air content requirements were $11-20 \mathrm{~cm}, 1522-1843 \mathrm{~kg} / \mathrm{m}^{3}$ and $5-6 \%$ respectively.

In the development of the $42 \mathrm{MPa}$ lightweight concrete for bridge girders, no mineral admixtures were used. The requirement for slump, unit weight and air content were the same as in the case for the $69 \mathrm{MPa}$ lightweight aggregate concrete. However, the water-cement ratio used was 0.42 instead of 0.33 as was the case in the $69 \mathrm{MPa}$ mix. The mixes all contained lightweight aggregate as coarse aggregate and natural sand as fine aggregate. 
A comparison of compressive strength development of lightweight aggregate concrete using Type III and Type I cement was conducted. Type III cement is typically used in the fabrication of prestressed beams, but Type I cement has also been used in the fabrication of prestress beams.

The mix design of lightweight aggregate concrete for the bridge decks had a target compressive strength of $31 \mathrm{MPa}$. The slump requirement will be $13-21 \mathrm{~cm}$ to address the issue of pumpability. The air content and dry unit weight requirement will be $5-6 \%$ and $1522-1843 \mathrm{~kg} / \mathrm{m}^{3}$ respectively. Type I cement was used in the development of lightweight aggregate concrete for bridge decks. Silica fume was used at a dosage rate of $7 \%$ replacement of cement. Fly ash was used at $15 \%$ level as a replacement for cement. The water-cementitious ratio used was 0.42 except in the case of fly ash concrete which was 0.40 . The coarse aggregate used was all lightweight and natural sand was used as the fine aggregate.

The structural testing program included both a reinforced concrete beams series and a prestressed concrete beam series. The reinforced concrete beam series consisted of twelve rectangular beams with transverse reinforcement. Five of twelve beams were made of sand-lightweight aggregate concrete. The remaining seven beams were made of normal weight concrete. Concrete compressive strengths at time of testing varied between $43 \mathrm{MPa}$ and $75 \mathrm{MPa}$. The effects of aggregate, concrete compressive strength, and reinforcement details on the shear strength were studied in the reinforced concrete series.

The prestressed concrete beam series consisted of four precast pretensioned AASHTO Type I girders with cast-in-place composite slabs. The variables in this series 
included concrete compressive strength and amount of transverse reinforcement. The girders were made of sand-lightweight aggregate concrete and the slabs were made of normal weight aggregate concrete. 


\subsection{Introduction}

In order to meet the objectives of the research project, an experimental study was conducted to evaluate both fresh and hardened concrete properties made from selected lightweight aggregate as well as the shear strength of reinforced and prestressed lightweight aggregate concrete beams with and without shear reinforcement. Materials used, specimen details, and testing procedures used in the experimental study are described in the following sections.

\subsection{Materials}

\subsubsection{Aggregates}

A total of four different types of aggregates were used in this research program, two lightweight aggregates and two normal weight aggregates. The lightweight aggregate used included an expanded shale with maximum aggregate size of $1 \mathrm{~cm}(3 / 8$ in.) and sintered fly ash lightweight aggregate with maximum aggregate size of $1.3 \mathrm{~cm}$ (1/2 in). The requirements of ASTM C 330, C 331, and C 332 for dry loose unit weight of lightweight aggregates are given in Table 4.1.

The normal weight aggregate used included an INDOT Class A river gravel and crushed limestone. All aggregates had a maximum grain size of $2 \mathrm{~cm}(3 / 4 \mathrm{in}$.$) . The$ Haydite lightweight aggregate was obtained from the Brooklyn, Indiana plant while the Minergy lightweight aggregate was obtained from the Wisconsin Electric Power Company plant in Milwaukee, Wisconsin. The INDOT Class A river gravel was 
obtained from Vulcan Materials in Lafayette Indiana. The crushed limestone was obtained from the Delphi Quarry in Indiana.

\subsubsection{Haydite Lightweight Aggregate}

The Haydite lightweight aggregate is an expanded shale and contains all the necessary constituents for bloating [Hydraulic Press Brick Company, Expanded Shale, Clay and Slate Institute]. The shale is mined in open pits and delivered to the crusher by truck. The shale is then crushed to the proper size and conveyed into the storage silos. The shale is then introduced into the rotary kiln at the cold end and the kiln is fired at the discharge end with natural gas.

The material is retained in the kiln for approximately 1 hour after which the minerals are subjected to decomposition at high temperatures and gases are evolved. At the same time, the shale is being softened by a flame temperature of about $1204^{\circ} \mathrm{C}$ $\left(2200^{\circ} \mathrm{F}\right)$. The vitreous material that results contains large numbers of tiny nonconnecting cells formed from the gas. The expanded shale is discharged from the kiln into a grate cooler. To minimize segregation and to eliminate dust, the expanded shale is moistened. The clinker is crushed and screened to achieve a properly balanced gradation for different end uses. The chemical analysis of Haydite aggregate is given in Table 4.2.

The 24-hour absorption of Brooklyn Haydite is approximately $10 \%$ by weight. Absorption will vary substantially with length of time due to the non-connecting of cellular structure of the aggregate and the small size of the cells. The procedure for determining absorption in the case of NWA is not adaptable to LWA because of the difficulty in determining the saturated surface dry condition. 
The Haydite Aggregate is produced to comply with the physical and chemical requirements of the applicable ASTM standards except that the medium coarse size (B) is often supplied with additional eight mesh materials to achieve desired block textures. The medium fine size (AX) is too coarse to comply with the gradation requirements. The available sizes are as follows:

A - Fine gradation which is used for cast-in-place concrete, precast concrete, concrete building units, and for shortcrete applications.

AX - Medium fine gradation used where a coarse sand sized material is desired.

B - Medium coarse gradation used for cast-in-place concrete, precast concrete and concrete blocks

C - Coarse gradation used for cast-in-place concrete, precast concrete, and geotechnical fill.

BX - Blend of fine and medium coarse Haydite used where bin availability limits the use of separate sizes. In most cases it is intended for block production, but it can be also used in cast-in-place concrete. However, it should be limited to low density low strength roof fill.

L - Coarse gradation used for ground cover.

The gradation and unit weight for Haydite aggregate is given in Table 4.3. The size distribution of Haydite aggregate determined in the laboratory is given in Table 4.4. Haydite structural concrete is one-third lighter than NWC. Haydite structural concrete with design strengths of $42 \mathrm{MPa}(6000 \mathrm{psi})$ is used for cast-in-place, precast and prestressed concrete. Special mixes of $69 \mathrm{MPa}(10,000 \mathrm{psi})$ are available. Haydite fill concrete is one-half the weight of NWC with six times the insulating capacity. Haydite 
masonry units are the lightest high quality units available. Haydite concrete units are widely used for engineered masonry and exposed masonry for both above and below grade construction.

\subsubsection{Minergy Lightweight Aggregate}

Minergy LWA is a sintered fly ash. The LWA is an environmentally-positive recycled product. The Minergy process is the combustion of ash and municipal and industrial sludge into lightweight aggregates and energy [Minergy (2)]. The Minergy process accommodates a broad range of raw materials. Combustion ashes with high or variable unbumed carbon levels, ashes containing ammonia or other residuals from air emission controls, and sludges with varying levels of metals and organic compounds.

The raw ash material (coal combustion fly ash, bed ash, bottom ash and incinerator ash) are blended and combined with proprietary binders, then pelletized or extruded to appropriate size for specific applications.

Municipal wastewater sludge, paper mill sludge and other industrial sludge are easily used. Organics are totally destroyed in the mineralization process, while metals are fused into the inert mineral matrix of Minergy lightweight aggregate. The Minergy process can be readily adjusted to accommodate high volume wastes with a broad range of moisture contents and diverse chemical compositions.

The blended raw materials are mineralized in a high temperature rotary kiln rendering them inert and environmentally harmless. The sludge and carbon in the ash supply the majority of the heat. Minergy lightweight aggregate is a strong, low density aggregate that is superior to natural aggregates for lightweight structural concrete and 
lightweight concrete masonry units and meets ASTM standards for these applications. The typical applications of Minergy lightweight aggregate are precast $(1.3 \mathrm{~cm}(1 / 2 \mathrm{in}.) \mathrm{x}$ No. 4), Concrete blocks ( $1 \mathrm{~cm}(3 / 8 \mathrm{in}.) \times \mathrm{pan})$, and landscaping and drainage $(2 \mathrm{~cm}(3 / 4$ in.). Minergy lightweight aggregate is also used in insulating concrete as a lightweight and fire resistant mineral fill, or as landscaping ground cover. The Minergy process yields a higher value product than conventional quarried aggregates because of its lightweight, high strength and unique properties.

The size distribution for Minergy LWA is given in Table 4.5. The physical and chemical properties of Minergy LWA are given in Table 4.6. The physical properties determined in the laboratory for the lightweight aggregates are given in Table 4.7. A graphical presentation of the size distribution is given in Figure 4.1.

\subsubsection{Cement}

The lightweight aggregate concrete for use in bridge girders was produced using Type III cement from Lone Star Industries (Greencastle plant). For comparative purposes one girder mix was prepared using Type I cement from Lehigh Portland cement plant in Mitchell, Indiana. Type I cement from Lone Star Industries from the Oglesby, Illinois plant was used in the production of bridge deck concrete. The physical and chemical properties of the cements are given in Tables 4.8-4.13.

\subsubsection{Silica Fume and Fly Ash}

The silica fume used in this study was a Grace Construction Products Force 10,000 D densified microsilica. The fly ash used was a Montrose 95-031 class C fly ash. 
No chemical analysis was obtained from the supplier of the microsilica. The chemical characteristics of the fly ash are given in Table 4.14.

\subsubsection{Admixtures}

The high range water reducers (HRWR) used in this project included Mighty 150 admixture (Boremco: non-toxic aqueous solution of an unreactive synthetic organic chemical, Type F), and Daracem 19 admixture (Grace: Nepthaline Sulfonate Formaldehyde). The water reducer used was WRDA 82 (Grace: Lignosulfonate and Amine). The retarder used was Daratard 17 (Grace: Lignosulfonate and compound Carbohydrates). The air-entraining agent used was Daravair 1400 (Grace: aqueous solution of neutralized resin acids and rosin acids).

\subsection{Mixture Proportioning}

\subsubsection{Pretreatment Procedures for Lightweight Aggregate}

Pre-wetting of lightweight aggregates before batching is a recommended procedure. The methods of pre-wetting include:

1. Vacuum saturation

2. Thermal saturation - sprinkling of aggregate with cold water as aggregates comes out of kiln.

3 Use of sprinklers on stockpile.

Because of the high absorption of lightweight aggregates, it is advisable to have the moisture content of the aggregate above the 24-hour absorption. Aggregates used in this condition would not absorb mixing water but would add a quantifiable amount of 
mixing water to the mixture. To determine the moisture content of the aggregates, the rapid moisture content test is used. Knowing the moisture content and the 24-hour absorption, the surface moisture can be determined. The procedure involves the use of a hot plate and alcohol. The alcohol is used to slightly coat the aggregates in other to accelerate the drying process. The aggregates are stirred while on the hot plate to further accelerate the drying and to avoid localized overheating. Corrections can then be made to the amount of batch water added to the mixture at the time of batching.

However, if the moisture content of the lightweight aggregates is not determined, there can be no accurate account of the mixing water. The preferred practice is to batch lightweight concrete by the volumetric method. The process does not involve the determination of moisture content and therefore, no adjustment is made to the mixing water to compensate for the surface water. The damp loose unit weight of the aggregate is determined and batching done to desired slump. Adjustment for moisture is done only to the fine aggregate. A summary of the batching procedures is detailed in Table 4.1. Typically, the average field and lab determined moisture content of the lightweight aggregate after pre-conditioning is $25 \%$ and $20 \%$ respectively.

Because of the varying specific gravities of lightweight aggregate and the rigid quality control that is necessary to produce good quality lightweight concrete, the supplier of the aggregate should always be consulted when using their material. The Haydite and Minergy aggregates are shipped in a damp condition to the customers. The average recorded moisture content of the Haydite and Minergy aggregates received was $9 \%$ and $11 \%$ respectively. When pre-conditioning the aggregates in the field, the 
suppliers suggest that the contractors shut down the sprinkler system 24 hours before the aggregates are used.

The procedure adopted in this research was to precondition the aggregate in the moist room until the time of batching. The aggregate was placed in a perforated plastic container that allowed for significant run off of water. This ensured that there was not an accumulation of water at the bottom of the container, and an accurate value of the moisture content could be determined. The as-received aggregate was initially sprinkled with a sufficient amount of water, then left in the moist room until batching. The moisture content was determined (ASTM C566) by using the procedure for rapid moisture content determination. Once the moisture content is known the mixes can be proportioned based on the water-cement ratio method rather than on the volumetric method.

\subsubsection{Development of Mix Proportions}

This section outlines the development of mixture proportions for the girder and deck concretes for use in bridges. The structural testing plan required that the actual 28 day compressive strength be as close as possible to the target strength. Type III portland cement from Lone Star Industries was used in the development of lightweight and normal weight concretes for use in bridge girders. For comparison purposes, a LWC and NWC mix using the $69 \mathrm{MPa}(10,000 \mathrm{psi})$ mixture proportions was done using Type I cement from Lehigh Portland Cement Company. Type I cement from Lone Star Industries was used in the development of bridge deck concrete. Natural sand was used in all the mixes. The proposed mixes for girder and deck concrete are presented in Table 4.16. 


\subsubsection{Mixture Proportioning of Concrete for Girders}

Two target compressive strengths (42 and $69 \mathrm{MPa} / 6000$ and 10,000 psi) were

selected for use in bridge girders. The development program for the girder concrete is given in Figure 4.2.

The girder mixes with target compressive strength of $42 \mathrm{MPa}(6000 \mathrm{psi})$ were developed using gravel aggregates, and Haydite and Minergy lightweight aggregates. The mixture proportions are given in Table 4.17. None of the concretes in this series had any mineral admixtures, but HRWR, WR, RET, and AEA were used. The water - cement ratio used was 0.33 for the NWC, and 0.42 for the LWC mixes.

The mixture proportions for the $69 \mathrm{MPa}(10,000 \mathrm{psi})$ concrete mixes are given in Tables 4.18-4.22. The girder mixes with target compressive strength of $69 \mathrm{MPa}(10,000$ psi) were developed using gravel and limestone normal weight aggregates, and Haydite and Minergy lightweight aggregates. The control mix contained no mineral admixtures. A combination of LWA and limestone was used in the development of the semilightweight concrete. Concrete mixes with target compressive strength of $69 \mathrm{MPa}$ (10,000 psi) were made using:

a) Haydite aggregate ( $\mathrm{LWCH})$.

b) Haydite aggregate and silica fume (LWCHS)

b) A combination of Haydite and limestone aggregates (SM-LWCHL)

c) A combination of Haydite and limestone aggregates, and silica fume (SM-LWCHLS)

d) Haydite aggregate and fly ash (LWCHF)

e) Haydite aggregate, silica fume and fly ash (LWCHSF)

f) Minergy aggregate and silica fume (LWCMIS) 
Limestone and gravel were used in the development of the normal weight high strength concrete. Silica fume was used in the concrete mixes at a dosage rate of $10 \%$ addition by weight of cement. Fly ash was used in the concrete mixes at a dosage rate of $15 \%$ addition by weight of cement.

The development of the LWC with target compressive strength of $69 \mathrm{MPa}$ $(10,000 \mathrm{psi})$ presented the most challenges and a series of combinations of cement, silica fume, fly ash, and silica fume-fly ash were used to obtain an optimized mix.

\subsubsection{Mixture Proportioning of Concrete for Decks}

The concrete mixes selected for bridge deck applications should offer good resistance to the ingress of chloride ions, freeze and thaw cycles, and scaling. The selected target compressive strength was $31 \mathrm{MPa}(4500 \mathrm{psi})$. Some of the limitations and requirements adopted for the purposes of bridge deck concrete included:

1. Maximum amount of cement $356 \mathrm{Kg} / \mathrm{m}^{3}(600 \mathrm{pcy})$

2. Water - cementitious ratio of 0.42

3. Silica fume of $7 \%$ addition by weight of cement

4. Fly ash of $15 \%$ addition by weight of cement

The development program and mixture proportions for the bridge deck concrete are given in Figure 4.2 and Table 4.8-4.9 respectively. The water-cementitious ratio used was 0.42 except in the case of the fly ash mix which had a water-cementitious ratio of 0.40 . Silica fume and fly ash were added at $7 \%$ and $15 \%$ replacement by weight of cement respectively. A class A river gravel and Haydite aggregates were used in the development program for bridge deck concrete. For comparative purposes a series of reference concrete mixes were produced using current Indiana Department of 
Transportation (INDOT) specifications. Because of time constraints, Minergy aggregate was only implemented in the reference concrete series.

Four mixes were developed for bridge deck concrete and were produced using the following combinations.

1. Haydite aggregate concrete as the control.

2. A class A river gravel NWC.

3. Haydite aggregate concrete with $7 \%$ replacement of silica fume by weight of cement.

4. Haydite aggregate concrete with $15 \%$ replacement of fly ash by weight of cement.

The reference concrete mixes for bridge decks had the same proportions of silica fume and fly ash. The water-cementitious ratio was 0.443 and the total amount of cement was $391 \mathrm{~kg} / \mathrm{m}^{3}(658 \mathrm{pcy})$.

\subsubsection{Practical Issues Related to Field Mixture Production}

The field production of the high strength LWC (69 MPa) provided numerous challenges in the lab and in the field. It was desired that a $69 \mathrm{MPa}(10,000 \mathrm{psi}) \mathrm{mix}$ be developed that utilized all lightweight aggregate as the coarse aggregate and natural sand as the fine aggregate. The field trial of the $69 \mathrm{MPa}(10,000 \mathrm{psi})$ normal weight concrete presented some challenges that were more easily handled. The $42 \mathrm{MPa}$ LWC and NWC mixes were easily produced at the plant.

The practical issues concerning the field production of LWC were:

1. Method of material proportioning

2. Difficulties in plant production 


\subsubsection{Procedure Used for Batching Lightweight Concrete in the Field}

The LWA stockpile was pre-conditioned by the use of a sprinkler system and the water was shut off 24 hours prior to mixing. The moisture content of the LWA and sand were determined using the rapid moisture content test. With the moisture contents of LWA and sand, and the absorption of the LWA known, the necessary adjustments to the batch water were made to account for the surface moisture.

The LWA was transferred to the bin and the aggregate dispensed into the pan mixer. One-third of the mixing water was then poured into the mixer, followed by the sand, cement and silica fume if used. The ingredients were then mixed for about 2 minutes after which another one-third of water was added along with the AEA. The RET was then added. The remaining water was added followed by the HRWR. The HRWR was dispensed in one-third increments. Slump measurements were made after each dispensation of HRWR. Air content and unit weight measurements were made immediately after mixing was completed. The volume of concrete made was $1.5 \mathrm{~m}^{3}$ (2 cy).

\subsubsection{Method of Material Proportioning in The Field}

One of the earliest issues addressed was evaluating the merits of proportioning by the weight method versus the volumetric method for LWC that is preferred. Water-tocement ratio becomes critical when developing high strength concrete in the $69 \mathrm{MPa}$ range and for this reason the weight method was used in this project because a knowledge of the water - cement ratio was necessary. In the volumetric method, the concrete is batched to desired slump. The water-cement ratio is unknown and furthermore, water is added to the batch to obtain the desired slump. It is a well-known fact that water-cement 
ratio influences the compressive strength of concrete. Water-cement ratios as low as 0.27 are currently used in the development of high strength NWC and the development of high strength LWC is no exception.

The question of whether the reported value of water-cement ratio was the true value can be raised since the moisture content of the LWA varies considerably due to high absorption capacities of the aggregate. The method used for determining the moisture content of the lightweight aggregates adopted in this research project was as accurate as can be experimentally achieved.

High cement contents and dosage rates of HRWR were used in the development of the high strength LWC. In general, LWC requires a much higher cement amount than does NWC. Cement contents ranging from $415-564 \mathrm{~kg} / \mathrm{m}^{3}$ (700 pcy-950 pcy) were used with the higher limit yielding the desired compressive strength. The manufacturer's recommended dosage rate was $5-10 \%$ per $45 \mathrm{~kg}$ (100 lbs) cement was initially used. However, the manufacturer's recommended dosage rates could not achieve the desired workability and hence a suitable dosage rate had to be adopted in the lab and field.

\subsubsection{Difficulty in Plant Production of Lightweight Concrete}

The $69 \mathrm{MPa}$ LWC was successfully produced in the lab and the plant trial conducted. There were some difficulties in producing the lab proven mix at the plant. In the first trial batch conducted, no measurement of slump, unit weight, and air content could be taken because the resulting mix was very stiff (approximate slump was about $1.3 \mathrm{~cm}(0.5 \mathrm{in}$.$) and difficult to compact.$

The difficulty experienced at the plant during the production the $69 \mathrm{MPa}(10,000$ psi) LWC initiated a new test series to investigate the cement-admixture compatibility. 
Cement and chemical admixtures from the precast plant were brought into the lab and trial mixes were produced. In addition to the Mighty 150 obtained from the precast plant two other HRWR admixtures (Daracem-19 and Rheobuild) were also used in the tests series. The HRWRs were used at rates of $5.5 \mathrm{~kg} / \mathrm{m}^{3}, 6 \mathrm{~kg} / \mathrm{m}^{3}, 7 \mathrm{~kg} / \mathrm{m}^{3}$. These mixes had the same mixture proportions as the one used to produce the $69 \mathrm{MPa}$ LWC and had the following combinations of cement and chemical admixtures:

1. Precast plant cement and precast plant admixtures.

2. Precast plant cement and lab admixtures.

3. Lab cement and precast plant admixtures.

4. Lab cement and lab admixtures.

The concrete produced during these test series was evaluated for slump, unit weight, air content, strength, and initial and final setting times. Some general trends observed during these tests are as follows:

1. The precast plant cement and admixture combination resulted in poor slump.

2. The lab admixtures produced good slump, but in combination with precast plant cement did not yield target compressive strength.

3. A combination of lab cement and precast plant admixtures resulted in good strength.

4. At a dosage rate of $6 \mathrm{~kg} / \mathrm{m}^{3}$, the use of lab materials did not produce desired compressive strength.

5. At higher dosage rates of $7 \mathrm{~kg} / \mathrm{m}^{3}$ the desired compressive strength was obtained using lab materials. 
6. Using cement and admixtures from the lab resulted in the target compressive strength being achieved. It could be concluded that the lab admixtures had a more superplasticizing and retarding effect.

7. The use of precast plant admixtures resulted in lower strength, but the strength was comparable to mixes using the lab stock of Mighty 150.

8. At lower dosages, the lab Mighty 150 exhibited more superplasticizing effect.

9. At higher dosage rates, Darecem-19 gave a higher slump.

10. At lower dosages, lab Mighty 150 and Daracem-19 showed longer setting times.

11. Mighty 150 from the precast plant exhibited the quickest time to final set.

12. Daracem-19 produced the best overall properties.

In the initial stages the $69 \mathrm{MPa}(10,000 \mathrm{psi}) \mathrm{mix}$ development process, the only mineral admixture used was silica fume. At later stages, the use of other admixtures were also tried. These trials included a combination of fly ash ( $15 \%$ replacement by weight of cement) and silica fume (10\% addition by weight of cement). The fly ash mixes did not produce the desired compressive strength (see Chapter Six).

The LWC and NWC mixes with target compressive strength of $42 \mathrm{MPa}$ were successfully produced at the precast plant. The inherent problem of quality control was not a significant problem in obtaining the target compressive strength for these mixes. This is due to the fact that the target strengths were on the low end and these can typically be easily achieved. The development of the LWC with target compressive strength of 31 MPa for use in bridge decks was not as rigorous a task as the high strength LWC mix development. 


\subsubsection{Summary}

There are numerous conclusions that can be drawn from this project about mixture proportion development and successful production of LWC in the field. The ready mix and precast concrete industry must have a much better understanding of the procedures necessary to produce high strength LWC. Quality control is much stricter and extremely important when using lightweight aggregates. Prior to casting of the reinforced and prestressed beams with LWC, trial batches were done to ensure that quality control was at a premium so that reproducibility of the target compressive strengths could be achieved.

The procedure for pre-conditioning the aggregates should be always followed by users. Water-cement ratio is critical in the development of high strength LWC and batching by weight method with the use of HRWR is recommended. Knowing the moisture content of the aggregate will facilitate adjustments to the batch water, thus maintaining the desired water-cement ratio.

It is the author's opinion that a better understanding by plant personnel of the procedures needed to be followed, and better communication with plant personnel is vital to the successful production of high strength LWC. The belief on the part of the plant personnel that the high strength LWC could be achieved using all LWA, all contributed to overall better quality control and enabled the achievement of the desired results.

\subsection{Concrete for Beam Specimens}

Details of the concrete used in the reinforced and prestressed specimens are discussed in the following sections. 


\subsubsection{Concrete for Reinforced Concrete Specimens}

Eleven of the twelve specimens were cast at the CSR Hydro Conduit precast plant in Lafayette, IN. The remaining specimen was cast in the Kettelhut Structural Engineering Laboratory at Purdue University. Type III and Type I cement were used in the mixes at the precast plant and laboratory, respectively. The coarse aggregate used in the normal-weight specimens included river gravel and crushed limestone. A maximum aggregate size of $19 \mathrm{~mm}$ was used for the normal-weight specimens. The coarse aggregate in the sand-lightweight specimens consisted of Haydite. Haydite is a manufactured lightweight aggregate made of expanded shale. A maximum aggregate size of $9.5 \mathrm{~mm}$ was used for the sand-lightweight specimens. All specimens used natural sand as the fine aggregate.

Target concrete compressive strengths varied from $41.4 \mathrm{MPa}$ to $68.9 \mathrm{MPa}$. Concrete mix proportions are shown in Table 4.24. For the high-strength specimens, either fly ash or silica fume were added to the mixes. Hardened concrete properties at the time of testing are shown in Table 4.25. Concrete compressive strengths were determined from at least five $102 \mathrm{~mm} \times 203 \mathrm{~mm}$ cylinders. The moduli of rupture were determined from two to three $152 \mathrm{~mm} \times 152 \mathrm{~mm} \times 508 \mathrm{~mm}$ beams loaded at the third points. The splitting cylinder strengths were determined from two to three $102 \mathrm{~mm} \times 203$ mm cylinders loaded on their sides. The elastic moduli were determined from at least two $102 \mathrm{~mm} \times 203 \mathrm{~mm}$ cylinders loaded to a stress of $40 \%$ of the compressive strength. Deformation of the middle region of the cylinder was measured with a compressometer. 


\subsubsection{Concrete for Prestressed Concrete Specimens}

All prestressed girder specimens were cast at the CSR Hydro Conduit precast plant in Lafayette, IN. Type III cement was used in all mixes at the precast plant. The coarse aggregate consisted of Haydite particles with a maximum aggregate size of 9.5 mm. All specimens used natural sand as the fine aggregate. For the high-strength specimens, silica fume was added to the mixes.

Target concrete compressive strengths varied from $41.4 \mathrm{MPa}$ to $68.9 \mathrm{MPa}$. Hardened concrete properties at the time of testing are shown in Table 4.26. Concrete compressive strengths were determined from at least five $102 \mathrm{~mm}$ x $203 \mathrm{~mm}$ cylinders. The moduli of rupture were determined from two to three $152 \mathrm{~mm} \times 152 \mathrm{~mm} \times 508 \mathrm{~mm}$ beams loaded at the third points. The splitting cylinder strengths were determined from two to three $102 \mathrm{~mm}$ x $203 \mathrm{~mm}$ cylinders loaded on their sides. The elastic moduli were determined from at least two $102 \mathrm{~mm} \times 203 \mathrm{~mm}$ cylinders loaded to a stress of $40 \%$ of the compressive strength. Deformation of the middle region of the cylinder was measured with a compressometer. Concrete mix proportions are shown in Table 4.27.

The concrete for the cast-in-place slab was a normal-weight concrete provided by a ready-mix concrete supplier. The concrete was a Class $\mathrm{C}$ mix according to INDOT specifications. Wabash River gravel with a maximum aggregate size of $19 \mathrm{~mm}$ was used in the mix. Hardened concrete proportions for the girders and slabs are shown in Table 4.26.

\subsection{Reinforcement}

Material properties, both measured and idealized, of the mild and high-strength reinforcement used in the reinforced concrete and prestressed concrete specimens are 
described below. Note that reinforcement sizes and grades are given by their English designations.

\subsubsection{Reinforcement for Reinforced Concrete Specimens}

The longitudinal reinforcement used in the reinforced concrete specimens consisted of Grade 60 No. 8 and No. 9 uncoated deformed steel bars. Typical stressstrain relationships for the longitudinal reinforcement are shown in Figures 4.4 and 4.5. Grade 40 No. 3 uncoated deformed steel bars were used for the transverse reinforcement in the test region. A typical stress-strain relationship for the No. 3 stirrups is shown in Figure 4.6. At least five samples of reinforcing bars were tested in tension for each type and size of reinforcement. During the tension tests, steel strains were measured with electrical resistance strain gages and an extensometer. Grade 60 No. 4 uncoated deformed steel bars were used as the transverse reinforcement outside the test region. A stress-strain relationship was not determined for the No. 4 bars.

\subsubsection{Reinforcement for Prestressed Concrete Specimens}

In the prestressed girders, the mild tensile longitudinal reinforcement consisted of Grade 60 No. 7 and No. 8 uncoated deformed steel bars. Typical stress-strain relationships for the longitudinal reinforcement are shown in Figures 4.7 and 4.8. Grade 60 No. 3 uncoated deformed steel bars were used for the transverse reinforcement. A typical stress-strain relationship for the No. 3 stirrups is shown in Figure 4.9. At least five samples of reinforcing bars were tested in tension for each type and size of reinforcement. During the tension tests, steel strains were measured with electrical resistance strain gages and an extensometer. Grade 60 No. 5 uncoated deformed steel 
bars were used as the longitudinal reinforcement in the upper flange of the girders. A stress-strain relationship was not determined for the No. 5 bars. The prestressing steel consisted of $1 / 2$ in. special low-relaxation 7 -wire prestressing strand. An idealized stressstrain relationship for the strand is shown in Figure 4.10.

The reinforcing steel used in the deck slab consisted of Grade 60 No. 5 uncoated deformed steel bars. A stress-strain relationship for the No. 5 bars was not determined.

\subsection{Description of Specimens}

Sizes, reinforcement details and construction of the sixteen specimens are described in the following sections.

\subsubsection{Reinforced Concrete Specimens}

The reinforced concrete beam series comprised 12 rectangular specimens. The variables in the reinforced concrete beam series were the type of coarse aggregate, concrete compressive strength, and the amounts and distributions of transverse and longitudinal reinforcement. The types of coarse aggregate that were used included river gravel, crushed limestone, and lightweight aggregate. Target concrete compressive strengths varied from 41.4 MPa to $68.9 \mathrm{MPa}$.

\subsubsection{Dimensions}

The nominal dimensions and reinforcement details of the test specimens are shown in Figures 4.11 to 4.14 . The reinforcing steel cages and formwork for the reinforced concrete beams were fabricated in the Kettelhut Structural Engineering Laboratory at Purdue University. The beams were $3353 \mathrm{~mm}$ in length. The test regions 
of the beams were $356 \mathrm{~mm}$ in height and $356 \mathrm{~mm}$ in width. Outside the test regions, the beams were $356 \mathrm{~mm}$ in height and $457 \mathrm{~mm}$ in width.

\subsubsection{Reinforcement Details}

The amounts of transverse reinforcement $\left(\mathrm{rf}_{\mathrm{yw}}\right.$ where $\mathrm{r}=\mathrm{A}_{\mathrm{v}} / \mathrm{bs}$ ) varied from 1.2 MPa to $1.8 \mathrm{MPa}$ corresponding to a stirrup spacing of $d / 2$ and $d / 3$, respectively. The longitudinal reinforcement ratios $\left(\rho=A_{s} / b d\right.$ for tension and $\rho^{\prime}=A_{s}{ }^{\prime} / b d$ for compression) were kept constant at approximately 0.0235 . The longitudinal bars were distributed over the upper and lower width of the beams in 4 specimens and bundled in the four corners in the remaining 8 specimens. Specimen details are summarized in Table 4.28.

The upper and lower rows of longitudinal reinforcement were continuous through the length of the specimens. The longitudinal reinforcement consisted of No. 8 and No. 9 uncoated deformed steel bars. Grade 40 No. 3 uncoated deformed steel bars were used for the transverse reinforcement in the test region. Grade 60 No. 4 uncoated deformed steel bars were used as the transverse reinforcement outside the test region. The transverse reinforcement in the test region consisted of double-leg and triple-leg closed stirrups with 135 degrees bends at the ends. The 4 different reinforcing details for the test regions are shown in Figure 4.15. Two sets of lifting hooks made of deformed bars were located $305 \mathrm{~mm}$ outside the test region to assist in handling the specimens. The reinforcing cages were tied together with plastic ties.

\subsubsection{Casting and Curing}

The formwork was made of wood and soaked with form release oil prior to casting. Care was taken not to spray the reinforcement with the form release oil. Eleven of the 
twelve specimens $(1-9,11,12)$ were cast at the precast plant, as shown in Figures 4.16 and 4.17. The remaining specimen (10) was cast at the laboratory, as shown in Figures 4.18 and 4.19. The concrete was placed in one lift and consolidated with hand held vibrators. Samples of the concrete were taken with $102 \mathrm{~mm} \times 204 \mathrm{~mm}$ cylinders, $152 \mathrm{~mm} \times 305$ mm cylinders, and $152 \mathrm{~mm} \times 152 \mathrm{~mm} \times 508 \mathrm{~mm}$ beams. The cylinders were plastic and had plastic covers. The flexure beam molds were steel. After casting, the concrete was covered with wet burlap and plastic for approximately $3-7$ days. The specimens cast at the precast plant were transported to the laboratory approximately 1 to 2 weeks after casting. Samples were kept in the same condition as the specimens.

\subsubsection{Prestressed Concrete Specimens}

The prestressed concrete beam series comprised 4 specimens. Each specimen was composed of an AASHTO Type I pretensioned girder and a cast-in-place topping slab designed to act compositely with the girder. The variables in the prestressed concrete beam series included concrete compressive strength and amount of web reinforcement. The girders consisted of sand-lightweight aggregate (Haydite) concrete and the slabs consisted of normal weight aggregate (river gravel) concrete. Target concrete compressive strengths of the pretensioned girders varied from $41.4 \mathrm{MPa}$ to $68.9 \mathrm{MPa}$.

The specimens were designed to fail in shear compression. Shear tension failures were avoided by placing the transfer length zone behind the support region. Flexural failures were avoided by supplementing the prestressing strand with sufficient mild deformed reinforcing bars and horizontal shear failures were avoided by placing a sufficient amount of shear connectors between the girder and slab. 


\subsubsection{Dimensions}

The nominal dimensions and reinforcement details of the test specimens are shown in Figures 4.20 to 4.24. Each specimen comprised an AASHTO Type I pretensioned girder and a cast-in-place deck slab.

\subsubsection{Reinforcement Details}

The amounts of web reinforcement $\left(\mathrm{rf}_{\mathrm{y}}\right)$ varied from 0 to $0.92 \mathrm{MPa}$. The larger amount of web reinforcement corresponds to a value close to the maximum stirrup spacing allowed according to the LRFD AASHTO 1994. Specimen details are summarized in Table 4.24 and reinforcement details are shown in Figures 4.25 and 4.26.

\subsubsection{Casting and Curing}

The AASHTO Type I girders were fabricated at the CSR Hydro Conduit Corporation precast plant in Lafayette, IN. All reinforcing steel was cut and bent by Hydro Conduit personnel. After the individual No. 3 stirrups were fabricated for Specimens PC6S and PC10S, electrical resistance strain gages were attached to the stirrups at the Kettelhut Structural Engineering Laboratory of Purdue University. After returning the instrumented stirrups to the precast plant, the reinforcing bar cages were tack welded together. The forms used to cast the girders were steel. The bottom prestressing strands were initially tensioned to $124 \mathrm{MPa}$. The reinforcing cages were placed into the forms and the top prestressing strands were fed through the mild reinforcement in the upper flange. All strands were then fully tensioned and seated with a stress of $1396 \mathrm{MPa}\left(0.75 \mathrm{f}_{\mathrm{pu}}\right)$. The additional mild longitudinal reinforcing bars were tied to the underside of the second lower layer of prestressing strands. Before closing the 
forms, form-release oil was sprayed on the side forms. Photographs of the reinforcing cages and prestressing strand layout prior to casting are shown in Figures 4.27 and 4.28.

Concrete was delivered to the prestressing beds by a chute from a concrete buggytype vehicle. Concrete was placed in two lifts and vibrated in place by hand-held vibrators. Specimens PC6N and PC6S were produced from two different batches with the same mix proportions. Specimens PC10N and PC10S were produced from the same batch of concrete. After the concrete compressive strength had reached at least 27.6 $\mathrm{MPa}$, the prestressing force was transferred to the concrete. The transfer of prestress was accomplished by slowly detensioning the jacking end of the strands and flame-cutting the other end of the strands with an acetylene torch as shown in Figure 4.29. The girders were then removed from the prestressing beds and moved to the precast plant storage yard. The girders were supported at their ends and were exposed to the weather for approximately one to two weeks until they were transported to the laboratory.

After the girders were moved to the laboratory, the falsework for the cast-in-place slab slabs was erected. The falsework consisted of steel overhang jacks that were suspended from each side of the girders and wooden formwork that rested on the overhang jacks, as shown in Figure 4.30. The cast of the slab utilized unshored construction. Before placing the reinforcing bar cage, form-release oil was sprayed onto the bottoms and sides of the wooden formwork. The tops flanges of the girders, which were roughly scored in the transverse direction at the precast plant, were cleaned before casting. The reinforcing bars for the slab consisted of Grade 60 No. 5 uncoated deformed reinforcing bars placed longitudinally and transversely at $127-\mathrm{mm}$ spacings. Twenty five millimeter slab bolsters supported the reinforcing bars and provided a $25 \mathrm{~mm}$ clear cover 
on the underside of the deck slab. In order to provide a continuous length of bar along the length of the entire beam, two $4572 \mathrm{~mm}$ No. 5 bars were lap spliced at midspan (splice length of $2515 \mathrm{~mm}$ ). The transversal and longitudinal bars were in the lower and upper layers of reinforcement, respectively. The reinforcing mat for the slab is shown in Figure 4.31.

The slab concrete was pumped into the slab formwork. Samples were taken from the mix to determine the concrete compressive strength at the time of testing. The concrete in the slab and samples was consolidated using a hand-held vibrator.

\subsection{Instrumentation}

The types of instrumentation used in the experimental program and the measurements taken are described in the following sections.

\subsubsection{Instrumentation of Reinforced Concrete Specimens}

Instrumentation consisted of electrical resistance strain gages, linear variable differential transformers (LVDT), load cells, and mechanical dial gages. Lucas Schaevitz DC-operated LVDTs were used to measure vertical deflections at locations shown in Figures 4.32 and 4.33. Model DC-E 500 LVDTs were used at the support locations and had a range of $\pm 13 \mathrm{~mm}$. Model DC-E 2000 LVDTs were used at locations 2,3,4 and 6 and had a range of $\pm 51 \mathrm{~mm}$. The electrical resistance strain gage system was provided by the Micro-Measurements Division of Measurements Group, Inc., located in Raleigh, N.C. Type CEA-06-250UN-350 foil gages were installed on the longitudinal reinforcing bars. Type CEA-06-125UN-350 foil gages were installed on the longitudinal and transverse reinforcing bars. The steel reinforcing bars were prepared for gage installation 
by grinding the ribs smooth, sanding the area, and cleaning it with M-Prep Conditioner A and M-Prep Neutralizer 5A. Type EA-06-20CBW-120 foil gages were attached to the concrete surface. Concrete gages had $51 \mathrm{~mm}$ gage lengths and 120-ohm resistances. MBond 200 adhesive and 200 Catalyst-C was used to attach the strain gages to the steel and concrete. Twenty-six gage 3-wire unshielded flat cable was connected to each strain gage. After the gages were attached to the surface and electrically connected to the cable, the gages were coated with polyurethane, wax, and a sealant (M-Coat J resin) to prevent moisture and dirt from damaging the gages. Strain gage locations for each specimen are shown in Figures 4.34 to 4.37.

A Megadac 200 - 200 Channel Data Acquisition System provided by Optim Electronics Corporation recorded all electronic measurements (electrical resistance strain gages, load cells, and LVDTs). The data acquisition system was controlled by a desktop computer running OPUS 200 (Optim Users Software). A photograph of the data acquisition unit and the LVDT power supply is shown in Figure 4.38.

Mechanical dial gages were also used to measure vertical deflection of the specimen and end slip of the longitudinal reinforcing bars. Typical dial gage layouts are shown in Figures 4.39 and 4.40. The mechanical dial gages used to measure vertical deflection were placed underneath the east side of the test specimen at the same longitudinal locations as the LVDTs. Gages that measured deflection at the support locations had 0.2 inch ranges and precisions of 0.0001 inch. Gages that measured deflections at other locations and end slip had 1 inch ranges and precisions of 0.001 inch.

Electronic and mechanical Whittemore strain gages were used to measure the deformations on the concrete surface. The electronic Whittemore strain gage was 
modeled after the original mechanical Whittemore strain gage. The electronic gage used a $6.35-\mathrm{mm}$ LVDT to measure deformations. The two electronic gages had gage lengths of 127 and $179 \mathrm{~mm}$. Figure 4.41 shows the two electronic Whittemore strain gages. The mechanical Whittemore strain gage had a gage length of $254 \mathrm{~mm}$. Figure 4.42 shows the mechanical Whittemore strain gage. Circular steel targets were attached to the surface of the concrete with epoxy. The points of the Whittemore strain gages were placed in the targets to measure changes in length of the concrete surface. The locations of the Whittemore targets and measurements for the reinforced concrete beams are shown in Figures 4.43 to 4.51 . Beams 1 through 10 had an orthogonal grid of targets. Beams 11 and 12 had a series of rosettes (location shown in Figure 4.5.2).

\subsubsection{Instrumentation of Prestressed Concrete Specimens}

Instrumentation consisted of electrical resistance strain gages, linear variable differential transformers (LVDT), load cells, and mechanical dial gages. Lucas Schaevitz DC-operated LVDTs were used to measure vertical deflections at locations shown in Figure 4.53. Model DC-E 500 LVDTs were used at the support locations and had a range of $\pm 13 \mathrm{~mm}$. Model DC-E 2000 LVDTs were used at locations $2,3,4$ and 5 and had a range of $\pm 5 \mathrm{I} \mathrm{mm}$. The electrical resistance strain gage system was provided by the Micro-Measurements Division of Measurements Group, Inc., located in Raleigh, N.C. Type CEA-06-125UN-350 foil gages were installed on the longitudinal and transverse reinforcing bars. The method of attaching the strain gages was the same as that described in Section 4.7.1. Strain gage locations for each specimen are shown in Figures 4.54 to 4.58 . 
A Megadac 200 - 200 Channel Data Acquisition System provided by Optim Electronics Corporation recorded all electronic measurements (electrical resistance strain gages, load cells, and LVDTs). The data acquisition system was controlled by a desktop computer running OPUS 200 (Optim Users Software).

Mechanical dial gages were also used to measure vertical deflection of the specimen and end slip of the protruding prestressing strands. Typical dial gage layouts are shown in Figure 4.59. The mechanical dial gages used to measure vertical deflection were placed underneath the east side of the test specimen at the same longitudinal locations as the LVDTs, as shown in Figure 4.53. Gages that measured deflection at the support locations had 0.2 inch ranges and precisions of 0.0001 inch. Gages that measured deflections at other locations and end slip had 1 inch ranges and precisions of 0.001 inch. Slip gages are shown in Figure 4.60.

A mechanical Whittemore strain gage was used to measure the deformations on the concrete surface. The gage had a gage length of $254 \mathrm{~mm}$ and is shown in Figure 4.42 . The Whittemore targets, described in section 4.4.1, were arranged in a rosette pattern on the webs of the beams as shown in Figures 4.61 and 4.62 . 
Table 4.1 Requirements of ASTM C 330, C 331, and C 332 for Dry Loose Unit Weight of Lightweight Aggregates

\begin{tabular}{|l|c|}
\hline \multicolumn{1}{|c|}{ Aggregate Size and Group } & Maximum Unit Weight, $\mathrm{kg} / \mathrm{m}^{3}\left(\mathrm{lb} / \mathrm{ft}^{3}\right)$ \\
\hline ASTM C 330 and C 331 & $1120(70)$ \\
$\quad$ Fine aggregate & $880(55)$ \\
Coarse aggregate & $1040(65)$ \\
Combined fine and coarse aggregate & \\
ASTM C 332 & \\
& \\
Group 1 & $196(12)$ \\
Perlite & $160(10)$ \\
Vermiculite & \\
Group 2 & $1120(70)$ \\
Fine aggregate & $880(55)$ \\
Coarse aggregate & $1040(965)$ \\
Combined fine and coarse aggregate & \\
\hline
\end{tabular}

Table 4.2 Chemical Analysis for Haydite Lightweight Aggregate

\begin{tabular}{|c|c|c|c|c|c|c|}
\hline Silica & $\begin{array}{c}\text { Ferric } \\
\text { Oxide } \\
\left(\mathrm{Fe}_{2} \mathrm{O}_{3}\right)\end{array}$ & Alumina & $\begin{array}{c}\text { Calcium } \\
\text { Oxide } \\
\left(\mathrm{Al} \mathrm{O}_{3}\right)\end{array}$ & $\begin{array}{c}\text { Magnesium } \\
\text { Oxide } \\
(\mathrm{MgO})\end{array}$ & $\begin{array}{c}\text { Sulphur } \\
\text { Trioxide } \\
\left(\mathrm{SO}_{3}\right)\end{array}$ & $\begin{array}{c}\text { Alkalies } \\
\left(\mathrm{Na}_{2} \mathrm{O}\right)\end{array}$ \\
\hline $57.6 \%$ & 9.6 & 19.4 & 3.4 & 2.6 & 0.6 & 5.6 \\
\hline
\end{tabular}

Table 4.3 Gradation and Unit Weight of Haydite Lightweight Aggregate

\begin{tabular}{|c|c|c|c|}
\hline & Size & Sieve Range & $\begin{array}{c}\text { Approx. Unit Weight Loose Damp. } \\
\mathrm{kg} / \mathrm{m}^{3}(\mathrm{pcf})\end{array}$ \\
\hline Fine & A & No. 4 x pan & $804(50)$ \\
\hline Medium & AX & No. $4 \times$ pan & $804(50)$ \\
\hline Medium Coarse & B & $1 \mathrm{~cm}(3 / 8$ in. $) \times$ No. 4 & $675(42)$ \\
\hline Coarse & C & $1.3 \mathrm{~cm}(1 / 2$ in. $) \times$ No. 4 & $643(40)$ \\
\hline Blend & BX & $1 \mathrm{~cm}(3 / 8$ in. $) \times$ pan & $804(50)$ \\
\hline Landscape & L & $3.8 \mathrm{~cm}(11 / 2$ in. $) \times 1 / 2$ in. & $595(37)$ \\
\hline
\end{tabular}


Table 4.4 Size Distribution of Haydite Aggregate Determined in the Laboratory

\begin{tabular}{|c|c|c|}
\hline Sieve No. & \% Retained & \% Passing \\
\hline \hline 1 cm (3/8 in.) & 0 & 100 \\
4 & 70.4 & 29.6 \\
8 & 26.4 & 3.2 \\
16 & 2.8 & 0.4 \\
30 & 0.04 & 0 \\
50 & 0 & 0 \\
\hline
\end{tabular}

Table 4.5 Size Distribution of Minergy Aggregate Determined in the Laboratory

\begin{tabular}{|c|c|c|}
\hline Sieve No. & \% Retained & \% Passing \\
\hline \hline $1.3 \mathrm{~cm}(1 / 2$ in. $)$ & 0 & 100 \\
$1 \mathrm{~cm}(3 / 8$ in. $)$ & 1.12 & 98.9 \\
4 & 98.4 & 0.48 \\
8 & 0.48 & 0 \\
\hline
\end{tabular}

Table 4.6 Chemical and Physical Properties of Minergy Lightweight Aggregate

\begin{tabular}{|l|l|}
\hline \multicolumn{1}{|c|}{ Chemical } & \\
\hline \multicolumn{1}{|c|}{ Property } & \\
Organic Impurities (AASHTO T-21, ASTM C 40) & No Injurious organic Compounds \\
Staining (ASTM C 641) & \\
Loss on Ignition (ASTM C114) & Light \\
\multicolumn{1}{|c|}{ Physical } & $<0.1 \%$ \\
Clay Lumps (ASTM C 142) & \\
Unit Weight & $0.1 \%$ \\
Dry Loose & \\
Dry Rodded & $809 \mathrm{~kg} / \mathrm{m}^{3}(49.8 \mathrm{pcf})$ \\
Soundness :Magnesium Sulfate (ASTM C 88) & $869 \mathrm{~kg} / \mathrm{m}^{3}(53.5 \mathrm{pcf})$ \\
& No. $4-\mathrm{No}^{\prime} .100-0.6 \%$ loss \\
Fineness Modulus & $3.8 \mathrm{~cm}\left(1.5^{\prime \prime}\right)-$ No. $5-0.4 \%$ loss \\
Absorption & 5.96 \\
Bulk specific gravity & $21.60 \%$ \\
\hline
\end{tabular}


Table 4.7 Physical Properties of Lightweight Aggregates Determined in the Laboratory

\begin{tabular}{|c|c|c|}
\hline Physical Property & Haydite Aggregate & Minergy Aggregate \\
\hline 24-hr absorption & $14 \%$ & $20 \%$ \\
Dry Loose Unit Weight & $659 \mathrm{~kg} / \mathrm{m}^{3}(41 \mathrm{pcf})$ & $804 \mathrm{~kg} / \mathrm{m}^{3}(50 \mathrm{pcf})$ \\
Dry Rodded Unit Weight & $755 \mathrm{~kg} / \mathrm{m}^{3}(47 \mathrm{pcf})$ & $884 \mathrm{~kg} / \mathrm{m}^{3}(55 \mathrm{pcf})$ \\
Bulk Specific Gravity & 1.33 & 1.44 \\
\hline
\end{tabular}

Table 4.8 Chemical Analysis for Lone Star Type III Cement

\begin{tabular}{|c|c|c|c|c|c|c|c|}
\hline \multicolumn{1}{|c|}{ Test } & $\mathrm{MgO}$ & $\mathrm{SO}_{3}$ & Loss & $\begin{array}{c}\text { Insol } \\
\text { Res. }\end{array}$ & $\mathrm{C}_{3} \mathrm{~S}$ & $\mathrm{C}_{3} \mathrm{~A}$ & $\begin{array}{c}\text { Total } \\
\text { Alkali }\end{array}$ \\
\hline $\begin{array}{l}\text { ASTM C } \\
150-97\end{array}$ & $\begin{array}{c}\text { Max. } \\
6 \%\end{array}$ & $\begin{array}{c}\mathrm{Max} . \\
4.5 \%\end{array}$ & $\begin{array}{c}\text { Max. } \\
3.0 \%\end{array}$ & $\begin{array}{c}\text { Max. } \\
0.8 \%\end{array}$ & - & $\begin{array}{c}\text { Max. } \\
15 \%\end{array}$ & - \\
\hline Mill Test & $1.38 \%$ & $3.04 \%$ & $1.60 \%$ & $0.30 \%$ & $61 \%$ & $10 \%$ & $0.44 \%$ \\
\hline
\end{tabular}

Table 4.9 Physical Analysis for Lone Star Type III Cement

\begin{tabular}{ccc}
\hline Physical Property & ASTM C $150-97$ & Mill Test \\
\hline 1 day & Min. & \\
& $1800 \mathrm{psi}$ & $4100 \mathrm{psi}$ \\
& $12.4 \mathrm{MPa}$ & $28.28 \mathrm{MPa}$ \\
3 day & Min. & \\
& $3500 \mathrm{psi}$ & \\
Blaine & $24.1 \mathrm{MPa}$ & $1192 \mathrm{~m}^{2} / \mathrm{kg}\left(5960 \mathrm{ft}^{2} / \mathrm{lb}\right)$ \\
Expansion & - & 0 \\
Air Entrainment & Max. $0.8 \%$ & $8 \%$ \\
Vicat-Initial & Max. $12 \%$ & $85 \mathrm{minutes}$ \\
Vicat-Final & Min. 45 minutes & $*$ \\
\hline
\end{tabular}


Table 4.10 Chemical Analysis for Lone Star Type I Cement

\begin{tabular}{|l|c|c|c|c|c|}
\hline \multicolumn{1}{|c|}{ Test } & $\mathrm{MgO}$ & $\mathrm{SO}_{3}$ & Loss & Insol. Res. & $\mathrm{C}_{3} \mathrm{~A}$ \\
\hline \hline ASTM C & Max. & Max. & Max. & Max. & $*$ \\
$150-97$ & $6 \%$ & $3.5 \%$ & $3.0 \%$ & $0.75 \%$ & \\
\hline Mill Test & $1.9 \%$ & $4.4 \%$ & $2.0 \%$ & $0.22 \%$ & $9.8 \%$ \\
\hline
\end{tabular}

Table 4.11 Physical Analysis for Lone Star Type I Cement

\begin{tabular}{|c|c|c|}
\hline Physical Property & ASTM C 150-97 & Mill Test \\
\hline \multirow[t]{3}{*}{3 day } & Min. & \\
\hline & 1740 psi & 3650 psi \\
\hline & $12 \mathrm{MPa}$ & $25.2 \mathrm{MPa}$ \\
\hline \multirow[t]{3}{*}{7 day } & Min. & \\
\hline & 2760 psi & \\
\hline & $19 \mathrm{MPa}$ & \\
\hline \multirow[t]{2}{*}{ Wagner } & Min. & \\
\hline & $160 \mathrm{~m}^{2} / \mathrm{kg}\left(800 \mathrm{ft}^{2} / \mathrm{lb}\right)$ & $182 \mathrm{~m}^{2} / \mathrm{kg}\left(910 \mathrm{ft}^{2} / \mathrm{lb}\right)$ \\
\hline \multirow[t]{2}{*}{ Blaine } & Min. & \\
\hline & $280 \mathrm{~m}^{2} / \mathrm{kg}\left(1400 \mathrm{ft}^{2} / \mathrm{lb}\right)$ & $337 \mathrm{~m}^{2} / \mathrm{kg}\left(1685 \mathrm{ft}^{2} / \mathrm{lb}\right)$ \\
\hline Expansion & Max. $0.8 \%$ & $0.03 \%$ \\
\hline Air Entrainment & Max. $12 \%$ & $8.4 \%$ \\
\hline Vicat-Initial & Min. 45 minutes & 98 minutes \\
\hline Vicat-Final & Max. 375 minutes & 168 minutes \\
\hline
\end{tabular}


Table 4.12 Chemical Analysis for Lehigh Type I Cement

\begin{tabular}{|c|c|}
\hline Chemical & Percentage (\%) \\
\hline $\mathrm{SiO}_{2}$ & 21.45 \\
\hline $\mathrm{Al}_{2} \mathrm{O}_{3}$ & 4.68 \\
\hline $\mathrm{Fe}_{2} \mathrm{O}_{3}$ & 3.36 \\
\hline $\mathrm{CaO}$ & 65.16 \\
\hline $\mathrm{MgO}$ & 1.56 \\
\hline $\mathrm{SO}_{3}$ & 2.70 \\
\hline Ignition Loss & 0.83 \\
\hline Insoluble Residue & 0.11 \\
\hline Potential Compounds & 58 \\
$\mathrm{C}_{3} \mathrm{~S}$ & 18 \\
$\mathrm{C}_{2} \mathrm{~S}$ & 6.7 \\
$\mathrm{C}_{3} \mathrm{~A}$ & 10.2 \\
$\mathrm{C}_{4} \mathrm{AF}$ & 0.53 \\
\hline $\mathrm{Alkalies}$ & \\
$\mathrm{Na}_{2} \mathrm{O}$ equivalent & \\
\hline
\end{tabular}

Table 4.13 Physical Analysis for Lehigh Type I Cement

\begin{tabular}{lc}
\hline Physical Property & \\
\hline Blaine $\mathrm{m}^{2} / \mathrm{kg}$ & 361 \\
Autoclave Expansion (\%) & -0.04 \\
Time of Set Gilmore & 185 \\
Initial, minutes & 325 \\
Final, minutes & 6 \\
Air content (\%) & \\
1 day & $17.6 \mathrm{Mpa}(2550 \mathrm{psi})$ \\
3 day & $27.6 \mathrm{Mpa}(4000 \mathrm{psi})$ \\
7 day & $33.8 \mathrm{Mpa}(4890 \mathrm{psi})$ \\
28 day & $42.7 \mathrm{Mpa}(6190 \mathrm{psi})$ \\
\hline
\end{tabular}


Table 4.14 Chemical Analysis for Montrose Class C Fly Ash, Identification \# 95-031

\begin{tabular}{|c|c|}
\hline Chemical Compound & Percentage (\%) \\
\hline $\mathrm{SiO}_{2}$ & 40.44 \\
\hline $\mathrm{Al}_{2} \mathrm{O}_{3}$ & 18.19 \\
\hline $\mathrm{Fe}_{2} \mathrm{O}_{3}$ & 5.46 \\
\hline $\mathrm{CaO}$ & 22.76 \\
\hline $\mathrm{MgO}$ & 5.08 \\
\hline $\mathrm{LSO}_{3}$ & 1.33 \\
\hline $\mathrm{K}_{2} \mathrm{O}$ & 0.46 \\
\hline $\mathrm{Na}_{2} \mathrm{O}$ & 2.22 \\
\hline $\mathrm{TiO}_{2}$ & 1.32 \\
\hline $\mathrm{P}_{2} \mathrm{O}_{5}$ & 1.32 \\
\hline $\mathrm{Mn}_{2} \mathrm{O}_{3}$ & 0.05 \\
\hline $\mathrm{SrO}$ & 0.41 \\
\hline $\mathrm{LOI} @ 1050$ & 0.67 \\
\hline $\mathrm{Total}$ & 99.72 \\
\hline $\mathrm{LOI} @ 750$ & 0.48 \\
\hline $\mathrm{Na}_{2} \mathrm{O}$ (sol.) & 0.11 \\
\hline $\mathrm{K}_{2} \mathrm{O}$ (sol.) & 0.01 \\
\hline
\end{tabular}

Table 4.15 Batching Procedures

\begin{tabular}{|l|l|}
\hline \multicolumn{1}{|c|}{ Preferred Method } & \multicolumn{1}{c|}{ Purdue University } \\
\hline 1. Mixture proportioning done by volume & 1. Mixture proportioning done by weight, \\
with no handle on water-cement ratio & water-cement ratio determined \\
2. 24-hr pre-soaking of aggregate & 2. 24-hr pre-soaking of aggregate \\
3. Damp loose unit weight determined & 3. Damp loose unit weight is not needed \\
$\begin{array}{l}\text { 4. Batching done by adding water to } \\
\text { obtain desired slump }\end{array}$ & $\begin{array}{l}\text { 4. Rapid Moisture content test done } \\
\text { 5. Adjustments for moisture done only to } \\
\text { sand and not LWA }\end{array}$ \\
& f. Moisture adjustment made to LWA and \\
& 6. Determination of water-cement ratio to a \\
& reasonable degree of accuracy \\
\hline
\end{tabular}

Table 4.16 Proposed Mixes for Use in Bridge Girders and Decks

\begin{tabular}{|c|c|c|c|}
\hline $\begin{array}{c}\text { Type of } \\
\text { Concrete }\end{array}$ & $\begin{array}{c}\text { Normal Strength, Target } \\
42 \mathrm{MPa}(6000 \mathrm{psi})\end{array}$ & $\begin{array}{c}\text { High Strength, Target } \\
69 \mathrm{MPa},(10,000 \mathrm{psi})\end{array}$ & $\begin{array}{c}\text { Bridge Deck, Target 31 } \\
\mathrm{MPa}(4500 \mathrm{psi})\end{array}$ \\
\hline NWC & NWC & $\begin{array}{c}\text { NWC } \\
\text { (gravel and limestone } \\
\text { aggregate) }\end{array}$ & NWC \\
\hline LWC & H, MI & HS & H, HS, HF \\
\hline
\end{tabular}


Table 4.17 Mixture Proportions for $42 \mathrm{MPa}$ (6000 psi) Concrete Using Type III Cement for Use in Bridge Girders

\begin{tabular}{|c|c|c|c|}
\hline Materials $\left(\mathrm{kg} / \mathrm{m}^{3}\right)$ & NWC & LWCH & LWCMI \\
\hline Cement, & 303 & 421 & 421 \\
Water & 101 & 178 & 178 \\
Gravel & 1024 & 0 & 0 \\
Haydite & 0 & 653 & 0 \\
Minergy & 0 & 0 & 653 \\
Sand & 890 & 611 & 733 \\
HRWR & 4.7 & 1.68 & 0 \\
WR. & 0 & 0 & 0.56 \\
RET. & 0.55 & 0.55 & 0.55 \\
AEA & 0.39 & 0.28 & 0.28 \\
\hline W/C & 0.33 & 0.42 & 0.42 \\
\hline
\end{tabular}

Table 4.18 Mixture Proportions for NWC and SM-LWC, 69 MPa(10,000 psi) Using Type III Cement, Girder Mixes.

\begin{tabular}{|c|c|c|c|c|c|}
\hline $\begin{array}{c}\text { Materials } \\
\left(\mathrm{kg} / \mathrm{m}^{3}\right)\end{array}$ & NWC & NWC & NWC-Plant & $\begin{array}{c}\text { SM- } \\
\text { LWCHL }\end{array}$ & $\begin{array}{c}\text { SM- } \\
\text { LWCHLS }\end{array}$ \\
\hline Cement & 385 & 385 & 385 & 504 & 446 \\
Water & 128 & 128 & 128 & 136 & 148 \\
Silica Fume & 0 & 0 & 0 & 0 & 45 \\
Limestone & 0 & 982 & 0 & 412 & 412 \\
Gravel & 982 & 0 & 982 & 0 & 0 \\
Haydite & 0 & 0 & 0 & 242 & 242 \\
Minergy & 0 & 0 & 0 & 0 & 0 \\
Sand & 867 & 867 & 880 & 875 & 831 \\
HRWR & 4.7 & 4.7 & 4.7 & 6.4 & 5.46 \\
WR & 0 & 0 & 0 & 0.59 & 0 \\
RET & 0.55 & 0.59 & 0.55 & 0.59 & 0 \\
\hline W/C & 0.33 & 0.33 & 0.33 & 0.27 & 0.33 \\
\hline
\end{tabular}


Table 4.19 Mixture Proportions for High Strength LWC, 69 MPa (10,000 psi) Using Type III Cement, Girder Mixes.

\begin{tabular}{|c|c|c|c|}
\hline Materials $\left(\mathrm{kg} / \mathrm{m}^{3}\right)$ & LWCHS & LWCHS - Plant & LWCMIS \\
\hline Cement & 564 & 564 & 564 \\
Water & 186 & 186 & 169 \\
Silica Fume & 56 & 56 & 56 \\
Haydite & 526 & 526 & 0 \\
Minergy & 0 & 0 & 526 \\
Sand & 653 & 653 & 710 \\
HRWR & 6.44 & 8.4 & 8.2 \\
WR & 0 & 0 & 0.59 \\
RET & 0.59 & 0.59 & 0.59 \\
AEA & 0.21 & 0.21 & 0.20 \\
\hline W/C & 0.33 & 0.33 & 0.30 \\
\hline
\end{tabular}

Table 4.20 Mixture Proportions for LWC with Silica Fume and Fly Ash, $69 \mathrm{MPa}$ (10,000 psi), Type III Cement, Girder Mixes.

\begin{tabular}{|c|c|c|c|c|}
\hline $\begin{array}{c}\text { Materials } \\
\left(\mathrm{kg} / \mathrm{m}^{3}\right)\end{array}$ & LWCHS & LWCHF & LWCH & LWCHF \\
\hline Cement & 479 & 564 & 564 & 593 \\
Water & 186 & 186 & 186 & 186 \\
Silica Fume & 56 & 0 & 0 & 0 \\
Fly Ash & 85 & 85 & 0 & 89 \\
Haydite & 526 & 526 & 526 & 526 \\
Sand & 653 & 653 & 653 & 653 \\
HRWR & 7 & 4.2 & 6.0 & 7 \\
WR & 0.59 & 0.59 & 0.59 & 0.59 \\
RET & 0.59 & 0.59 & 0.59 & 0.59 \\
AEA & 0.28 & 0.28 & 0.28 & 0.28 \\
\hline W/C & 0.33 & 0.33 & 0.33 & 0.31 \\
W/CEM & 0.30 & 0.28 & 0.33 & 0.27 \\
\hline
\end{tabular}


Table 4.21 Mixture Proportions for LWC and NWC, $69 \mathrm{MPa}$ (10,000 psi), Type I Cement, Girder Mixes.

\begin{tabular}{|c|c|c|}
\hline $\begin{array}{c}\text { Material } \\
\left(\mathrm{kg} / \mathrm{m}^{3}\right)\end{array}$ & LWCHS & NWC \\
\hline Cement & 564 & 385 \\
Water & 186 & 128 \\
Silica Fume & 56 & 0 \\
Haydite & 526 & 0 \\
Limestone & 0 & 982 \\
Sand & 653 & 870 \\
HRWR & 6.44 & 3.64 \\
WR & 0 & 0 \\
RET & 0.59 & 0.59 \\
AEA & 0.21 & 0.28 \\
\hline W/C & 0.33 & 0.33 \\
\hline
\end{tabular}

Table 4.22 Mixture Proportions for LWC and NWC, Bridge Deck, Type I Cement

\begin{tabular}{|c|c|c|c|c|}
\hline $\begin{array}{c}\text { Material } \\
\left(\mathrm{kg} / \mathrm{m}^{3}\right)\end{array}$ & LWCH & LWCHS & LWCHF & NWC \\
\hline Cement & 356 & 331 & 303 & 303 \\
Water & 150 & 150 & 142 & 127 \\
Mineral & 0 & 25 & 53 & 0 \\
Admixture & 581 & 560 & 560 & 0 \\
Haydite & 0 & 0 & 0 & 0 \\
Minergy & 0 & 0 & 0 & 1023 \\
Gravel & 788 & 848 & 848 & 890 \\
Sand & 1.4 & 2.8 & 0.90 & 0.90 \\
HRWR & 0 & 0 & 0 & 0 \\
WR & 0 & 0 & 0 & 0 \\
RET & 0.28 & 0.28 & 0.28 & 0.28 \\
AEA & 0.42 & 0.42 & 0.40 & 0.42 \\
\hline W/CEM & & & & 0 \\
\hline
\end{tabular}


Table 4.23 Mixture Proportions for Reference LWC and NWC Bridge Deck, Type I Cement

\begin{tabular}{|c|c|c|c|c|c|}
\hline $\begin{array}{c}\text { Materials } \\
\left(\mathrm{kg} / \mathrm{m}^{3}\right)\end{array}$ & LWCH & LWCHS & LWCHF & LWCMI & NWC \\
\hline Cement & 391 & 364 & 292 & 391 & 391 \\
Water & 173 & 173 & 173 & 173 & 173 \\
Mineral & 0 & 27 & 99 & 0 & 0 \\
Admixture & 481 & 481 & 481 & 0 & 0 \\
Haydite & 0 & 0 & 0 & 640 & 0 \\
Minergy & 0 & 0 & 0 & 1023 \\
Gravel & 0 & 792 & 777 & 920 & 890 \\
Sand & 808 & 0 & 0 & 0 & 0 \\
HRWR & 0 & 0 & 0 & 0 & 0 \\
WR & 0 & 0 & 0 & 0 & 0 \\
RET & 0 & 0.28 & 0.28 & 0.28 & 0.28 \\
AEA & 0.28 & 0.443 & 0.443 & 0.443 & 0.443 \\
\hline W/CEM & 0.443 & 0 & &
\end{tabular}


Table 4.24 Concrete Mix Proportions of Reinforced Concrete Series

\begin{tabular}{|c|c|c|c|c|c|c|}
\hline \multirow[b]{2}{*}{ Component } & \multicolumn{6}{|c|}{ Concrete Mix Design (Amount per cubic meter) } \\
\hline & $\begin{array}{l}\text { 1-NWLA } \\
\text { 2-NWLB } \\
\text { 3-NWLC } \\
\text { 4-NWLD }\end{array}$ & $\begin{array}{l}\text { 5-LWLA } \\
\text { 6-LWLB } \\
\text { 7-LWLC } \\
\text { 8-LWLD }\end{array}$ & 9-NWLD & 10-NWHD & 11-LWHD & 12-NWHD \\
\hline Cement $(\mathrm{kg})$ & 303 & 422 & 386 & 447 & 565 & 440 \\
\hline Water $(\mathrm{kg})$ & 101 & 178 & 128 & 171 & 185 & 158 \\
\hline $\begin{array}{l}\text { Silica Fume } \\
(\mathrm{kg})\end{array}$ & - & - & - & - & 56 & 23 \\
\hline Fly Ash (kg) & - & - & - & 89 & - & - \\
\hline Limestone $(\mathrm{kg})$ & - & - & 984 & 1011 & - & 951 \\
\hline Gravel (kg) & 1026 & - & - & - & - & - \\
\hline Haydite $(\mathrm{kg})$ & - & 654 & - & - & 527 & - \\
\hline Sand $(\mathrm{kg})$ & 892 & 612 & 841 & 654 & 654 & 756 \\
\hline HRWR (ml) & 5002 & 1776 & 5002 & 4779 & 8880 & 4730 \\
\hline WR (ml) & - & 577 & & - & - & 192 \\
\hline RET (ml) & 577 & 577 & 577 & - & 622 & - \\
\hline $\mathrm{AEA}(\mathrm{ml})$ & 414 & 296 & 296 & - & 222 & 364 \\
\hline W/C & 0.33 & 0.40 & 0.33 & 0.32 & 0.33 & 0.34 \\
\hline Slump (mm) & 152 & 127 & 152 & 127 & 203 & 165 \\
\hline$\%$ Air & 6 & 5 & 5 & 4 & 5.5 & 6.6 \\
\hline $\begin{array}{l}\text { Unit Weight } \\
\left(\mathrm{kg} / \mathrm{m}^{3}\right)\end{array}$ & 2328 & 1702 & 2312 & 2456 & 2038 & 2350 \\
\hline
\end{tabular}


Table 4.25 Hardened Concrete Properties of Reinforced Concrete Series

\begin{tabular}{|l|c|c|c|c|}
\hline \multicolumn{1}{|c|}{ Specimen } & $\begin{array}{c}\text { Compressive } \\
\text { Strength }\end{array}$ & $\begin{array}{c}\text { Modulus of } \\
\text { Rupture }\left(\mathrm{f}_{\mathrm{r}}\right)\end{array}$ & $\begin{array}{c}\text { Splitting } \\
\text { Tensile } \\
\text { Strength }\left(\mathrm{f}_{\mathrm{ct}}\right)\end{array}$ & $\begin{array}{c}\text { Static Modulus } \\
\text { of Elasticity } \\
\left(\mathrm{E}_{\mathrm{c}}\right)\end{array}$ \\
\hline \hline 1-NWLA & 46.2 & 6.2 & 4.3 & 40000 \\
2-NWLB & 46.2 & 6.2 & 4.3 & 40000 \\
3-NWLC & 46.6 & 6.2 & 4.3 & 40000 \\
4-NWLD & 45.8 & 6.0 & 4.3 & 40000 \\
5-LWLA & 43.4 & 5.7 & 3.7 & 21440 \\
6-LWLB & 43.0 & 4.8 & 3.8 & 21440 \\
7-LWLC & 43.4 & 5.7 & 3.2 & 21440 \\
8-LWLD & 44.3 & 5.7 & 3.8 & 21440 \\
9-NWLD & 40.0 & 5.3 & 3.6 & - \\
10-NWHD & 60.1 & 6.2 & - & - \\
11-LWHD & 72.3 & 2.3 & 3.9 & 41510 \\
12-NWHD & 75.2 & 5.9 & 4.6 & \\
\hline
\end{tabular}


Table 4.26 Concrete Mix Proportions of Prestressed Concrete Series

\begin{tabular}{|c|c|c|}
\hline \multirow[b]{2}{*}{ Component } & \multicolumn{2}{|c|}{ Concrete Mix Design (Amount per cubic yard) } \\
\hline & $\begin{array}{l}\text { PC6N } \\
\text { PC6S }\end{array}$ & $\begin{array}{l}\text { PC10N } \\
\text { PC10S }\end{array}$ \\
\hline Cement (lbs) & 710 & 950 \\
\hline Water (lbs) & 300 & 311 \\
\hline Silica Fume (lbs) & - & 95 \\
\hline Haydite (lbs) & 1100 & 887 \\
\hline Sand (lbs) & 1030 & 1100 \\
\hline HRWR (oz) & 60 & 300 \\
\hline WR (oz) & 19.5 & - \\
\hline $\operatorname{RET}(\mathrm{Oz})$ & 19.5 & 21.0 \\
\hline AEA (oz) & 10.0 & 7.5 \\
\hline $\mathrm{W} / \mathrm{C}$ & 0.40 & 0.33 \\
\hline Slump (in) & 5 & 8 \\
\hline$\%$ Air & 5.0 & 5.5 \\
\hline Unit Weight (pcf) & 106 & 127 \\
\hline
\end{tabular}

Table 4.27 Hardened Concrete Properties of Prestressed Concrete Series

\begin{tabular}{|c|c|c|c|c|c|}
\hline & \multicolumn{4}{|c|}{ Girder } & Slab \\
\hline Specimen & $\begin{array}{c}\text { Compressive } \\
\text { Strength } \\
\\
\mathrm{MPa}\end{array}$ & $\begin{array}{c}\text { Modulus of } \\
\text { Rupture }\left(f_{r}\right) \\
\text { MPa }\end{array}$ & $\begin{array}{c}\text { Splitting } \\
\text { Tensile } \\
\text { Strength }\left(f_{\mathrm{ct}}\right) \\
\mathrm{MPa}\end{array}$ & $\begin{array}{c}\text { Static } \\
\text { Modulus of } \\
\text { Elasticity } \\
\left(\mathrm{E}_{\mathrm{c}}\right) \\
\mathrm{MPa}\end{array}$ & $\begin{array}{c}\text { Compressive } \\
\text { Strength } \\
\mathrm{MPa}\end{array}$ \\
\hline PC6N & 48.5 & 3.2 & 3.2 & - & 43.4 \\
\hline PC6S & 44.8 & 2.0 & 3.3 & - & 37.7 \\
\hline PC10N & 69.6 & 4.1 & 3.9 & 26200 & 41.7 \\
\hline PCIOS & 69.6 & 4.0 & 3.7 & 26200 & 34.2 \\
\hline
\end{tabular}


Table 4.28 Specimen Details of Reinforced Concrete Beam Series

\begin{tabular}{|l|c|c|c|c|c|}
\hline Specimen & $\begin{array}{c}\text { Target Concrete } \\
\text { Compressive } \\
\text { Strength } \\
\mathrm{MPa}\end{array}$ & $\mathrm{a} / \mathrm{d}$ & $\rho\left(\rho^{\prime}\right)$ & $\mathrm{r}$ & $\mathrm{r} \mathrm{f}_{\mathrm{yw}}{ }^{\mathrm{b}}$ \\
\hline \hline 1-NWLA & 41.4 & 2.14 & 0.0233 & 0.00393 & $\mathrm{MPa}$ \\
2-NWLB & 41.4 & 2.14 & 0.0233 & 0.00393 & 1.8 \\
3-NWLC & 41.4 & 2.16 & 0.0237 & 0.00393 & 1.8 \\
4-NWLD & 41.4 & 2.16 & 0.0237 & 0.00262 & 1.2 \\
5-LWLA & 41.4 & 2.14 & 0.0233 & 0.00393 & 1.8 \\
6-LWLB & 41.4 & 2.14 & 0.0233 & 0.00393 & 1.8 \\
7-LWLC & 41.4 & 2.16 & 0.0237 & 0.00393 & 1.8 \\
8-LWLD & 41.4 & 2.16 & 0.0237 & 0.00262 & 1.2 \\
9-NWLD & 41.4 & 2.16 & 0.0237 & 0.00262 & 1.2 \\
10-NWHD & 68.9 & 2.16 & 0.0237 & 0.00262 & 1.2 \\
11-LWHD & 68.9 & 2.16 & 0.0237 & 0.00262 & 1.2 \\
12-LWHD & 68.9 & 2.16 & 0.0237 & 0.00262 & 1.2 \\
\hline
\end{tabular}

a Specimen Identification Scheme: NWLA

Normal Weight Aggregate

Low Strength: Target Concrete Compressive Strength $=41.4 \mathrm{MPa}$

${ }^{\mathrm{b}} \mathrm{f}_{\mathrm{yw}}=460 \mathrm{MPa}$ 
Table 4.29 Specimen Details of Prestressed Concrete Beam Series

\begin{tabular}{|c|c|c|c|c|c|}
\hline Specimen & $\begin{array}{c}\text { Target Concrete } \\
\text { Compressive } \\
\text { Strength } \\
\mathrm{MPa}\end{array}$ & $\mathrm{a} / \mathrm{d}$ & $\begin{array}{c}\text { Prestressing } \\
\text { Details } \\
\text { Strands }\end{array}$ & $\mathrm{r}$ & $\begin{array}{l}\mathrm{If}_{\mathrm{yw}}{ }^{\mathrm{b}} \\
\mathrm{MPa}\end{array}$ \\
\hline $\mathrm{PC} 6 \mathrm{~N}^{\mathrm{a}}$ & 41.4 & 3.03 & $\begin{array}{c}2 \text { (Top) } \\
8 \text { (Bottom) }\end{array}$ & 0 & 0 \\
\hline PC6S & 41.4 & 3.03 & $\begin{array}{c}2 \text { (Top) } \\
8 \text { (Bottom) }\end{array}$ & 0.00183 & 0.92 \\
\hline PCION & 68.9 & 3.04 & $\begin{array}{c}2 \text { (Top) } \\
8 \text { (Bottom) }\end{array}$ & 0 & 0 \\
\hline PC10S & 68.9 & 3.04 & $\begin{array}{c}2 \text { (Top) } \\
8 \text { (Bottom) }\end{array}$ & 0.00183 & 0.92 \\
\hline
\end{tabular}

${ }^{a}$ Specimen Identification Scheme: PC6N

Prestressed Concrete Beam

$\underline{6} 000$ psi Target Concrete Compressive Strength

${ }^{b} \mathrm{f}_{\mathrm{yw}}=\frac{\text { No Stirrups }}{502 \mathrm{MPa}}$

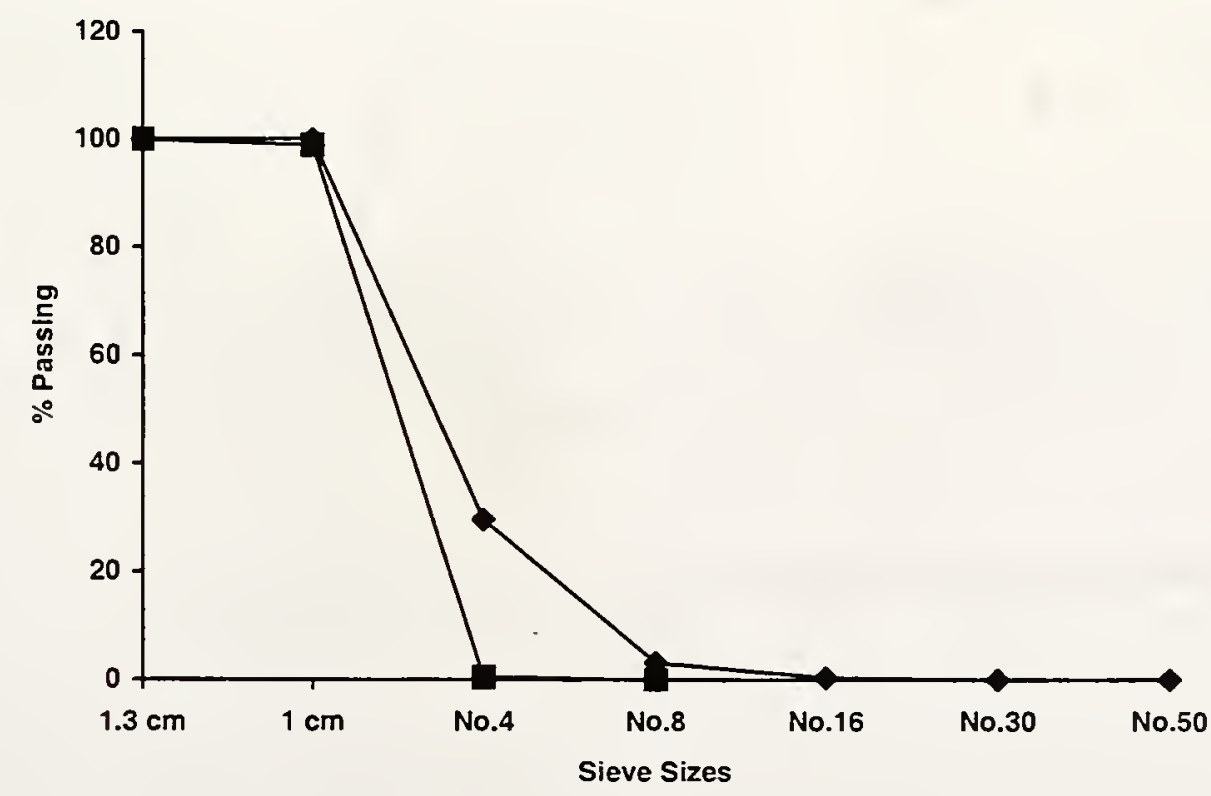

Figure 4.1 Size Distribution for Lightweight Aggregates 


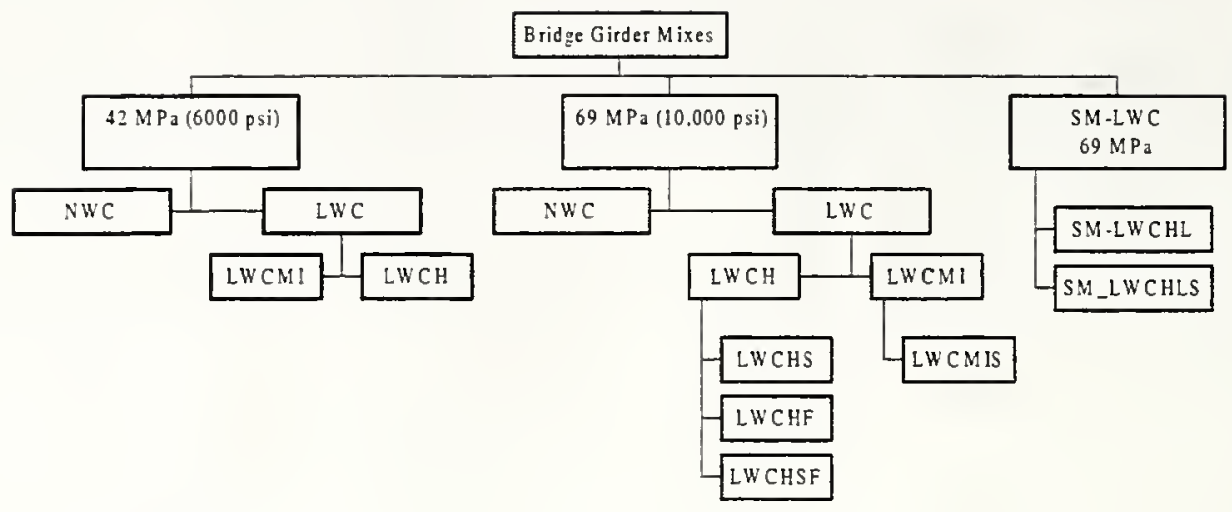

Figure 4.2 Mixture Development Program of Concrete for Use in Bridge Girders

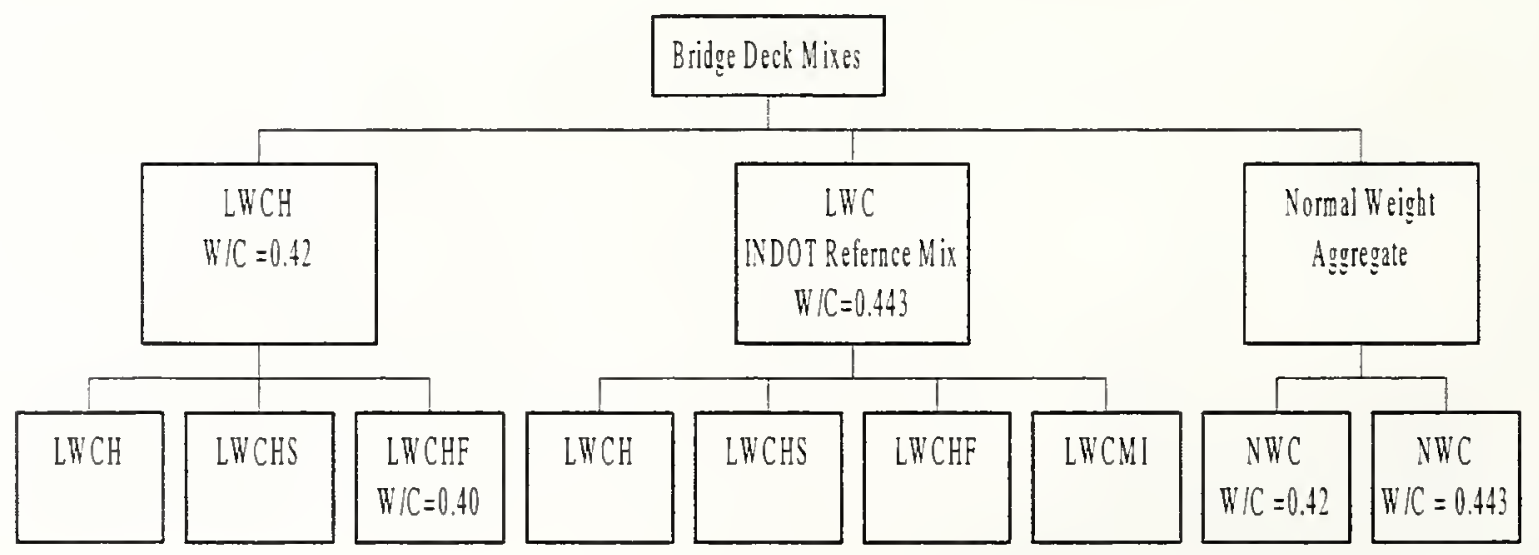

Figure 4.3 Mixture Development Program of Concrete for Use in Bridge Decks 


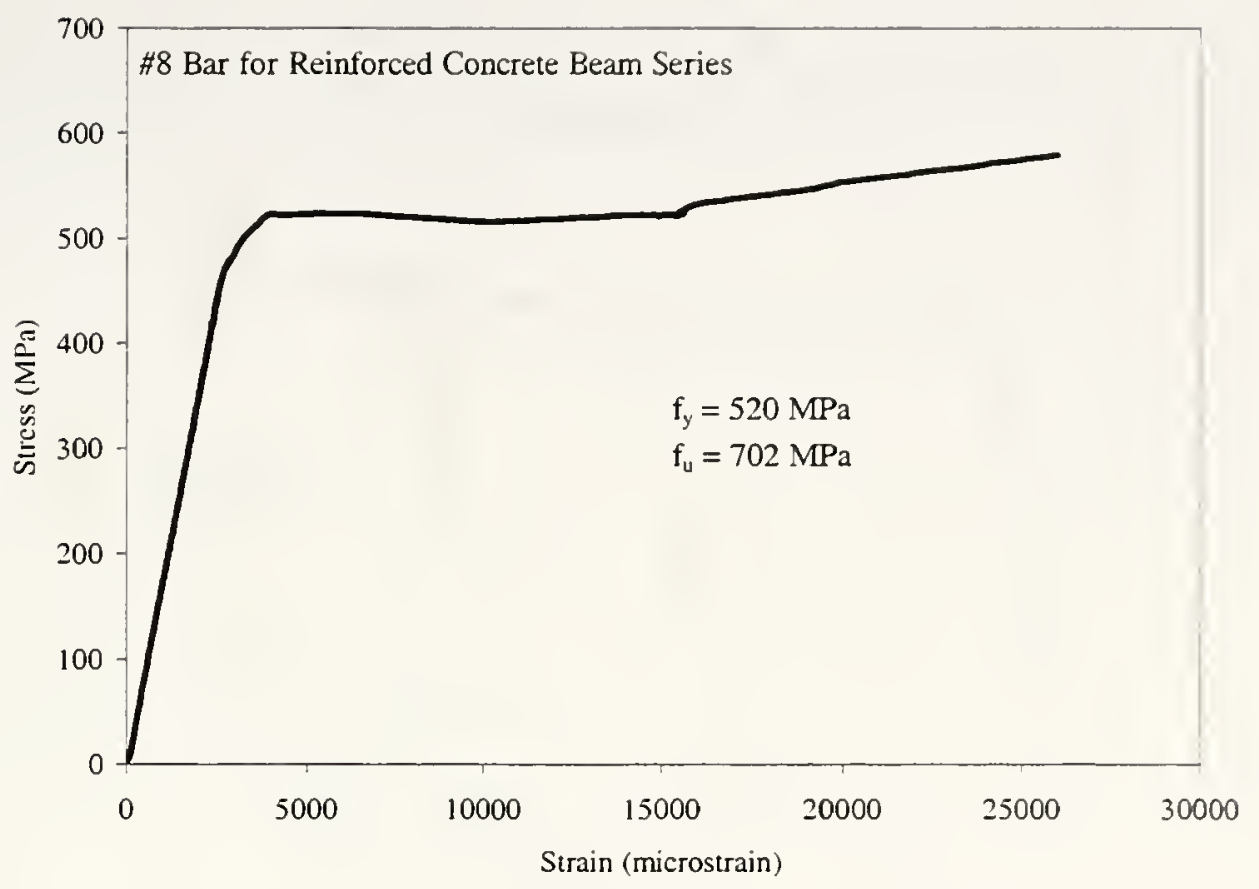

Figure 4.4 Stress-Strain Relationship for No. 8 Bars in Reinforced Concrete Series

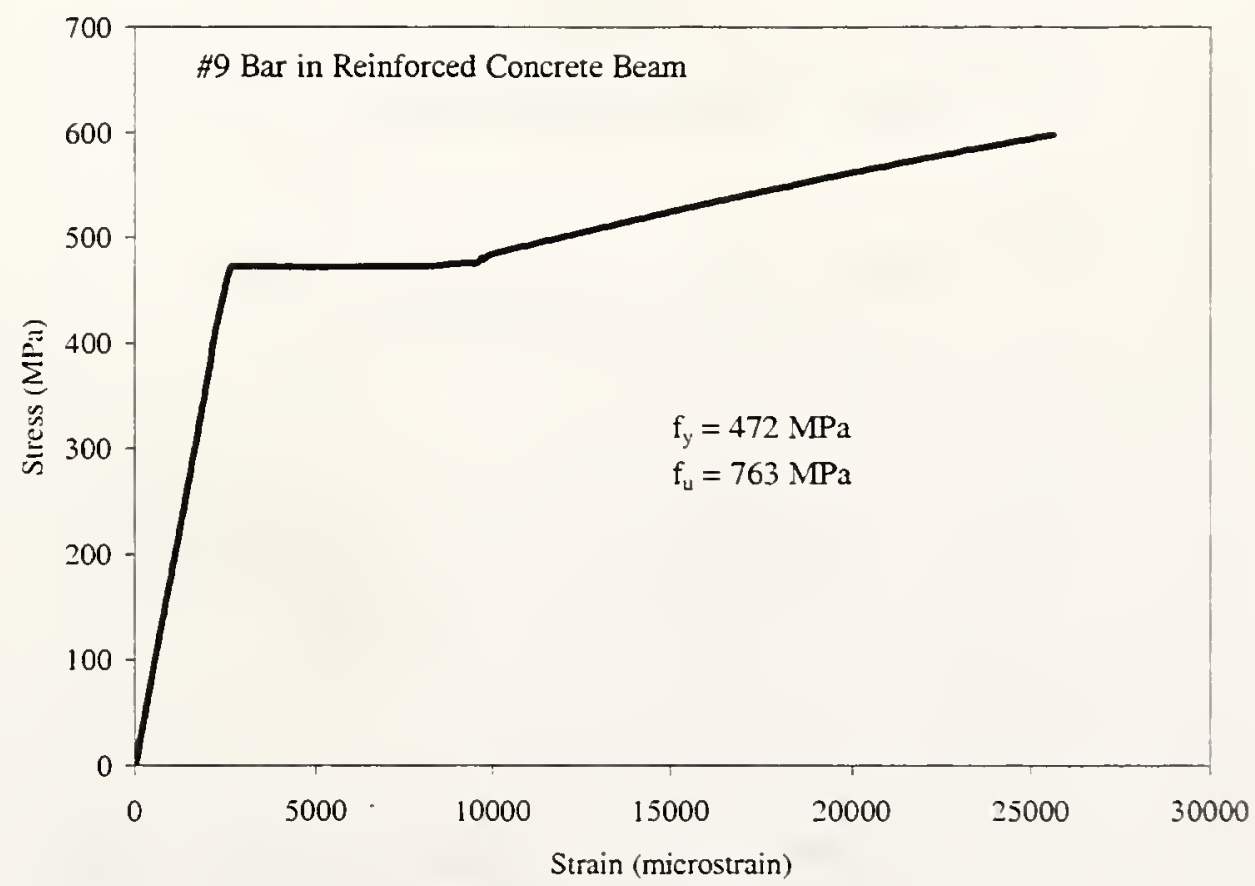

Figure 4.5 Stress-Strain Relationship for No. 9 Bars in Reinforced Concrete Series 


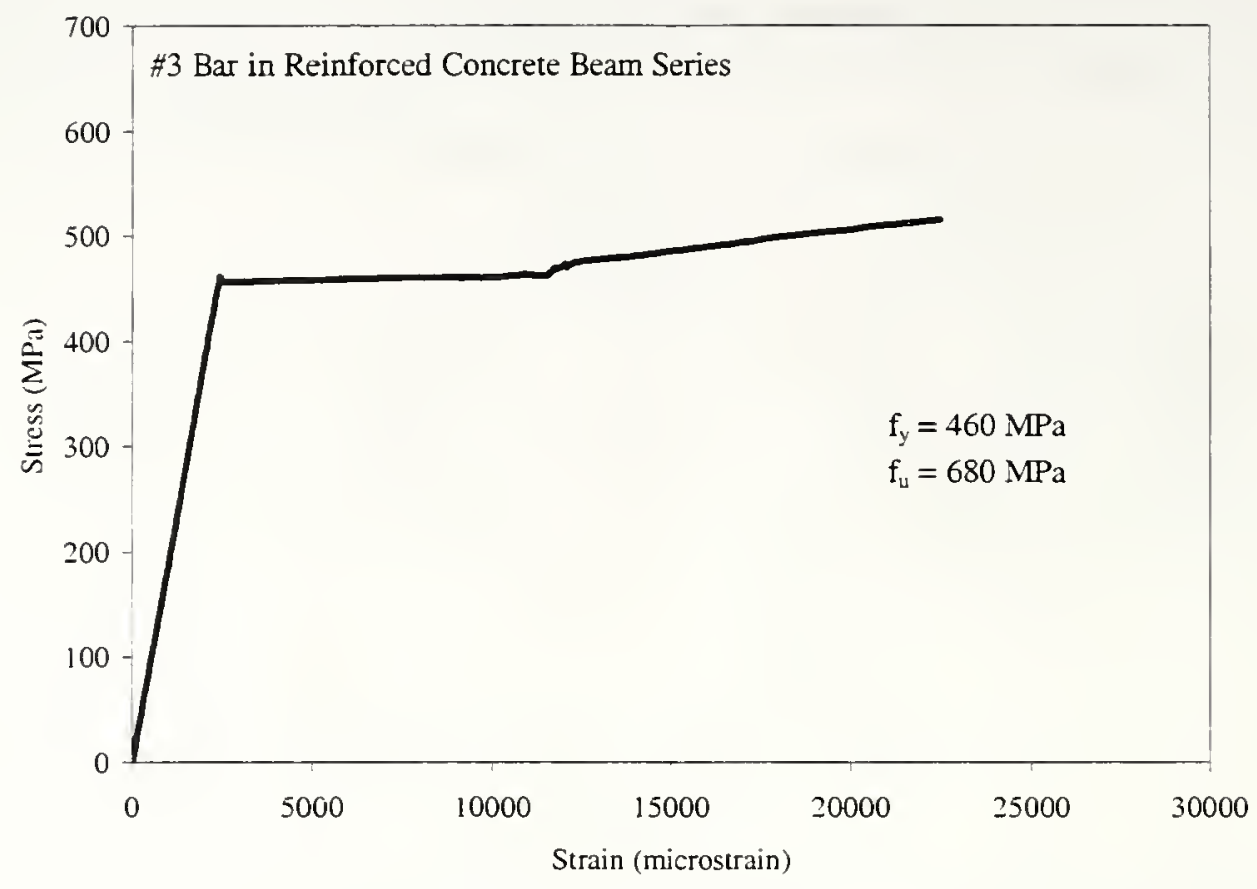

Figure 4.6 Stress-Strain Relationship for No. 3 Stimups in Reinforced Concrete Series

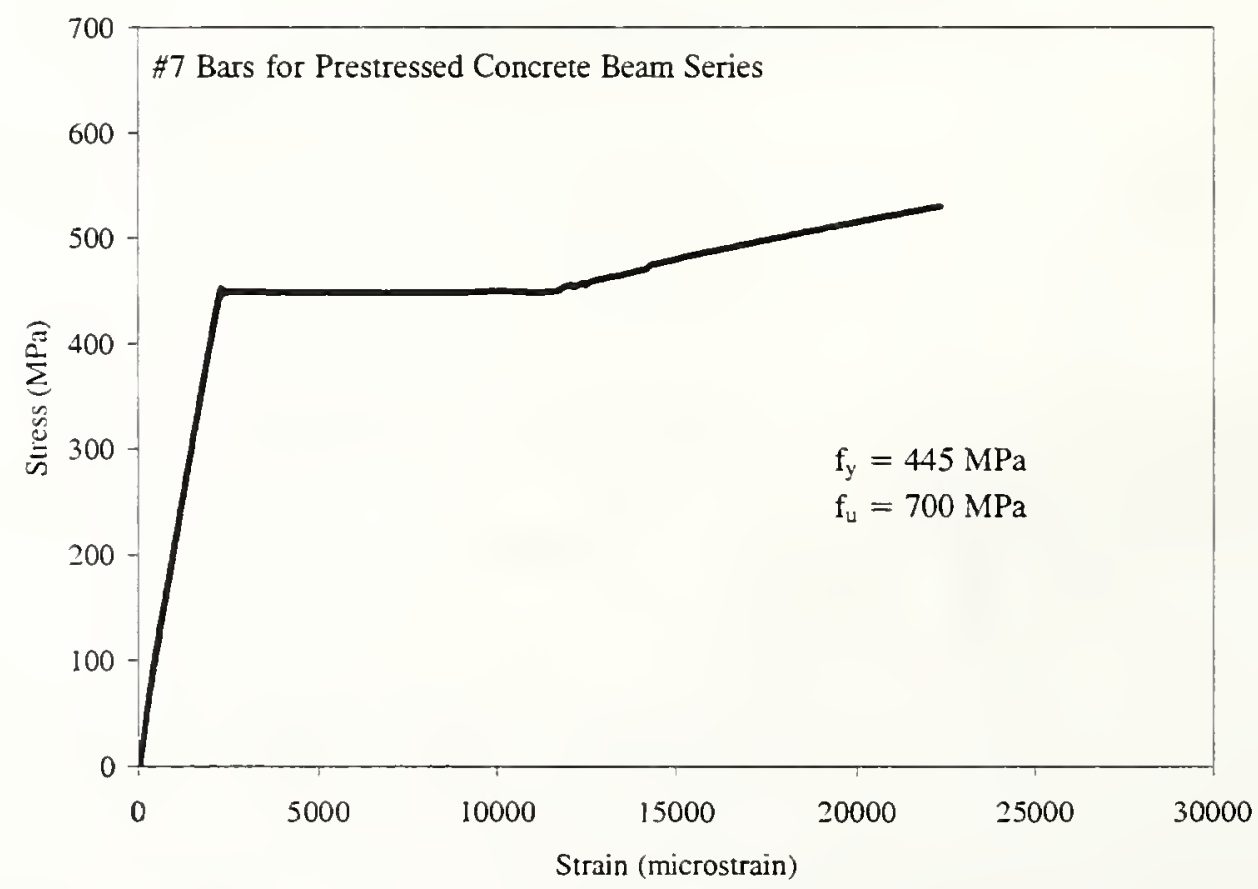

Figure 4.7 Stress-Strain relationship for No. 7 Bars in Prestressed Concrete Series 


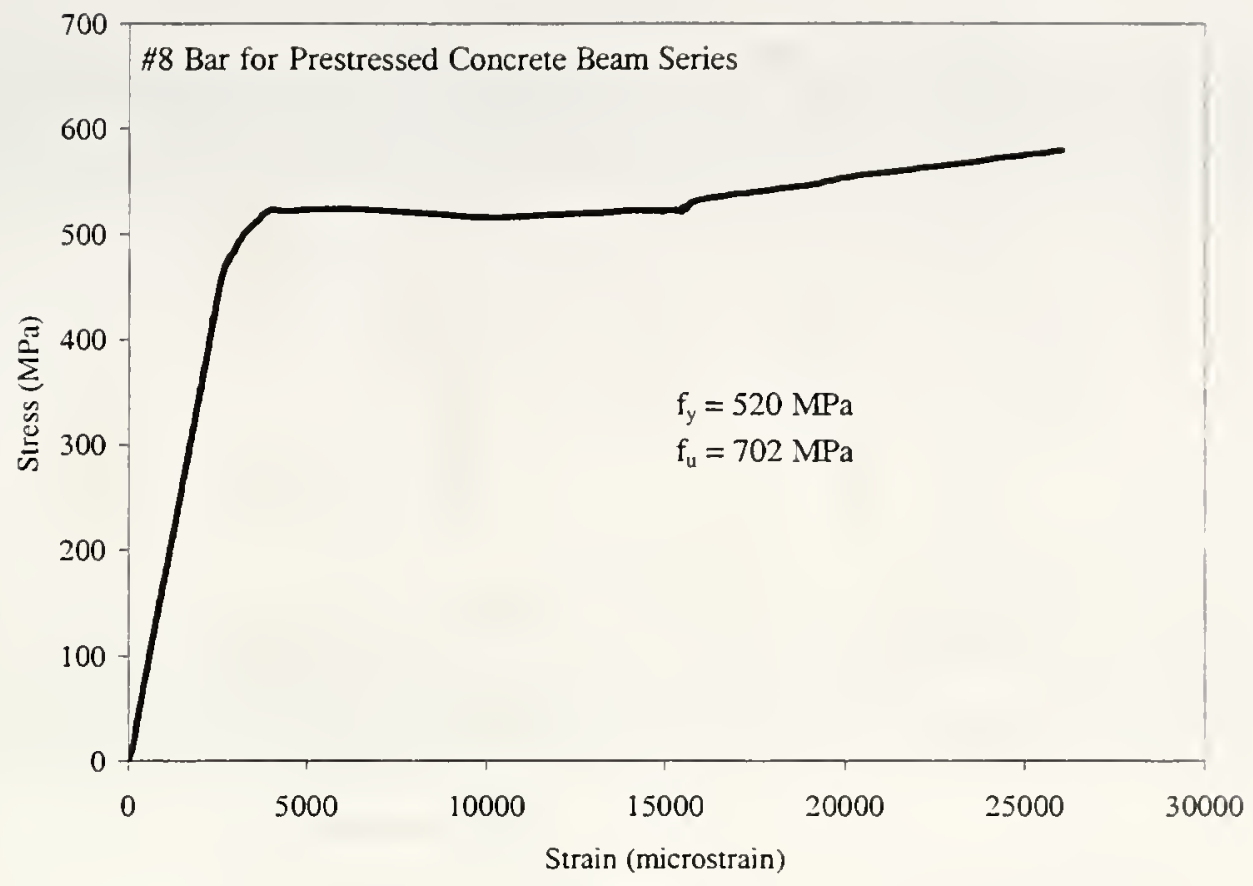

Figure 4.8 Stress-Strain Relationship for No. 8 Bars in Prestressed Concrete Series

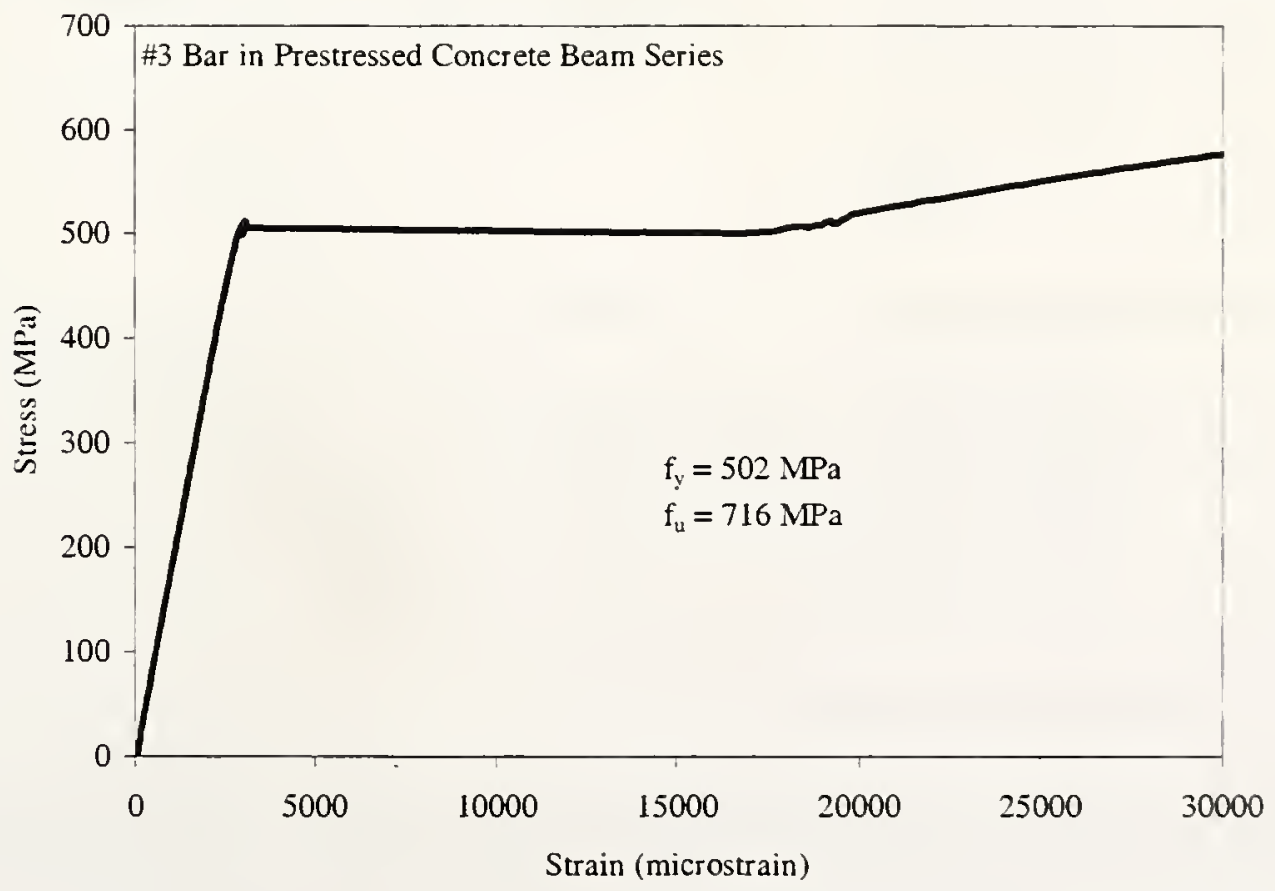

Figure 4.9 Stress-Strain relationship for No. 3 Stirrups in Prestressed Concrete Series 


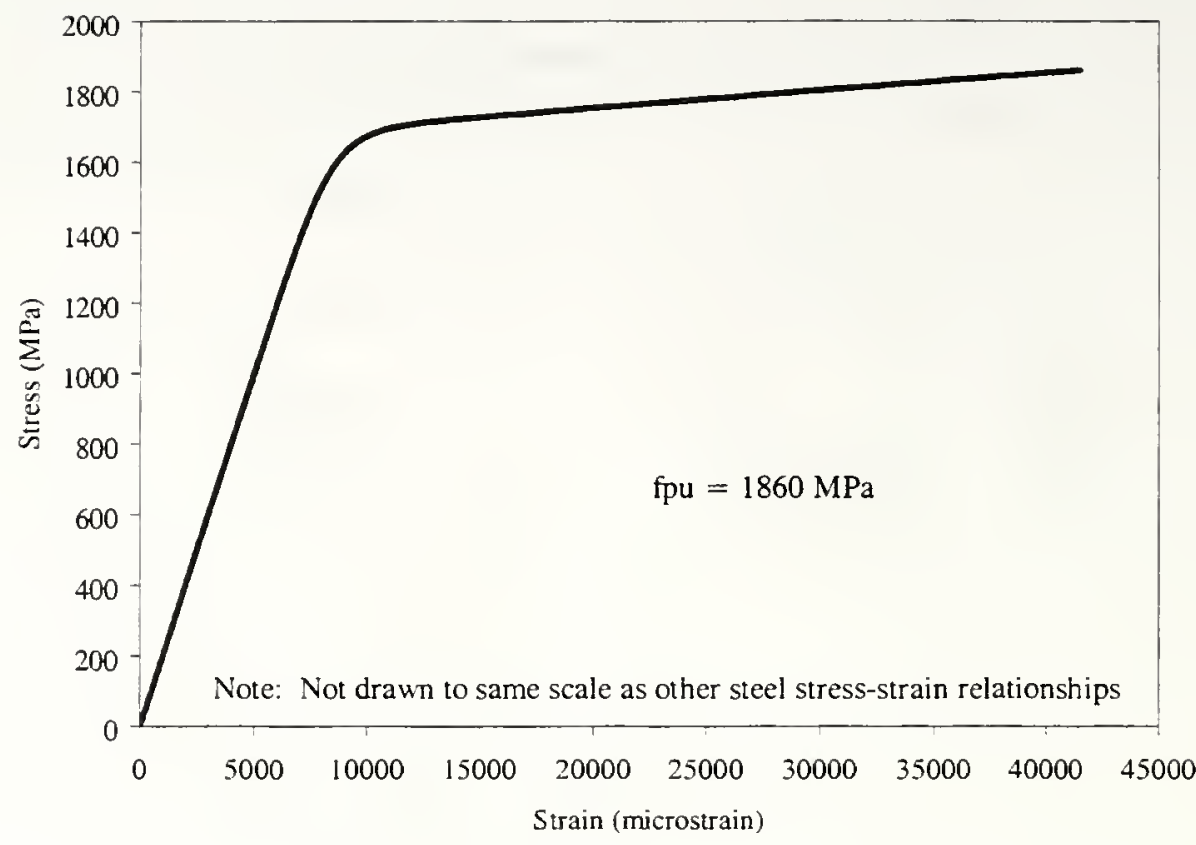

Figure 4.10 Idealized Stress-Strain Relationship for $1 / 2$ Inch Special Low-Relaxation 7-Wire Prestressing Strand 


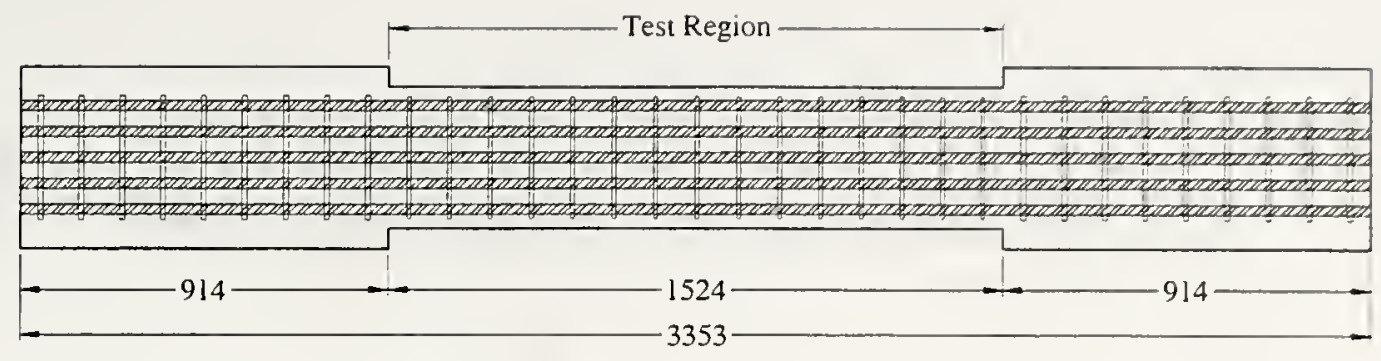

PLAN

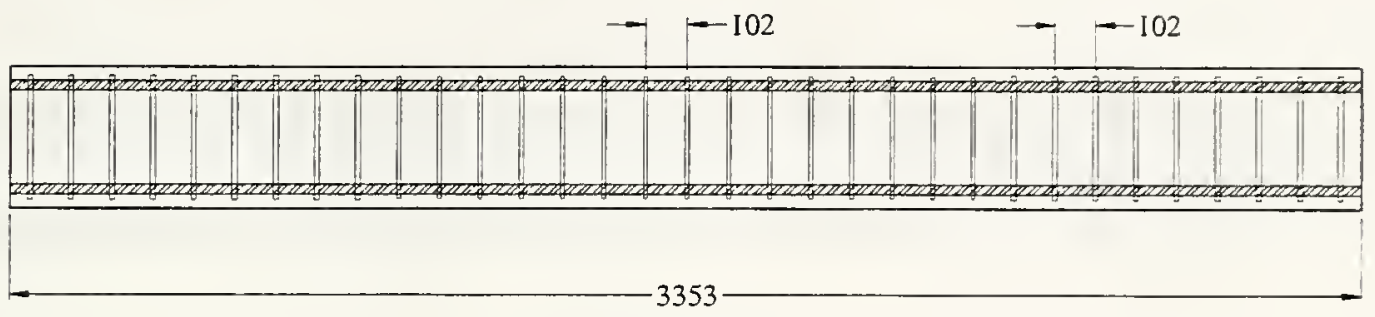

ELEVATION

\section{CROSS SECTION \\ TEST REGION}

CROSS SECTION NON-TEST REGION
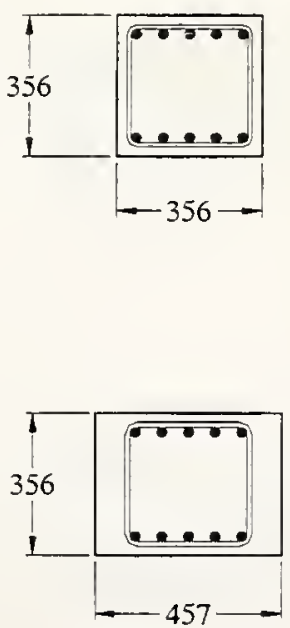

Top Longitudinal Reinforcement: 5 No. 8 Deformed Bars

Stimups:

No. 3 Deformed Bars

Bottom Longitudinal Reinforcement: 5 No. 8 Deformed Bars

Clear Cover to Stimups:

Top and Bottom: 25

Sides: 25

Top Longitudinal Reinforcement: 5 No. 8 Deformed Bars

Stimups:

No. 4 Deformed Bars

Bottom Longitudinal Reinforcement: 5 No. 8 Deformed Bars

Clear Cover to Stirrups:

Top and Bottom: 22

Sides: 73

Dimensions shown in millimeters.

Figure 4.11 Nominal Dimensions and Reinforcement Details for Specimens 1-NWLA and 5-LWLA 


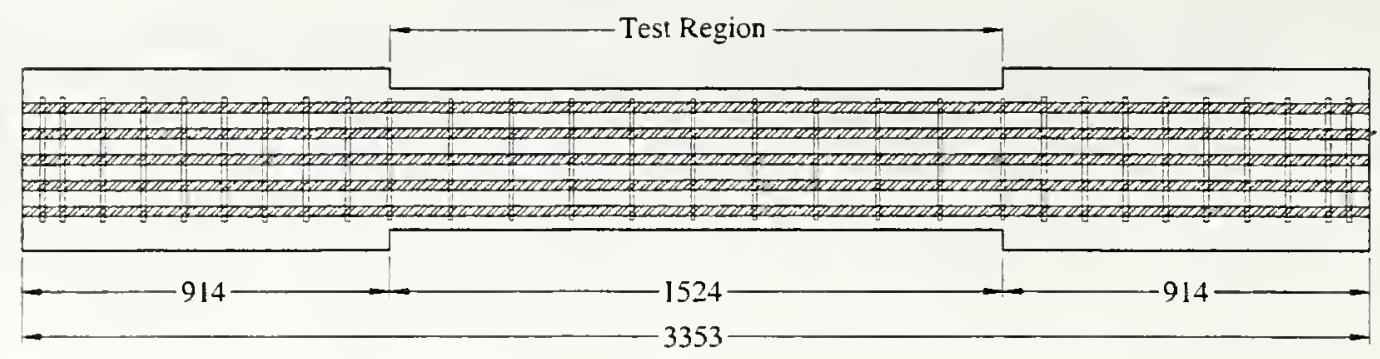

PLAN

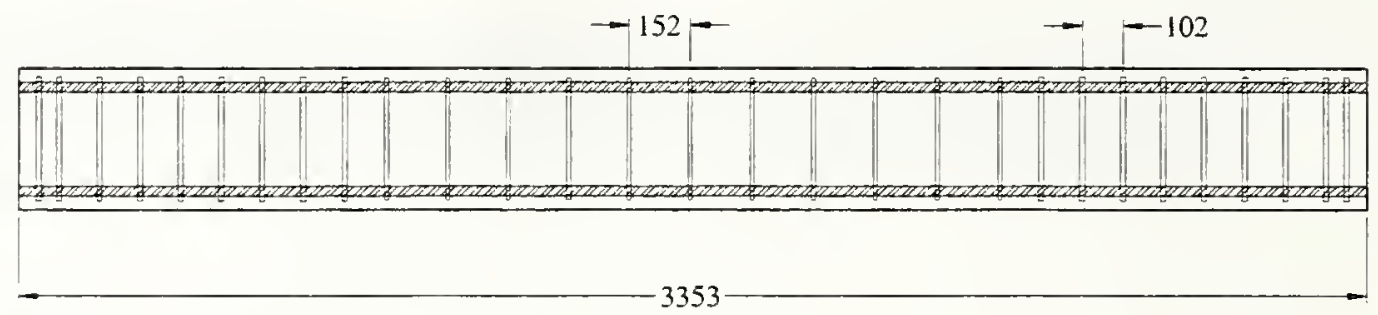

\section{ELEVATION}

CROSS SECTION TEST REGION

CROSS SECTION NON-TEST REGION
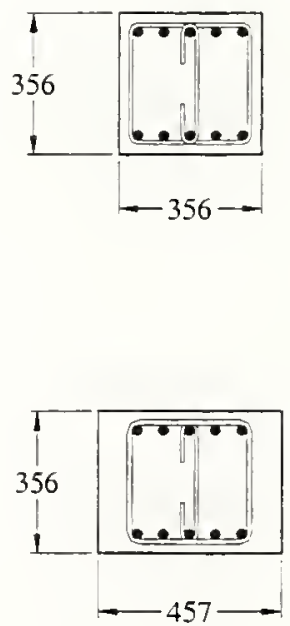

Top Longitudinal Reinforcement: 5 No. 8 Deformed Bars

Stimups:

No. 3 Deformed Bars

Bottom Longitudinal Reinforcement: 5 No. 8 Deformed Bars

Clear Cover to Stirrups:

Top and Bottom: 25

Sides: 25

Top Longitudinal Reinforcement: 5 No. 8 Deformed Bars

Stimups:

No. 4 Deformed Bars

Bottom Longitudinal Reinforcement: 5 No. 8 Deformed Bars

Clear Cover to Stirnups:

Top and Bottom: 22

Sides: 73

Dimensions shown in millimeters.

\section{Figure 4.12 Nominal Dimensions and Reinforcement Details for Specimens 2-NWLB} and 6-LWLB 


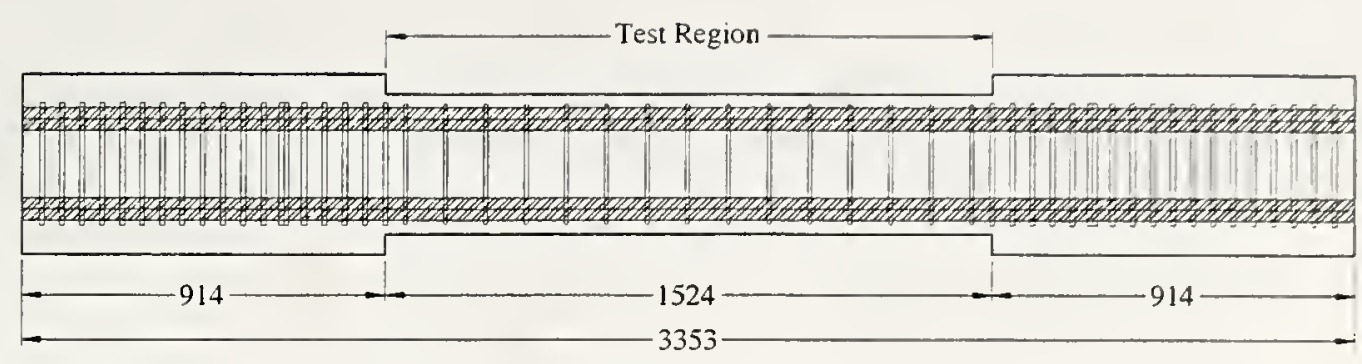

PLAN

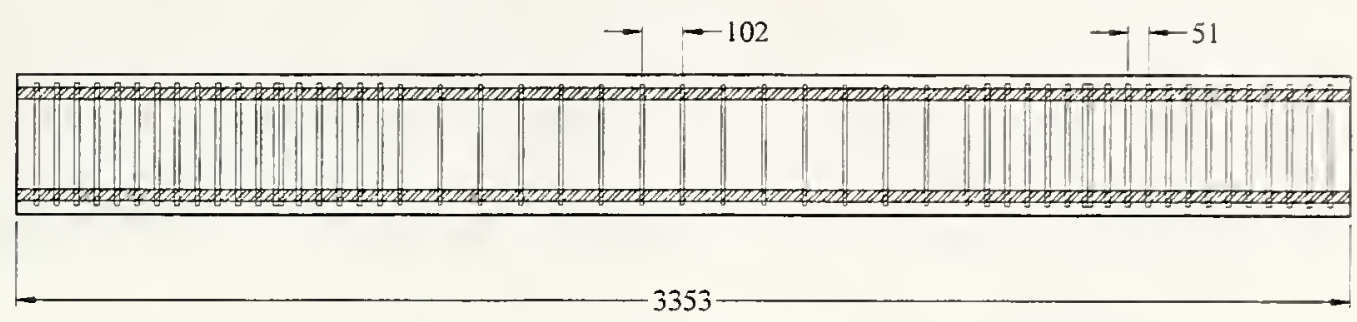

ELEVATION

CROSS SECTION

TEST REGION

CROSS SECTION NON-TEST REGION
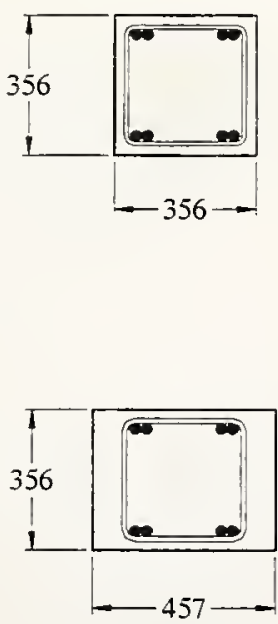

Top Longitudinal Reinforcement:

4 No. 9 Deformed Bars

Stirups:

No. 3 Deformed Bars

Bottom Longitudinal Reinforcement:

4 No. 9 Deformed Bars

Clear Cover to Stimups:

Top and Bottom: 25

Sides: 25

Top Longitudinal Reinforcement:

4 No. 9 Deformed Bars

Stirrups:

No. 4 Deformed Bars

Bottom Longitudinal Reinforcement:

4 No. 9 Deformed Bars

Clear Cover to Stimups:

Top and Bottom: 22

Sides: 73

Dimensions shown in millimeters

Figure 4.13 Nominal Dimensions and Reinforcement Details for Specimens 3-NWLC and 7-LWLC 


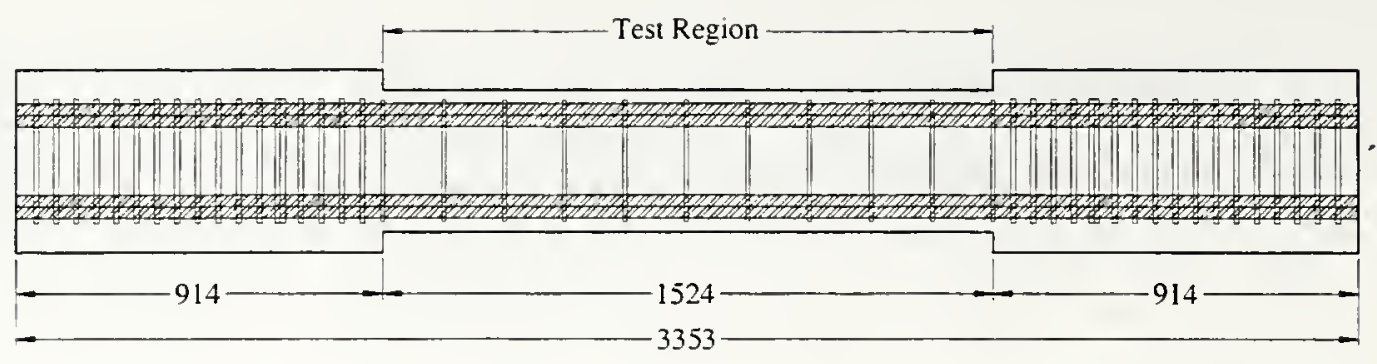

PLAN

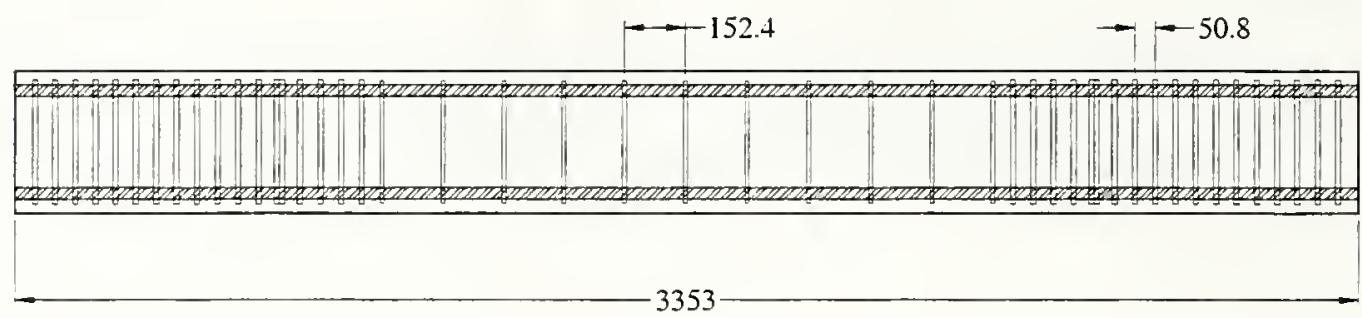

ELEVATION

CROSS SECTION

TEST REGION

CROSS SECTION NON-TEST REGION
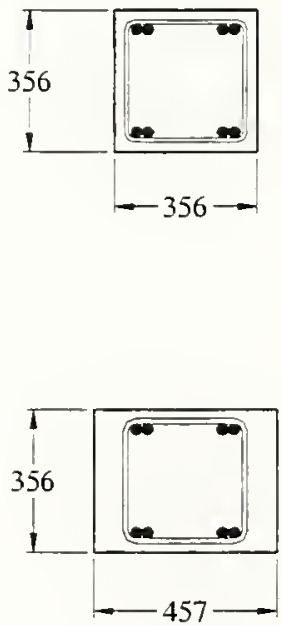

Top Longitudinal Reinforcement: 4 No. 9 Deformed Bars

Stimups:

No. 3 Deformed Bars

Bottom Longitudinal Reinforcement: 4 No. 9 Deformed Bars

Clear Cover to Stirrups:

Top and Bottom: 25

Sides: 25

Top Longitudinal Reinforcement: 4 No. 9 Deformed Bars

Stimups:

No. 4 Deformed Bars

Bottom Longitudinal Reinforcement:

4 No. 9 Deformed Bars

Clear Cover to Stimups:

Top and Bottom: 22

Sides: 73

Dimensions shown in millimeters.

Figure 4.14 Nominal Dimensions and Reinforcement Details for Specimens 4-NWLD, 8-LWLD, 9-NWLD, 10-NWHD, 11-LWHD, and 12-NWHD 
Reinforcing Detail

A

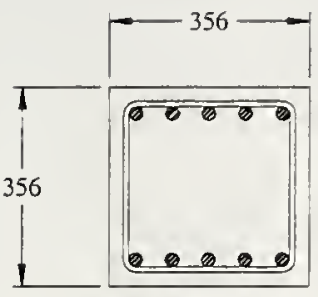

B

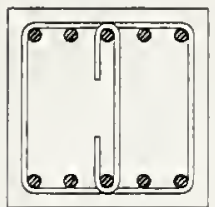

C
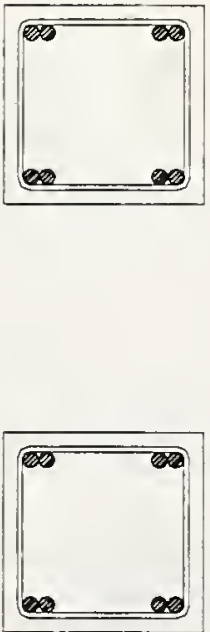

Top Longitudinal Reinforcement: 5 No. 8 Deformed Bars

Stirnups:

No. 3 Double Leg Closed Stirups Spacing $=102$

Bottom Longitudinal Reinforcement: 5 No. 8 Deformed Bars

Top Longitudinal Reinforcement: 5 No. 8 Deformed Bars

Stirrups:

No. 3 Triple Leg Closed Stirups Spacing $=152$

Bottom Longitudinal Reinforcement: 5 No. 8 Deformed Bars

Top Longitudinal Reinforcement: 4 No. 9 Deformed Bars

Stirrups:

No. 3 Double Leg Closed Stirrups Spacing $=102$

Bottom Longitudinal Reinforcement: 4 No. 9 Deformed Bars

Top Longitudinal Reinforcement: 4 No. 9 Deformed Bars

Stirrups:

No. 3 Double Leg Closed Stirrups

Spacing $=152$

Bottom Longitudinal Reinforcement: 4 No. 9 Deformed Bars

Dimensions shown in millimeters.

Figure 4.15 Cross Sections of Reinforcing Details in Test Regions 


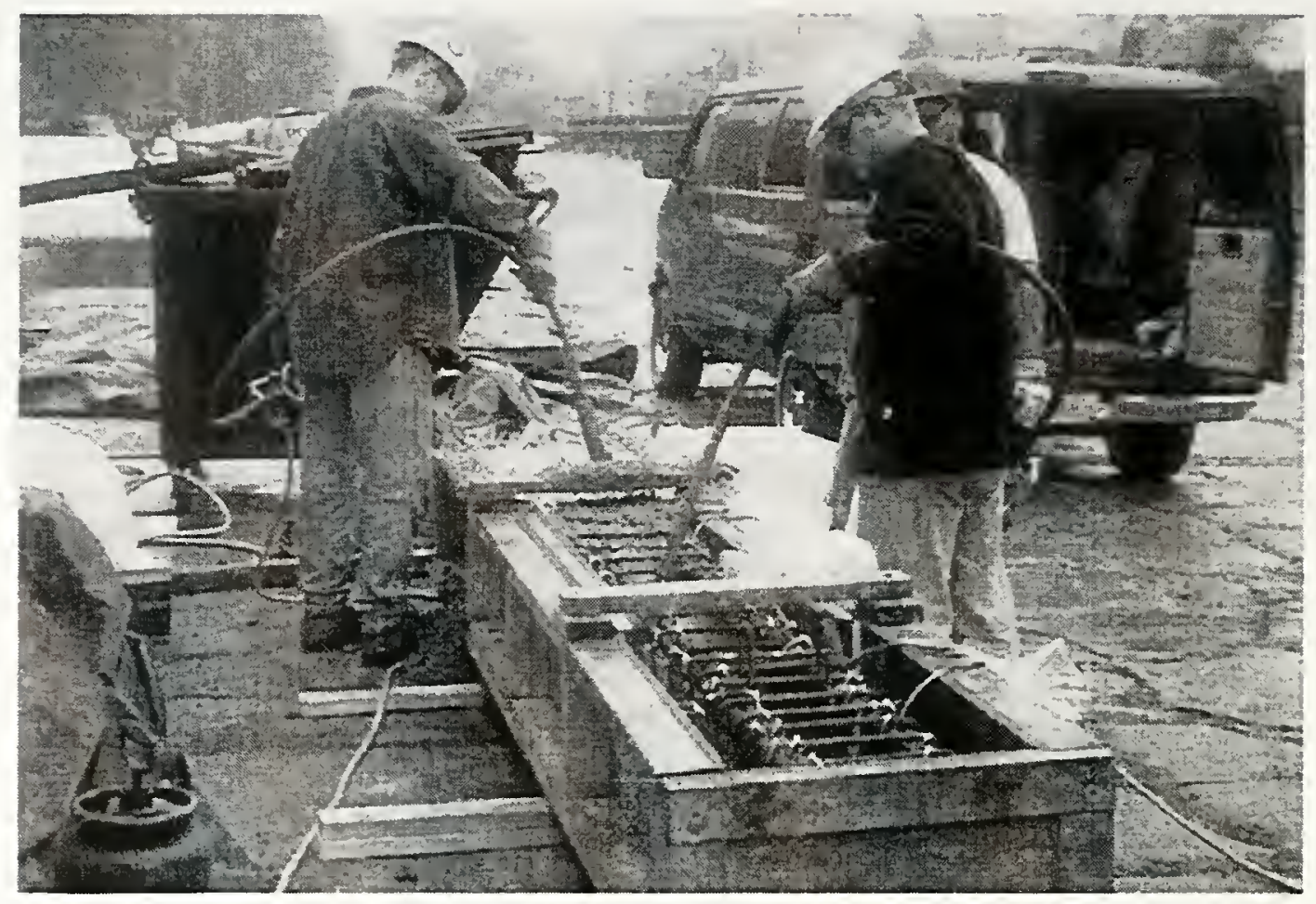

Figure 4.16 Consolidating Concrete with Handheld Vibrators

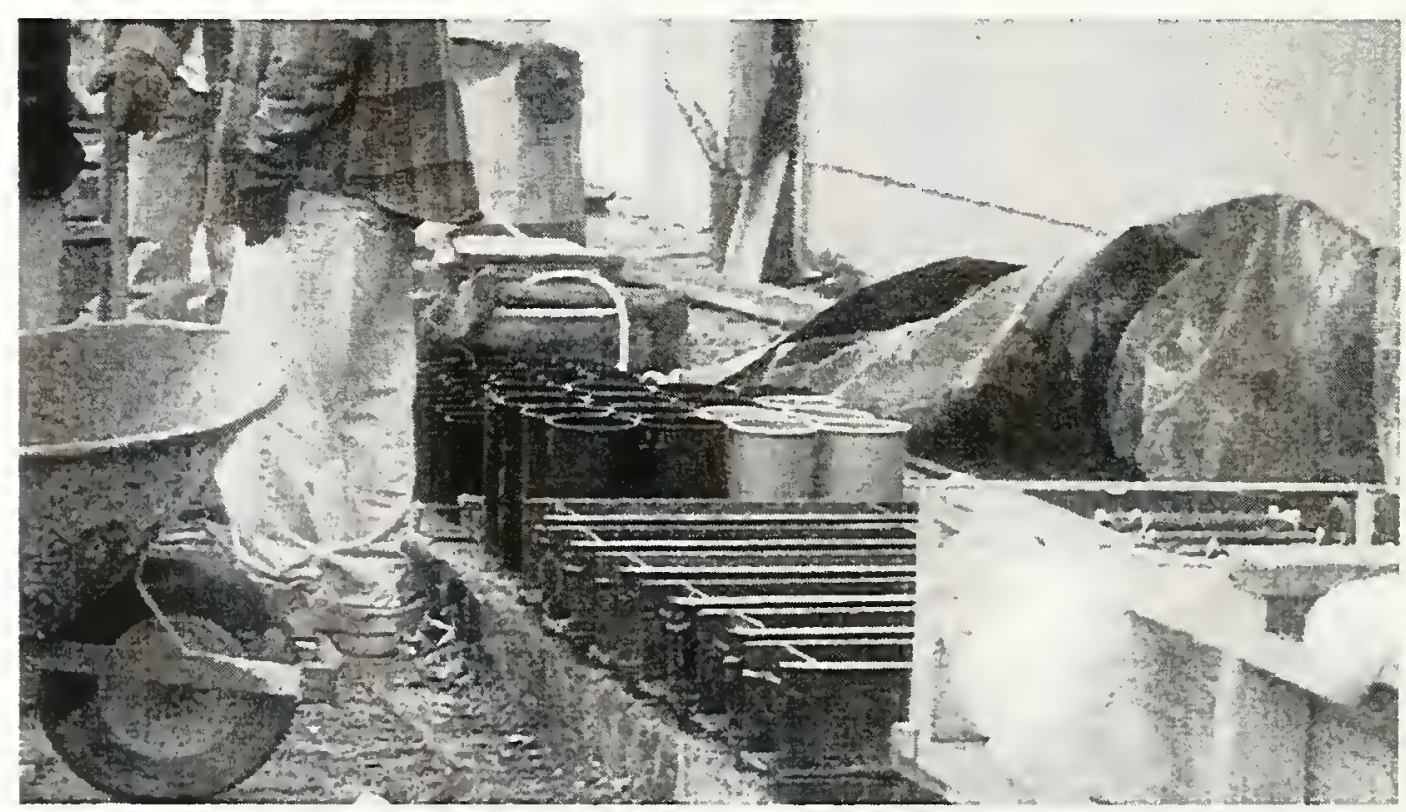

Figure 4.17 Sampling of Concrete at Precast Plant 


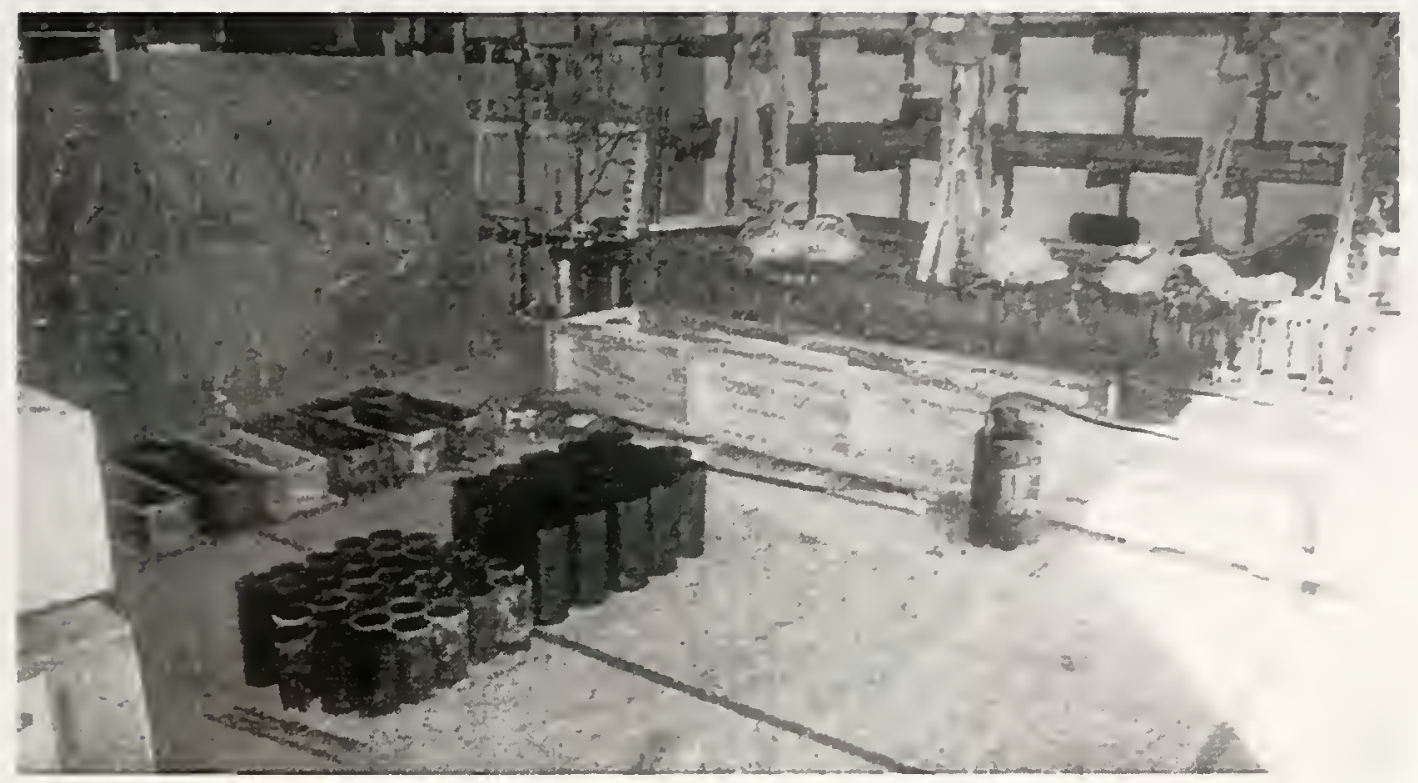

Figure 4.18 Samples and Formwork for Specimen 10-NWHD in Laboratory

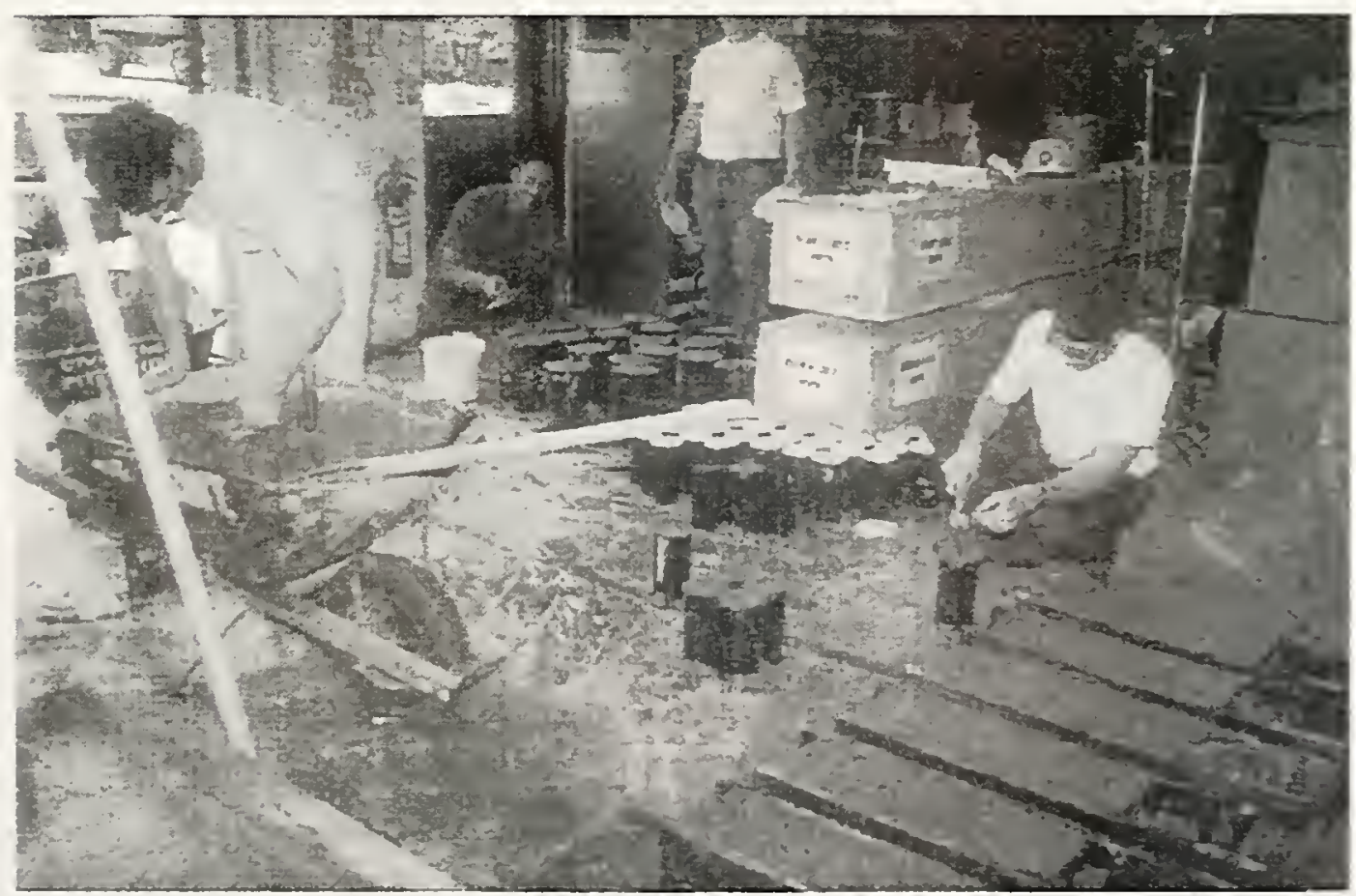

Figure 4.19 Making Test Samples for Specimen 10-NWHD in Laboratory 


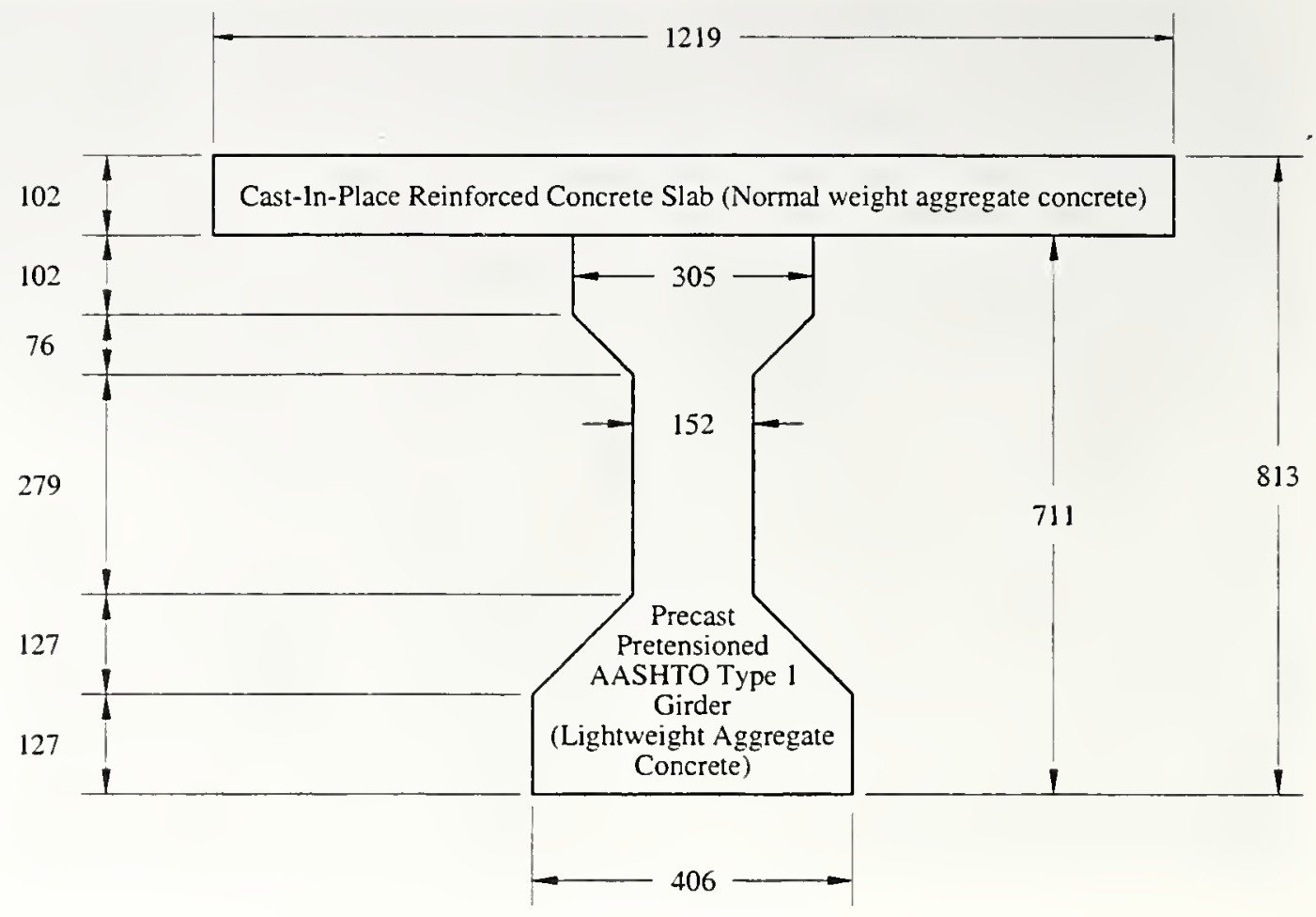

Dimensions shown in millimeters.

Figure 4.20 Cross Sectional Dimensions of Prestressed Concrete Specimens 
Slab Reinforcement: No. 5 @ $127 \mathrm{~mm}$ each way

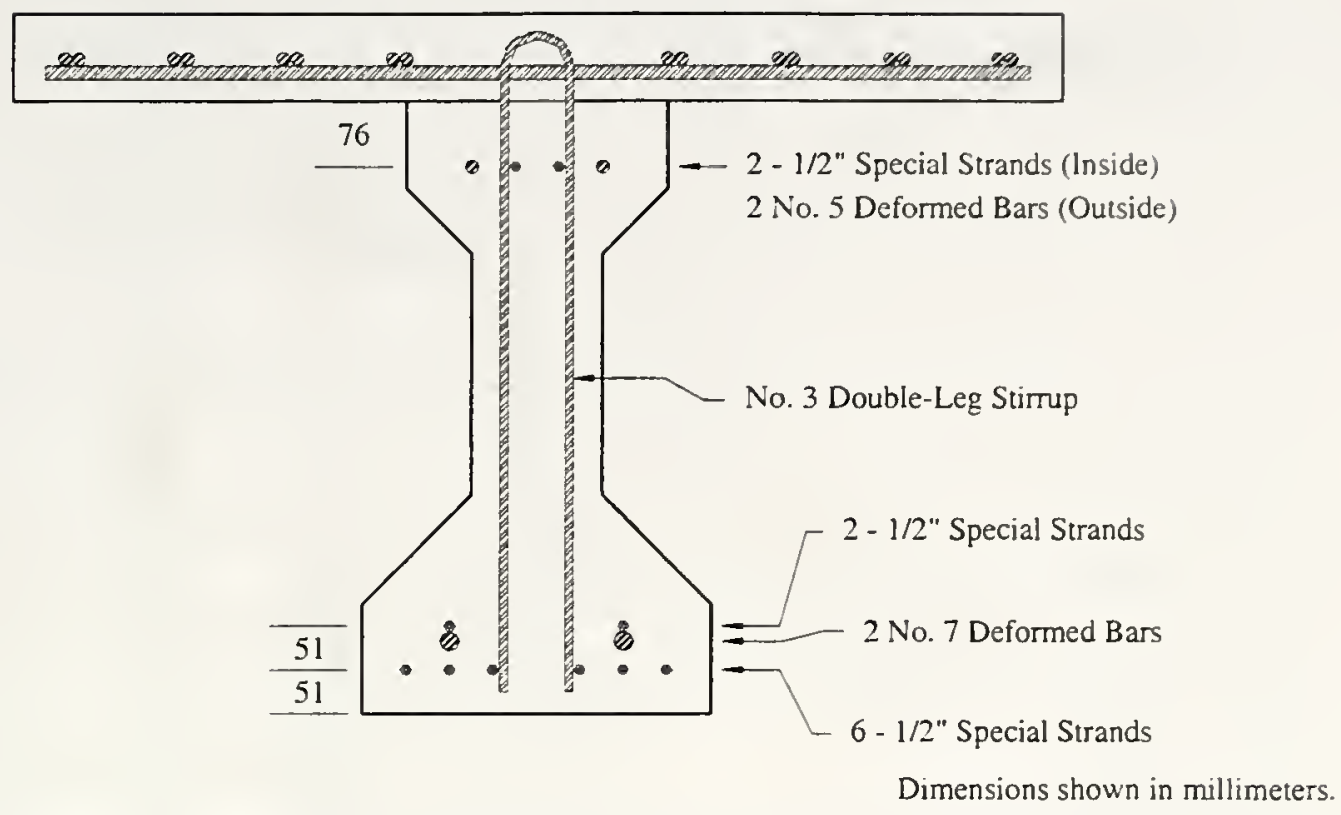

Figure 4.21 Cross Sectional Reinforcement Details of Specimen PC6S at Midspan

Slab Reinforcement: No. 5 @ $127 \mathrm{~mm}$ each way

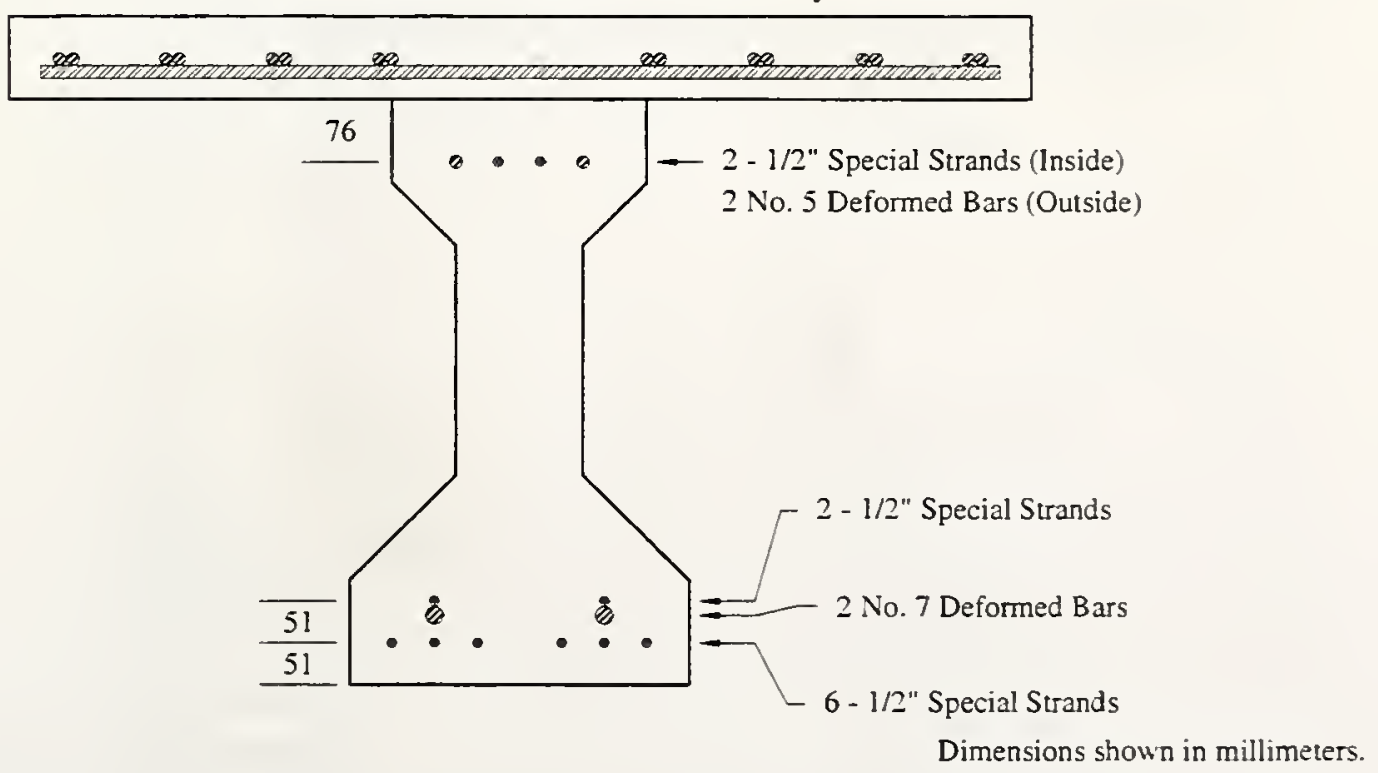

Figure 4.22 Cross Sectional Reinforcement Details of Specimen PC6N at Midspan 
Slab Reinforcement: No. 5 @ 127 mm each way

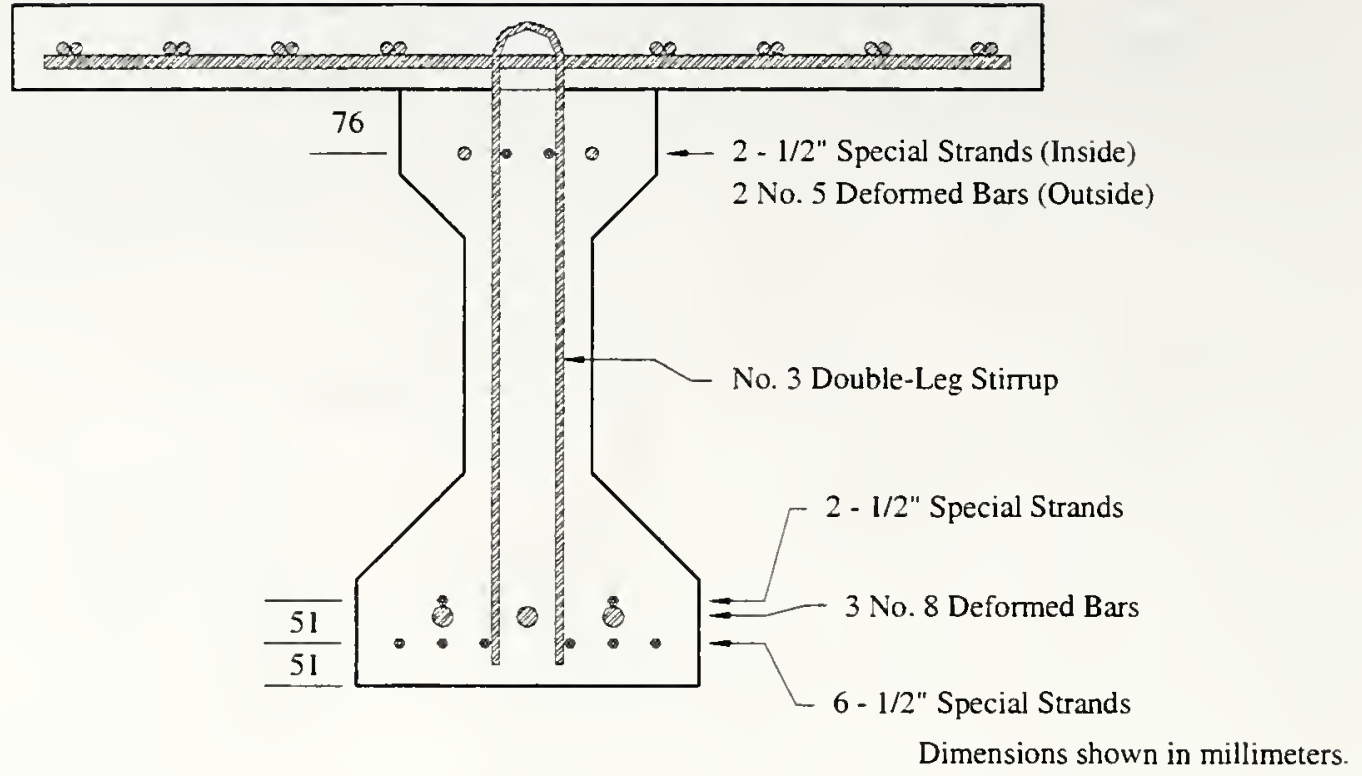

Figure 4.23 Cross Sectional Reinforcement Details of Specimen PC10S at Midspan

Slab Reinforcement: No. 5 @ 127 mm each way

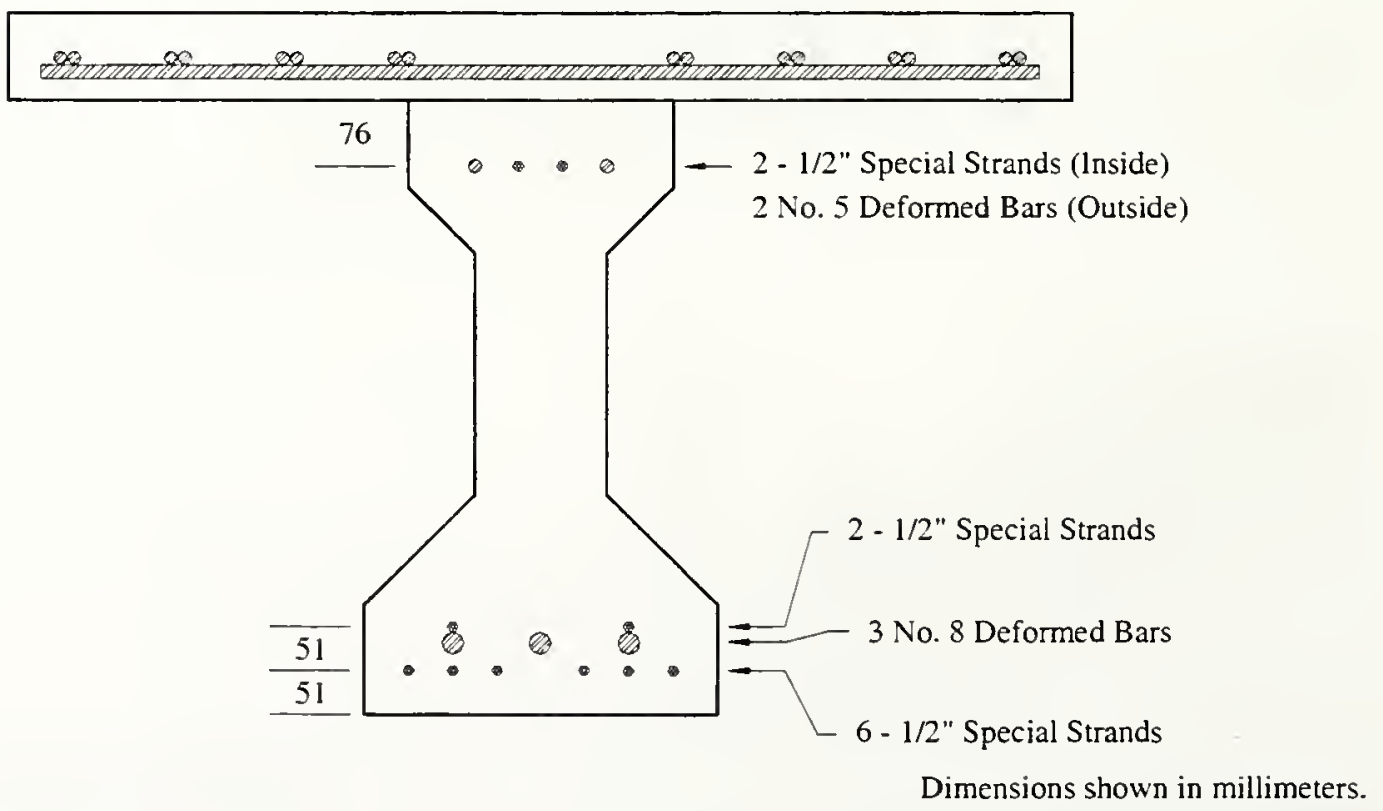

Figure 4.24 Cross Sectional Reinforcement Details of Specimen PC10N at Midspan 


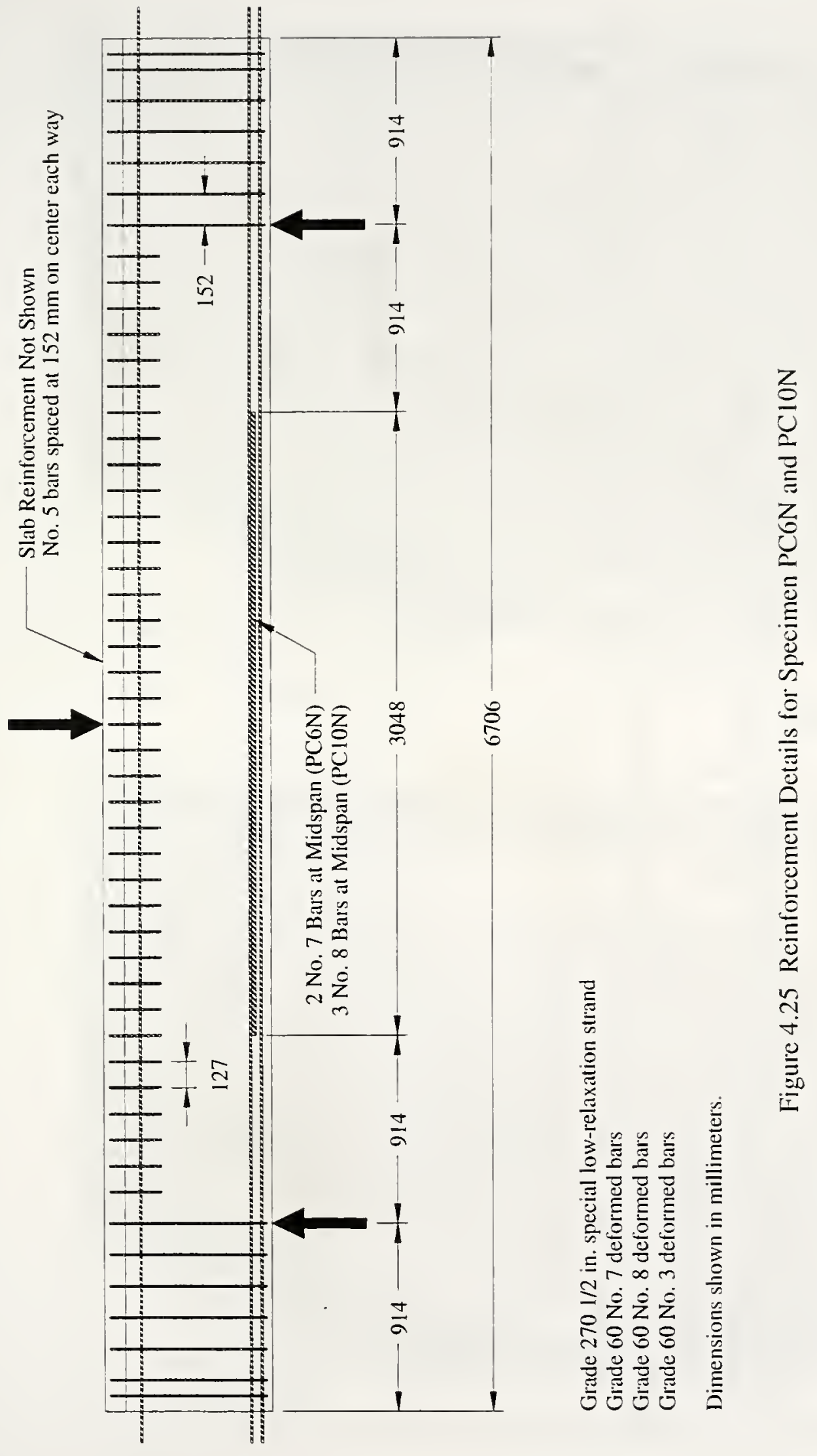




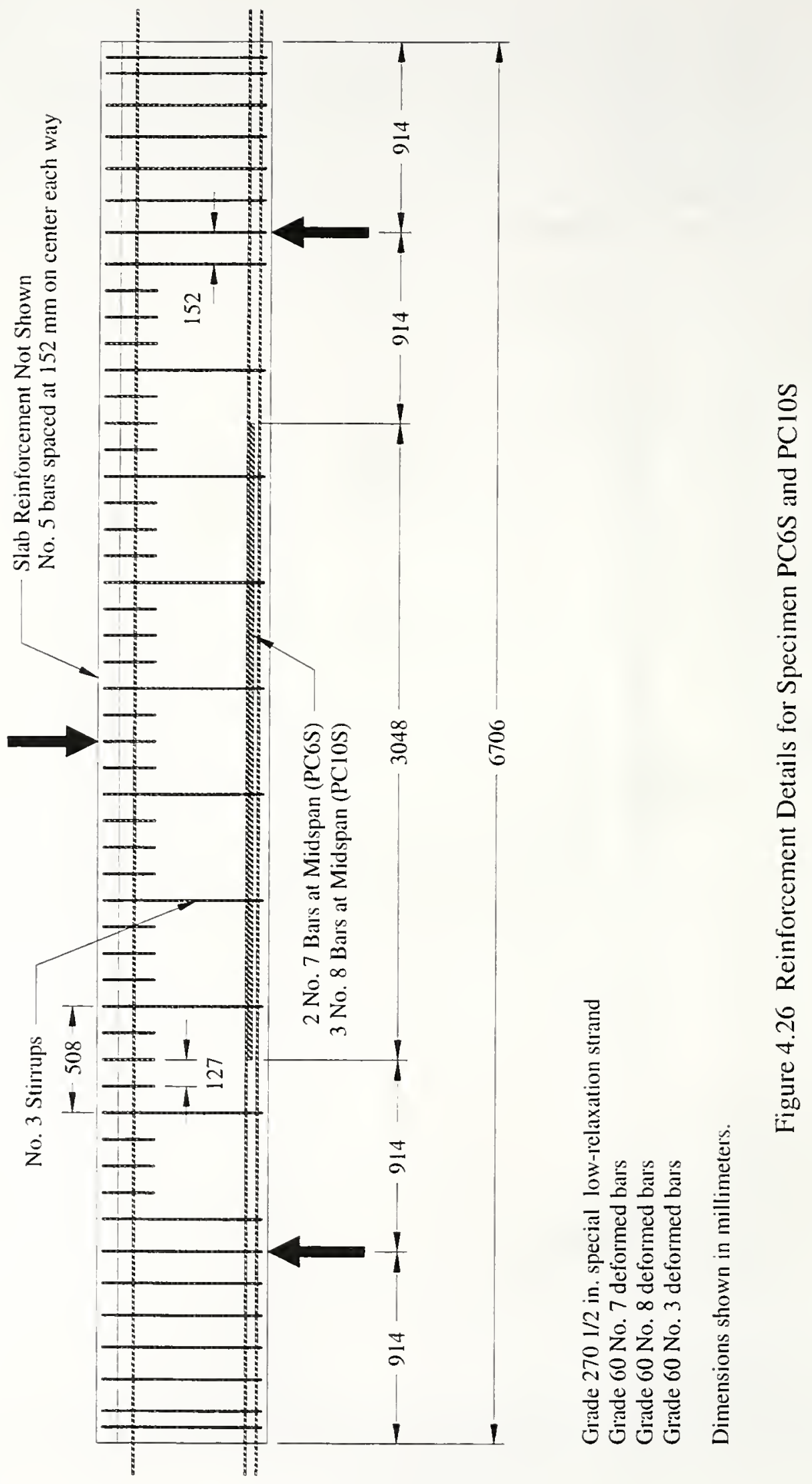




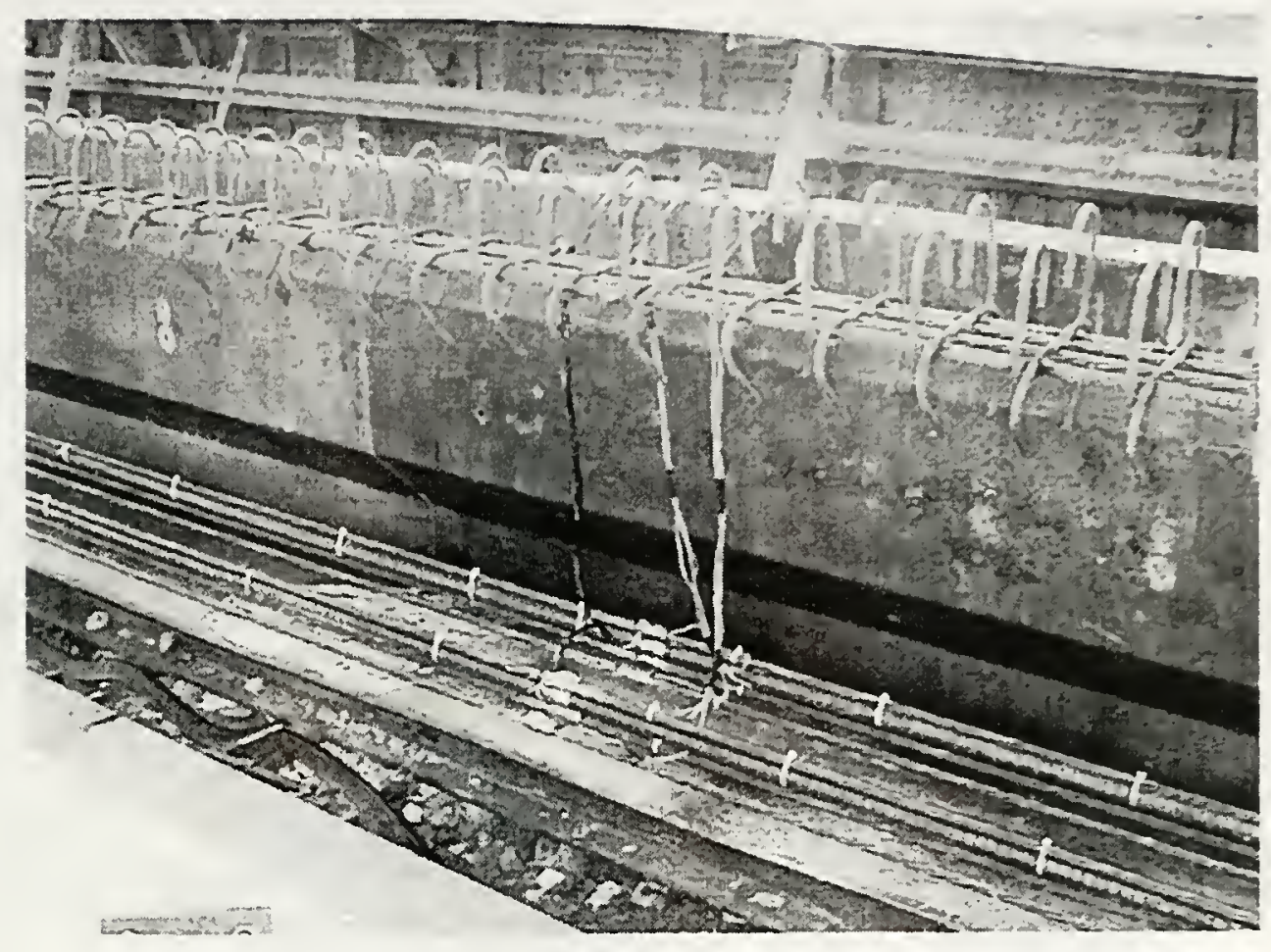

Figure 4.27 Specimen PC6N Prior to Casting at the Precast Plant

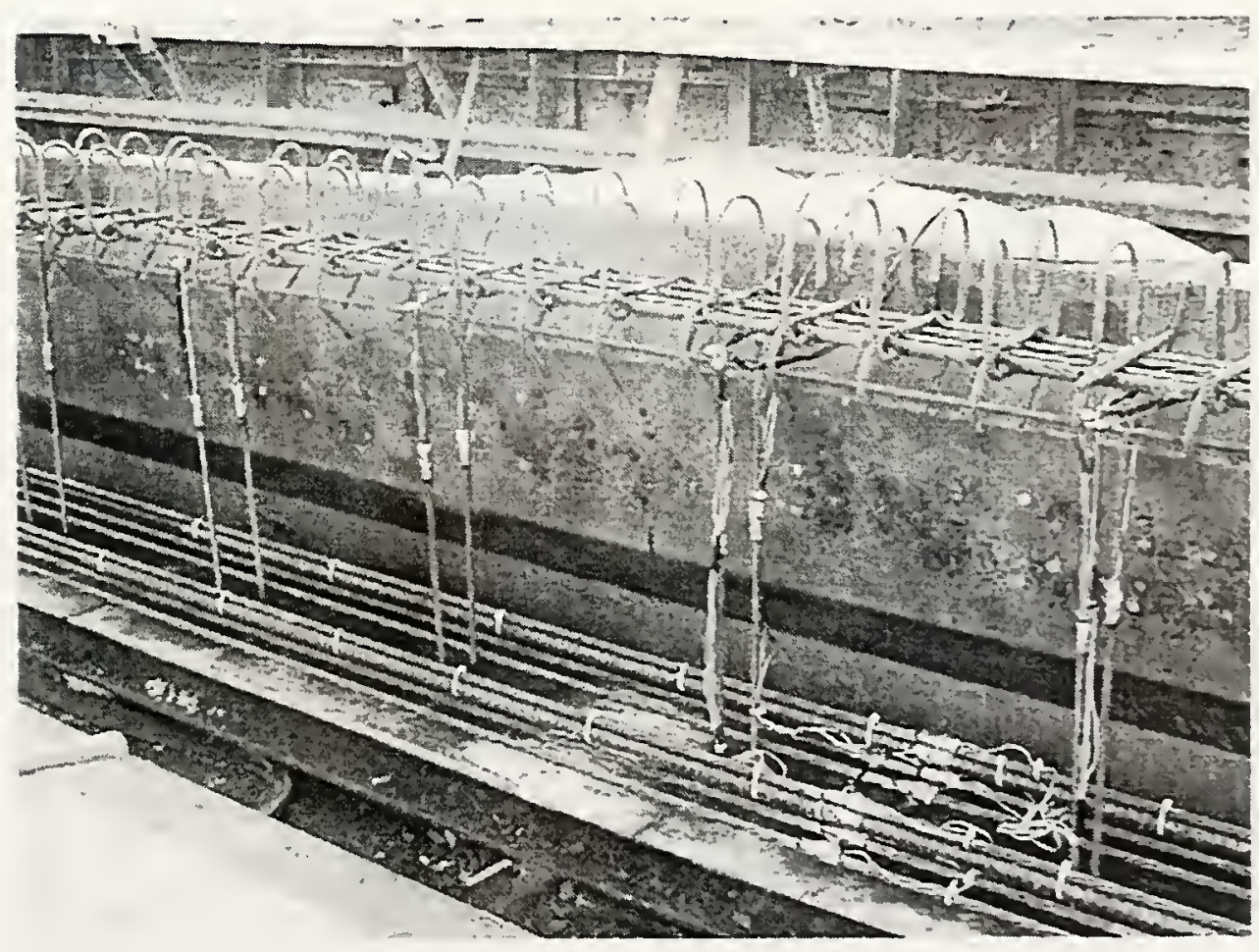

Figure 4.28 Specimen PC6S Prior to Casting at the Precast Plant 


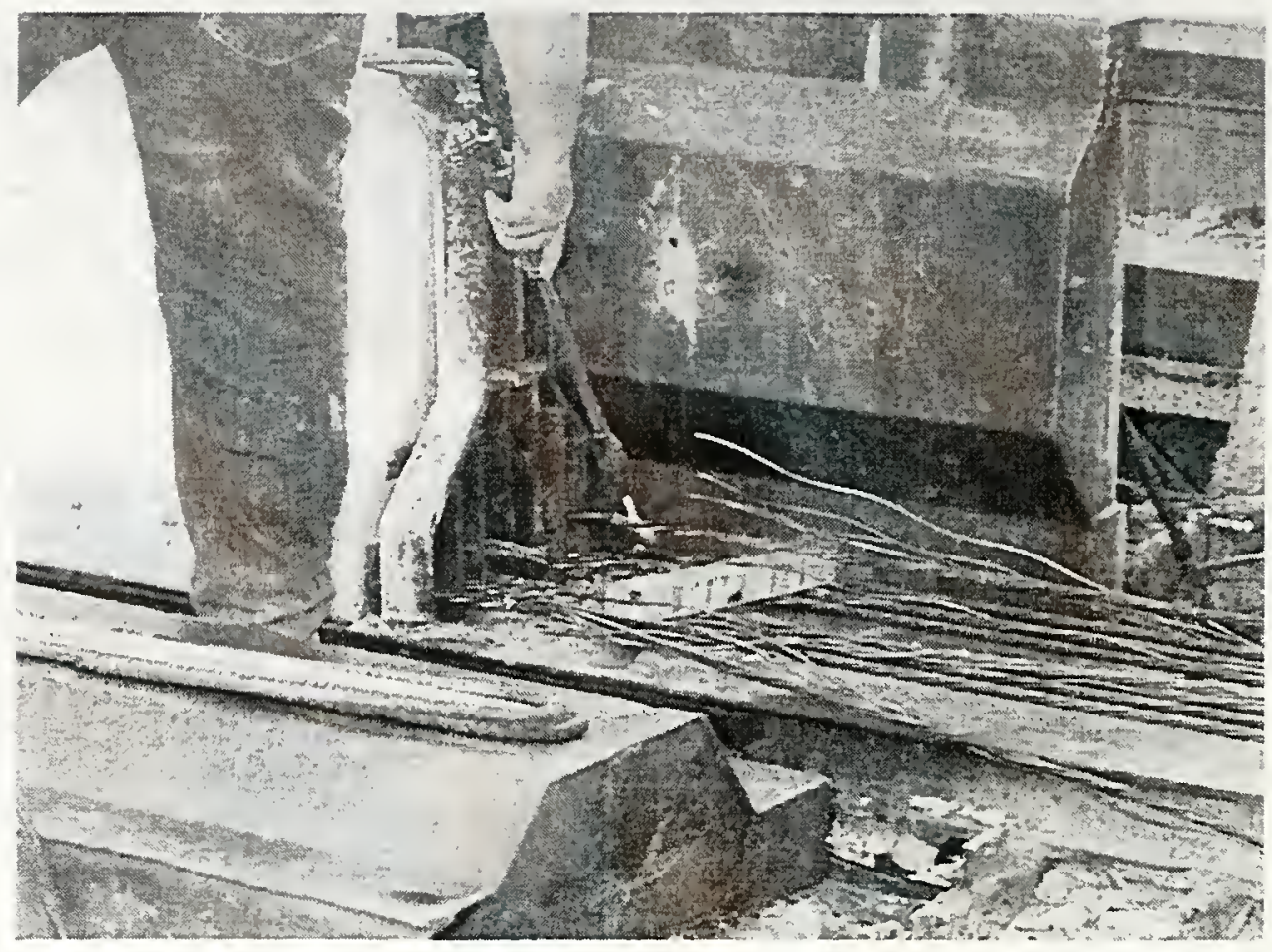

Figure 4.29 Flame-Cutting Strands to Release Prestress Force 

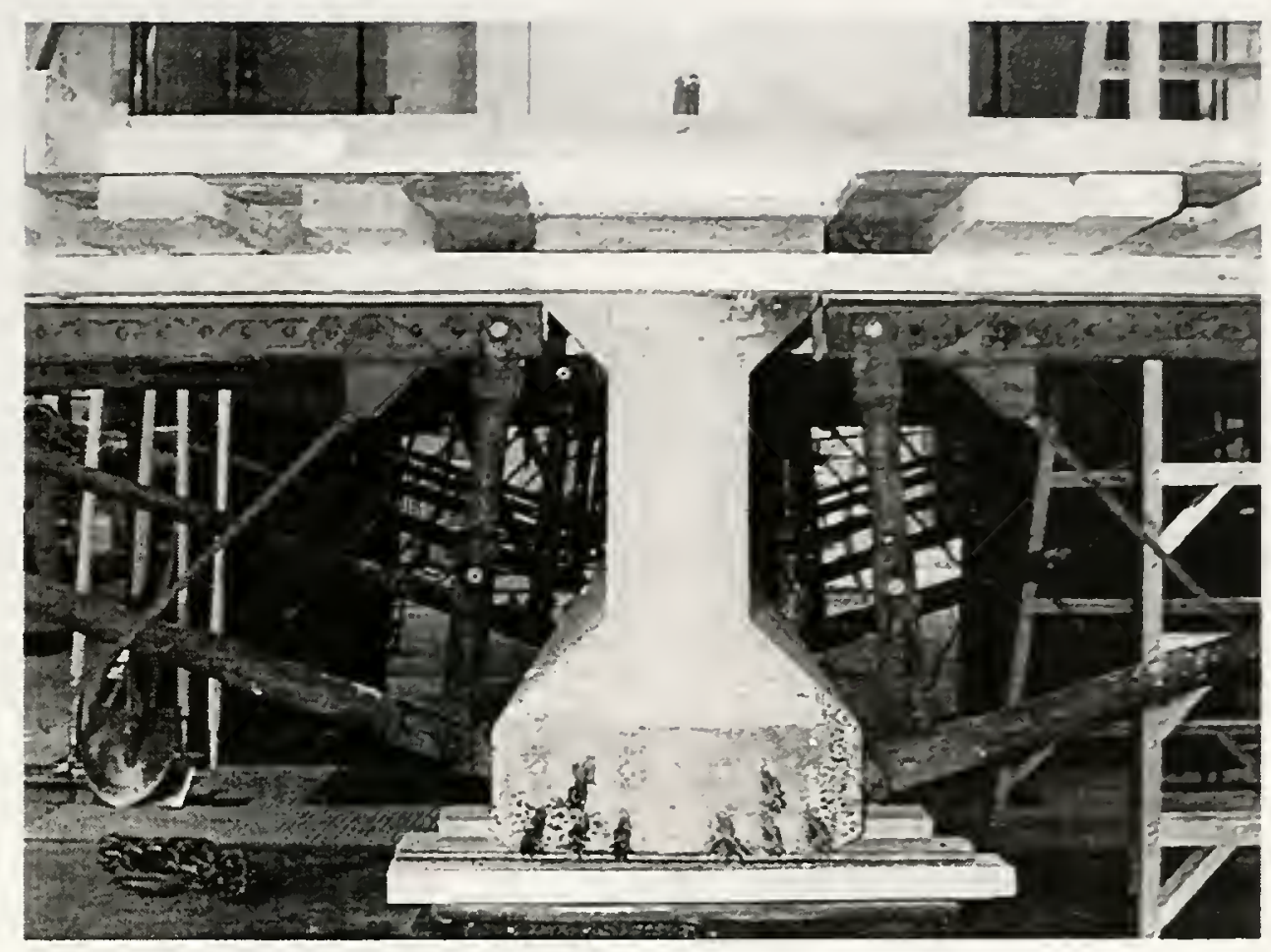

Figure 4.30 Falsework for Cast-In-Place Slab

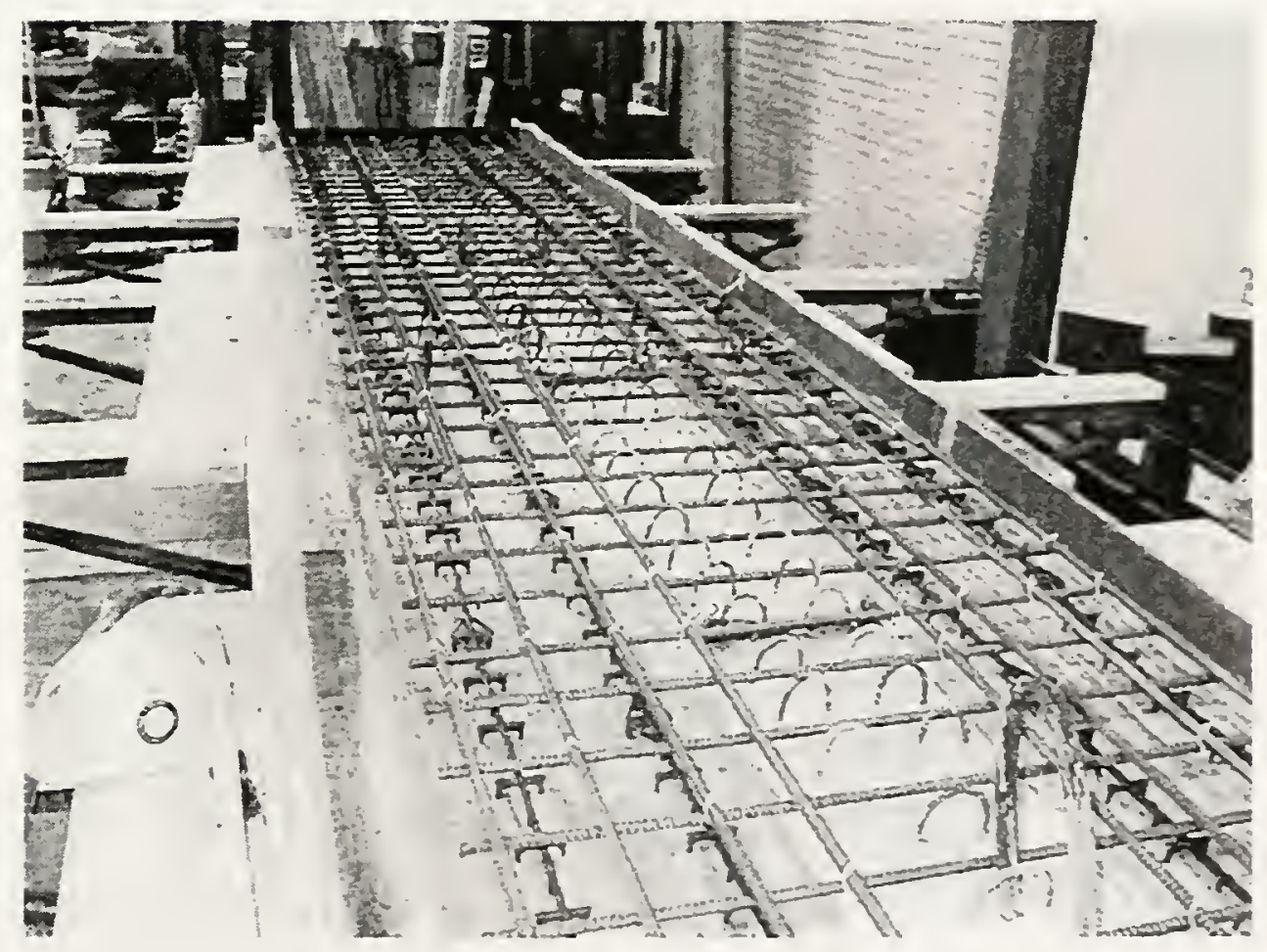

Figure 4.31 Reinforcing Mat for Deck Slab 


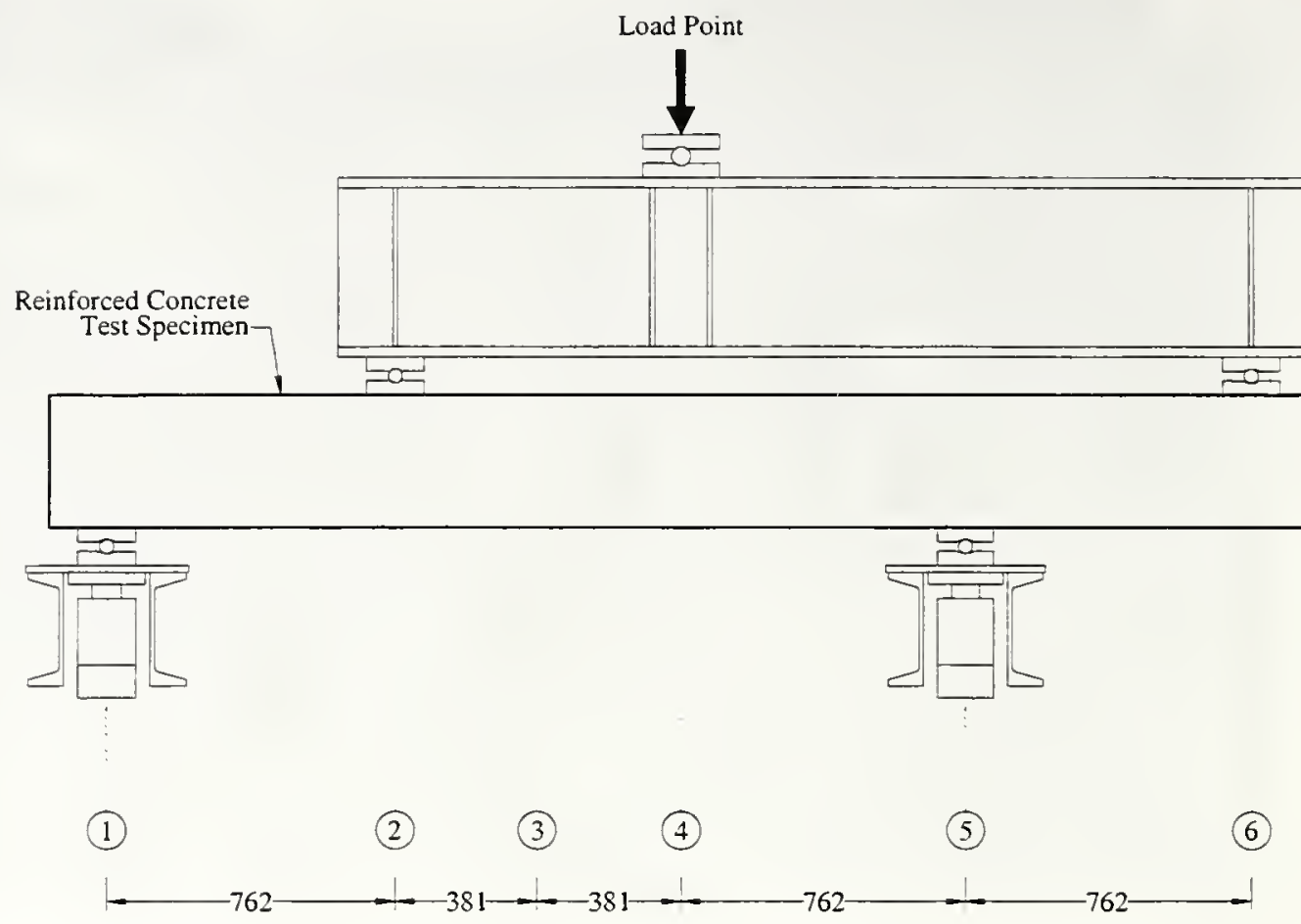

Dimensions shown in millimeters.

Figure 4.32 Location of LVDTs and Dial Gages for Reinforced Concrete Series

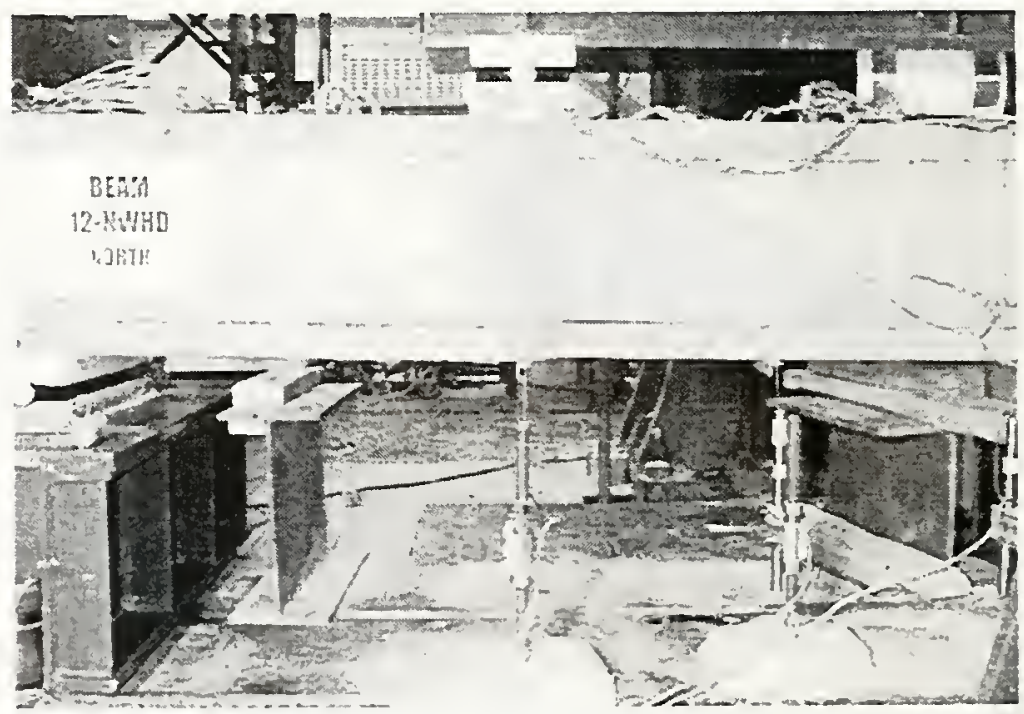

Figure 4.33 LVDTs on West Side of Typical Reinforced Concrete Specimen 


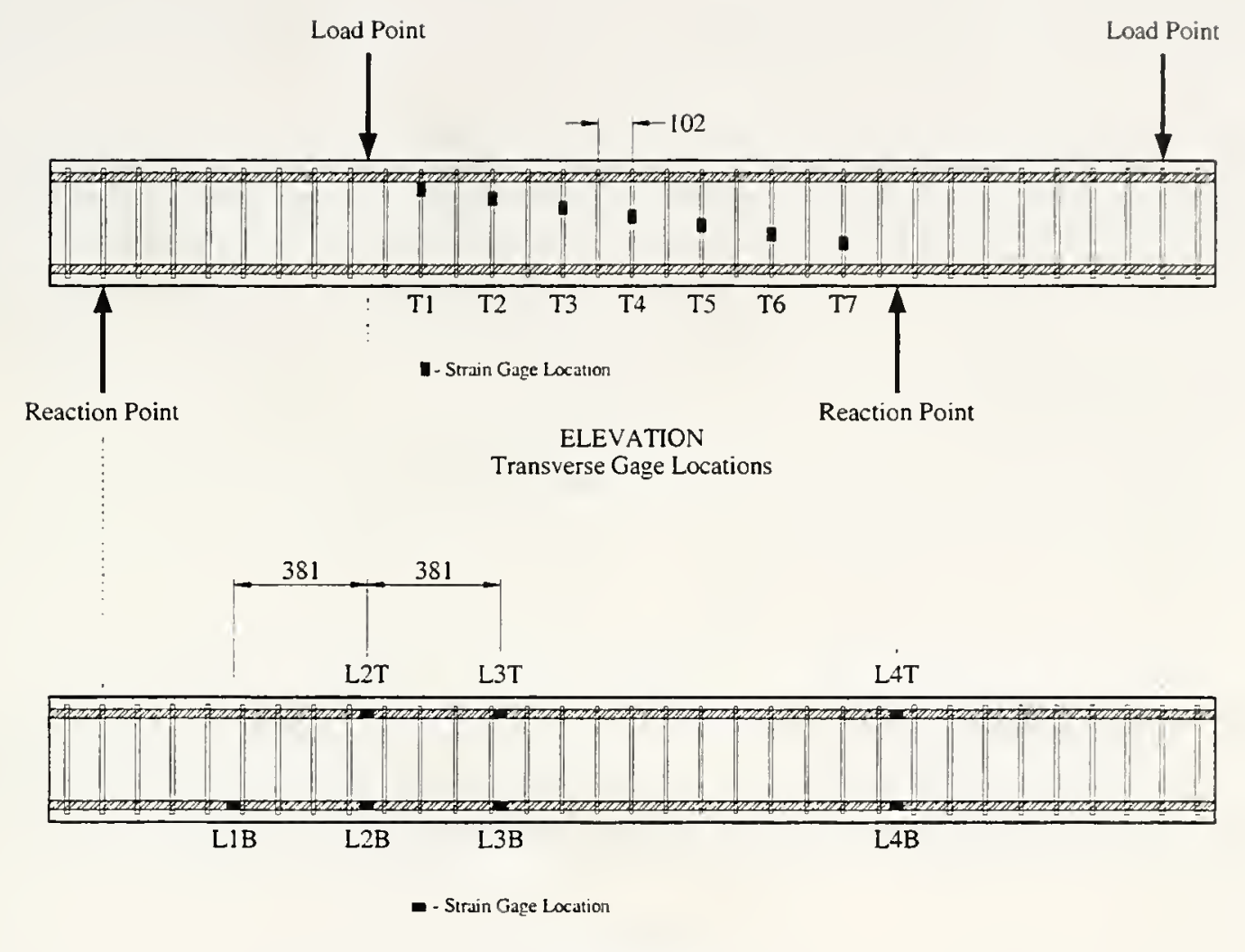

ELEVATION

Longitudinal Gage Locations

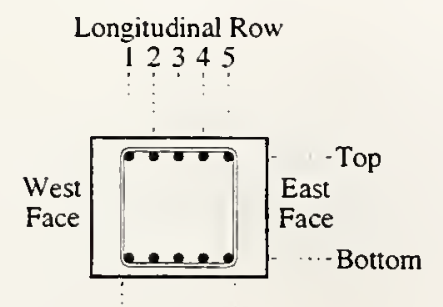

A $\quad$ C
Strain Gage ldentification Scheme:

Strain gage L1B5 refers to:

Longitudinal position $\mathrm{Ll}$

Bottom row of reinforcing bars

Longitudinal row 5

Figure 4.34 Strain Gage Locations for Specimens 1-NWLA and 5-LWLA 


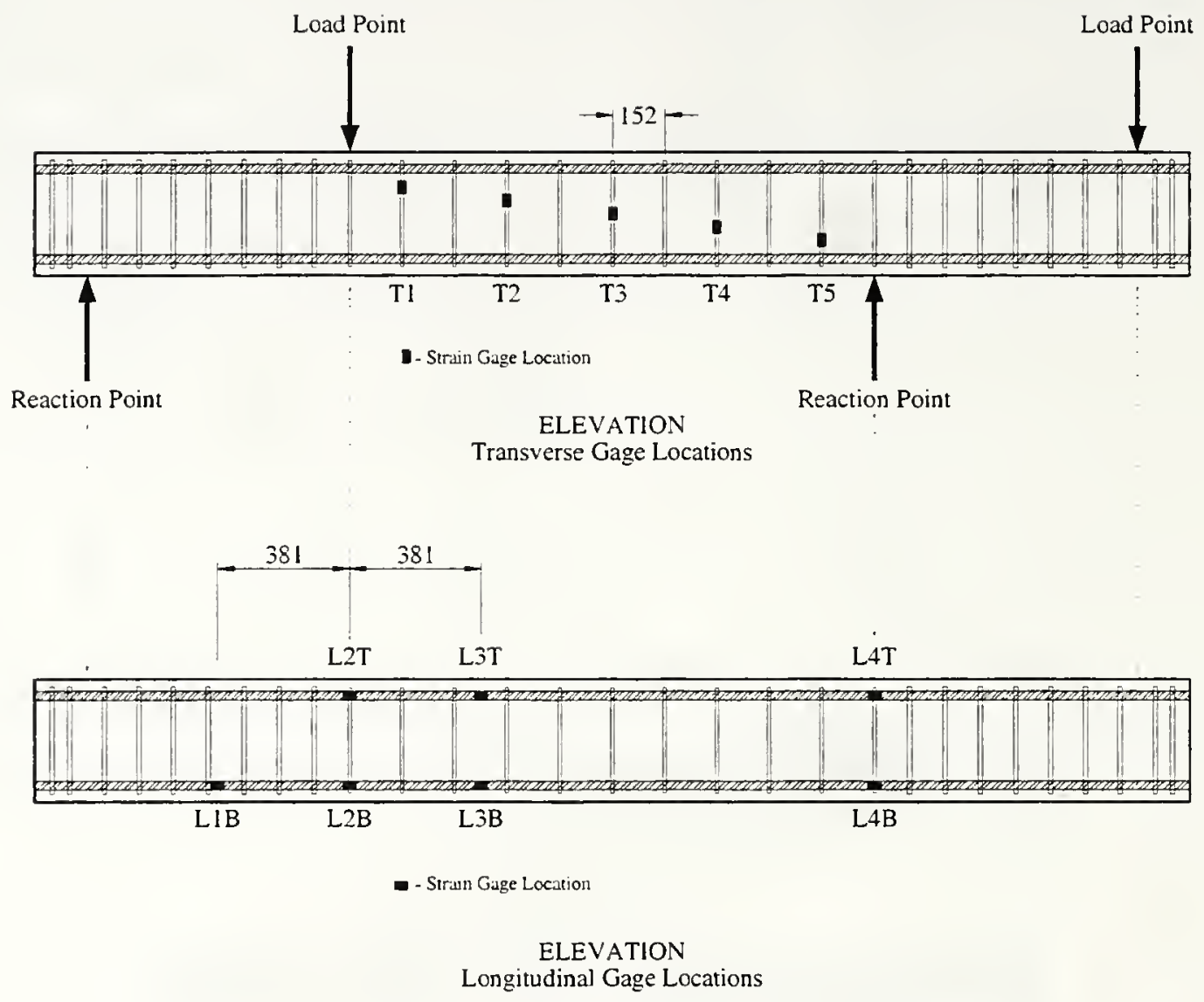

Longitudinal Row

12345

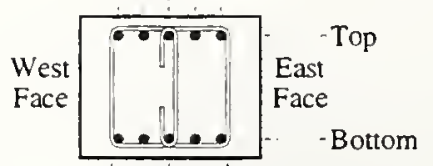

A B C

Stimup Row
Strain Gage Identification Scheme:

Strain gage L1B5 refers to:

Longitudinal position $\mathrm{L}]$

Bottom row of reinforcing bars

Longitudinal row 5

Figure 4.35 Strain Gage Locations for Specimen 2-NWLB and 6-LWLB 


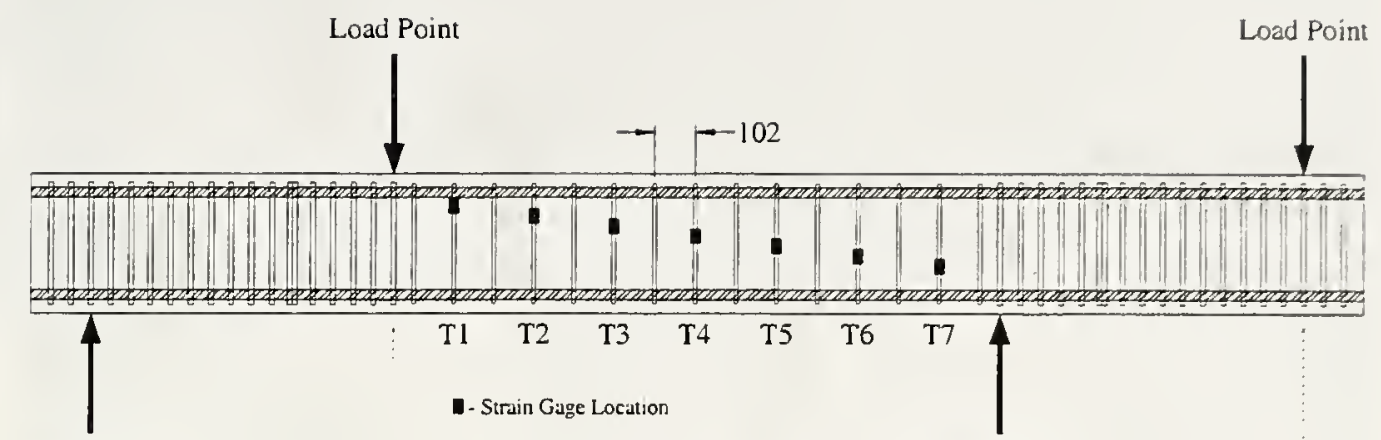

Reaction Point

ELEVATION

Reaction Point

Transverse Gage Locations

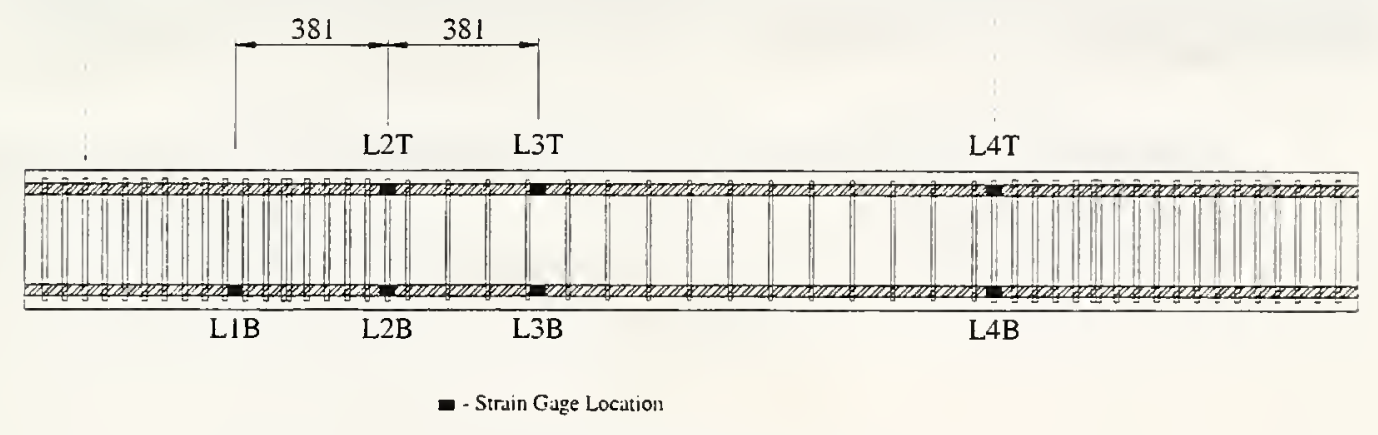

ELEVATION

Longitudinal Gage Locations

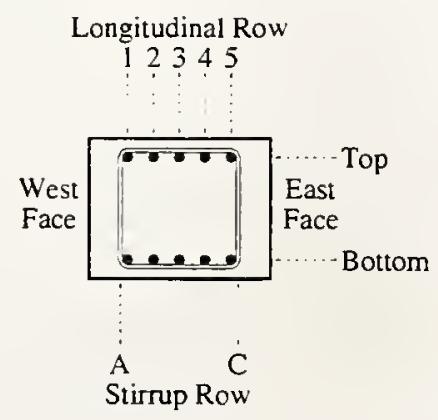

Strain Gage Identification Scheme:

Strain gage L1B5 refers to:

Longitudinal position Ll

Bottom row of reinforcing bars

Longitudinal row 5

Stirrup Row

Dimensions shown in millimeters.

Figure 4.36 Strain Gage Locations for Specimen 3-NWLC and 7-LWLC 


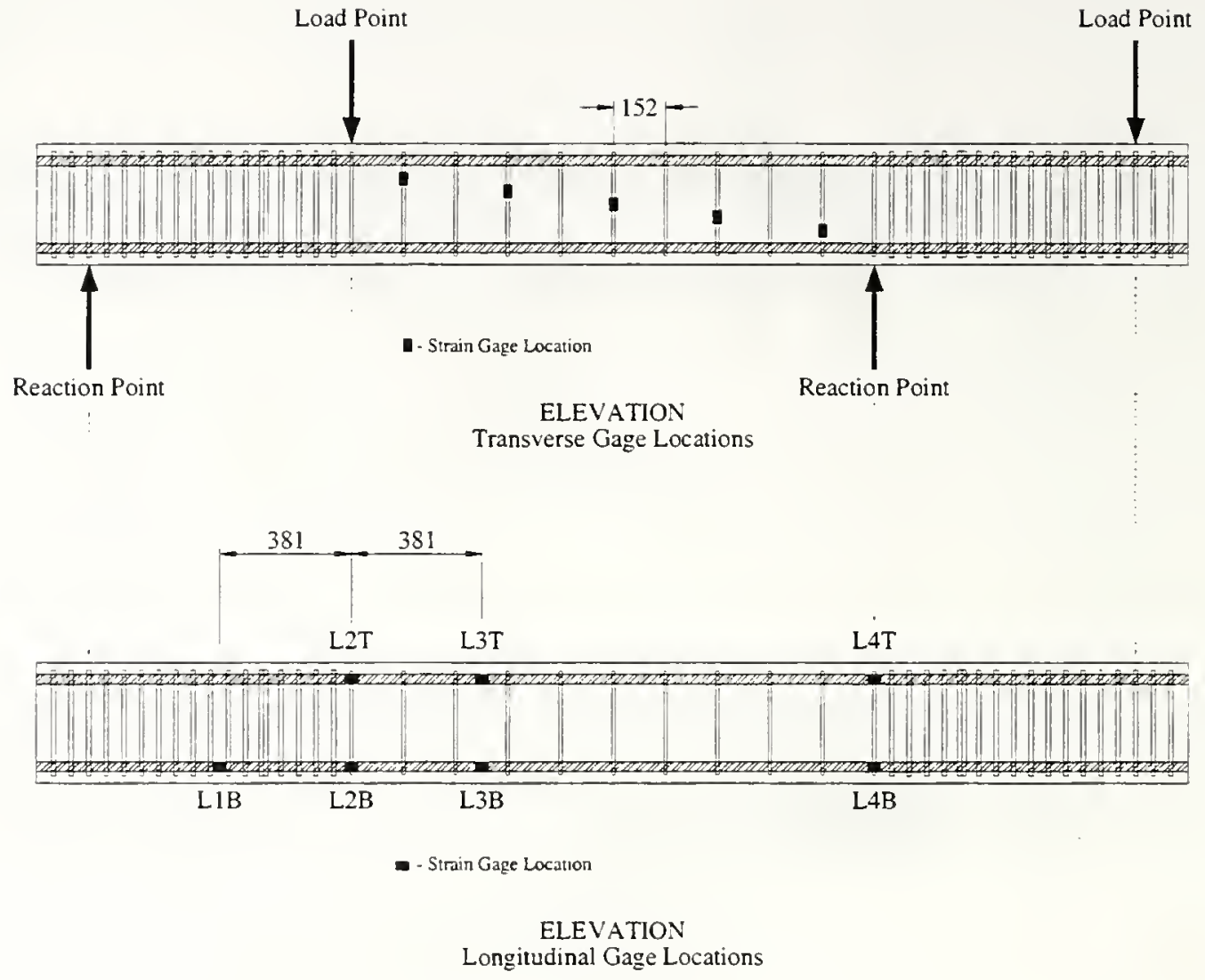

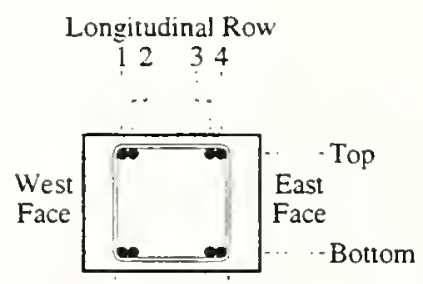

A $\quad$ C Stirrup Row
Strain Gage Identification Scheme:

Strain gage L1B4 refers to:

Longitudinal position Ll

Bottom row of reinforcing bars

Longitudinal row 4

Dimensions shown in millimeters.

Figure 4.37 Strain Gage Locations for Specimen 4-NWLD, 8-LWLD, 9-NWLD, 10-NWHD, 11-LWHD, and 12-NWHD 


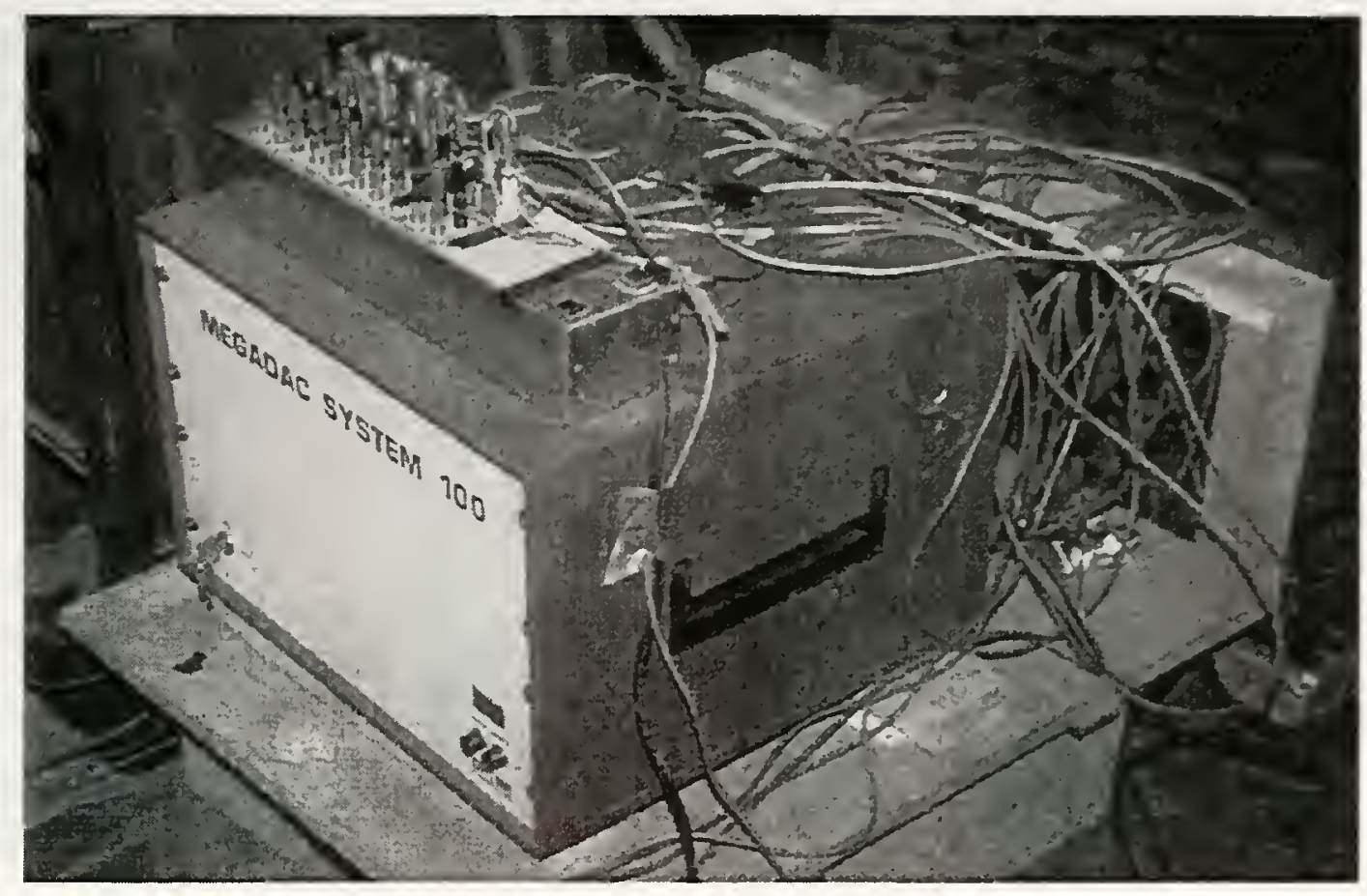

Figure 4.38 Data Acquisition Unit 


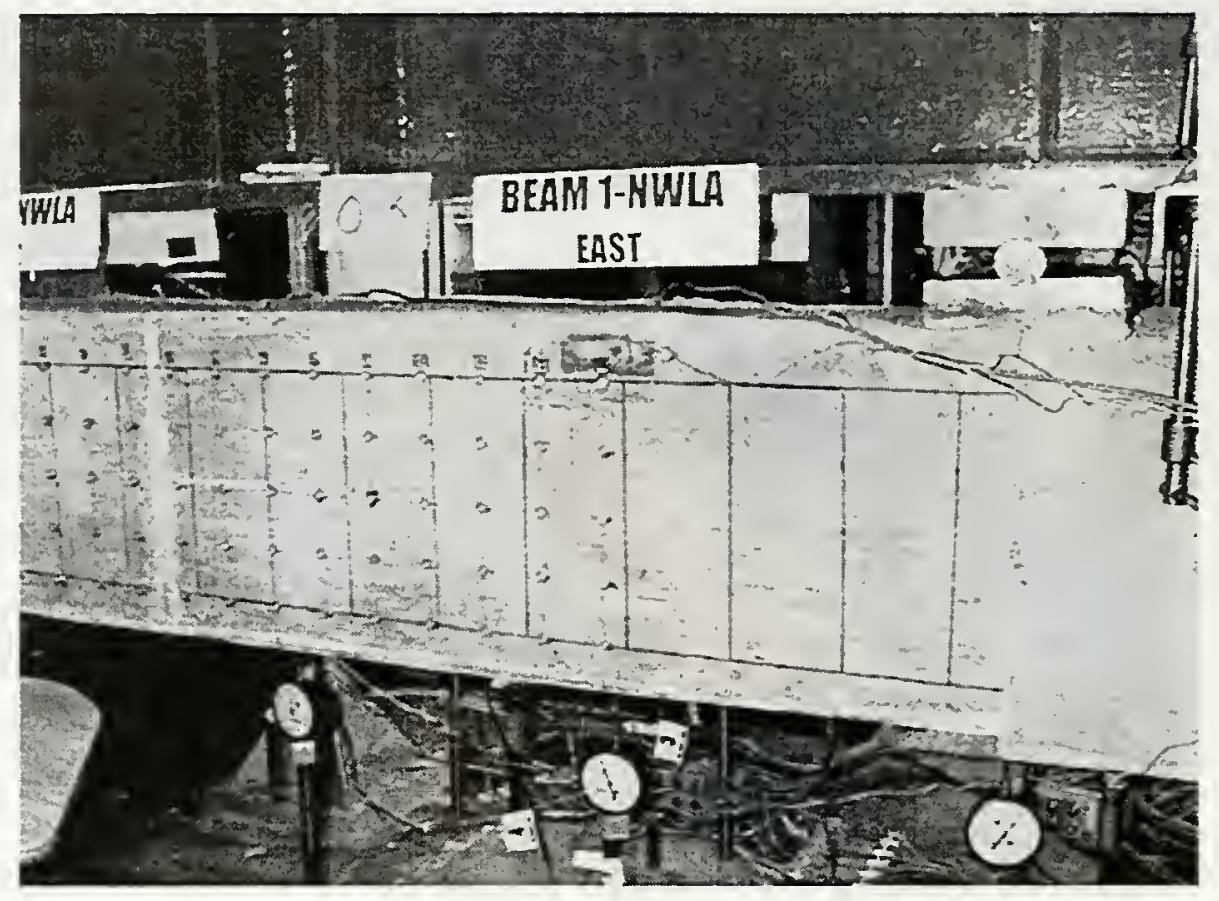

Figure 4.39 Dial Gages Underneath East Side of Reinforced Concrete Specimen

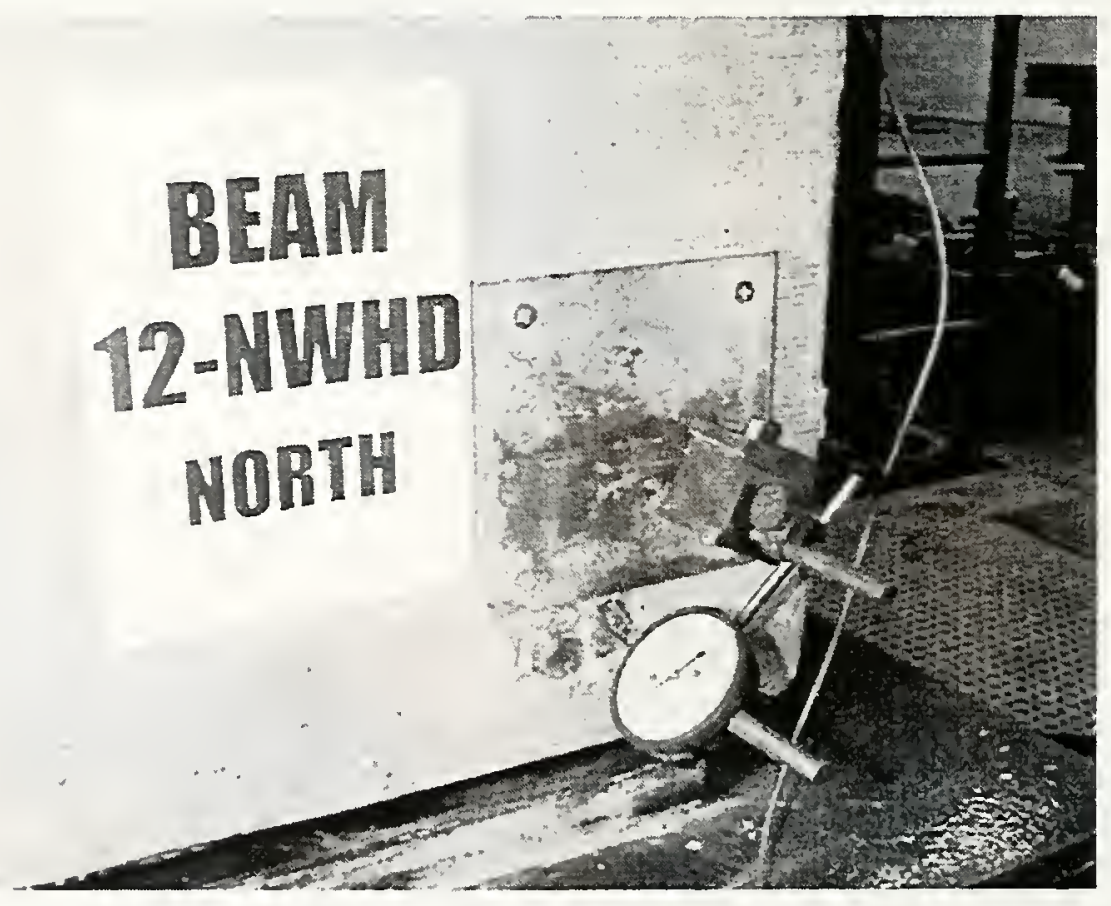

Figure 4.40 Dial Gage for Slip Measurement 


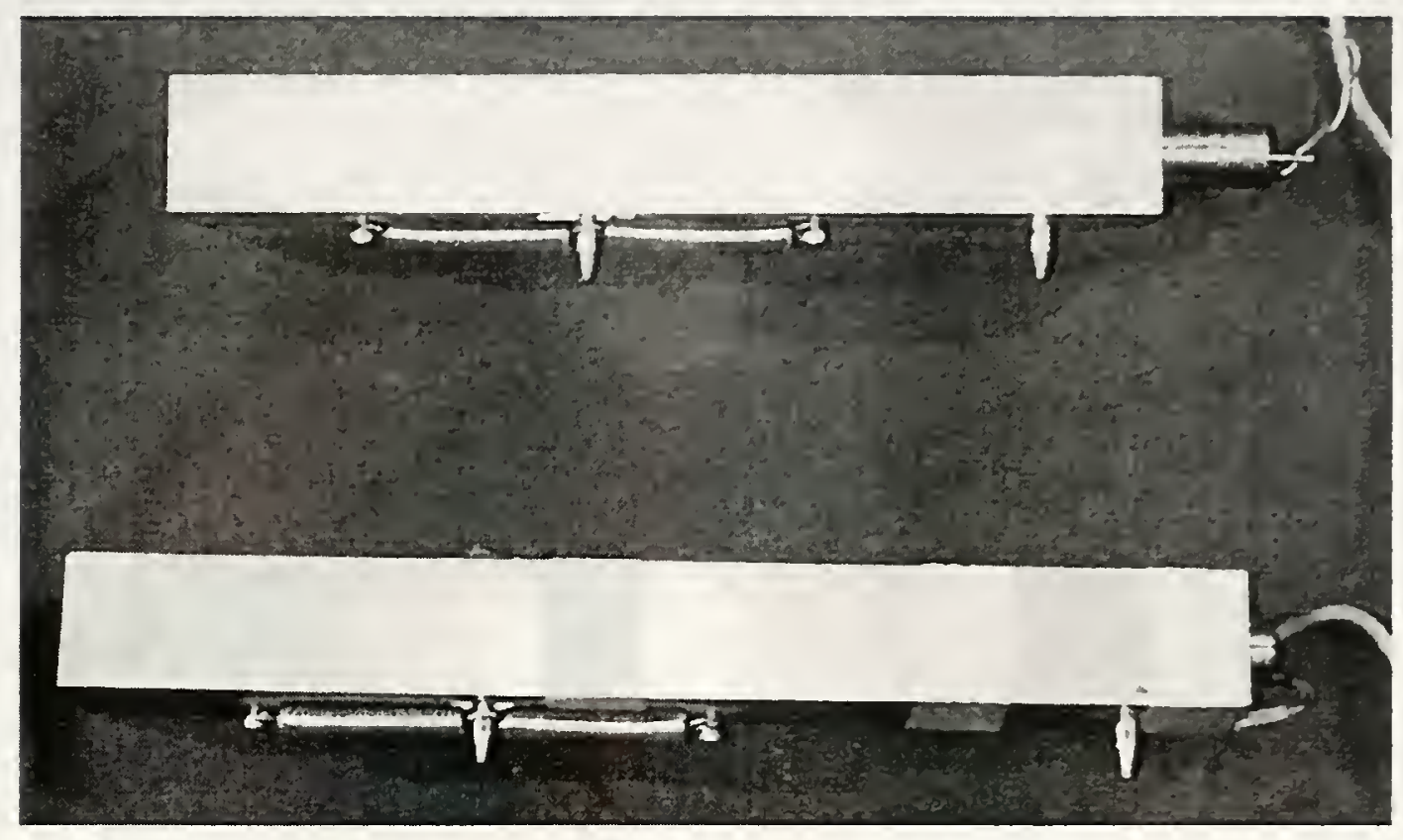

Figure 4.41 Electronic Whittemore Strain Gages

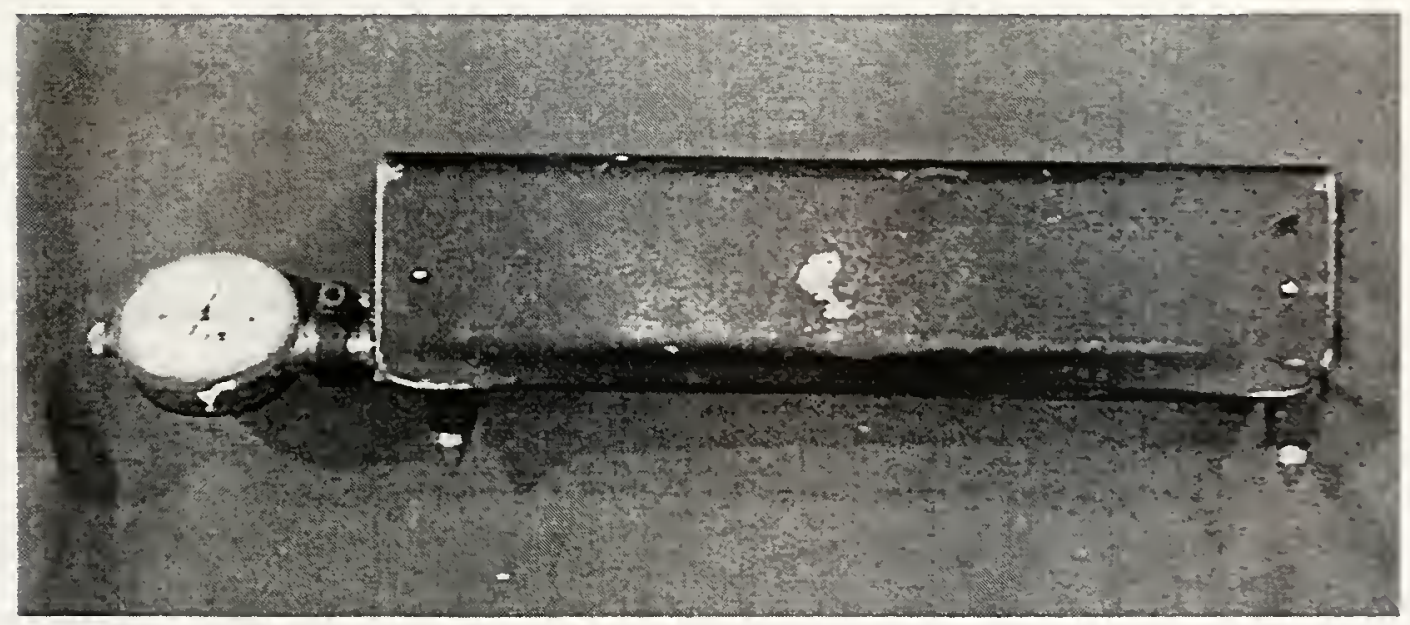

Figure 4.42 Mechanical Whittemore Strain Gage 

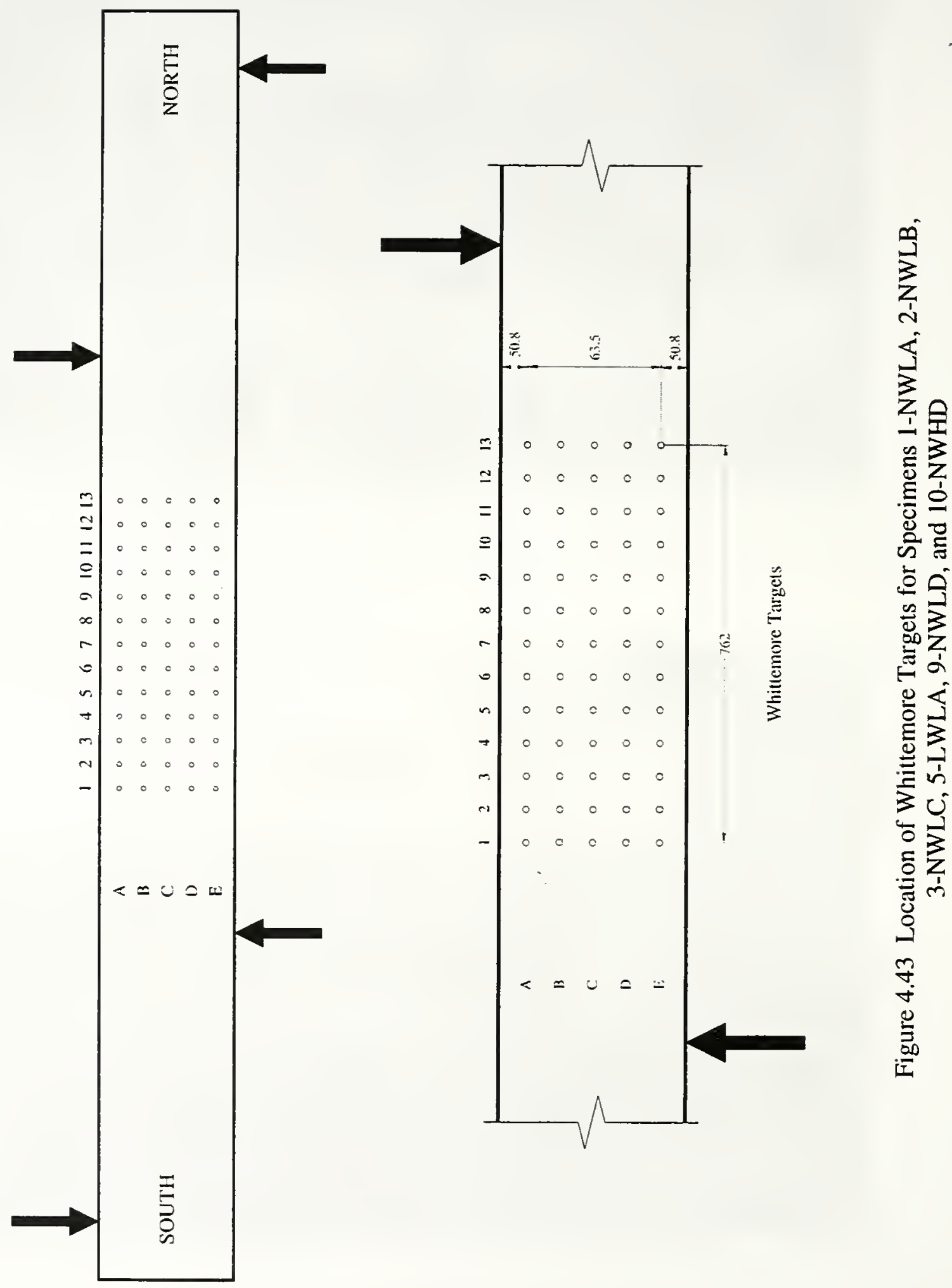


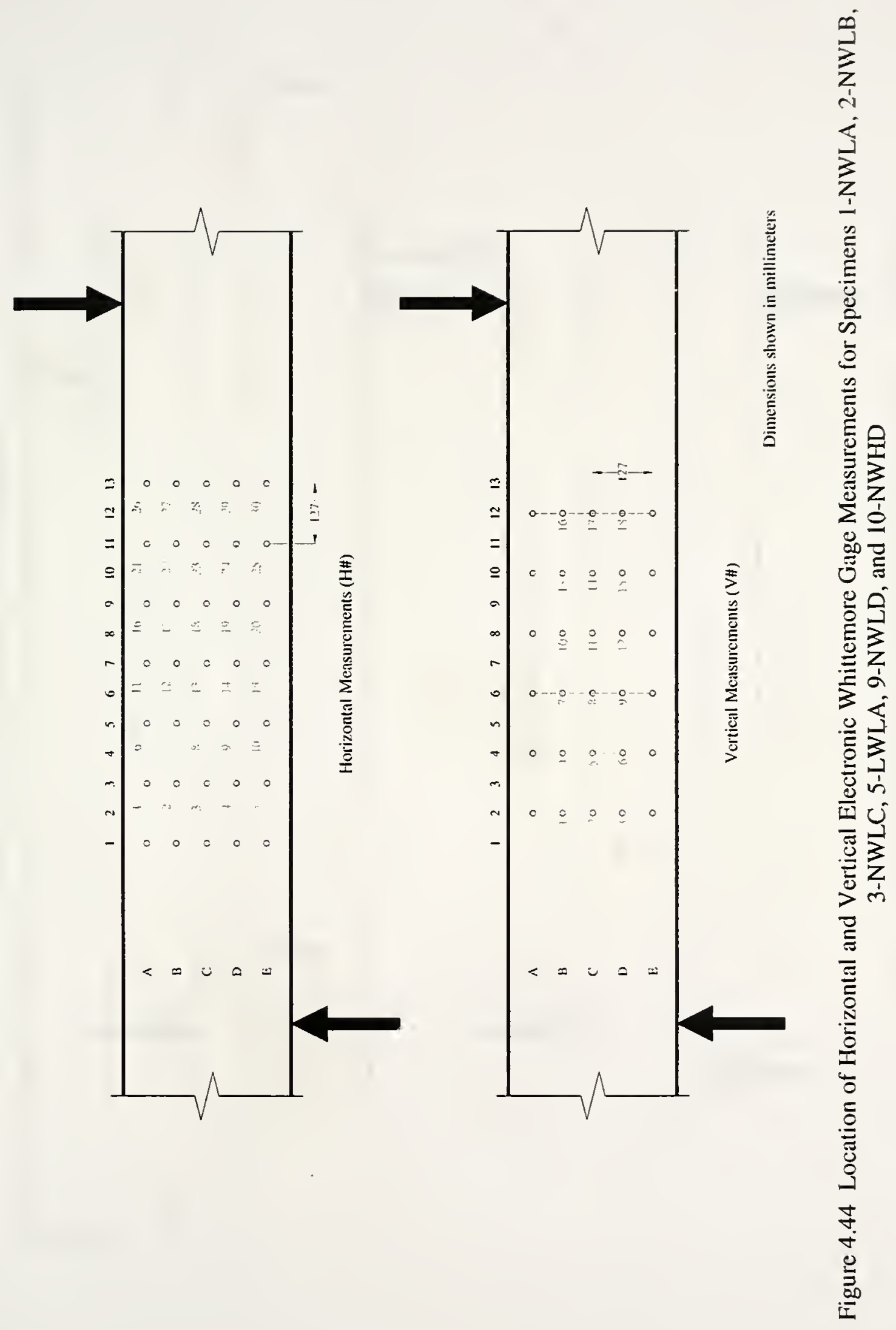



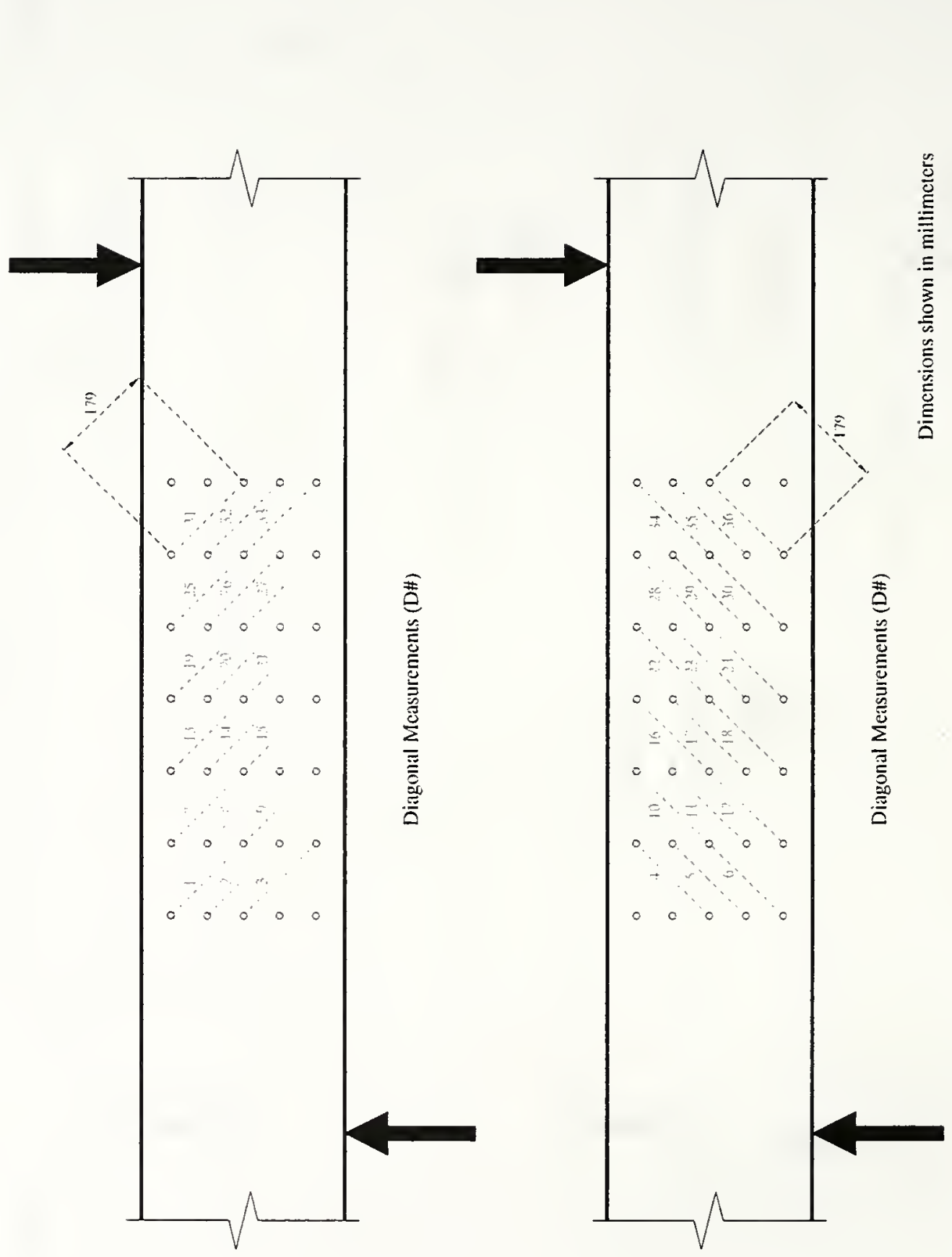

$\sum_{i}^{\infty}$

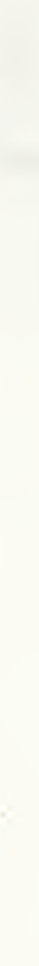

完

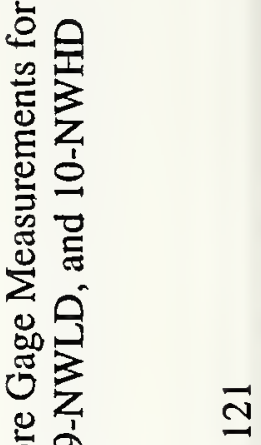

总

告

旁 


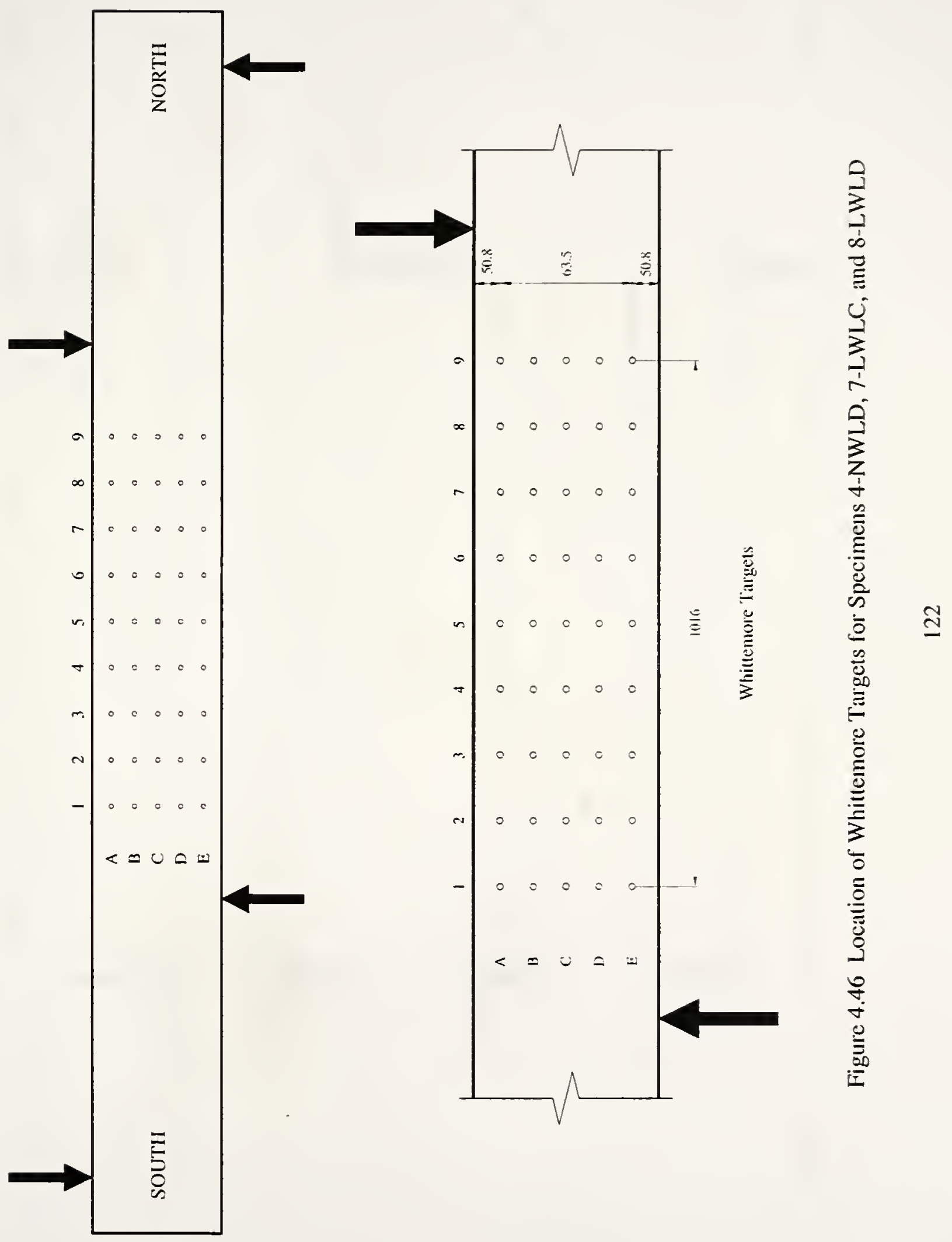




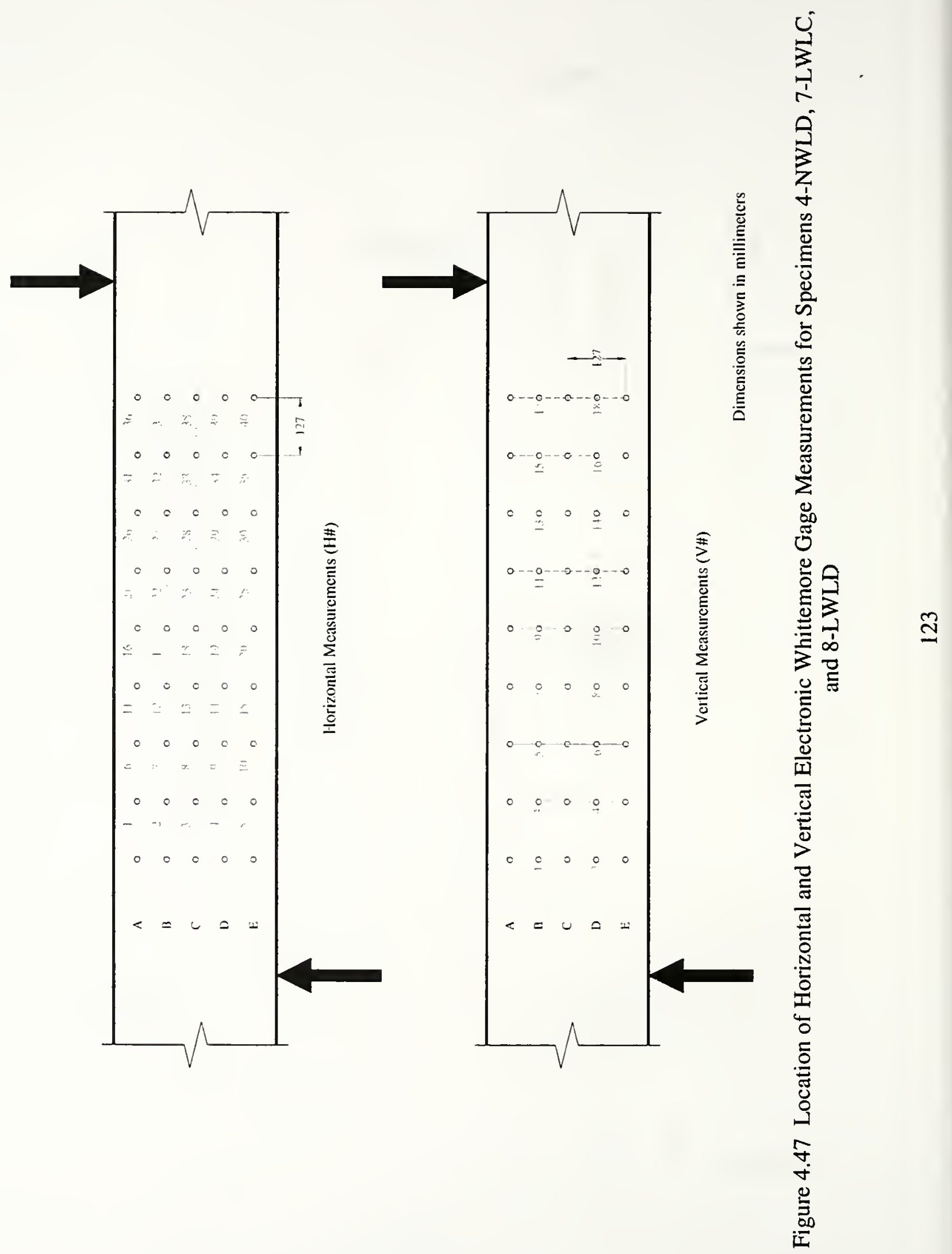




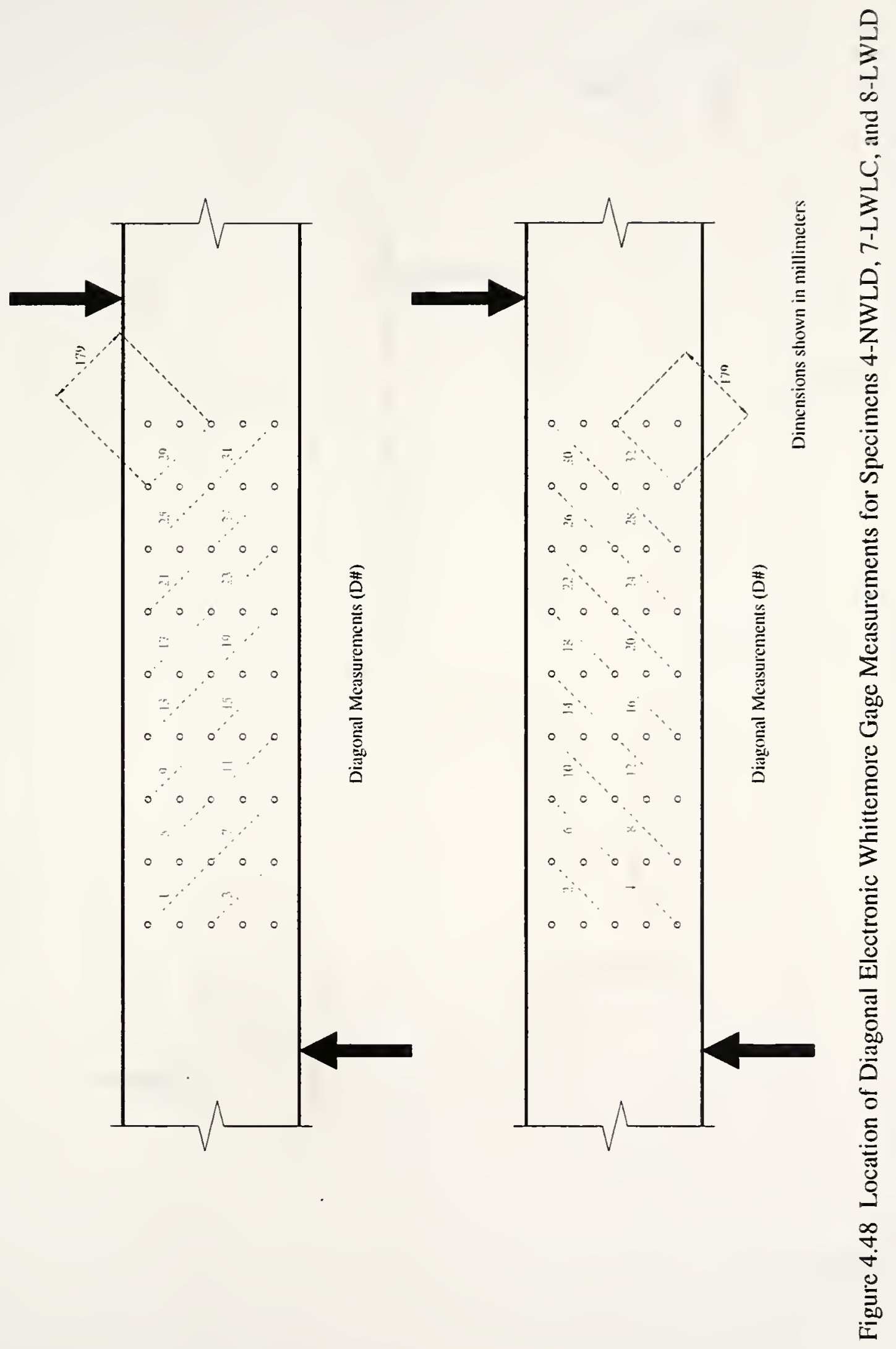



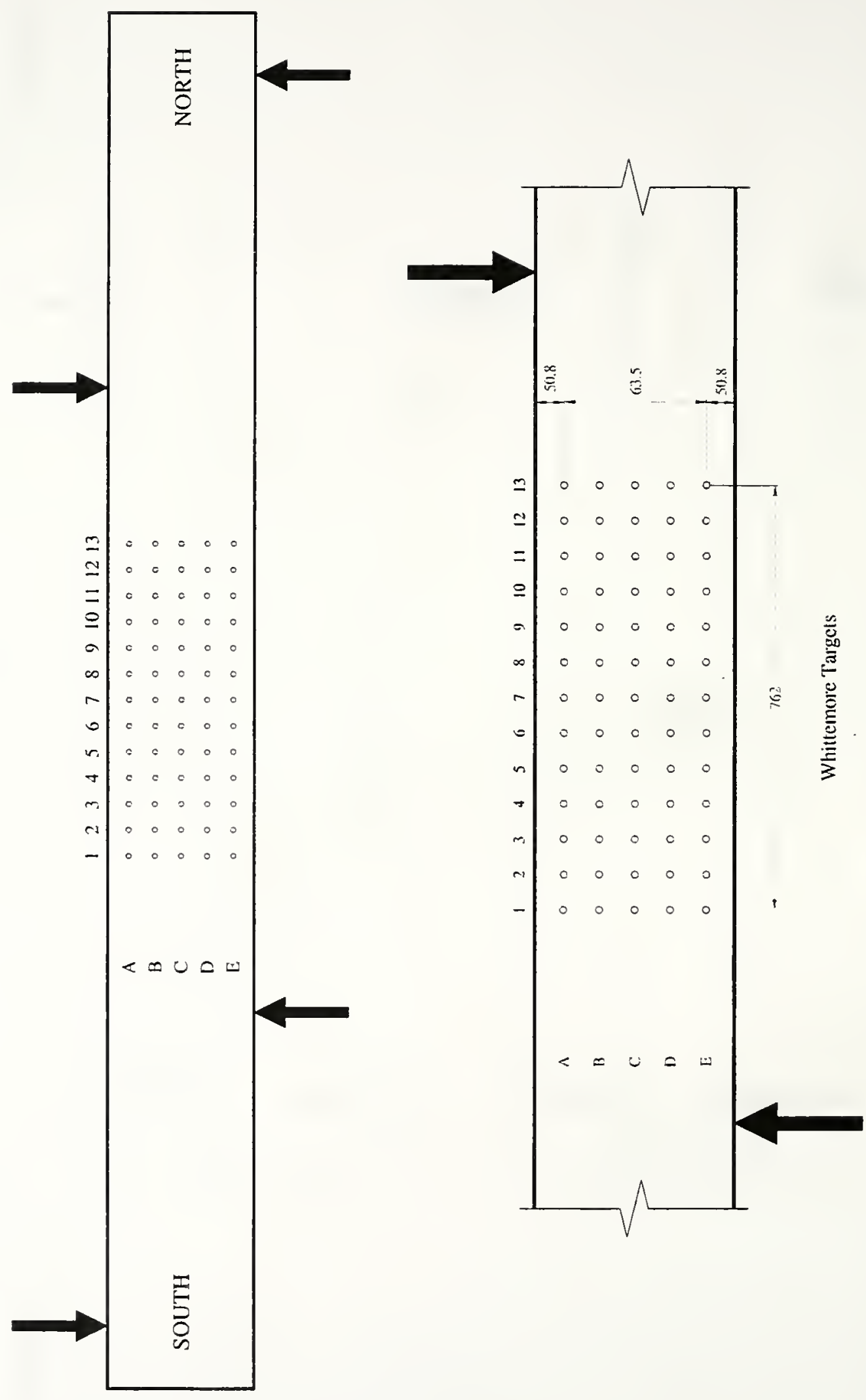

ป 


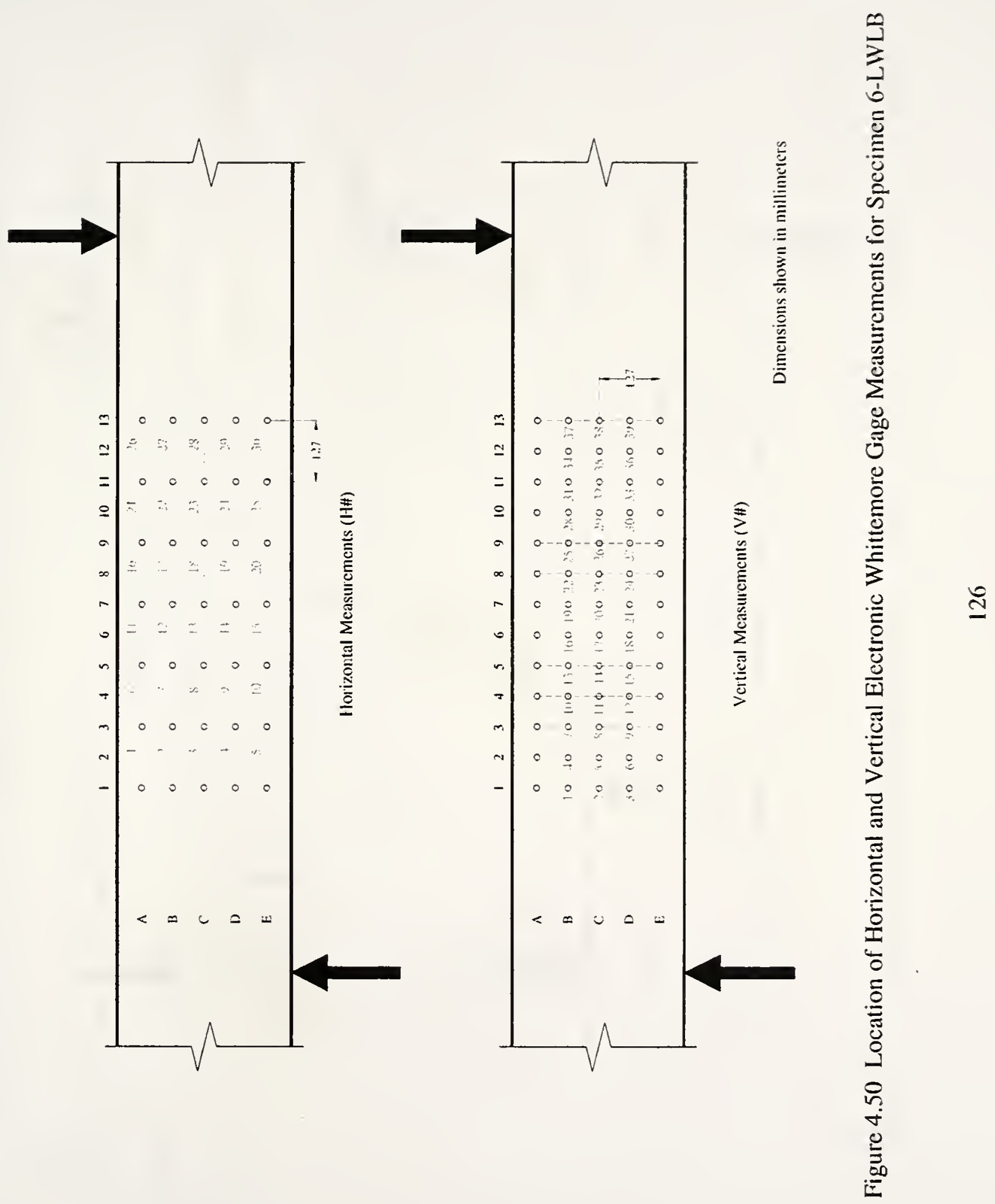



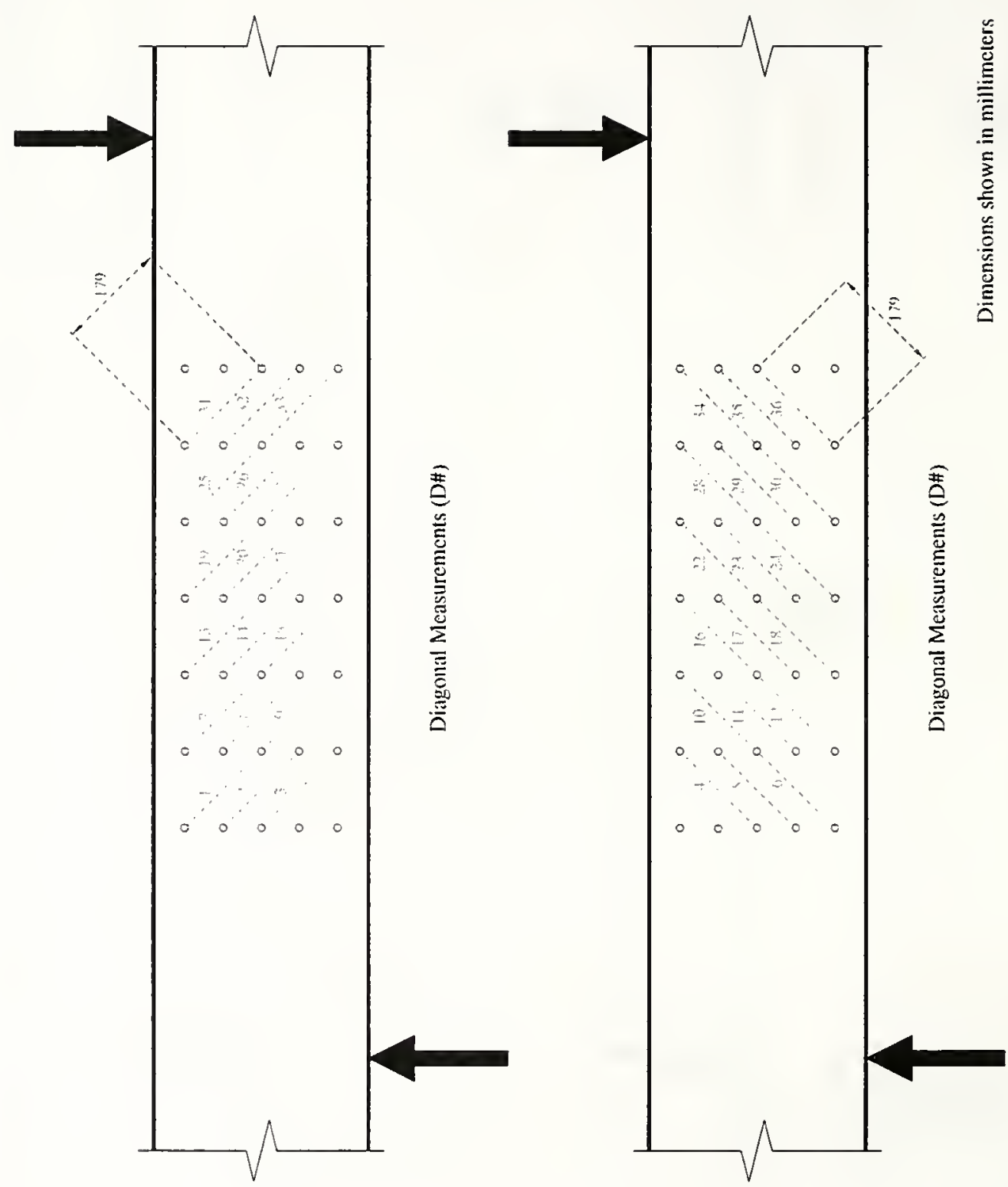

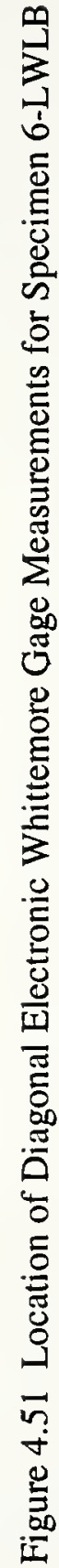



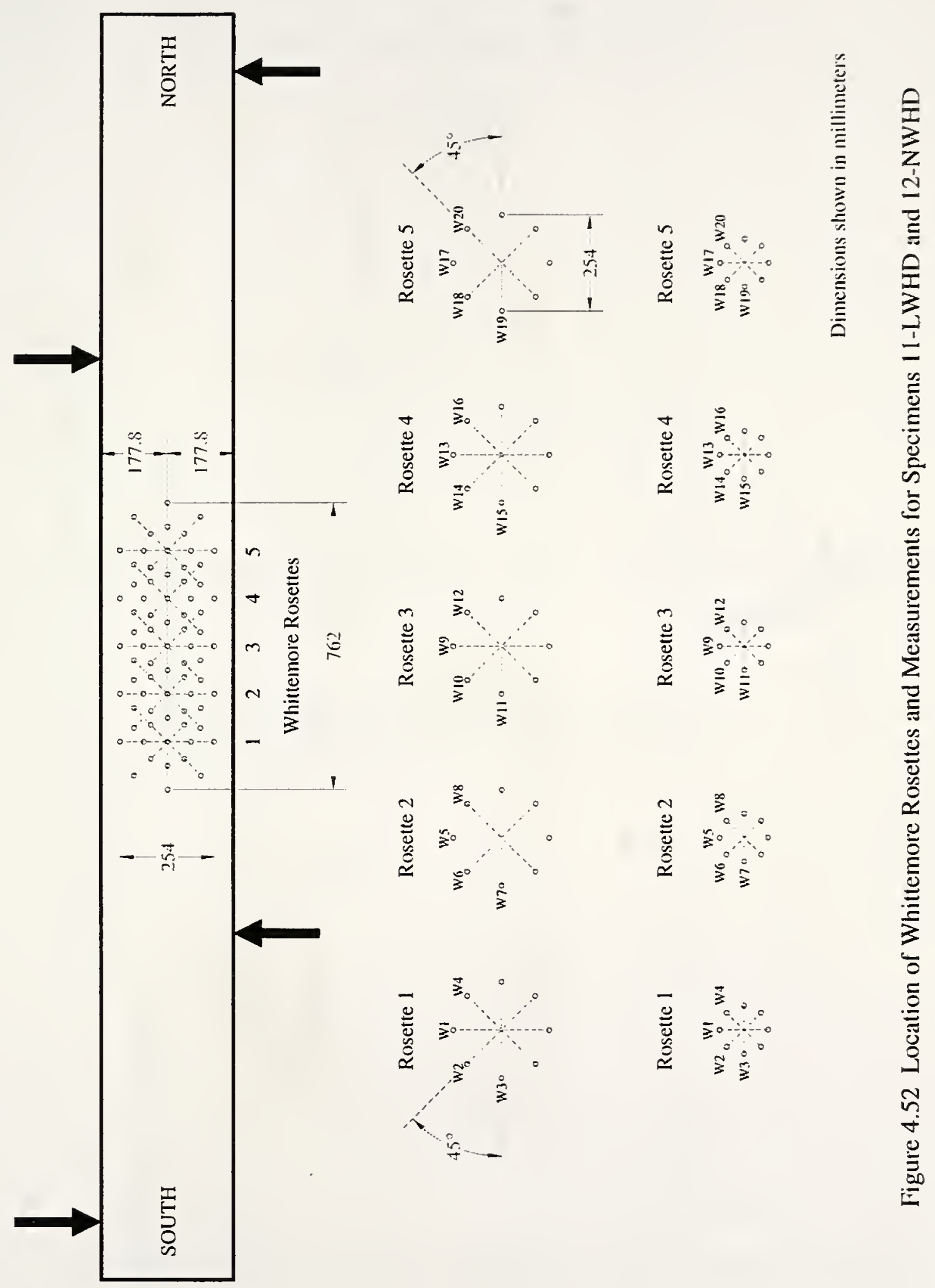

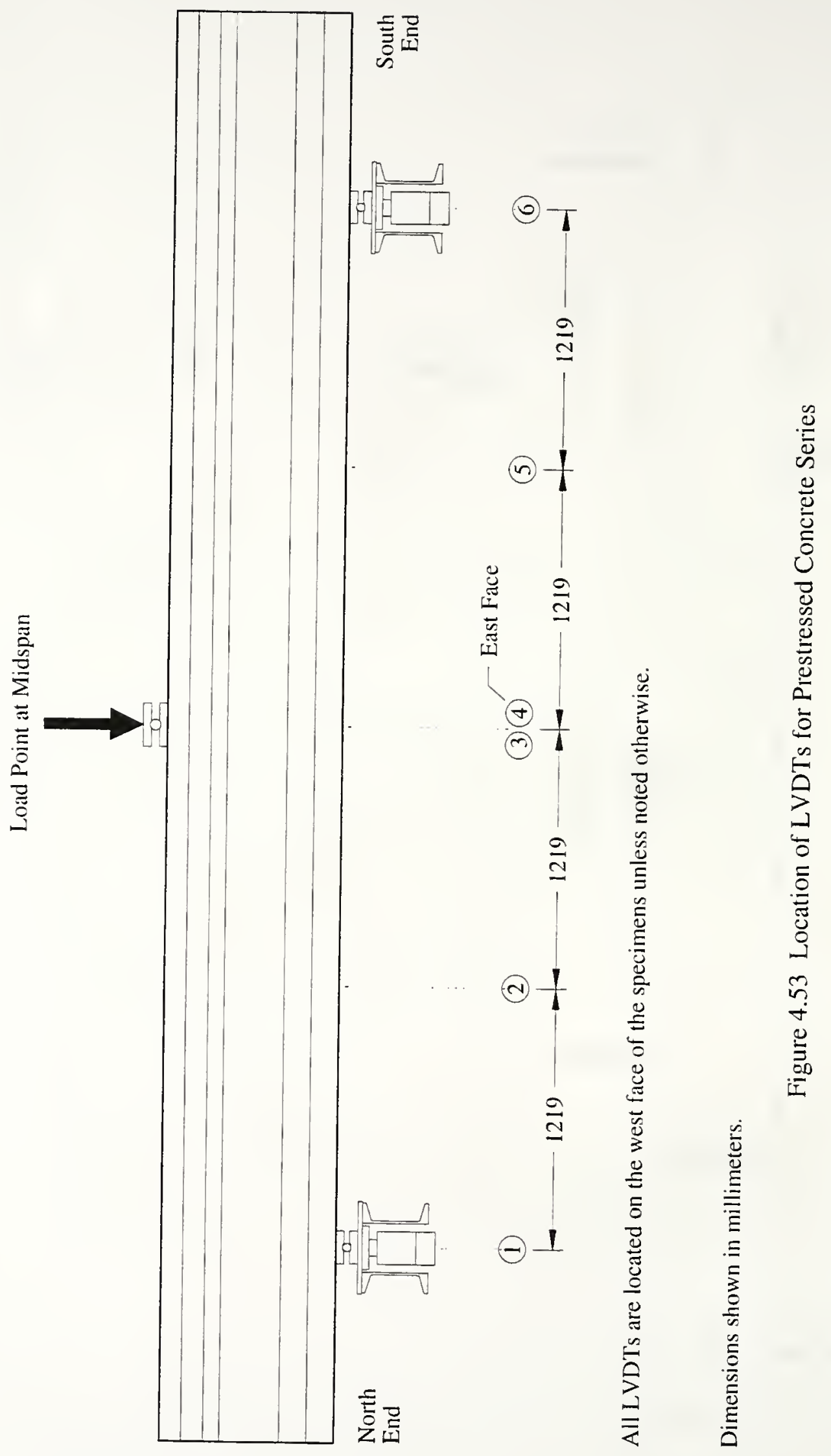


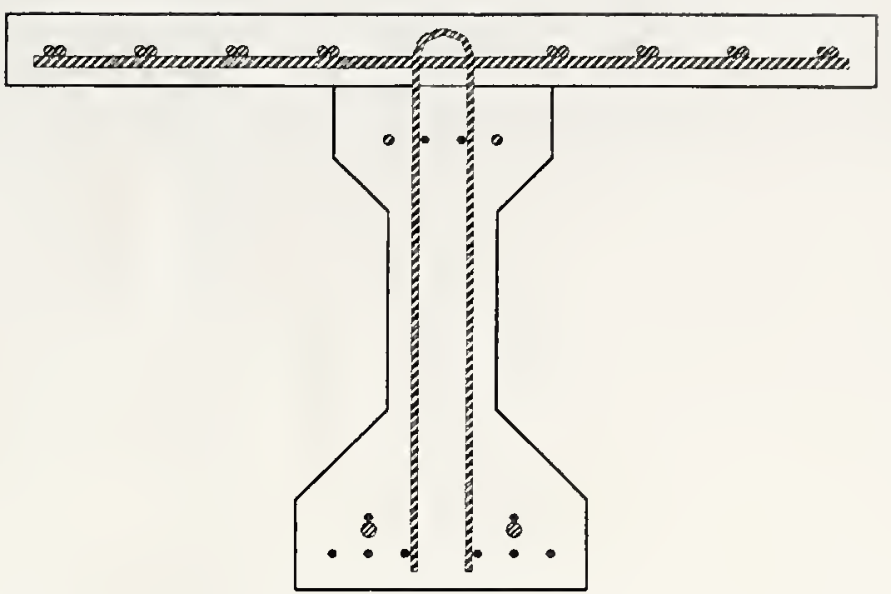
S: $\begin{array}{lllllll}2 & 2 & 4 & 5 & 6 & \text { Bottom Row of Strand }\end{array}$
S: $\quad 7 \quad 8 \quad$ Second Row of Strand
L: 1 Bottom Row of Mild Deformed Bars

Figure 4.54 Location of Longitudinal Strain Gages in Specimen PC6S at Midspan

D1 D2

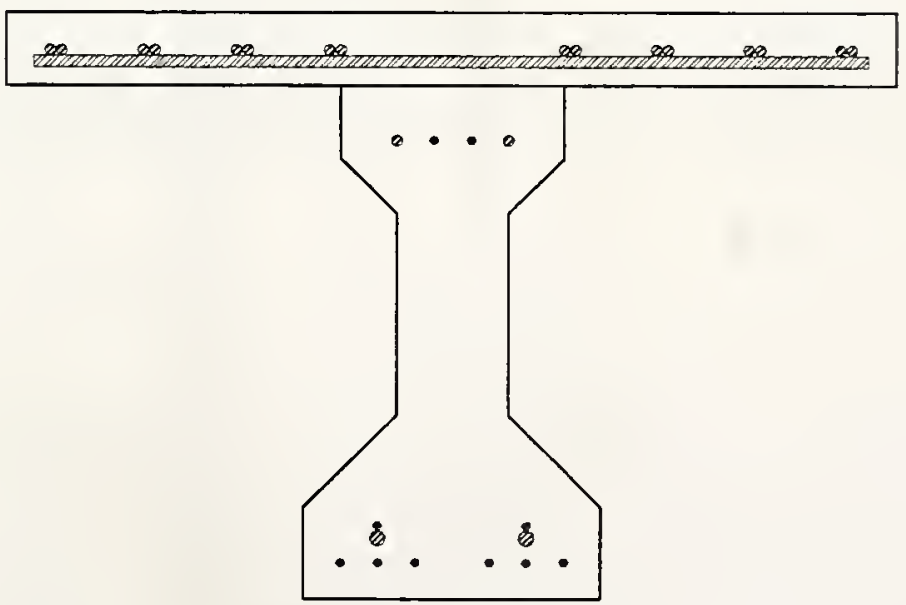
S: $1 \quad 2 \quad 3$
456
Bottom Row of Strand
s: $\quad 7$
$8 \quad$ Second Row of Strand
L: 1
2 Bottom Row of Mild Deformed Bars

Figure 4.55 Location of Longitudinal Strain Gages in Specimen PC6N at Midspan 


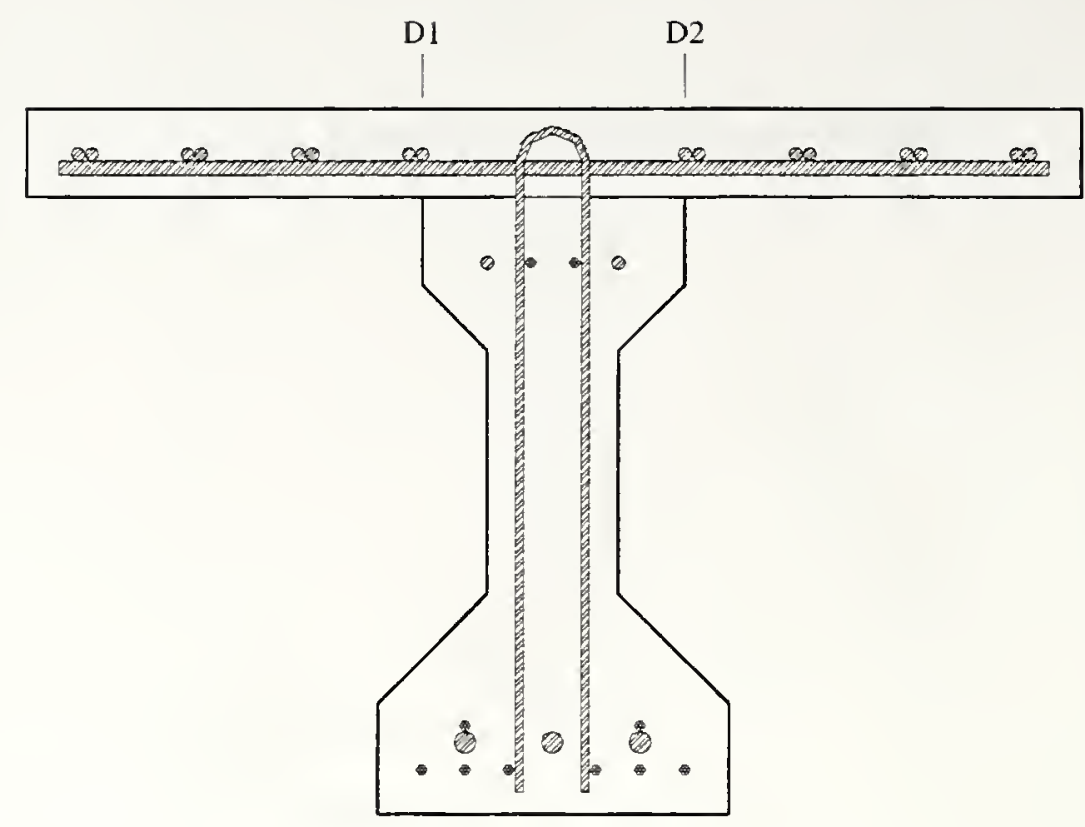

L: $\quad 1 \quad 2 \quad 3 \quad$ Bottom Row of Mild Deformed Bars

Figure 4.56 Location of Longitudinal Strain Gages in Specimen PC10S at Midspan

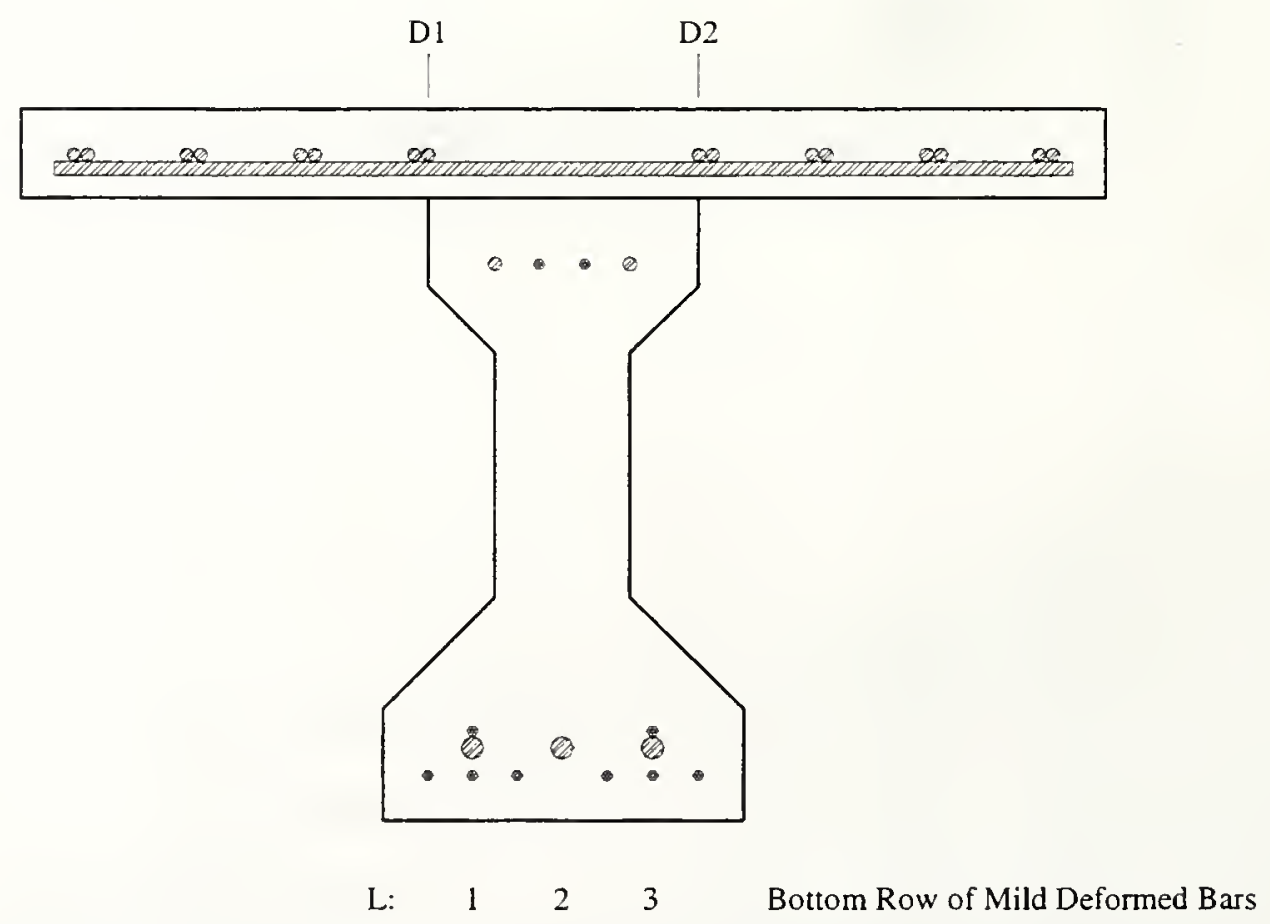

Figure 4.57 Location of Longitudinal Strain Gages in Specimen PC10N at Midspan 


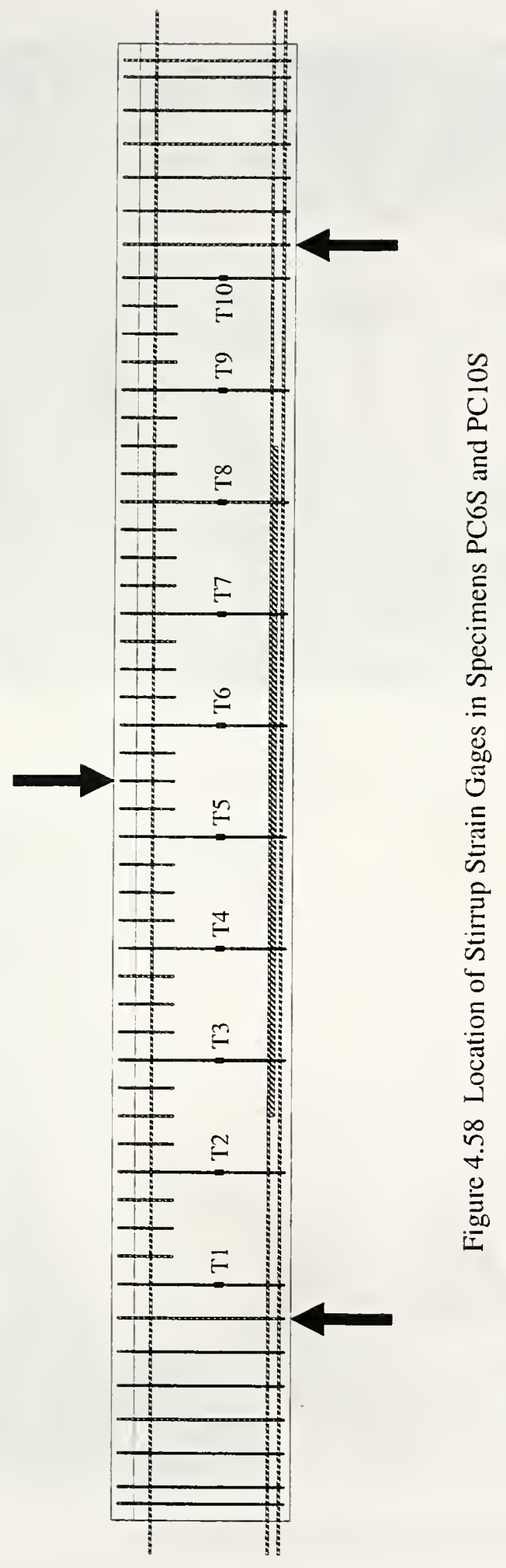




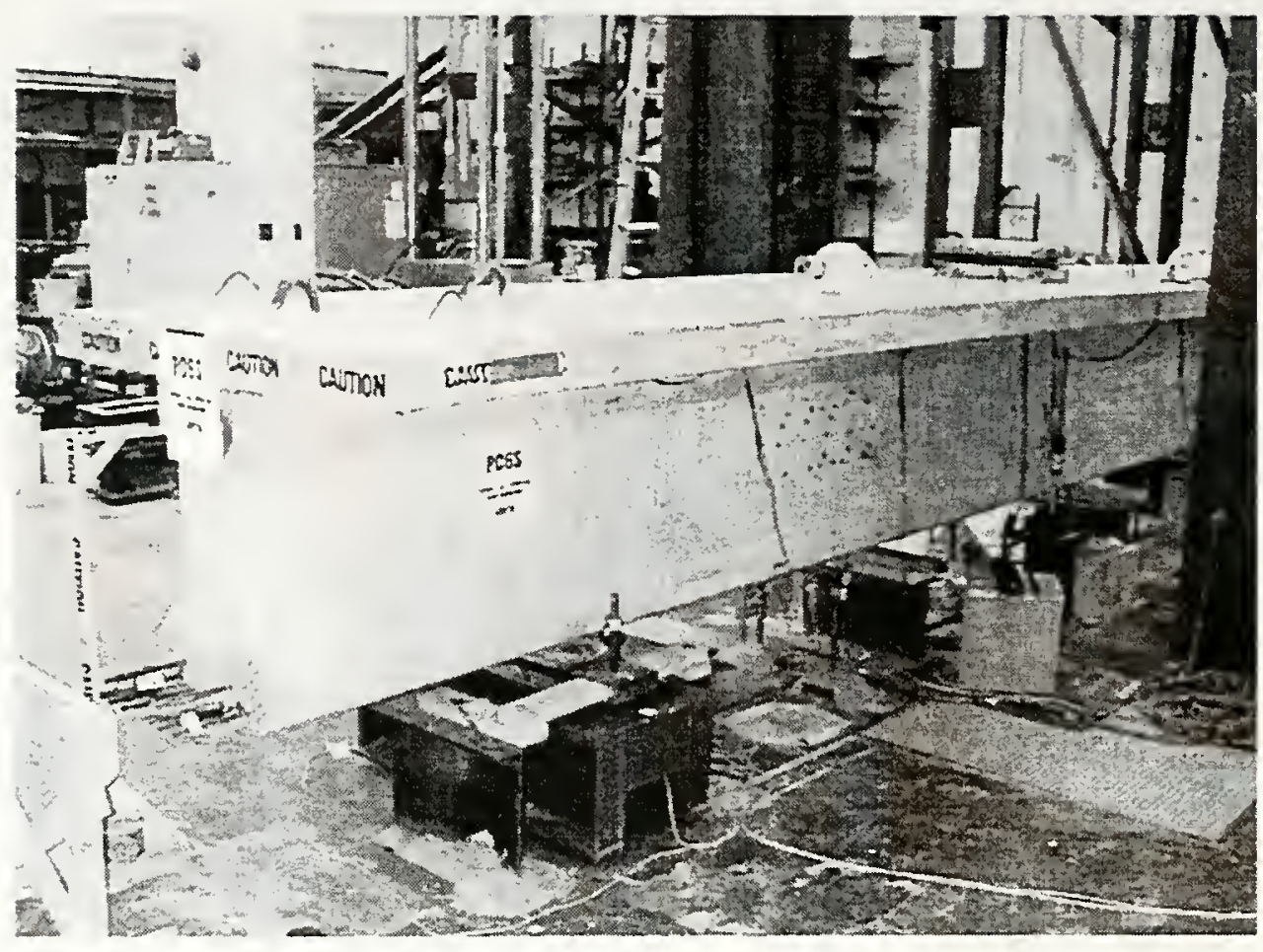

Figure 4.59 East Side of Prestressed Concrete Setup (PC6S) Showing Location of Dial Gages

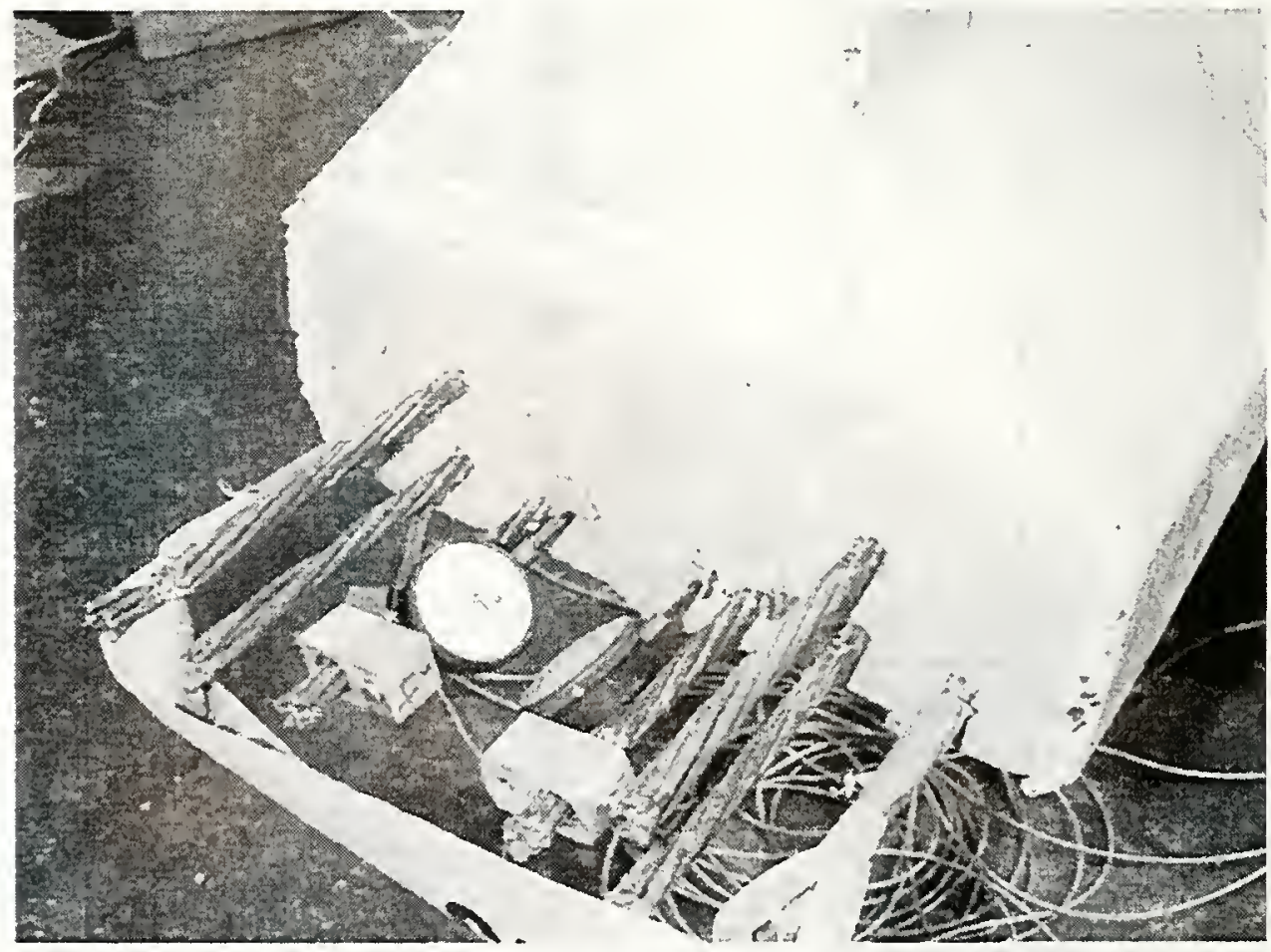

Figure 4.60 Dial Gage for Strand Slip Measurement 

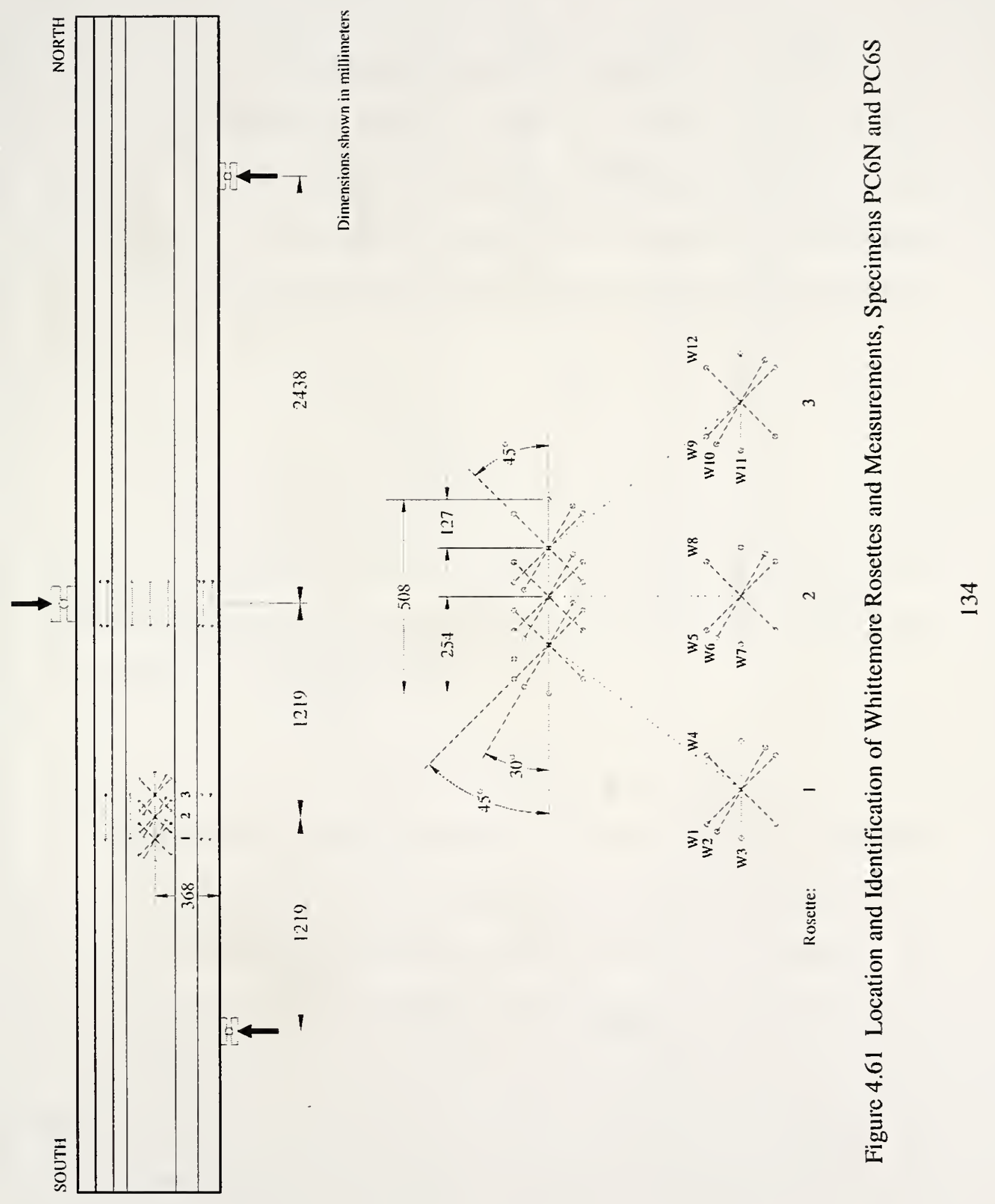

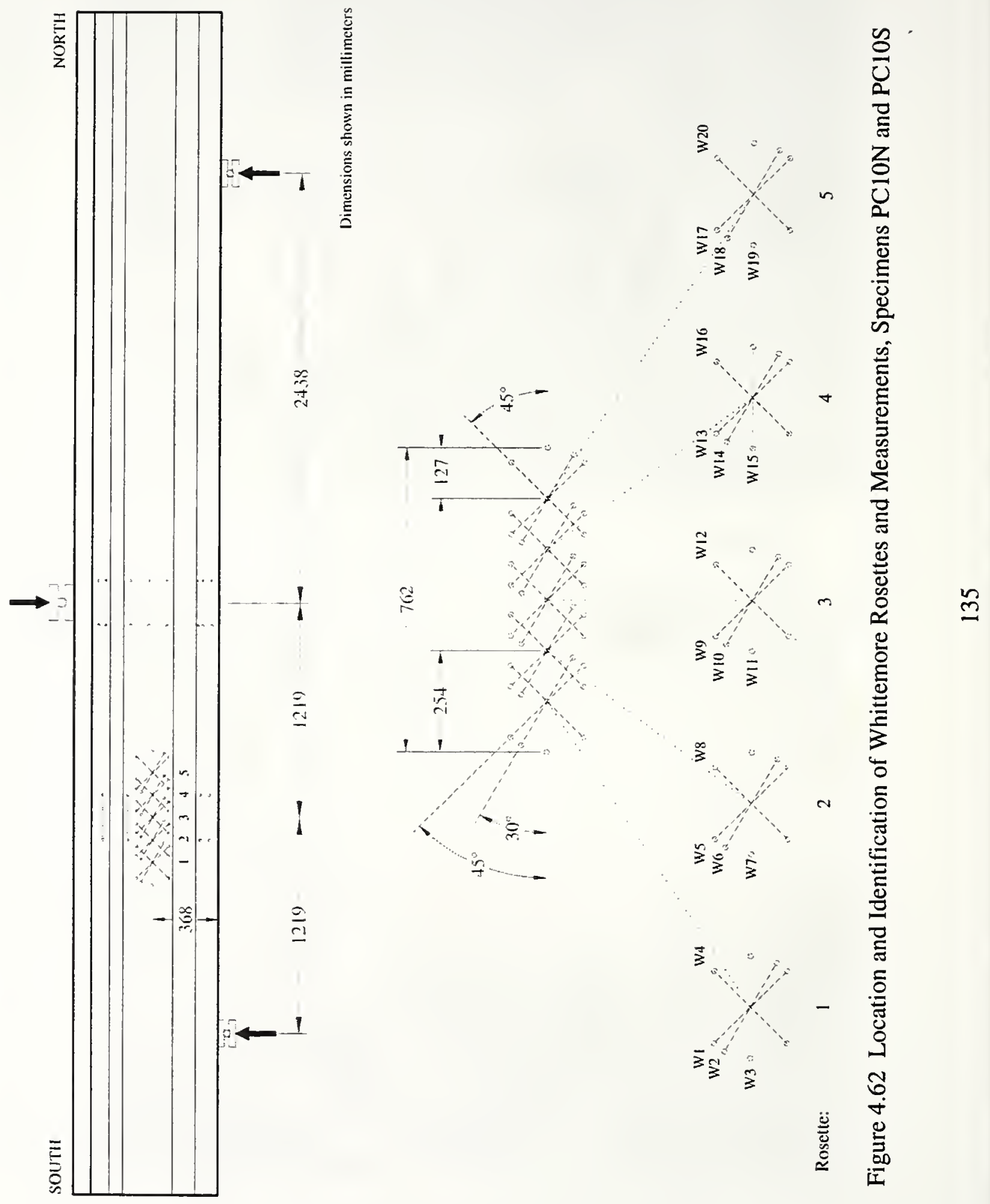


\subsection{Testing of Fresh Concrete Properties}

The fresh concrete properties of slump, unit weight and air content were determined for all the lightweight and normal weight concrete produced in the lab and in the precast plant. All fresh concrete properties were determined immediately after mixing. The specifications followed for testing fresh concrete properties are given in Table 5.1.

\subsubsection{Slump}

The slump of concrete was determined according to the procedure outlined in ASTM C 143. Typically, one slump measurement was performed per batch and it was conducted immediately after mixing before the mix was discharged from the mixer. The results for slump are presented in section 6.1.

\subsubsection{Unit Weight}

The procedure used was in accordance with ASTM C 138. Both $0.003 \mathrm{~m}^{3}$ and a $0.007 \mathrm{~m}^{3}\left(0.1\right.$ and $\left.0.25 \mathrm{ft}^{3}\right)$ unit weight containers were used in the determination of the unit weight of concrete depending on the maximum size and type of aggregate. The method of consolidation was by rodding since the slump of concrete was above $760 \mathrm{~mm}$ (3 in.). The concrete was placed in the container in three layers of approximately equal volume and each layer was rodded 25 times. The net weight of the concrete was then determined and the unit weight calculated. The results for unit weight are presented in section 6.1 . 


\subsubsection{Air Content}

The air content of lightweight concrete was determined by the volumetric meter (ASTM C 173). The air content of normal weight concrete was determined by the pressure meter (ASTM C 231). The pressure meter used was a Type B instrument consisting of a measuring bowl and cover assembly.

The air content measurements were taken immediately after mixing. One measurement of air content of concrete was made for every batch. The concrete used for air content measurement was not returned to the mixer.

\section{$\underline{5.2 \text { Testing of Hardened Concrete }}$}

The hardened concrete properties tested included compressive strength, flexural strength, tensile strength, static and dynamic modulus of elasticity, Poisson's ratio, and density. All concrete samples used in these tests were demolded after 24 hours and placed in the moist room until test time. The specifications followed while testing the hardened concrete properties are given in Table 5.2.

\subsubsection{Compressive Strength}

The compressive strength of concrete was determined using the method detailed in ASTM C 39. The concrete was made in $10 \mathrm{~cm} \times 20 \mathrm{~cm} \mathrm{(4} \mathrm{in.} \times 8$ in.) molds. The samples made in the field were demolded after 24 hours and transferred to the moist room for further curing. Compression testing of the $31 \mathrm{MPa}$ and $42 \mathrm{MPa}(4500 \mathrm{psi}$ and $6000 \mathrm{psi})$ concrete was done using a $45,359 \mathrm{~kg}(100,000 \mathrm{lb})$ capacity testing machine manufactured by Satec System, Inc. (Figure 5.1). Compression testing of the high 
strength concrete was done in using a $113,398 \mathrm{~kg}$ (250,000 lbs) capacity testing machine manufactured by Forney Testing Equipment.

The compressive strength recorded was an average of three concrete samples. The compression testing was done in most cases at 7, 14, and 28 days. In cases where strength data was needed for the purpose of determining the strand release time, additional compression testing was done at 1 day. The concrete mixes containing fly ash were also tested at 56 days. The results for compressive strength are presented in section 6.2 .1 .

\subsubsection{Flexural Strength}

The flexural testing was done according to ASTM C 78 using the simple beam with third point loading. The concrete samples for determining flexural strength were 13 $\mathrm{cm} \times 13 \mathrm{~cm} \times 48 \mathrm{~cm}(6$ in. $\times 6$ in. $\times 22$ in.) in dimension (Figure $5.2-5.3$ ). Results were obtained from an average of three samples in section 6.2.2.

\subsubsection{Split Tensile Strength}

The tensile strength of concrete was conducted according to ASTM C 496. The concrete samples were made using $13 \mathrm{~cm} \times 26 \mathrm{~cm}$ (6 in. $\times 12$ in.) molds. The splitting tensile strength of the concrete was determined from an average of three samples. The concrete samples were demolded after 24 hours and placed in the moist room until testing at 28 days (Figure 5.4). The results for split tension strength are presented in section

\subsection{3.}




\subsubsection{Static and Dynamic Modulus of Elasticity, Poisson' Ratio}

The ASTM C469 method was used to determine the static modulus of elasticity and Poisson's Ratio. The concrete samples were $15 \mathrm{~cm} \times 30 \mathrm{~cm}$ (6 in. $\times 12$ in.) in dimension. The concrete samples were demolded after 24 hours and placed in the moist room until test time. A combined compressometer-extensometer setup was used to determine the longitudinal and transverse strains (Figures 5.5 and 5.6).

As required by ASTM C469 the compressive strength of concrete was determined first and samples were loaded to $40 \%$ of that value. The modulus of elasticity samples were pre-loaded without taking any readings and were then loaded two more times during which the values of load, and longitudinal and transverse strains were recorded. The static modulus of elasticity and Poisson's Ratio were determined from an average of 3 readings taken from one sample and the results are presented in section 6.2.4.

The dynamic modulus of elasticity was determined using a Grindo-Sonic nondestructive materials testing system (Figure 5.7). The concrete samples had dimensions of $7.6 \mathrm{~cm} \times 7.6 \mathrm{~cm} \times 38 \mathrm{~cm}$ ( 3 in. $\times 3$ in. $\times 15$ in.). The concrete samples were demolded after 24 hours and placed in the moist room until test time. A sensor pin connected to the Grindo-Sonic machine was placed on the side of the sample and a light plastic hammer was used to produce transverse waves. A frequency reading was recorded of the display panel. Three readings of frequency were recorded and averaged for use in calculation of dynamic modulus of elasticity. Values of weight, dimensions, and frequency were imputed into the computer program that comes with the Grindo-Sonic machine and the dynamic modulus was calculated. The dynamic modulus of elasticity was determined using one sample and the results are presented in section 6.2.4. 


\subsubsection{Density of Hardened Concrete}

The concrete samples used for density measurements were $10 \mathrm{~cm} \times 20 \mathrm{~cm}(4 \mathrm{in}$. $\mathrm{x}$ 8 in.) in dimension. The samples were demolded after 24 hours and cured for 28 days in the moist room. The samples were then air dried in the lab for another 28 days. The weight of the concrete samples were then determined and the density calculated. The density of concrete was determined from an average of three samples. The results for density of hardened concrete are presented in section 6.2.5.

\subsubsection{Temperature Development}

Because of the high cement content in the $69 \mathrm{MPa}(10,000 \mathrm{psi}) \mathrm{LWC}$ mix, there were concerns about thermal cracking and temperature development evaluation was done only for that mix. The setup for temperature development evaluation consisted of a data logger (CR10 X, Campbell Scientific Inc.) that had thermocouples connected. The data logger collected time and temperature data for a period of three days starting after initial casting. Readings were recorded every five minutes during testing. The thermocouples were placed inside the concrete molds. The molds were placed inside a styrofoam container which had small styrofoam packing around the molds for the purpose of insulation.

The concrete samples used were $15 \mathrm{~cm} \mathrm{x} 30 \mathrm{~cm}$ (6 in. $\times 12$ in.) in dimensions. Two concrete samples were prepared inside styrofoam container. A control sample was prepared and cured in air. The insulated sample simulated the curing conditions inside the beams cast in the field. The results for temperature development in the $69 \mathrm{MPa}$ (10,000 psi) LWC are presented in section 6.2.6. 


\subsection{Testing of Durability Properties of Concrete}

Air void distribution, drying shrinkage, freeze-thaw resistance, chloride ion permeability and resistance to scaling tests were conducted on both NWC and LWC produced in the lab. Air void distribution and resistance to scaling tests were performed only on the deck concrete. Table 5.2 outlines the specifications followed during testing of durability properties.

\subsubsection{Air Void Distribution}

The modified point-count method was used to evaluate the air void system in hardened concrete (ASTM C457). Concrete samples of dimensions $7.6 \mathrm{~cm}$ x $15 \mathrm{~cm}$ (3 in. $x 6$ in.) were prepared. The samples were demolded after 24 hours and placed in the moist room until test time. The samples were sawed in half along the long axis and their surface polished using progressively finer abrasives (No. 100, 180. 240,600, 800 and 1000) until the surface was suitable for microscopic observation. All surfaces were washed and scrubbed thoroughly after sawing. Measurements were made on one sample for each mix.

The point-count device had a platform connected to the lead screw and was constructed in a way that the concrete sample placed on the platform was moved smoothly and uniformly through equal distances by turning the lead screw (Figure 5.8). A Microscope was used to view the polished surface at a magnification of 50X. A counter was used to tally air, paste, fine aggregate, coarse aggregate and intercepted air voids that were traversed under the microscope. Air void, air void-paste ratio, and 
spacing factor were calculated. Results for air -void distribution are presented in section 6.3.1.

\subsubsection{Drying Shrinkage}

The determination of length change of concrete specimens was conducted in accordance with ASTM C 490 and C 157. The molds used to manufacture the tests specimens had dimensions of the $7.6 \mathrm{~cm} \times 7.6 \mathrm{~cm} \times 29 \mathrm{~cm}(3$ in. $\times 3$ in. $\times 11.25$ in.) having a gage length of $25 \mathrm{~cm}$ (10 in). A total of four samples were prepared for shrinkage determination. The gage studs used were manufactured by Humboldt company (H-3260). The length comparator for determining drying shrinkage had a dial micrometer with a smallest division of 0.00025-cm (0.0001-in).

Concrete samples were batched, placed in the moist room, and demolded after 24 hours of storage. They were then placed in lime-saturated water for 30 minutes after which they were taken out and dried with a cloth. The initial comparator reading was then taken. The samples were returned to the lime saturated water until test time. After 7 days of curing in lime saturated water, half of the samples for each concrete mix were removed from the water and air cured in the lab until 28 days when the final comparator reading was taken. The results for drying shrinkage are presented in section 6.3.2.

\subsubsection{Freeze-Thaw Resistance}

The freezing and thawing resistance of concrete was tested according to ASTM C666 method procedure A. The concrete samples were $7.6 \mathrm{~cm} \mathrm{x} 7.6 \mathrm{~cm} \times 38 \mathrm{~cm}(3 \mathrm{in} . \mathrm{x}$ 3 in. $x 15$ in) beams. Two samples were tested for each mix. A total of 17 different 
mixes were evaluated. These mixes were divided in 3 categories: Girder mixes, newly developed deck mixes, and reference deck mixes.

Concrete samples from girder mixes were cast and cured in the moist room for 24 hours. They were then demolded and returned to the moist room for further curing. At 14 days weight and dynamic modulus of elasticity of the samples were determined and the samples placed in the freeze-thaw chamber and subjected to freezing and thawing cycles (Figure 5.9). Thereafter, measurements of weight and dynamic modulus of elasticity were taken weekly (approximately every 30 cycles) for the duration of the test. The test was terminated after 300 cycles.

Initially, all samples for deck concrete were cured the same way as samples for girder concrete. However, the initial freeze-thaw results obtained for LWC were unsatisfactory. The LWC containing silica fume failed after about 72 cycles of freezing and thawing and the concrete with no mineral admixture faired just a little better. The concrete containing fly ash showed overall the best results although it did not pass the test. The existing data on freeze-thaw performance of LWC indicated that it may be beneficial to dry samples prior to subjecting them to freezing and thawing cycles. The deck concrete mixes were retested using modified curing procedure that included 14 days of moist curing followed by 14 days of air drying prior to freezing and thawing cycles. The reference deck concrete mixes were tested following this modified curing procedure. The results for freeze-thaw resistance are given in section 6.3.3.

The durability factors obtained for the concrete samples that were air-dried were significantly better. The author is of the opinion that the modified procedure for curing LWC samples is justifiable as it better represents the field conditions. In field application 
the concrete decks are never poured after the first frost and the concrete is thus allowed to dry out and develop sufficient compressive strength before subjected to any freezing and thawing cycles. If the samples were not allowed to dry, the considerable moisture trapped in the LWA undergoes expansion and contraction during freezing and thawing and causes rapid deterioration of concrete. This rapid deterioration of concrete was not observed for bridge girders because this concrete had higher strengths at 14 days and was thus able to withstand the harsh conditions of the freezing and thawing test.

\subsubsection{Rapid Chloride Ion Permeability}

The testing was done in accordance with ASTM C 1202. This test evaluates the electrical conductance of concrete samples to provide a rapid indication of their resistance to chloride ion penetration. The test samples were cut from $10 \mathrm{~cm} \times 20 \mathrm{~cm}$ (4 in. $x 8$ in.) cylinders that were moist cured. Measurements of chloride ion permeability were performed on two concrete samples. Tests were done at 28 and 56 days for bridge girder concrete, and 28, 56, and 90 days for bridge deck concrete (Figure 5.10).

On the specified day of testing, the samples were removed from the moist room and allowed to dry for 8 hours. Devon Polystrate 2 Ton Epoxy was used to completely coat the sides of the samples. The samples were then allowed to cure for at least 3 hours and were then placed in a plastic container. The plastic container was then placed in a vacuum dessicator. The dessicator was sealed with sealant grease and the vacuum pump started. The vacuum was maintained for 3 hours.

With the pump running, de-aerated water was introduced into the plastic container to cover the samples. The water stopcock was closed and the vacuum pump allowed to 
run for another hour. At the end of this period the samples were removed from the vacuum system and were kept in the water for an additional 18 hours. Silicone sealant was used to mount the specimen to the cells. The assembled cells were left for 2 hours to allow the sealant to cure. The cells were then filled with $3 \% \mathrm{NaCl}$ and $0.3 \mathrm{~N} \mathrm{NaoH}$ solutions and the lead wires were attached to the cell electrical connections (Figure 5.9). The coulomb and current readings were taken every 30 minutes. The test was automatically terminated after 6 hours. The results of rapid chloride ion permeability are presented in section 6.3.4.

\subsubsection{Resistance to Scaling}

The scaling resistance of concrete was performed in accordance with ASTM C 672 procedure except that $3 \%$ sodium chloride was used instead of anhydrous calcium chloride. Concrete samples of dimensions $7.6 \mathrm{~cm} 25 \mathrm{~cm} \mathrm{x} 19 \mathrm{~cm}$ ( 3 in. $x 10$ in. $x 7.5$ in.) with a surface area of $484 \mathrm{~cm}^{2}\left(75 \mathrm{in}^{2}\right)$ were cast and moist cured for 14 days. After 14 days the samples were removed from the moist room and stored in a constant temperature and relative humidity chamber $\left(20^{\circ} \mathrm{C}\right.$ and $\left.50 \%\right)$ for an additional 14 days. Roofing rubber material was used to construct a $5 \mathrm{~cm}(2 \mathrm{in}$.) dike around the perimeter of the top surface of the samples. The samples were then subjected to 50 cycles of freezing and thawing in a freezing chamber (Figure 5.11). The freezing cycle lasted 16 hours and was followed by 8 hours of thawing. The results for resistance to scaling of concrete are presented in section 6.3.5.

A small modification to ASTM C 672 was made which included thawing the samples in the freezer chamber that was adjusted to room temperature rather than 
removing the samples from the freezing chamber and thawing them in the lab. Although this may have led to samples being exposed to a slightly higher relative humidity it is not expected that this deviation from the standard would have any significant influence on the scaling resistance.

Water was added daily to maintain the proper depth of solution. Every five cycles the surface was thoroughly flushed and a visual examination made. The solution was replaced and the test continued.

\section{$\underline{5.4 \text { Shear Tests }}$}

\subsubsection{Reinforced Concrete Specimens}

\subsubsection{Loading Frame}

The test setup for the reinforced concrete specimens is shown in Figures 5.12 and 5.13. Rollers at the two support locations supported the specimen. The support beams were built-up steel sections composed of two vertically stiffened channels welded to plate steel. Two load cells placed undermeath supported each support beam. Two Lebow Model 3156-150K load cells were underneath the south support. One Lebow Model 3156-100K and one Lebow Model 3156-150K were underneath the north support. The $3156-100 \mathrm{~K}$ and $3156-150 \mathrm{~K}$ load cells had capacities of $445 \mathrm{kN}$ and $667 \mathrm{kN}$, respectively. Figure 5.14 shows the support beam and load cells at the north end of the test setup.

The load was applied to the steel spreader beam by a $2670 \mathrm{kN}$ Baldwin testing machine. The spreader beam distributed two thirds of the total load to the north end (as shown in Figure 5.15) and one third of the total load to the south cantilever. The 
specimens were loaded to produce a constant shear force and reverse bending in the test region, as shown in Figure 5.16. This loading resulted in a more critical shear condition for the test region of the beam than does a concentrated load at midspan of a simply supported beam with the same a to $d(\mathrm{M} / \mathrm{Vd})$ ratio. The shear capacity associated with arch action is lesser in the selected test layout.

\subsubsection{Testing Program}

Prior to loading, measurements were taken for all instrumentation. The loading rate was controlled manually with the valves on the Baldwin testing machine. Loading was stopped and the current load held constant at every 44 to $89 \mathrm{kN}$ to record dial gage measurements and to trace the crack pattern. Measurements were recorded from the LVDTs, electrical resistance strain gages, and load cells approximately every $5 \mathrm{kN}$ of total applied load. Most tests lasted between 6 and 8 hours. Specimen 4-NWLD took 11 hours to test, as this was the first beam to be tested.

\subsubsection{Prestressed Concrete Specimens}

\subsubsection{Loading Frame}

The specimens were simply supported and subjected to a single point load at midspan, as shown in Figures 5.17 and 5.18. Rollers at the two support locations supported the specimen. The support beams were built-up steel sections composed of two vertically stiffened channels welded to plate steel. Two load cells placed underneath supported each support beam. Two Lebow Model 3156-150K load cells were underneath the south support. One Lebow Model 3156-100K and one Lebow Model 3156-150K 
were undermeath the north support. The 3156-100K and 3156-150K load cells had capacities of $445 \mathrm{kN}$ and $667 \mathrm{kN}$, respectively. Figure 5.19 shows the support beam and load cells at the south end of the test setup.

The load was applied to the specimen by a $2670 \mathrm{kN}$ Baldwin testing machine. A $203 \mathrm{~mm} \times 356 \mathrm{~mm}$ bearing plate distributed the applied load to the top surface of the slab, as shown in Figure 5.19.

\subsubsection{Testing Program}

Prior to loading, measurements were taken for all instrumentation. The loading rate was controlled manually with the valves on the Baldwin testing machine. Loading was stopped and the current load held constant at every 44 to $67 \mathrm{kN}$ to record dial gage measurements and to trace the crack pattern. Measurements during the test included loads at the supports, deflections at the supports, midspan, and quarterpoints, and strains in the stirrups, prestressing strand, mild tension reinforcement, and mild compression reinforcement in the slab. Measurements were recorded approximately every $5 \mathrm{kN}$ of total applied load. The tests lasted between 4 and 8 hours. 
Table 5.1 Specifications Followed During Testing of Fresh Concrete Properties

\begin{tabular}{|c|c|}
\hline Test & Fresh Concrete Properties \\
\hline Slump & ASTM C 143 \\
\hline Air Content & ASTM C173, C231 \\
\hline Unit Weight & ASTM C 138, C 567 \\
\hline
\end{tabular}

Table 5.2 Specifications Followed During Testing of Hardened Concrete Properties

\begin{tabular}{|c|c|}
\hline Test & Hardened Concrete Properties \\
\hline Compressive Strength & ASTM C 39 \\
\hline Flexural Strength & ASTM C 78 \\
\hline Tensile Splitting & ASTM C 496 \\
\hline Static/Dynamic Modulus & ASTM C 469 \\
\hline Poisson's Ratio & ASTM C 469 \\
\hline Rapid Chloride Permeability & ASTM C 1202 \\
\hline Freezing and Thawing & ASTM C666 (Procedure A) \\
\hline Drying Shrinkage & ASTM C157, C 490 \\
\hline Resistance to Scaling & ASTM C 672 \\
\hline Temperature Development & \\
\hline Air Void Distribution & ASTM C457 \\
\hline
\end{tabular}




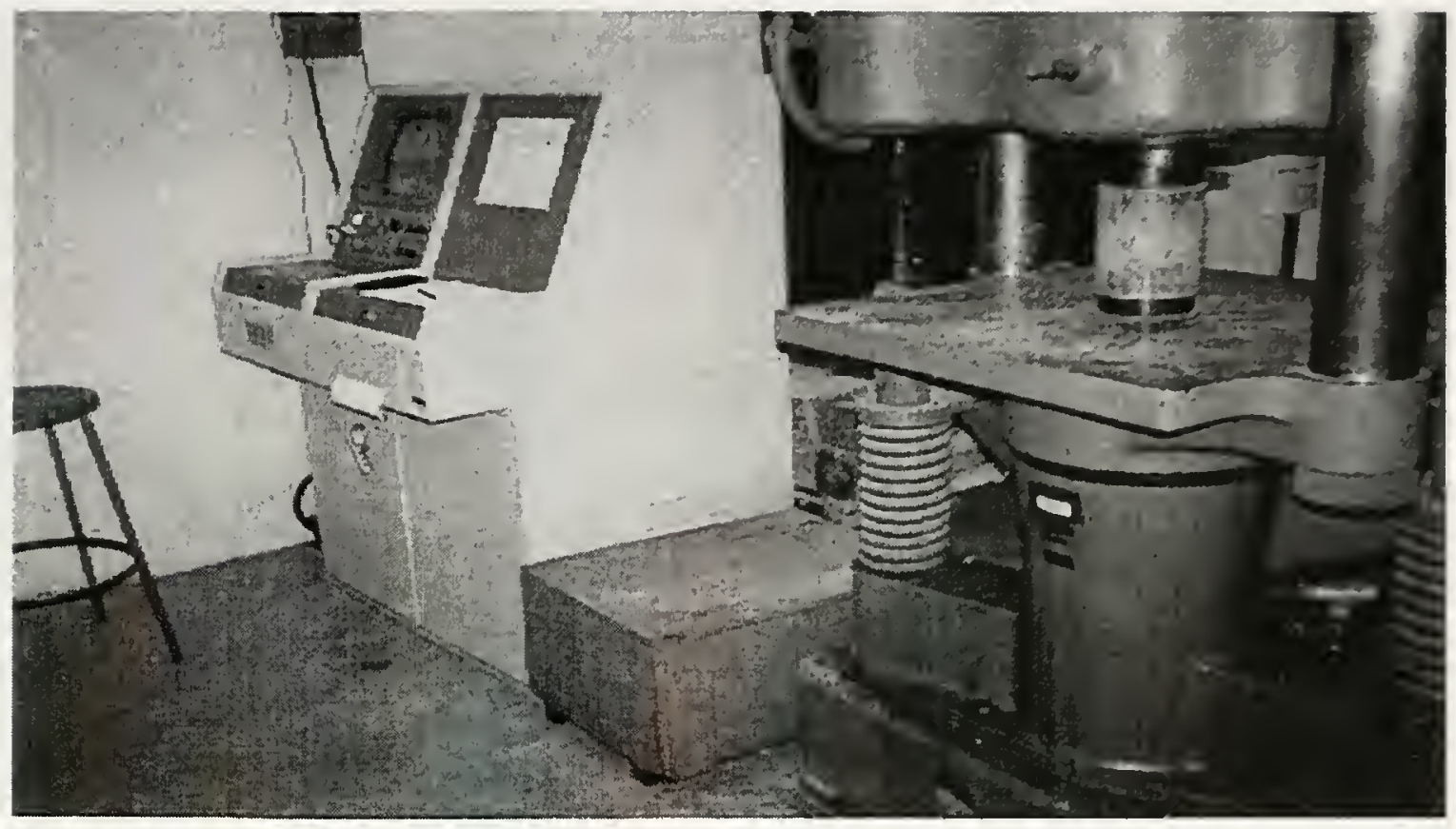

Figure 5.1 Compression Testing of Concrete Cylinders

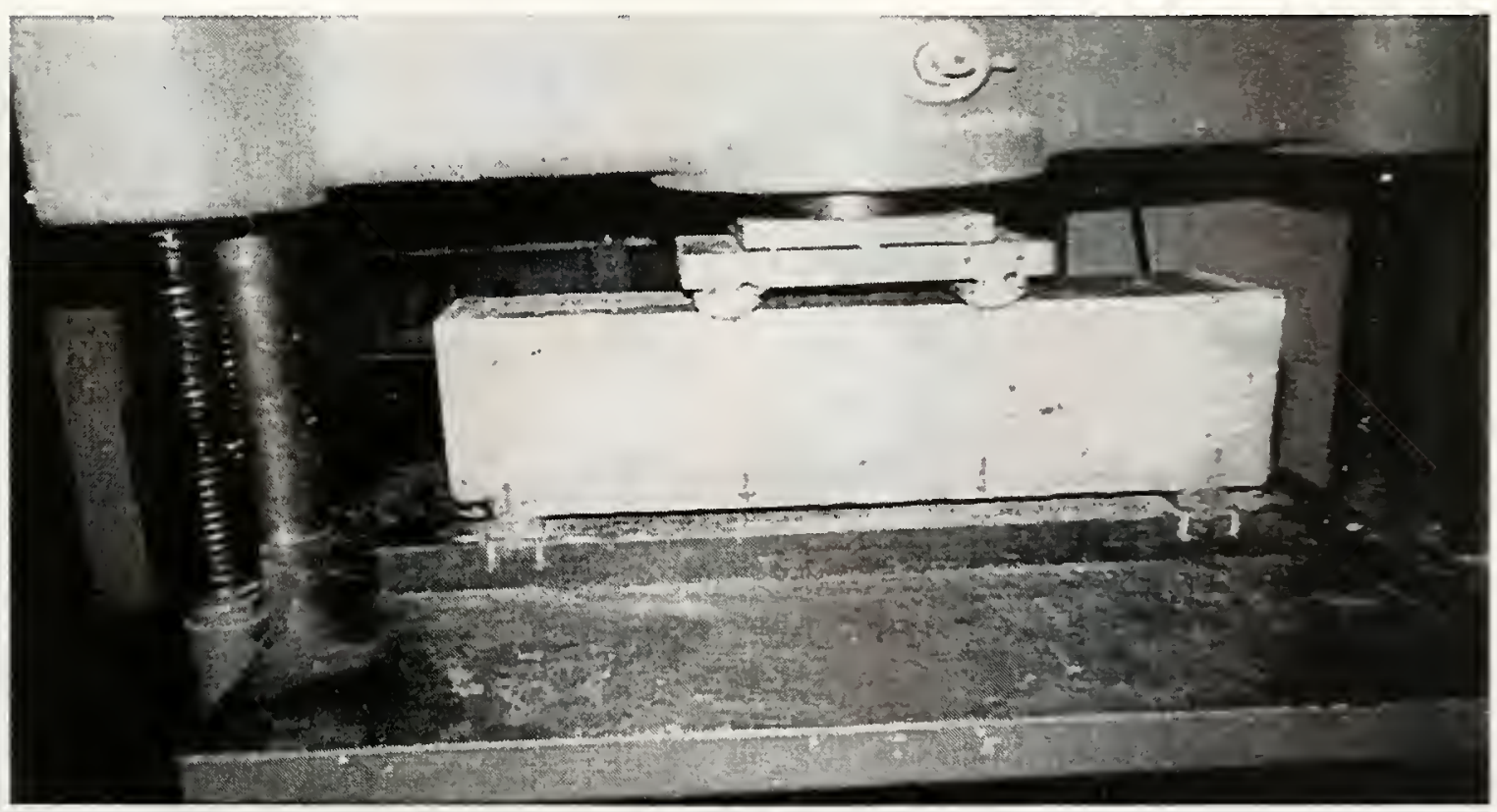

Figure 5.2 Concrete Beam being Loaded by The Simple Third Point Method 


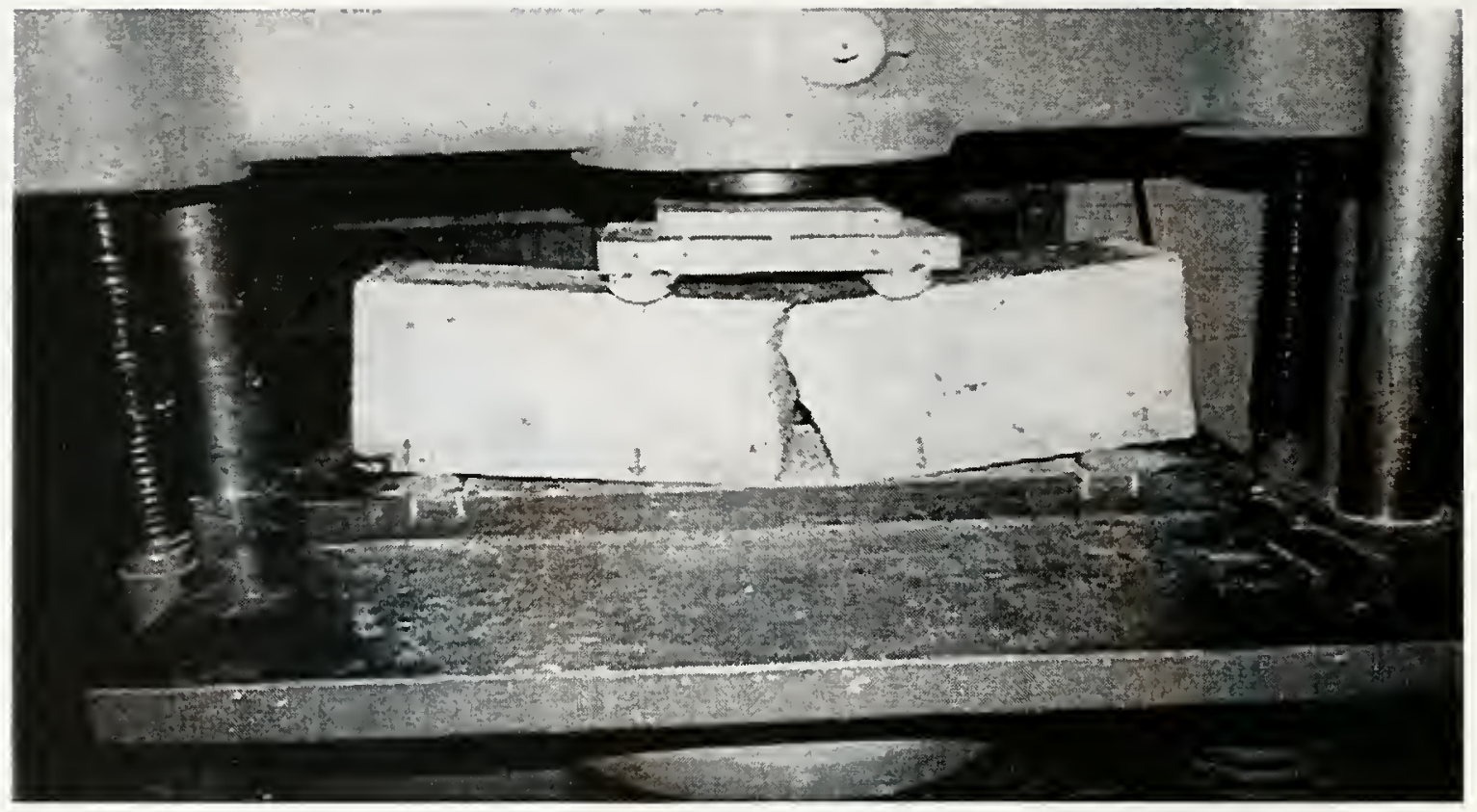

Figure 5.3 Failure of Concrete Beam in Flexure

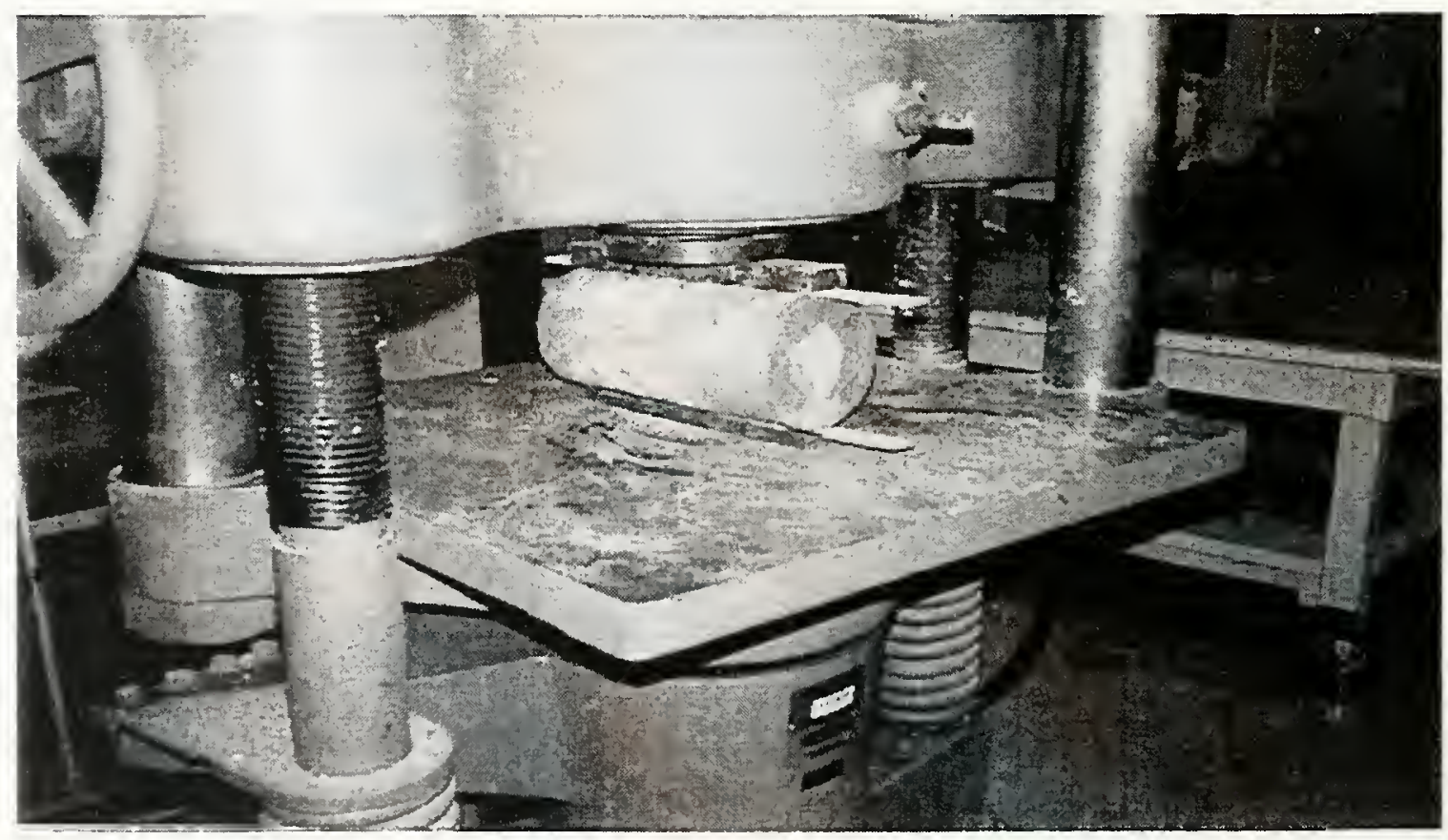

Figure 5.4 Tensile Splitting Test of Concrete Sample 


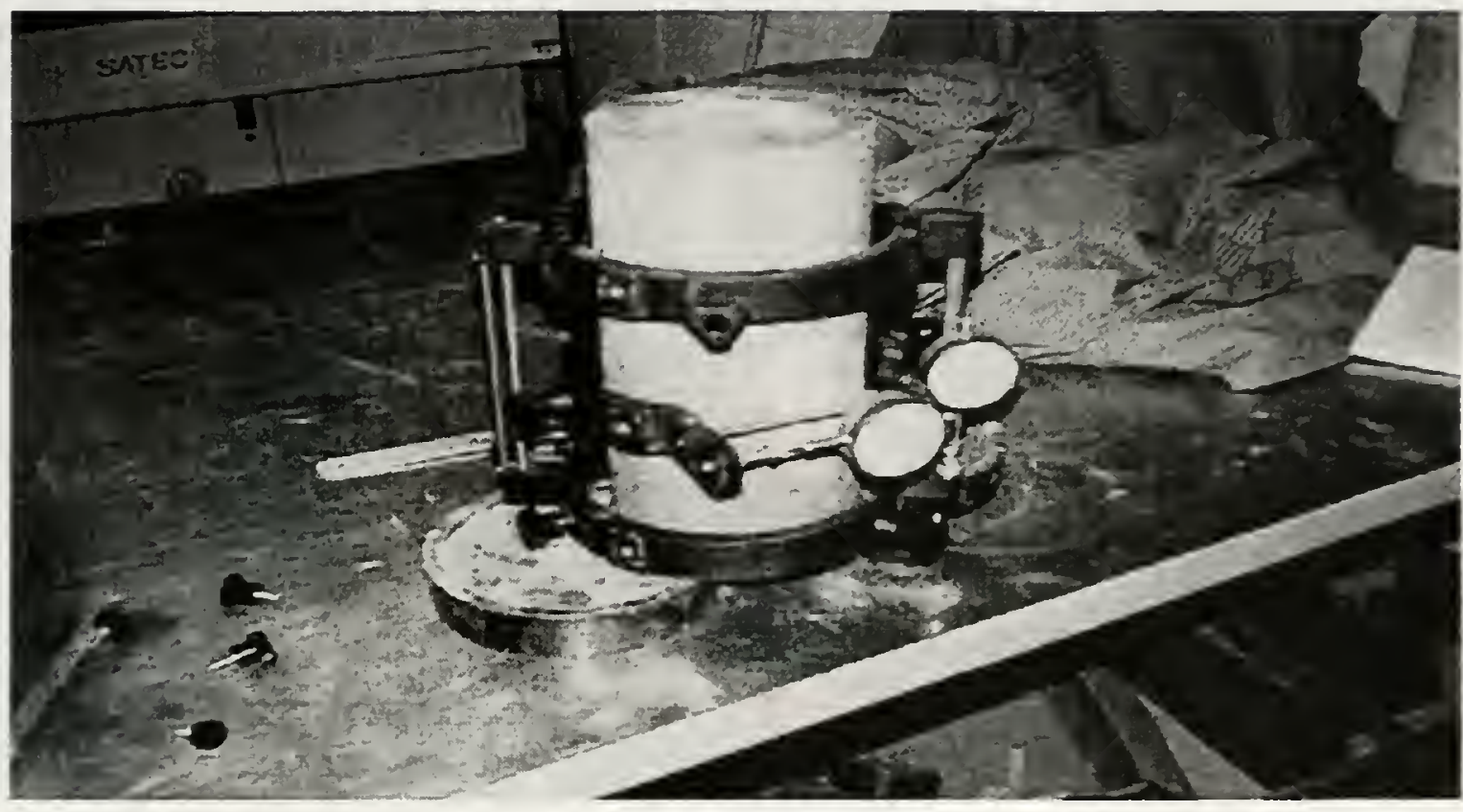

Figure 5.5 Test Setup for Static Modulus of Elasticity and Poisson's Ratio

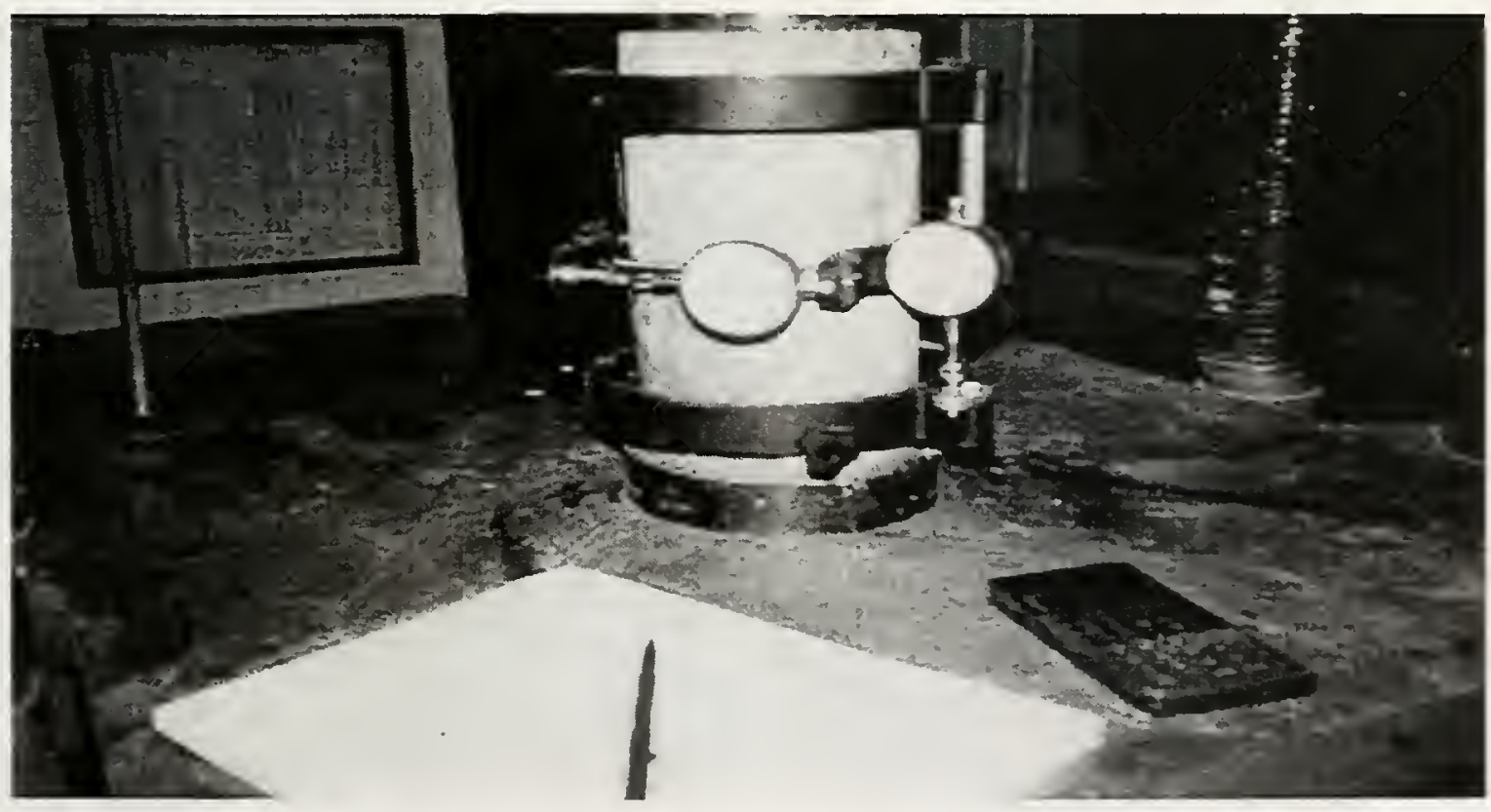

Figure 5.6 Testing for Static Modulus of Elasticity and Poisson's Ratio 


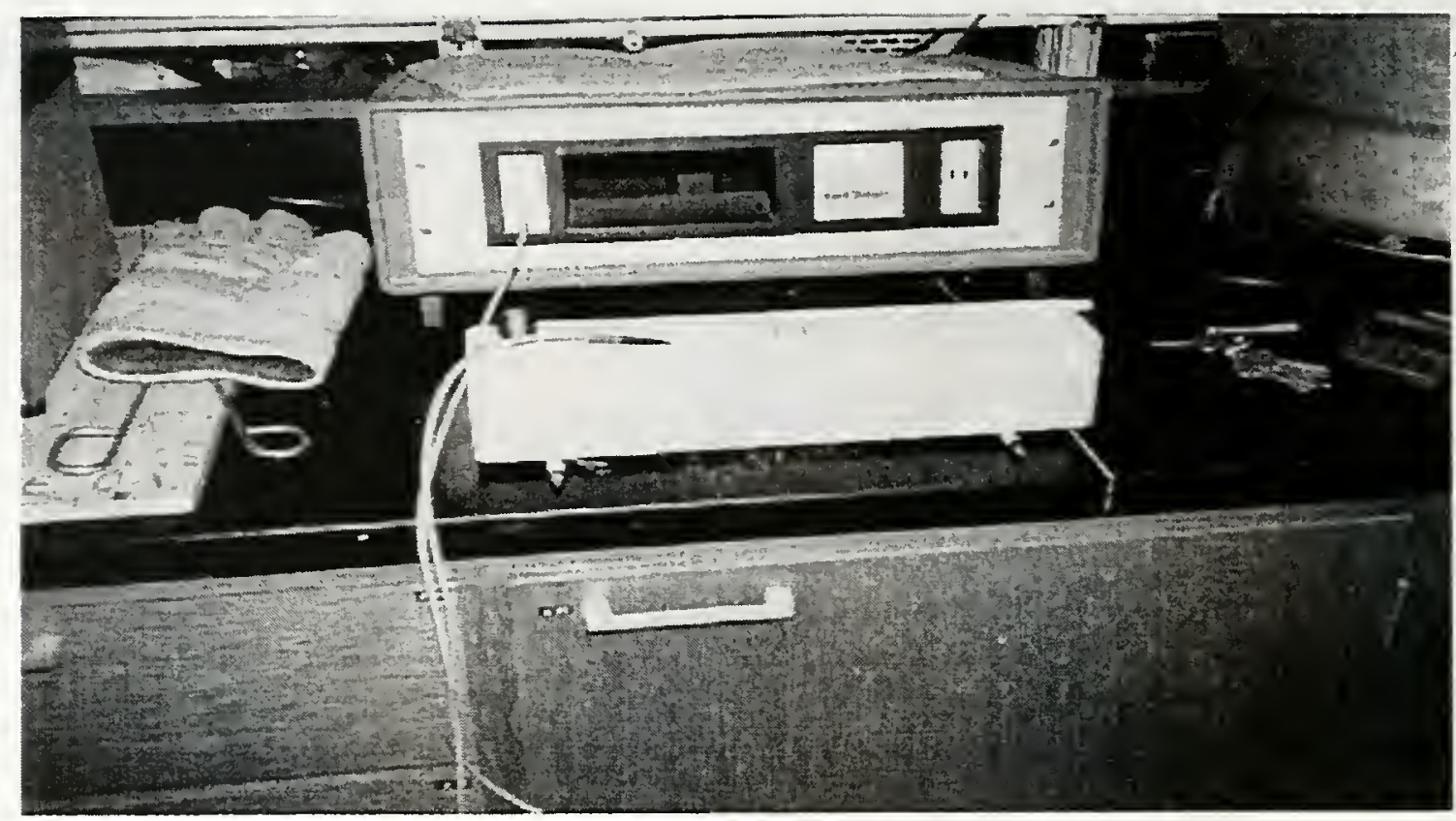

Figure 5.7 Testing of $6.6 \mathrm{~cm} \times 6.6 \mathrm{~cm} \times 33 \mathrm{~cm}$ (3in. x 3in. x 15 in.) Beams for Dynamic Modulus of Elasticity Using Grindo-Sonic Non-destructive Materials Testing System.

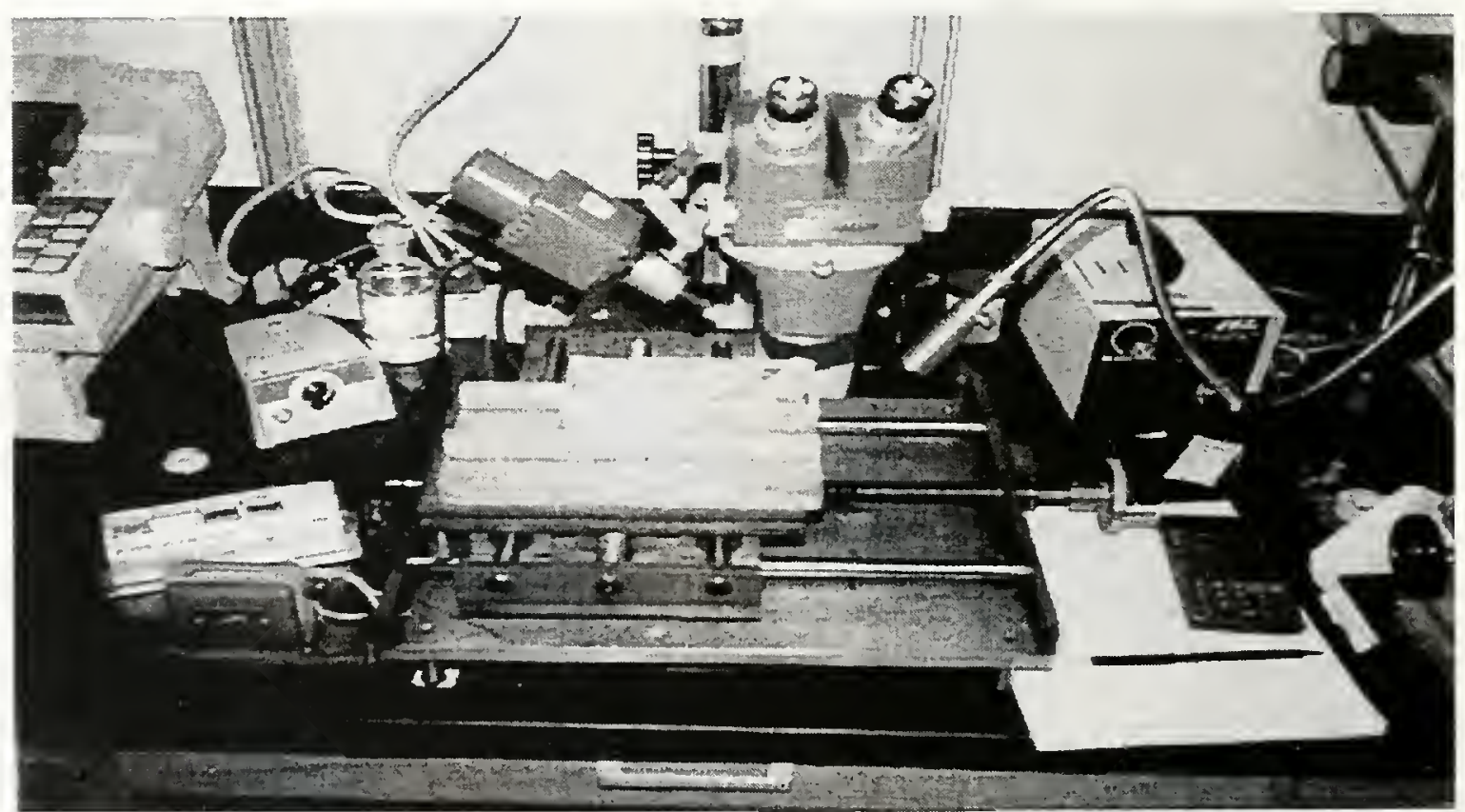

Figure 5.8 Air Void Distribution Determination Using Modified Point Count Method 


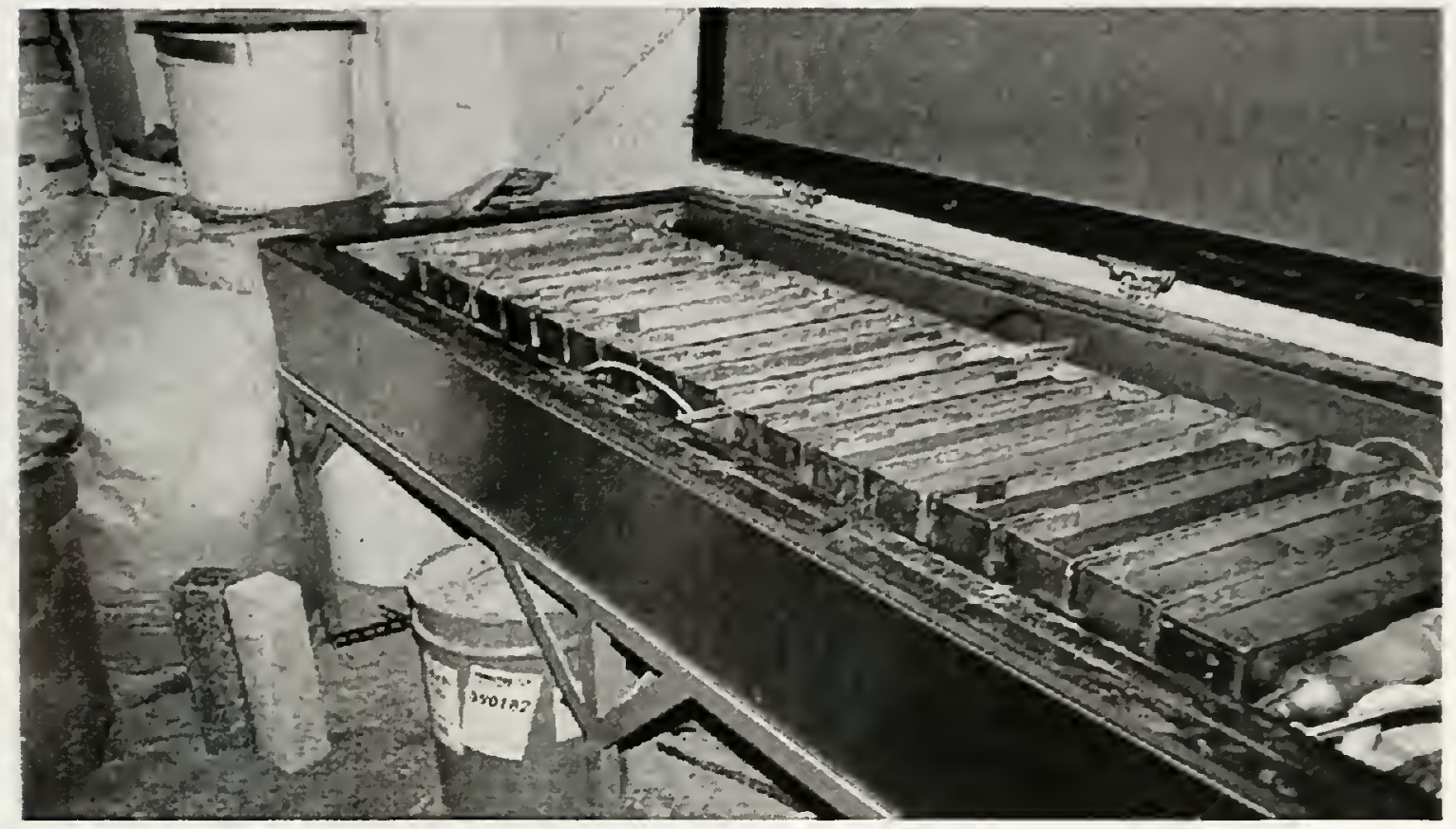

Figure 5.9 Testing of $6.6 \mathrm{~cm} \times 6.6 \mathrm{~cm} \times 33 \mathrm{~cm}$ (3in. x 3in. x 15 in.) Beams in Freezing and Thawing Chamber

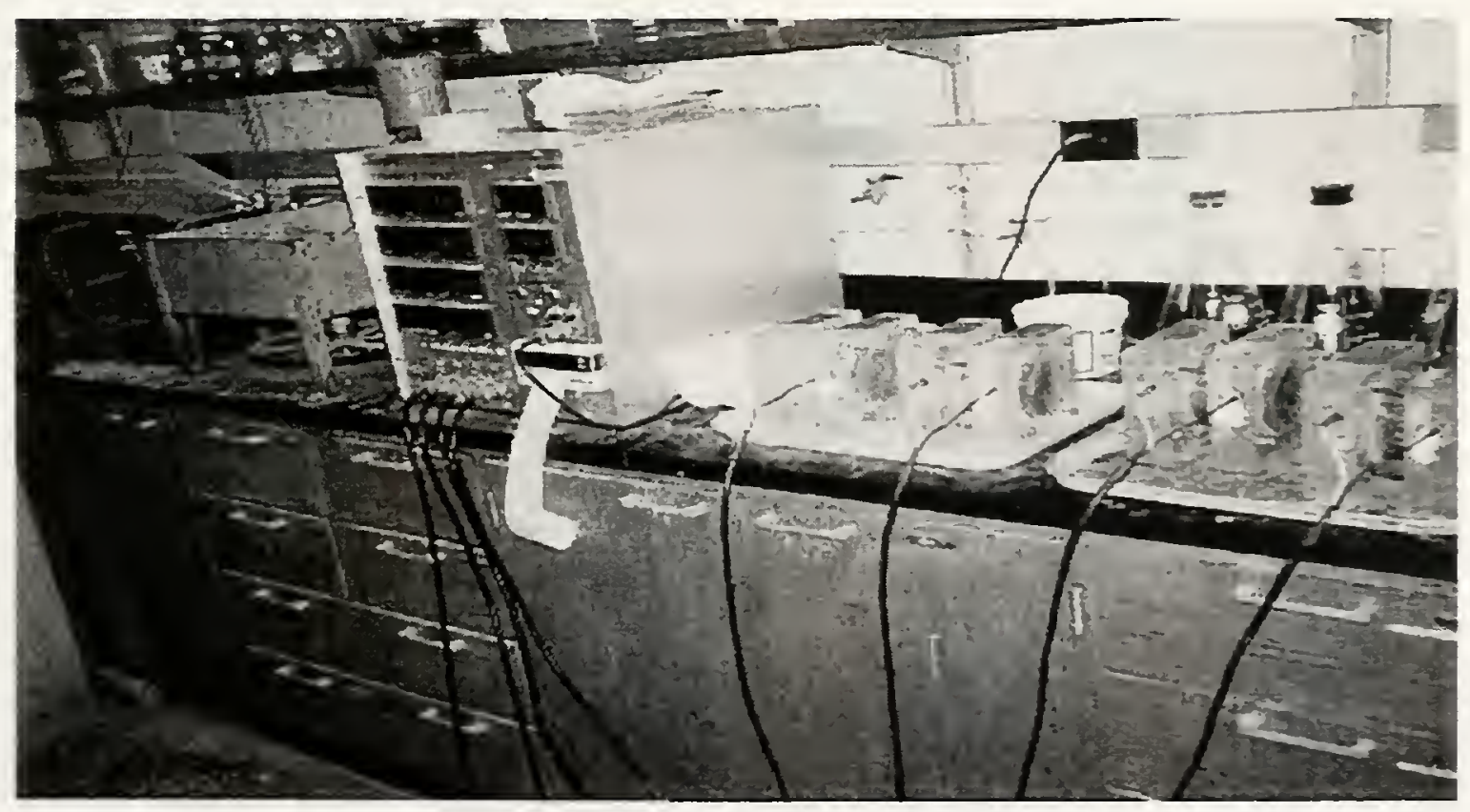

Figure 5.10 Rapid Chloride Ion Permeability Testing of Concrete Prisms 


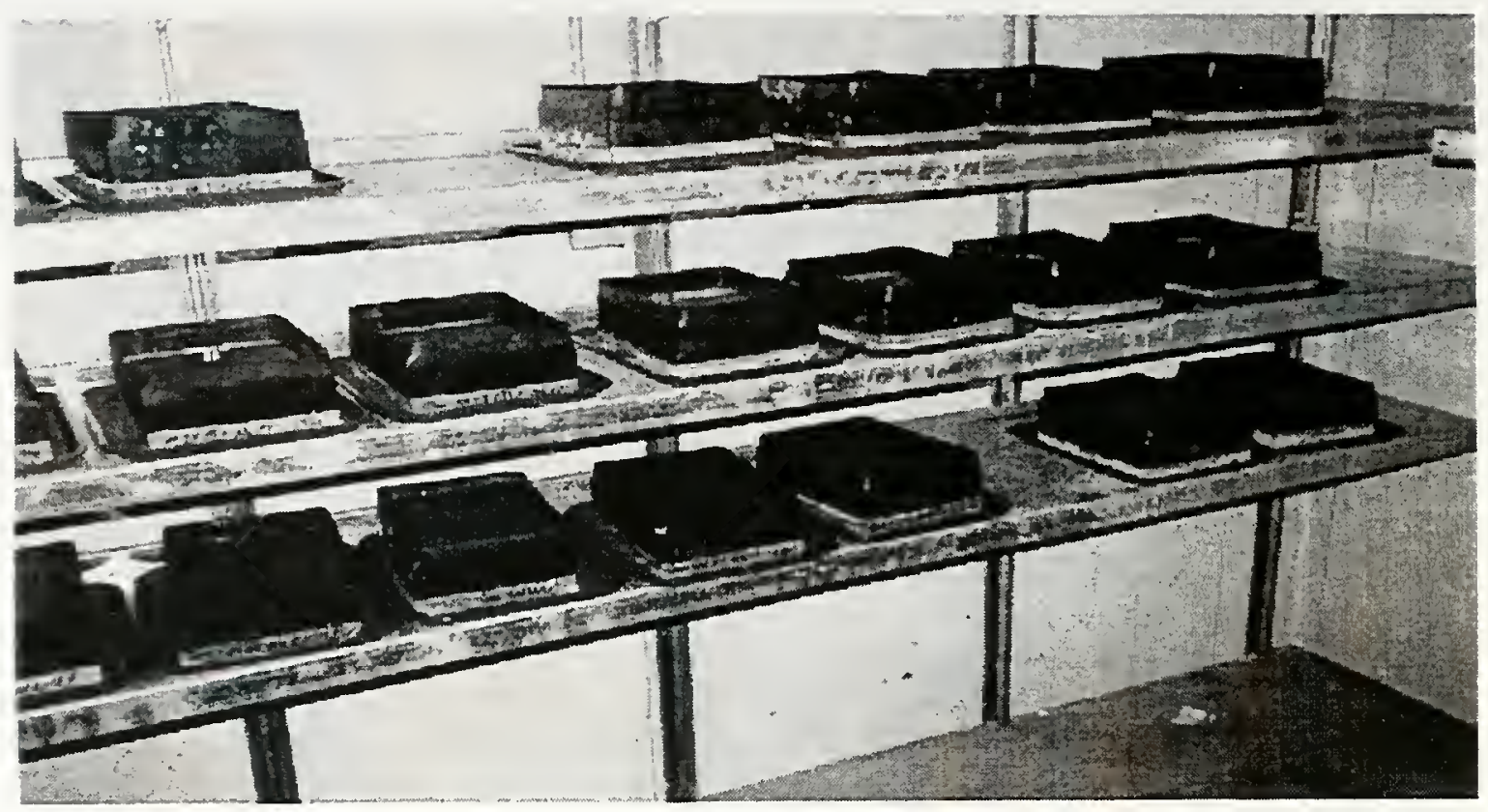

Figure 5.11 Resistance to Scaling Testing of Concrete Samples in Freezing Chamber 

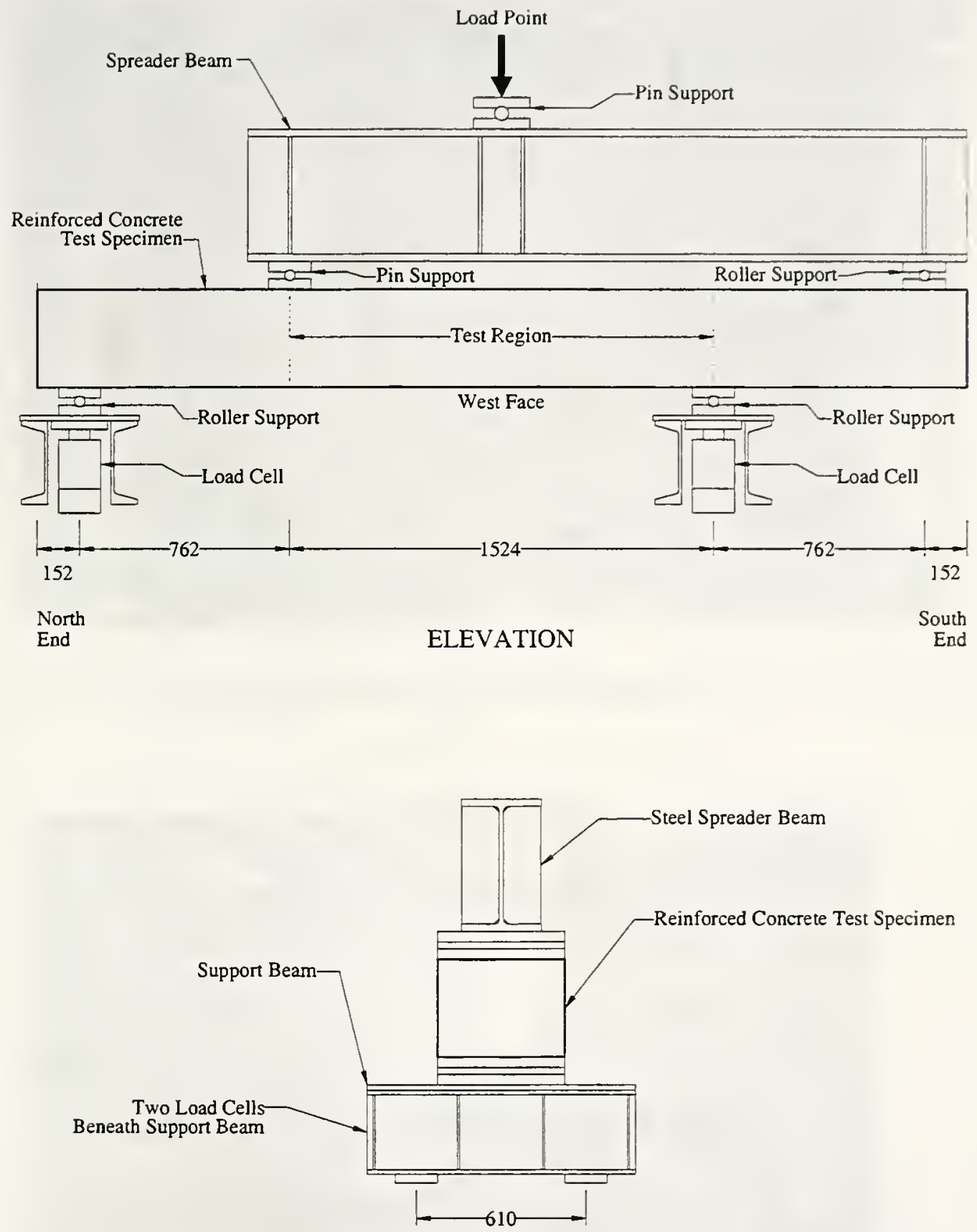

CROSS SECTION AT SUPPORT

Dimensions shown in millimeters.

Figure 5.12 Test Setup for Reinforced Concrete Series 


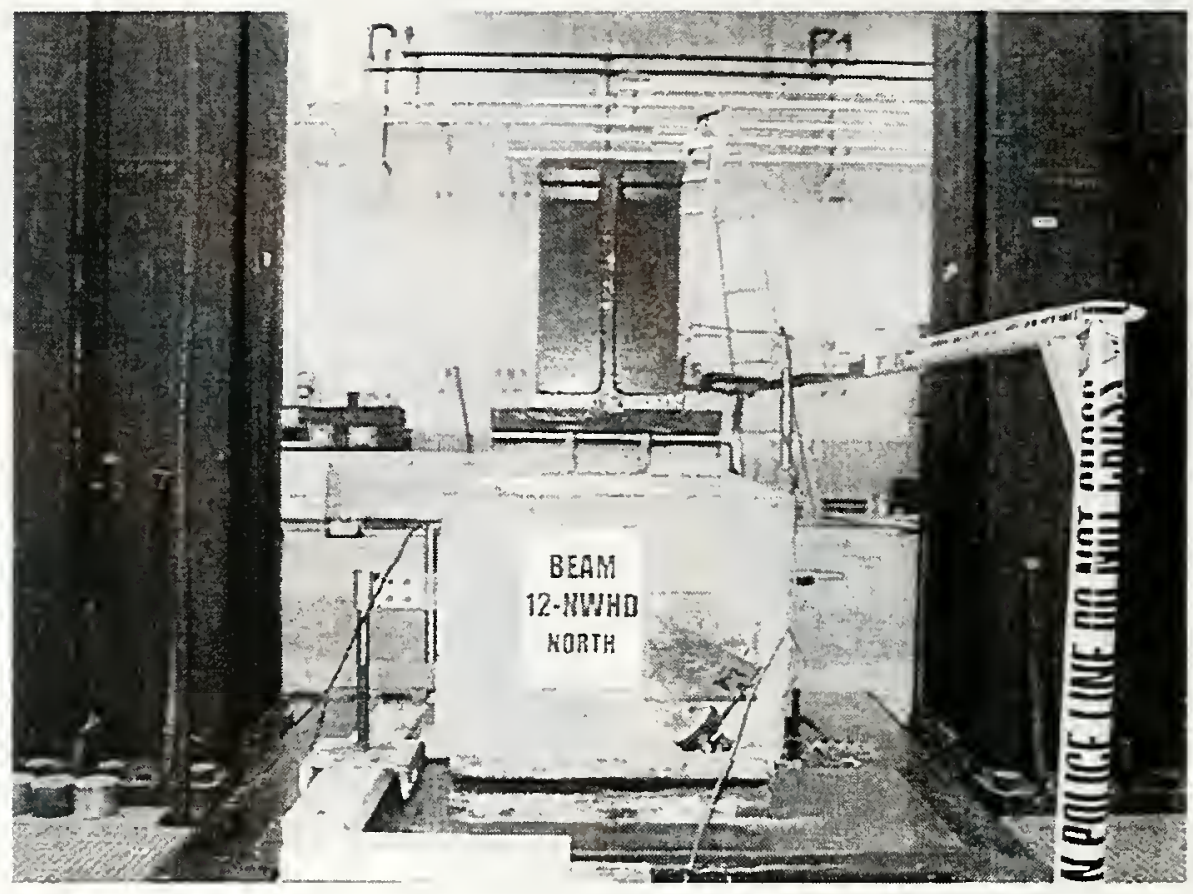

Figure 5.13 North End of Test Setup

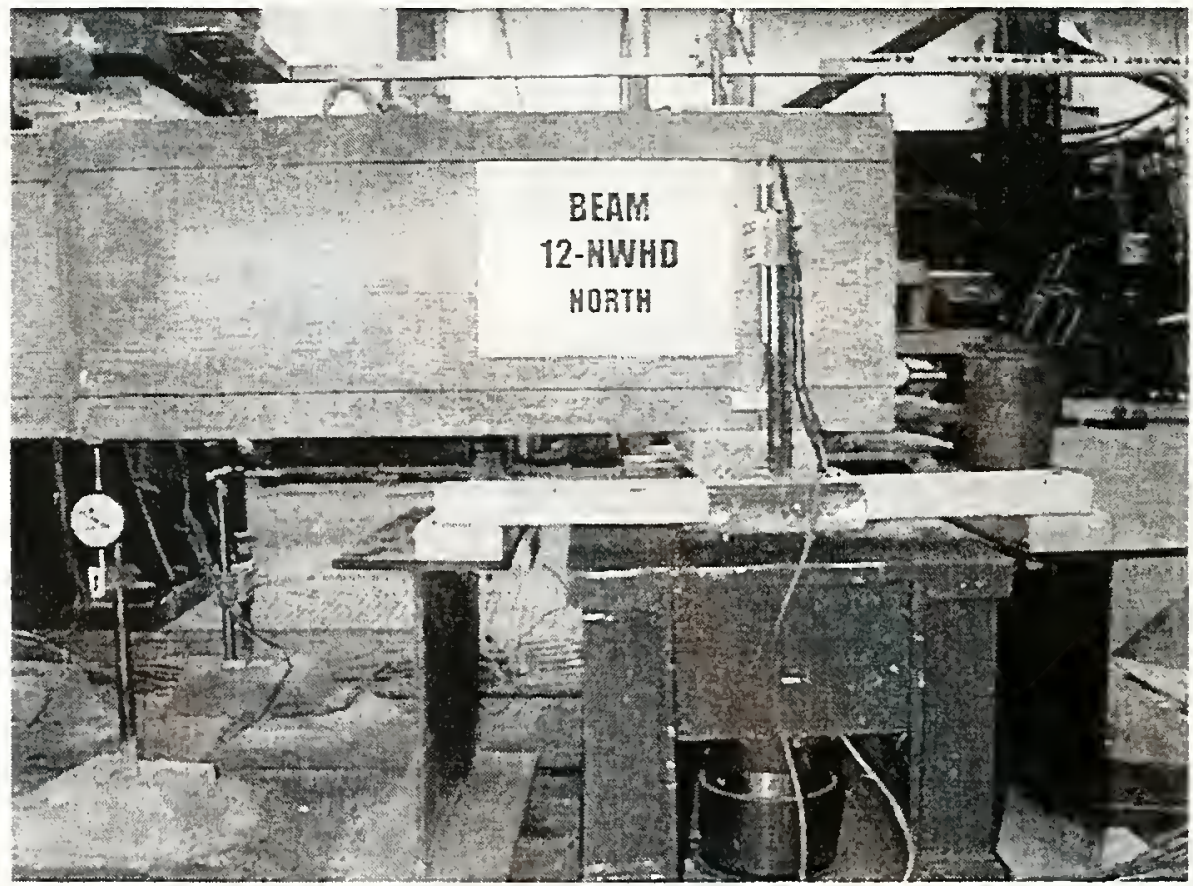

Figure 5.14 Support Beam and Load Cells at North End of Typical Reinforced Concrete Specimen (East Face) 


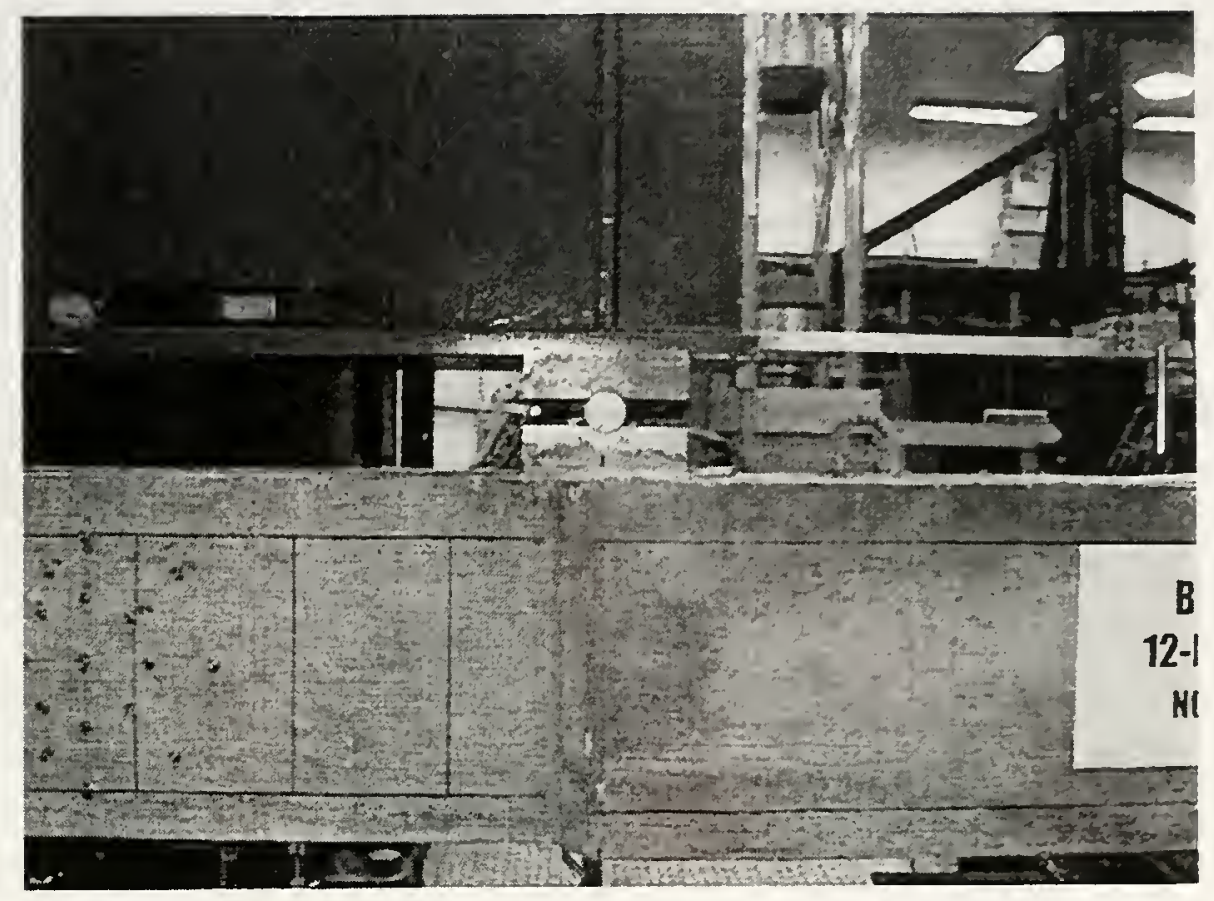

Figure 5.15 Loading Point at Maximum Positive Moment Section of Typical Reinforced Concrete Specimen (East Face) 

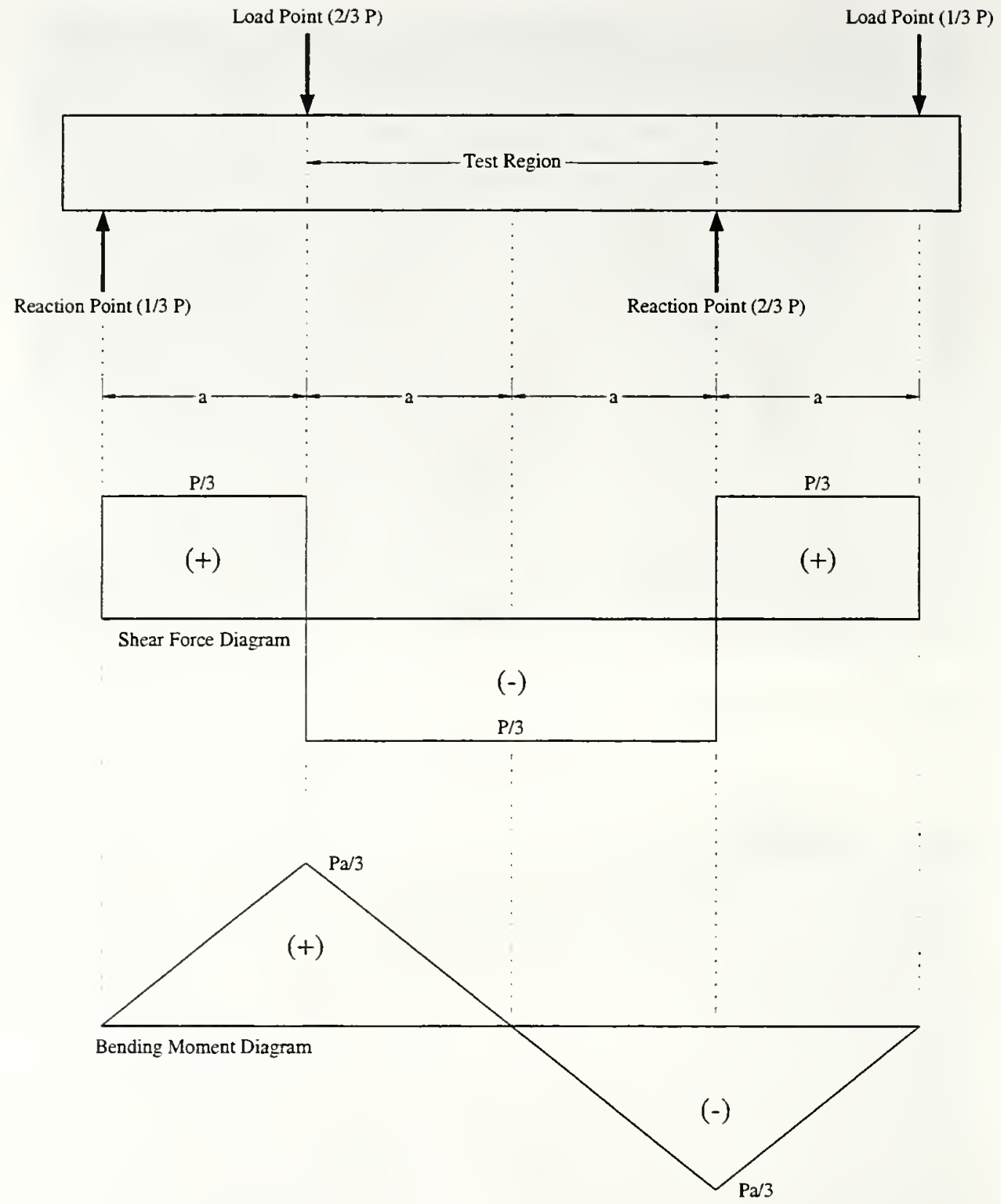

Figure 5.16 Shear Force and Bending Moment Diagrams for Reinforced Concrete Specimens 

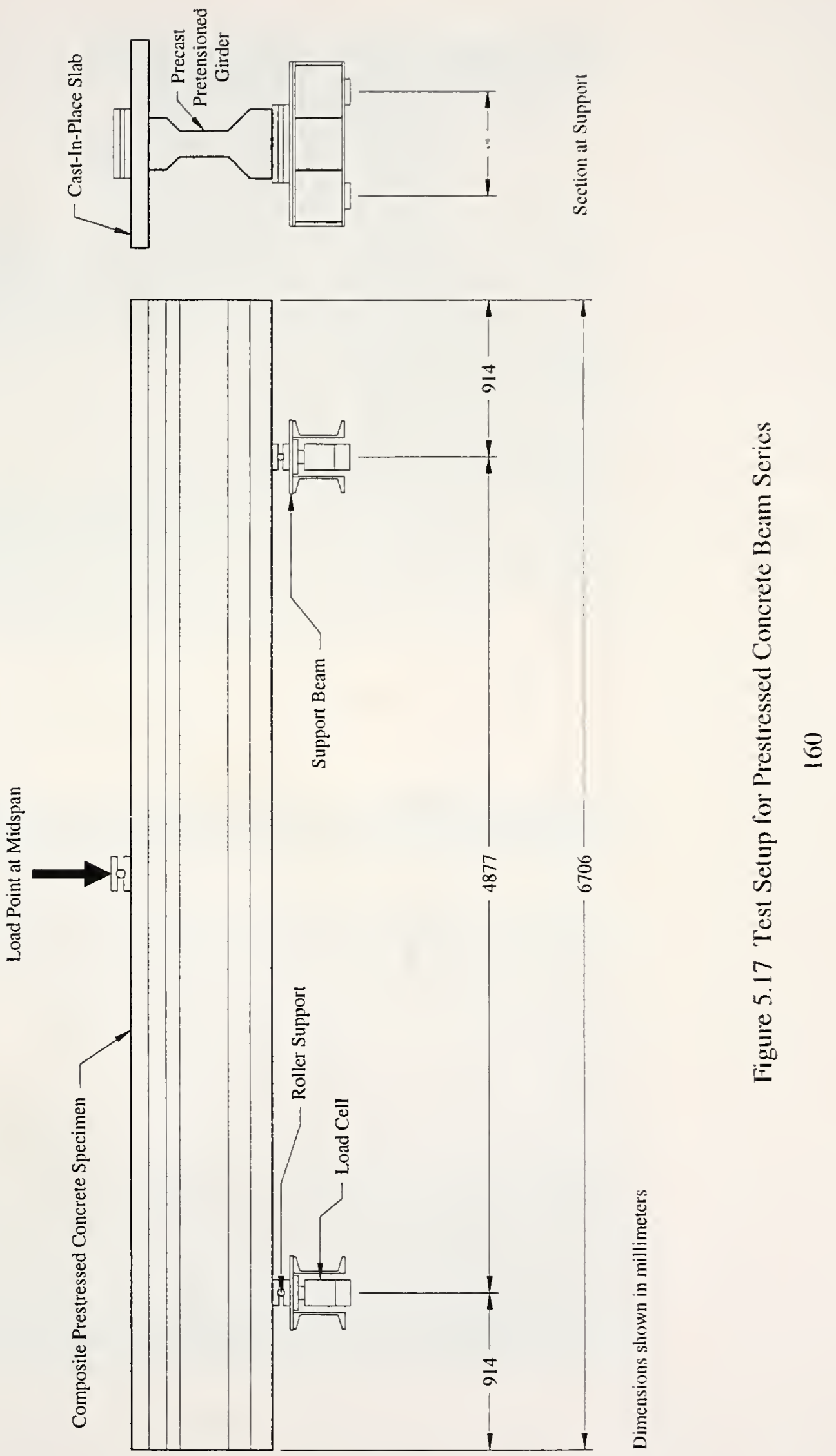


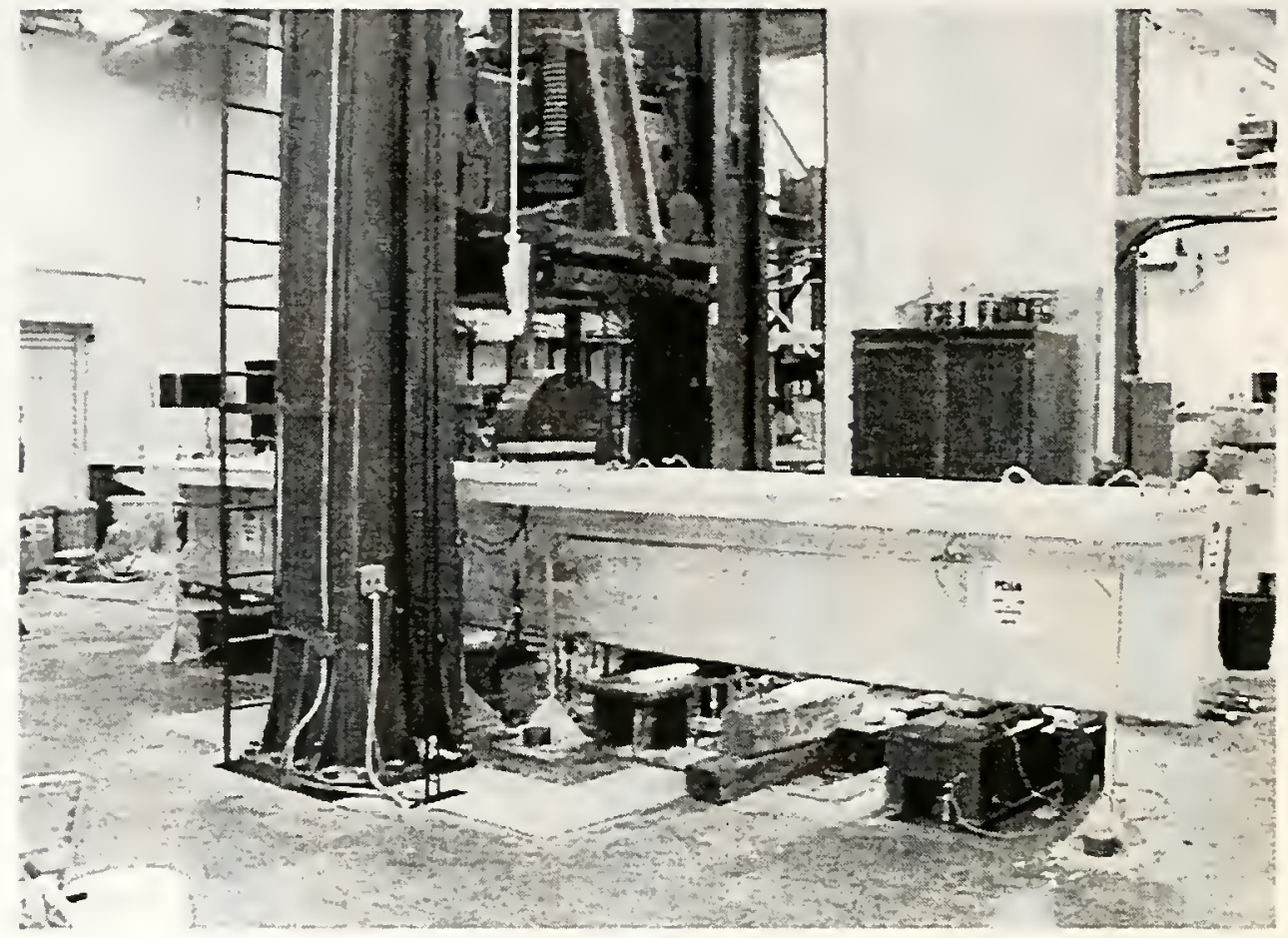

Figure 5.18 West Side of Prestressed Concrete Series Setup

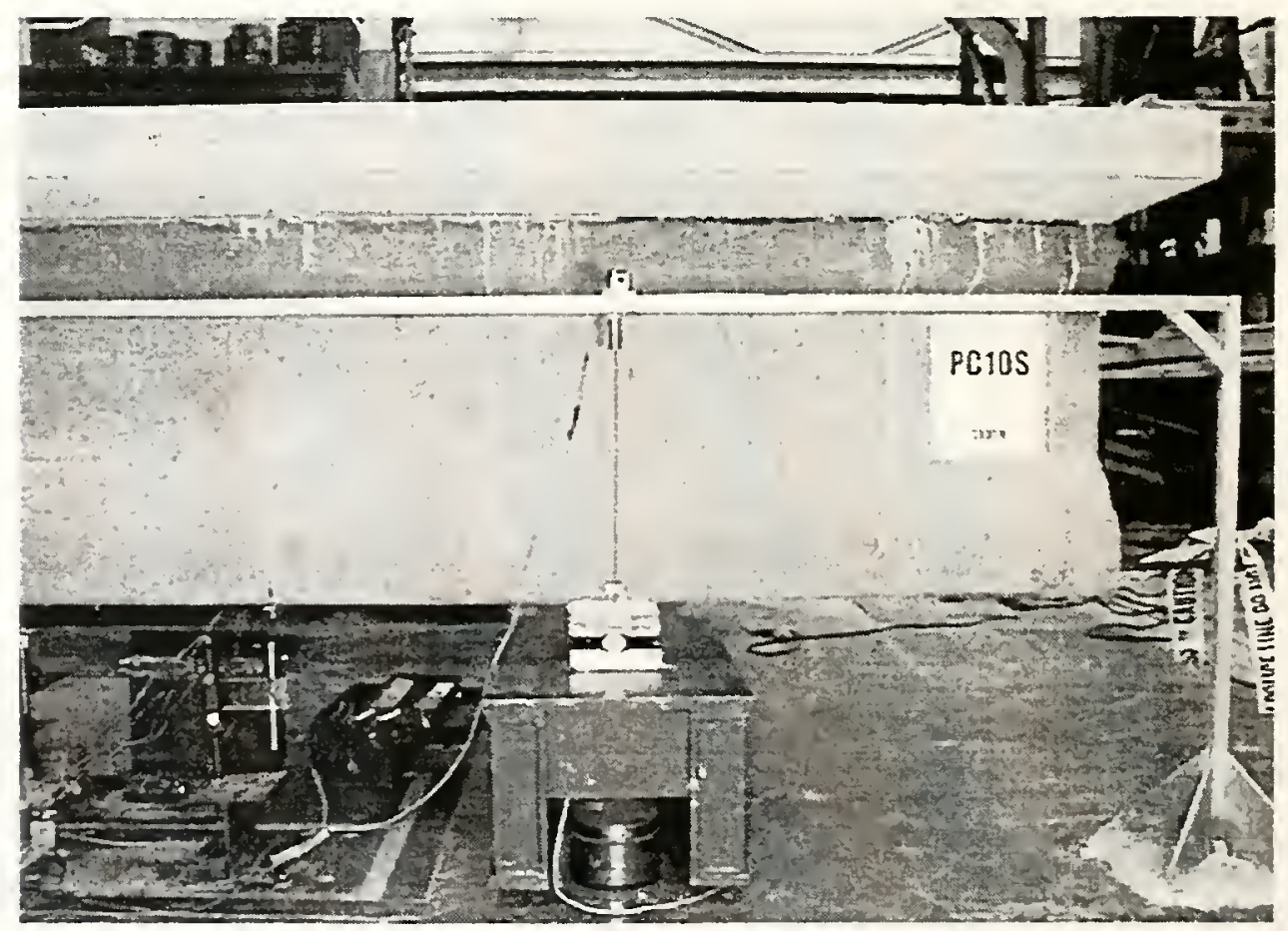

Figure 5.19 Support Beam and LVDT at South End of Prestressed Concrete Specimen 

